\title{
Evaluation of Seismic Behaviour of Hollow Fibre-Reinforced Polymer (FRP) Piles using Shake Table Testing
}

\author{
by
}

Mohammadamin Hosseini

A thesis submitted to the Faculty of Graduate and Postdoctoral Affairs in partial fulfilment of the requirements for the degree of

Doctor of Philosophy

in

Civil Engineering

Carleton University

Ottawa, Ontario

(C) 2021, Mohammadamin Hosseini 


\section{Abstract}

This study aimed at investigating the seismic performance of hollow FRP piles compared to traditional piles in fine sand and soft clay deposits using shaking table tests. A laminar shear box with a dimension of $1.0 \mathrm{~m} \times 1.0 \mathrm{~m}$ and a depth of $1.0 \mathrm{~m}$ was employed to contain the soil medium and allow the soil to respond in the same fashion as the free field. Two types of composite group piles $(2 \times 2)$ made of Carbon Fibre-Reinforced Polymer (CFRP) and Glass Fibre-Reinforced Polymer (GFRP) along with a series of Aluminium piles were manufactured and embedded as frictional and end-bearing piles within the soil. Several monitoring instruments were used to observe the soil-pile response under variety of ground motions adopted from the 2010 Val-desBois Earthquake in Canada and the 1995 Kobe Earthquake.

Seismic response of the foundation was strongly dependent on the stiffness provided by the soil, which was a function of the degree of softening and intensity of shaking. In both soil profiles, the foundation motion of model piles was higher than those of the free field under various intensity of input motions $(0.02-0.16 \mathrm{~g})$. This was attributed to strong interaction between the soil and the foundation, and flexural stiffness of the model piles. However, the frequency content of the amplified motions appeared to be consistent for all model piles.

In cohesionless soils, the aluminium pile cap experienced significantly higher acceleration and stronger rocking response compared to the FRP piles. Among the FRP piles, hollow GFRP pile was shown to be an appropriate alternative and replacement for frictional traditional piles. The shaking tests in fine sand also revealed that generation of pore water pressure has essentially affected the seismic response of FRP piles as well as traditional aluminium piles. This behaviour could be related to possible rearrangement of sand particles during shaking and the contraction tendency that resulted in a sudden increase of pore water pressure, leading to upsurge in density 
and sand densification. This could have induced larger kinematic forces as observed with increase in intensity of shaking.

In cohesive soils, soft clay deposits were shown to particularly amplify seismic input motions, though surface motions were attenuated during very strong shakings due to soil nonlinearity and stiffness degradation. The surrounding soil has also evidently affected the response of FRP piles and the divergence of the pile behaviour throughout the shaft was attributed to pile material characteristics (e.g., flexural stiffness) and significant kinematic interaction between the model piles and soft soil. CFRP and GFRP piles exhibited lower acceleration values and, hence, larger flexibility to deflect. Alternatively, aluminium piles have reflected significant kinematic forces which further underscores the importance of pile ductility and hence the kinematic pile-soil interaction on seismic response of pile foundations in seismic prone areas. 
Dedicated to my dear parents and my brothers....... 


\section{Acknowledgements}

First and foremost, I would like to express my respect and gratitude to my supervisor, Professor Mohammad Rayhani, who has been a tremendous teacher, and mentor throughout my graduate studies at Carleton University. I will be forever grateful for his constant support, guidance, motivation, and patience throughout this work. I would like to thank my thesis examination committee Professor Charles-Darwin Annan from Laval University, Professor Siva Sivathayalan and Professor Dariush Motazedian from Carleton University and Professor Mamadou Fall from University of Ottawa for taking the time reviewing and providing insightful comments. Moreover, I would like to thank all my friends, and colleagues for their understating and support. I would like to extend my sincerest thanks to the staff in the Civil Engineering Laboratory at Carleton University including Stanley Conley, Jason Arnott, Pierre Trudel, and Kenneth Akhiwu. Last, but not least, I would like to extend a heartfelt thanks to my parents and my brothers for their unconditional love, encouragement, patience, and sacrifices throughout all these years, which enabled me to keep focus on my study. 


\section{Table of Contents}

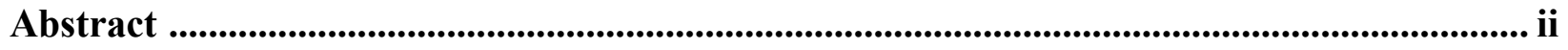

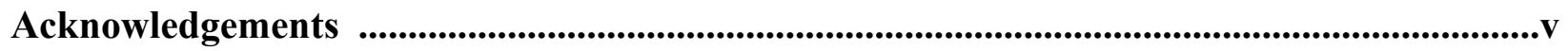

Table of Contents .......................................................................................................... vi

List of Figures ...........................................................................................................................................

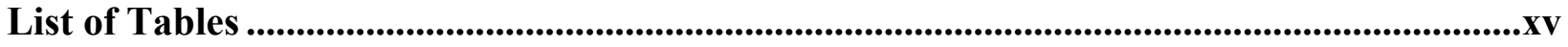

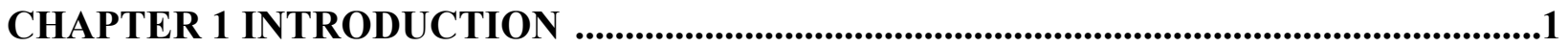

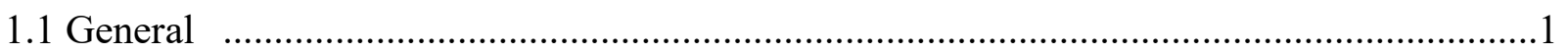

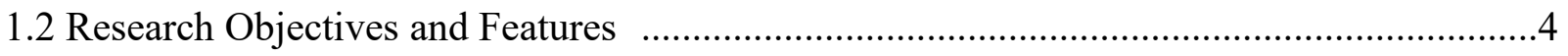

1.3 Organization of Dissertation .......................................................................................6

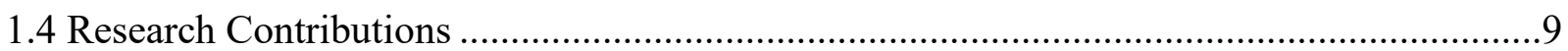

CHAPTER 2 LITERATURE REVIEW .............................................................................10

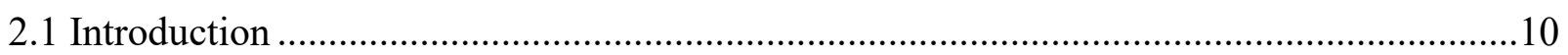

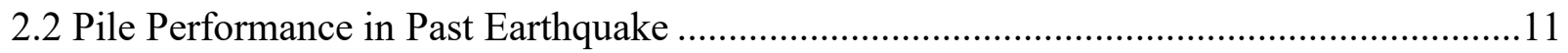

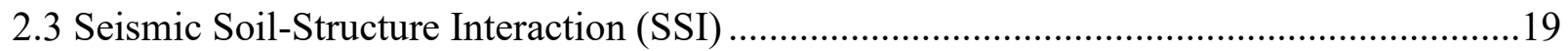

2.4 Effect of SSI on Foundation and Structural Response ......................................................23

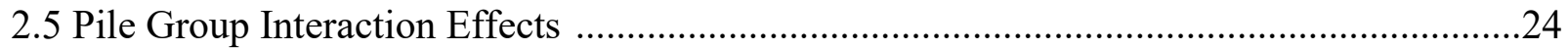

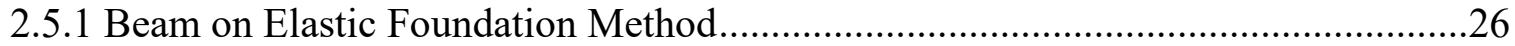

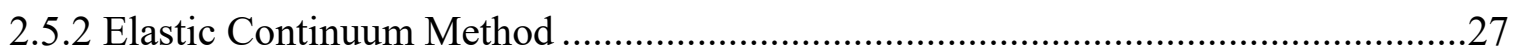

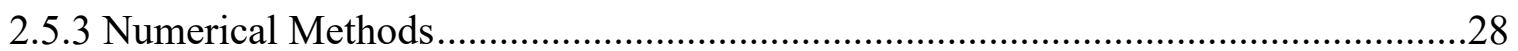

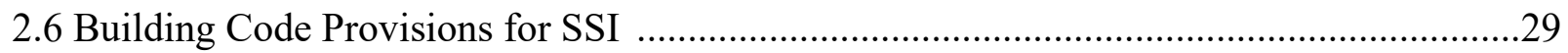

2.6.1 National Earthquake Hazards Reduction Program ....................................................30

2.6.2 American Society of Civil Engineers (ASCE 7-10) .....................................................

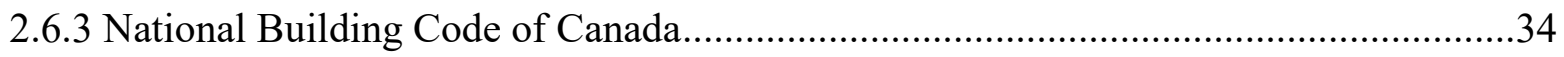

2.7 Mechanics of Composite Pile Materials …………….........................................................34

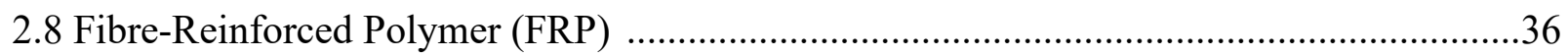

2.8.1 Glass Fibre-Reinforced Polymer (GFRP) ………..................................................

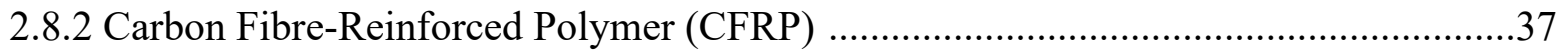

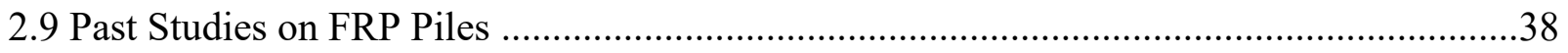

2.10 Previous Experimental Studies on Seismic Soil-Pile Interaction......................................42

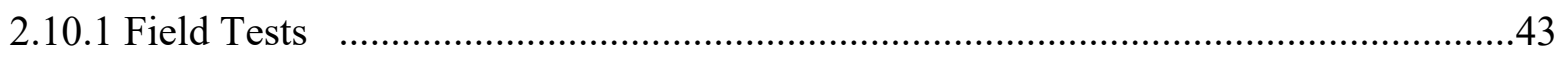

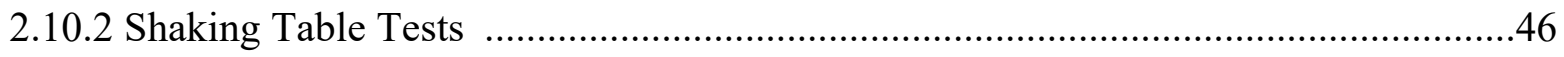


2.10.2.1 Earthquake Simulation using Shaking Table ……………….............................47

2.10.2.2 Classification of Shaking Table Models ........................................................49

2.10.2.3 Previous Shaking Table Tests on Pile Foundations ............................................54

2.11 Scaling Factors for Shaking Table Tests ...................................................................61

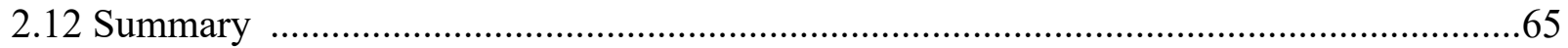

CHAPTER 3 EXPERIMENTAL PROGRAM …...................................................................67

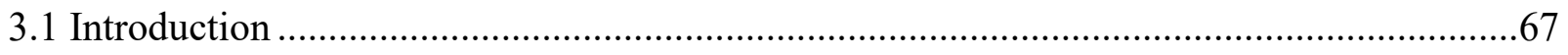

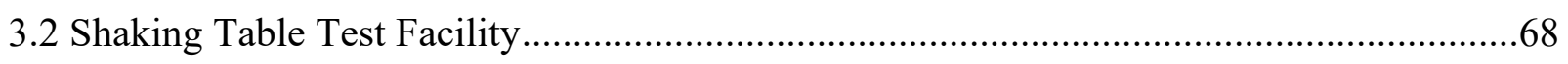

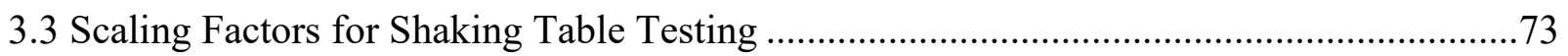

3.4 Model Pile Foundations ...........................................................................................73

3.4.1 Fabrication and Installation of Pile Groups ………………………………….....75

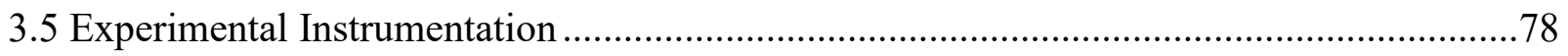

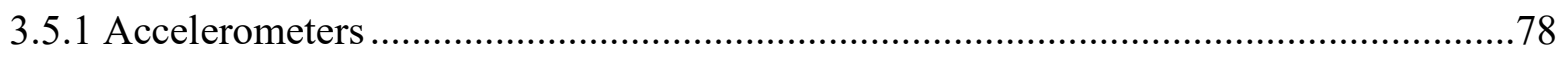

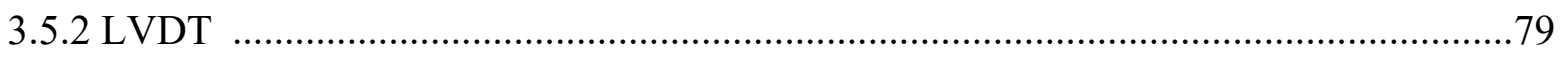

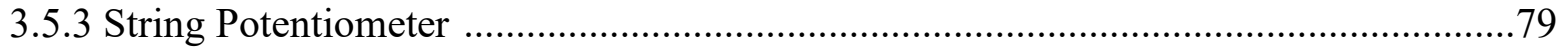

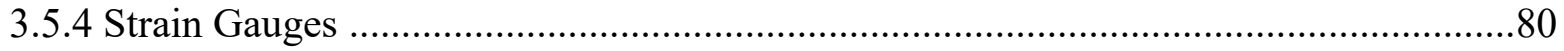

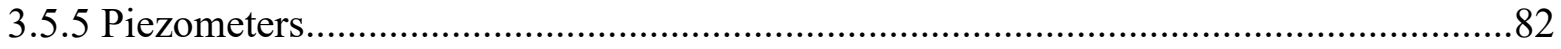

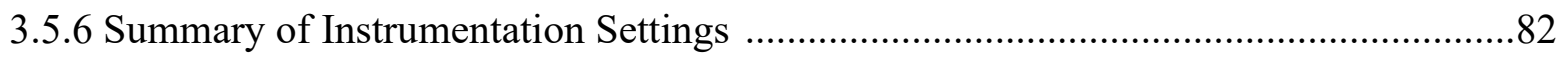

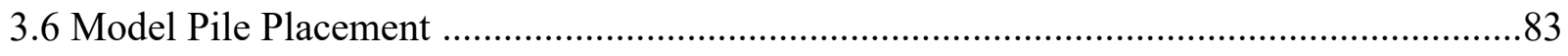

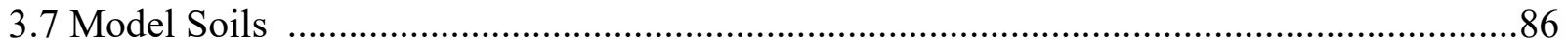

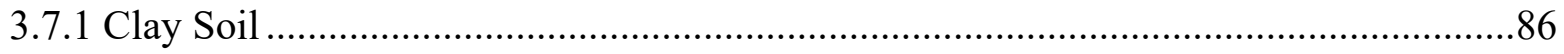

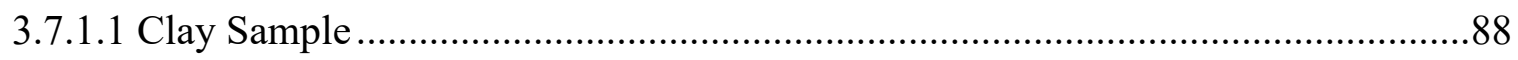

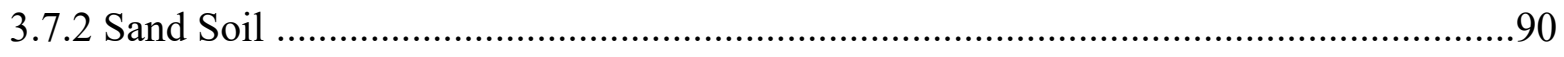

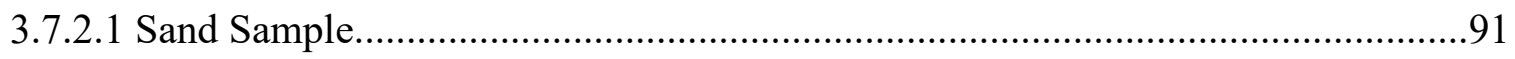

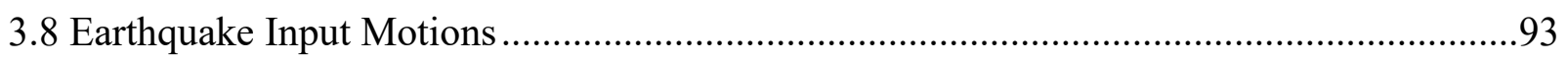

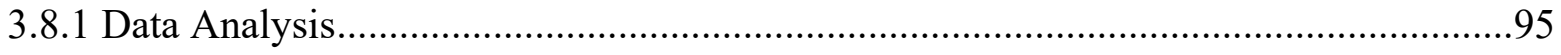

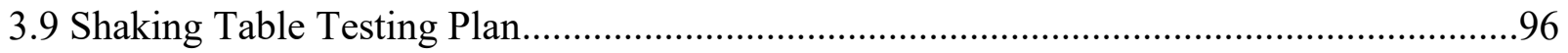

CHAPTER 4 Seismic Response of End-Bearing Fibre-Reinforced Polymer (FRP) Piles in Cohesionless Soils ..................................................................................................................98

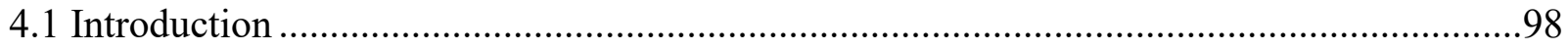

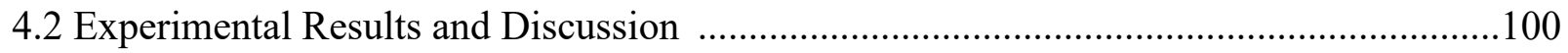

4.2.1 Acceleration Time Histories ……….....................................................................100 


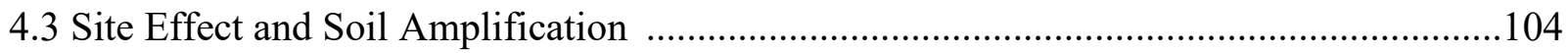

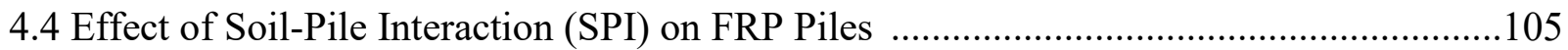

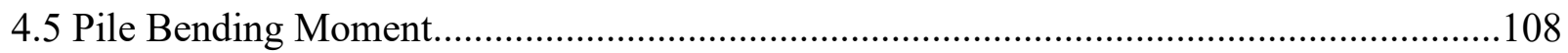

4.6 Impact of Liquefaction on Lateral Response of Pile Models .............................................110

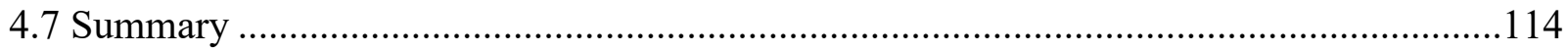

CHAPTER 5 Seismic Performance of Frictional FRP Piles in Sand.....................................116

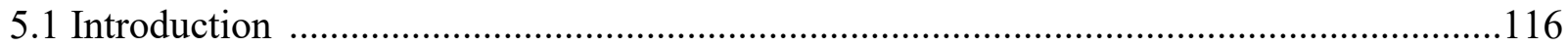

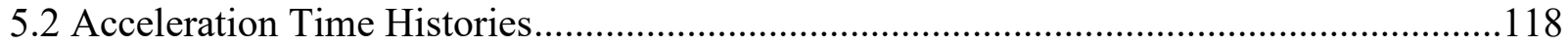

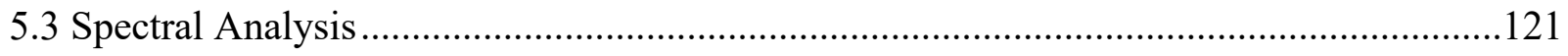

5.3.1 Seismic Site Response: Free Field Motion ……………………………….........121

5.3.2 Kinematic Soil-Pile Interaction (SPI) ………….................................................122

5.4 Pile Bending Moment …………………………..................................................126

5.5 Pore Water Pressure and Liquefaction Assessment ………………………………........130

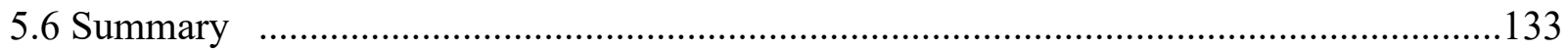

CHAPTER 6 Seismic Response of End-Bearing Fibre-Reinforced Polymer (FRP) Piles in

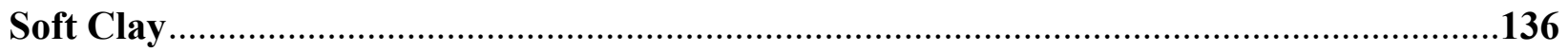

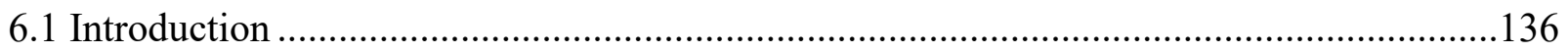

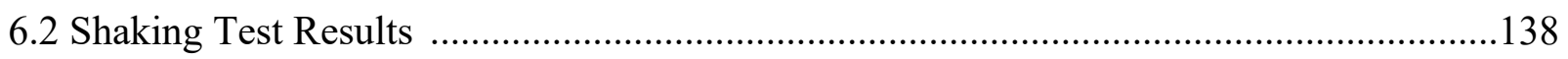

6.2.1 Acceleration Time Histories ............................................................................138

6.3 Spectral Analysis ......................................................................................................141

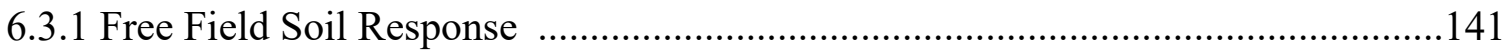

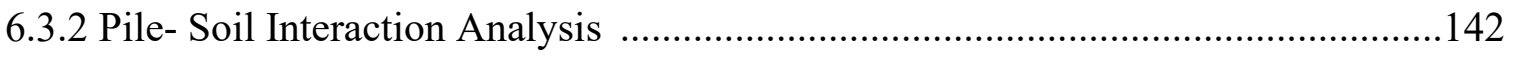

6.3.3 Impact of SPI on Pile Cap Motion..................................................................147

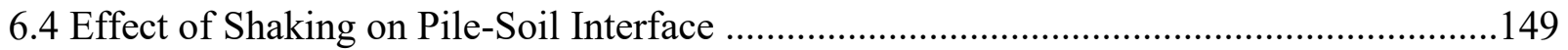

6.5 Bending Moment of Pile Shaft and Pile Cap Deflection ...................................................150

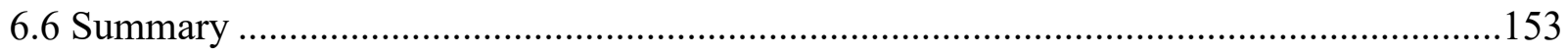

CHAPTER 7 Investigating Performance of Fibre-Reinforced Polymer (FRP) Model

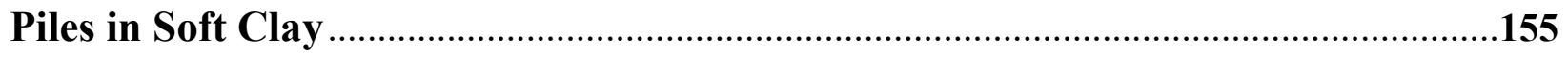

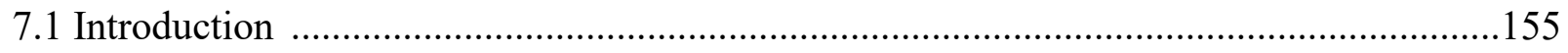

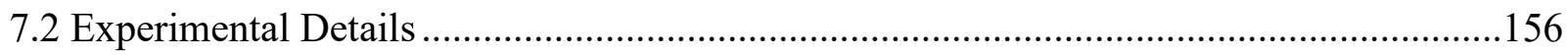

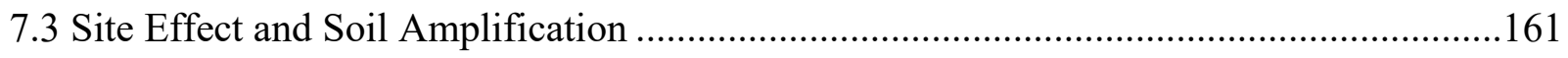

7.4 Spectral Acceleration on Model Piles .............................................................................162 
7.5 Effect of SPI on Frictional Pile Cap Response .............................................................164

7.6 Impact of Flexural Stiffness on Performance of Piles.......................................................165

7.7 Seismic Response of Frictional vs End-bearing Model Piles in Soft Clay .........................168

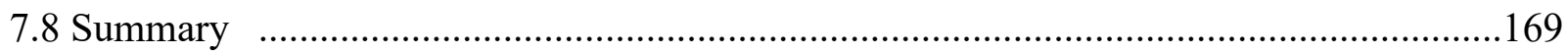

Chapter 8 Summary, Conclusions, and Recommendations ....................................................171

8.1. Research Summary and Findings .................................................................................171

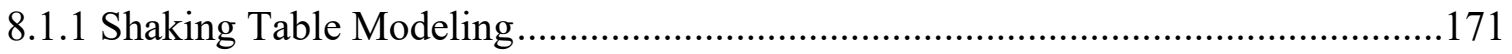

8.1.2 Description of Model Piles .............................................................................172

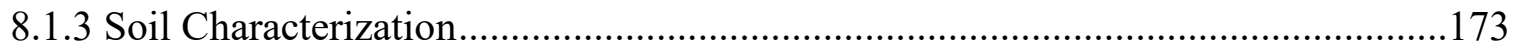

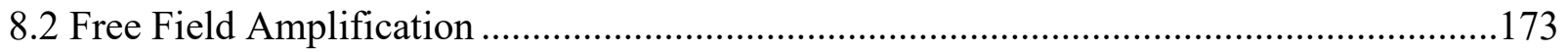

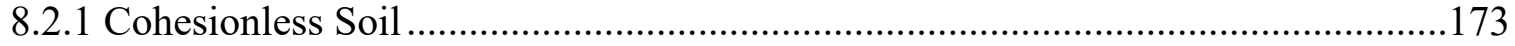

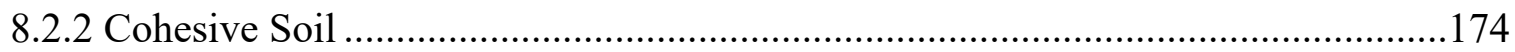

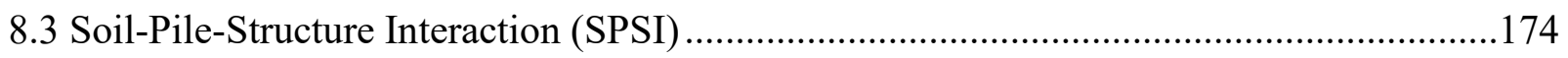

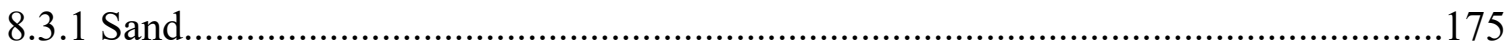

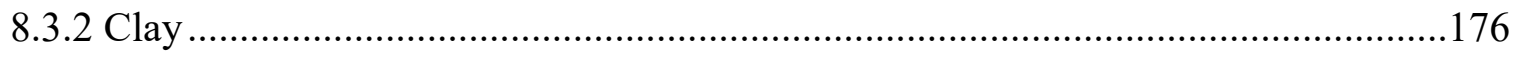

8.4 Conclusions and Recommendations............................................................................ 178

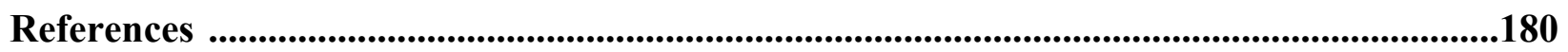

Appendices .............................................................................................................................................191 


\section{List of Figures}

\section{Chapter 1}

Figure 1.1: (a) Corroded steel pile (b) deteriorated concrete pile (c) deteriorated timber pile (Iskander and Hassan 1998)

Figure 1.2: (a) Replacement of timber piles by FRP pipe piles in Liberty Statue Dock by Creative

Pultrusion company (b) Installation of FRP pipe piles for pedestrian bridge on shore ......4

\section{Chapter 2}

Figure 2.1: Failure of pile supported pier of the Salinas (after Wood, 1908) ................................12

Figure 2.2: Timber Pile failure at Moss Landing (after Wood, 1908) ………….......................12

Figure 2.3: (a) Bridge 605 failure due to liquefaction in the Alaskan Earthquake, 1964 (Ross et al., 1973); (b) Timber piles penetrated through the concrete deck of Bridge 629 in the Alaskan

Earthquake,1964 (Ross, et al., 1973) .13

Figure 2.4: Ten story building supported by pile foundations in soft soils deposits during the 1985 Mexico City Earthquake: a) Site conditions; b) Overturned structure (after Mendoza and Auvinet, 1988)

Figure 2.5: (a) Development of gap around the pile; (b) Flexural failure of pile supporting cross bridge during Loma Prieta earthquake (after Seed et al., 1990)

Figure 2.6: Bearing failure of foundation in Niigata Earthquake, 1964

(United States Geological Survey)

Figure 2.7: Hanshin Highway Bridge failure during the 1995 Kobe Earthquake

(after Gazetas \& Mylonakis, 1998)

Figure 2.8: Separation of SSPSI Analysis into Kinematic and Inertial Interaction Components (after Waas and Hartmann, 1981)

Figure 2.9: Results of lateral response of steel pile group: a) Cyclic degradation of resistance; b) Load distribution within group in each row (after Brown et al., 1987). .25

Figure 2.10: Soil-structure interaction model including a single degree of freedom (SDOF) structure and discrete system to signify the supporting soil (after Wolf, 1985)

Figure 2.11: Geometric profile of acknowledged FRP piles in industry. (a) steel pipe core, (b) structurally reinforced plastic, (c) concrete-filled FRP, (d) reinforced plastic piles, (f) hollow FRP piles and (g) FRP sheet piles (Guades et al., 2012)

Figure 2.12: (a) Unidirectional lamina; (b) multidirectional laminate (Fam, 2000) ......................37

Figure 2.13: Fabrication and handling of precast composite piles (Fam et al, 2003) ...................41

Figure 2.14: Two-column pier shake table setup (Zaghi et al., 2012) ..........................................42 
Figure 2.15: Lateral pile load test setup for combined lateral and axial compressive loads (ASTM

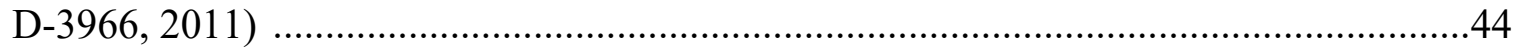

Figure 2.16: Wave propagation in piles during dynamic pile load test ( $\mathrm{Ng}$ et al., 2011) ...........45

Figure 2.17: Comparison of (a) the free field ground motion, and (b) the simulated motion employing rigid soil container on shaking table ......................................................51

Figure 2.18: Flexible cylindrical soil container (Meymand, 1998) .......................................52

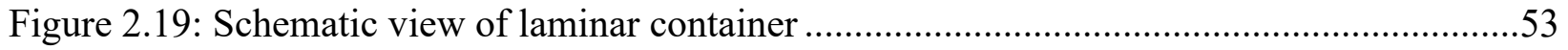

Figure 2.20: Model pile installation in shake table test (Meymand, 1998) ...............................56

Figure 2.21: Example of a shaking table tests for the multi-story structure with

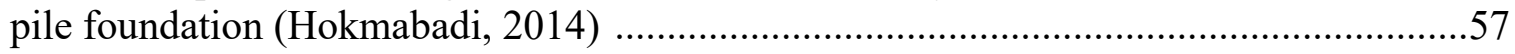

Figure 2.22: Schematic diagram of the shaking test setup (Taha, 2014) ...................................58

Figure 2.23: (a) Model group-pile foundation and superstructure. (b) Sand dropping device lay on an up-down movable frame Bao et al., (2012) ..........................................................59

Figure 2.24: Experimental setup for performing shaking table tests ......................................60

\section{Chapter 3}

Figure 3.1 (a): Installed shake table by manufacturer; (b): Side view schematic drawing of shake table

Figure 3.2: (a) Schematic drawings of laminar box container assembly (b) Final stage of assembled shake table with laminar container in Civil Engineering Lab, Carleton University ..........71

Figure 3.3: Latex membrane assembly for waterproofing the laminar container.......................72

Figure 3.4: (a) Shake table data acquisition system (b) shaker control software (i.e., SS10K) ....73

Figure 3.5: Glass FRP model pile (top), Carbon FRP pile (middle), and aluminium model

pile (bottom)

Figure 3.6: A view of GFRP group pile and aluminium group pile placed in shaking container, and a schematic drawing of pile cap dimensions......

Figure 3.7: Four-point bending flexural test using UTS, before and after failure of CFRP member.

Figure 3.8: (a) PCB Accelerometer used in this research; (b) ICP signal conditioner system (c) sample of attached accelerometer to pile shaft

Figure 3.9: String Potentiometer used to measure displacement of laminar soil container...... .81

Figure 3.10: (a) Glued bending strain gauge on pile shaft; (b) cDAQ module for strain gauge sensors; (c) location of attached strain gauges along the pile shaft 
Figure 3.11: Standard vibrating wire piezometer .82

Figure 3.12: Front and side view of instruments positions inside the laminar container .84

Figure 3.13: (a) \&(b) Placement of model piles in synthetic clay; (c) \&(d) placement of model piles in sand .85

Figure 3.14: Extracted undisturbed soft clay for shaking table tests at Carleton University..........86

Figure 3.15: Synthetic clay mixed in buckets and prepared for model soil...................................89

Figure 3.16: Preparation process for transferring synthetic clay inside laminar container .89

Figure 3.17: Various components of the shaking table models in synthetic clay medium with the group pile foundation adopted in this study .90

Figure 3.18: Particle size distribution of the test soil. .92

Figure 3.19: (a) Sand pluviation technique (left); and completion of sand pluviation and initiation for first excitation (right) (b)Various components of the shaking table tests in liquefiable soil

Figure 3.20: Scaled shaking events adopted in the shaking table experimental tests: acceleration time histories and response spectra at 5\% damping for 2010 Ottawa earthquake at Val-desBois in Province of Quebec .95

Figure 3.21: Scaled shaking events adopted in the shaking table experimental tests: three acceleration time histories at different intensity, and response spectra at 5\% damping for Kobe Earthquake, at Takatori recording station .95

\section{Chapter 4}

Figure 4.1: Schematic drawing of instruments used in model test; Accelerometer $\left(A_{n}\right)$; LVDT, Strain gauges and PWP sensors 102

Figure 4.2: Acceleration time histories for the CFRP and Free Field at $200 \mathrm{~mm}$ depth during the Kobe 20 shaking event

Figure 4.3: Response spectra for free field during the $\mathrm{K} 5$ and $\mathrm{K} 20$ excitations 104

Figure 4.4: Ratio of response spectra (RRS) of pile models along the shaft during O100 and K20 shaking event 106

Figure 4.5: Pile cap reponse spectra at $\mathrm{O} 100$ and K20 shaking events for all model piles 108

Figure 4.6: Recorded bending moment distribution along the piles at $\mathrm{O} 100$ and $\mathrm{K} 20$ 109

Figure 4.7: Pile head/cap deflection at the K20 event 110 
Figure 4.8: Excess pore water pressure during the Kobe shaking event- T2.

Figure 4.9: (a) Rate of pore water pressure; (b) Soil settlement for all shaking events at $\mathrm{T} 1$ and $\mathrm{T} 2$.

Figure 4.10: Development of sand boiling during the eaqrthquake loading (left to right) 114

\section{Chapter 5}

Figure 5.1: Schematic drawing of instruments used in model test; Accelerometer $\left(\mathrm{A}_{\mathrm{n}}\right)$; LVDT, Strain gauges and PWP sensors 119

Figure 5.2: Sample acceleration time histories for the CFRP pile and free field response at base \& surface during the Kobe 20 excitation

Figure 5.3: Response spectra for free field motions during the O100 and K20 shaking events..122

Figure 5.4: Ratio of response spectra (RRS) for all pile models during O100 and K20 shaking events

Figure 5.5: Pile cap reponse spectra at $\mathrm{O} 100$ and K20 shaking events for all model piles .........126

Figure 5.6: Recorded bending moment distribution along the piles at O200, and $\mathrm{K} 20$..............128

Figure 5.7: Pile head/cap deflection at the K20 event

Figure 5.8: Laminar displacement at the K20 event. 17Figure 5.9: Rate of pore water pressure for all shaking events of $\mathrm{T} 3$ and $\mathrm{T} 4$

Figure 5.9: Rate of Pore water pressure and Soil Settlement for all shaking events 132

Figure 5.10: Settlement of soil deposit after each shaking event

Figure 5.11: Development of sand boiling during the eaqrthquake loading (left to right)

\section{Chapter 6}

Figure 6.1: Shaking table models' configuration in laminar box

Figure 6.2: Acceleration time histories free field and CFRP at K20 shaking

Figure 6.3: Response spectra for free field motions during the O100, K5 and K20 shaking events

Figure 6.4: Pile/soil response spectral ratio (RRS) for all models during O100, K5 and K20 shaking events .144

Figure 6.5: Spectral acceleration of model piles at O100, K5, and K20 excitations 146 
Figure 6.6: Pile cap reponse spectra at K5 and K20 shaking events for all model piles .148

Figure 6.7: Development of gapping around model piles; Aluminium (left) and CFRP (right) 150

Figure 6.8: Recorded bending moment distribution along the piles at $\mathrm{K} 5$ and $\mathrm{K} 20$ 152

Figure 6.9: Pile head/cap deflection at K20.

\section{Chapter 7}

Figure 7.1: Instruments configuration in laminar container

Figure 7.2: Acceleration time histories free field and GFRP at K20 shaking 158

Figure 7.3: Recorded maximum acceleration of free field and model piles at different seismic loading (FF, free field; AL, aluminium pile; CFRP, carbon FRP, GFRP, glass FRP) ...160

Figure 7.4: Free field spectral acceleration during the $\mathrm{O} 200$ and K20 shaking events

Figure 7.5: Spectral acceleration of model pile at $\mathrm{O} 200$ and $\mathrm{K} 20$ excitations .163

Figure 7.6: Pile/soil ratio of response (RRS) all models during O200 and K20 shaking event ..164

Figure 7.7: Pile/soil response spectral ratio (RRS) for all model piles during O200 and K20

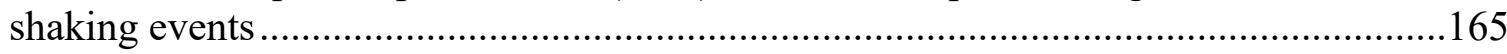

Figure 7.8: Pile cap reponse spectra at $\mathrm{O} 200$ and K20 shaking events for all model piles ........167

Figure 7.9: Recorded bending moment distribution along the piles at $\mathrm{O} 200$ and $\mathrm{K} 20$

Figure 7.10: Pile cap reponse spectra at O200 (left) and K20 (right) shaking events for frictional and end-bearing FRP model piles 168 


\section{List of Tables}

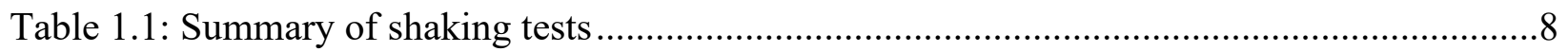

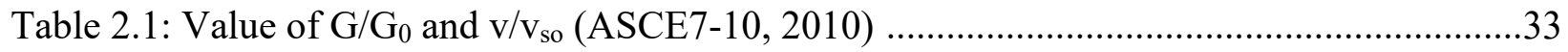

Table 2.2: Summary of past shaking table tests using various types of soil containers .................61

Table 2.3: Scaling relations for shaking table in terms of geometric scaling factor $(\lambda)$ - (Iai, 1989)

Table 3.1: Key properties of the SS10K-05 laminar shaker ......................................................70

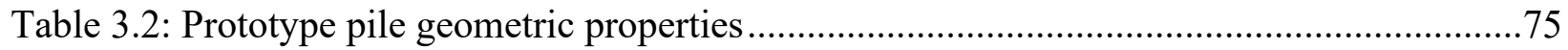

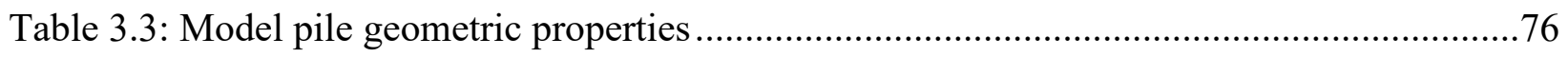

Table 4.1: Summarized peak accelerations at free field, pile cap and along the pile shafts for all

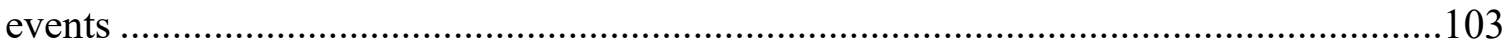

Table 5.1: Summarized peak accelerations at free field, pile cap and along the pile shafts for all

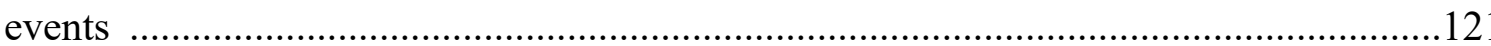

Table 6.1: Summarized peak accelerations at free field, pile cap and along the pile shafts for all

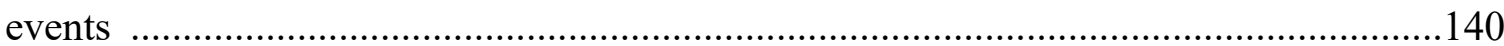

Table 7.1: Recorded Peak accelerations for different excitations in all experimental events .....158 


\section{CHAPTER 1: INTRODUCTION}

\subsection{General}

Pile foundations are widely used to support high load structures in compressible and weak ground strata throughout the world. Piles are relatively long elements with generally slender geometry and structural foundations that transfer structural loads to firmer soil layers. The response of structures resting on stiff soil or rock may be significantly simpler to evaluate since the response can be close to free-field motion. However, this response can vary if structures are supported by a liquefiable soil and/or soft soil deposits. In such site conditions, the imposed motion to the structure diverges from the free-field motion as a result of the presence of stiffer structure and foundation systems. Moreover, dynamic response of the structure can lead to deformation of the supporting liquefiable and/or soft soil deposits. Several researchers studied seismic response of traditional pile foundations (i.e., concrete, steel, aluminium, etc.) and impact of the soil-pile interaction by conducting field and laboratory experiments (e.g., Hassini and Wood, 1989; Ohtomo, 1996; Tao et al., 1998; Wang et al., 1998; Fam, 2000; Knappett et al., 2006; Moss et al., 2010; Motamed et al., 2013; Yang et al., 2018).

Nevertheless, there is a great demand for piling poles, highway overhead sign structures and pedestrian bridges to be made of materials that are more durable in comparison to traditional construction materials and systems. The new products have to withstand aggressive corrosive environments such as the splash zone in case of marine piles, where they would be ultimately submerged and exposed (Stapleman, 1997). Similarly, highway overhead sign structures poles, and pedestrian bridge piers need to retain their integrity in cold regions where salt is used for deicing the roads. One promising innovative structural system is Fiber Reinforced Polymer (FRP) 
piles. The proposed system consists of hollow FRP round tubes which are very light materials and could provide the specific strength and stiffness depending on fabric type. A typical example of hollow FRP could also be concentric in an axisymmetric pattern which is suited for applications involving loads applied from various directions such as wind pressure or it could be eccentric to provide more reinforcement in the tension. While considerable number of research works was conducted to understand the load transfer and flexural response of various FRP piles by performing static tests (e.g., Fam, 2003; Hosseini and Rayhani, 2017), however, there are very limited studies on the seismic response of FRP piles in earthquake prone areas.

During earthquakes, seismic waves are generated in the bedrock, propagate through the soil layers and damage structures resting on the surface. The influence of local soil conditions and soil-pilestructure interaction (SPSI) on the intensity of foundation motion and earthquake damage has been recognized for many years. Local site conditions can profoundly influence the important characteristics of strong ground motion (i.e., amplitude, frequency content, and duration). Previous earthquakes such as the Kobe (1995), Loma Prieta (1989), and Mexico City (1985) earthquakes have clearly highlighted the role of local site conditions and soil-structure interaction in modifying and altering the characteristics of ground motions. Additionally, the construction industry is always looking for innovative pile design and/or materials that can perform reliably under seismic loads, and feasible to manufacture or construct. FRP materials have been introduced as a substitute material either as an actual pile foundation (variety of designs in terms of geometry) or a reinforcing material for restoration of pile foundations such as concrete/steel piles.

FRP piles have been used in several construction developments; one of these early projects was the Route 40 Bridge in Virginia using concrete filled FRP tubes as a substitute for precast piles. Another industrial project by Creative Pultrusion company was associated to replacement of 
timber piers of Statue of Liberty Dock in San Francisco; replacement of reinforced concrete piles in Long Beach pedestrian bridge and Legacy Dock piles by FRP pipe piling (Figure 1.2). These projects used FRP pipe piles because of material characteristics of the FRP pipe pile, in combination with the corrosion resistance characteristics, and the fact that the FRP pipe piles will not rot, rust, spall or corrode. FRP piles were found to be practical (e.g., lighter than all traditional pile materials), feasible (e.g., low maintenance and higher durability), environmentally friendly (i.e., no leach or preservatives left in environment), and uncomplicated manufacturing process (Juran and Komornik, 2006). However, lack of seismic investigation specifically on hollow FRP pile foundations in every application has been a concern in geotechnical practice, especially in liquefiable and soft grounds, and most of the past studies have recommended further investigation to understand the behaviour of FRP piles under seismic loadings. Additionally, model tests in geotechnical engineering provide the advantage of simulating complex systems under controlled conditions. This can offer advantages to verify the fundamental mechanism of SPSI and act as a calibration benchmark for numerical or analytical methods.

The present research aims to evaluate and quantify the response of hollow FRP group piles exposed to seismic loading compared to traditional group piles (e.g., aluminium) in liquefiable soils and soft clay considering soil-pile-structure interaction (SPSI), which is significantly important on the performance-based design of foundations and structures. Furthermore, various types of pile foundations (i.e., end-bearing and frictional piles) are considered to be investigated since the foundation type can alter the dynamic properties of the system (e.g., stiffness, damping and natural frequency). This investigation is carried out using 1-g shaking table tests. Several model piles made of Carbon and Glass FRP materials as well as aluminium piles were tested under 
various excitations, followed by a parametric study to evaluate the influence of seismic soil-pile interaction on the seismic response of hollow FRP piles in liquefiable soil and/or soft clay.
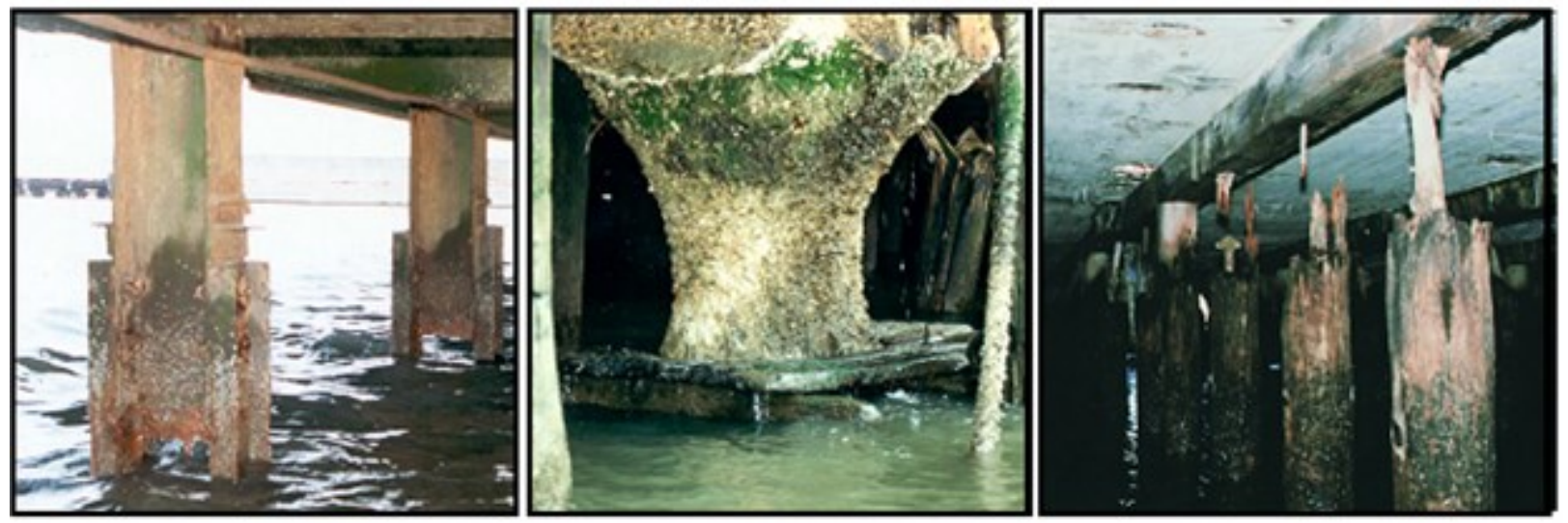

Figure 1.1: (a) Corroded steel pile (b) deteriorated concrete pile (c) deteriorated timber pile (Iskander and Hassan 1998)

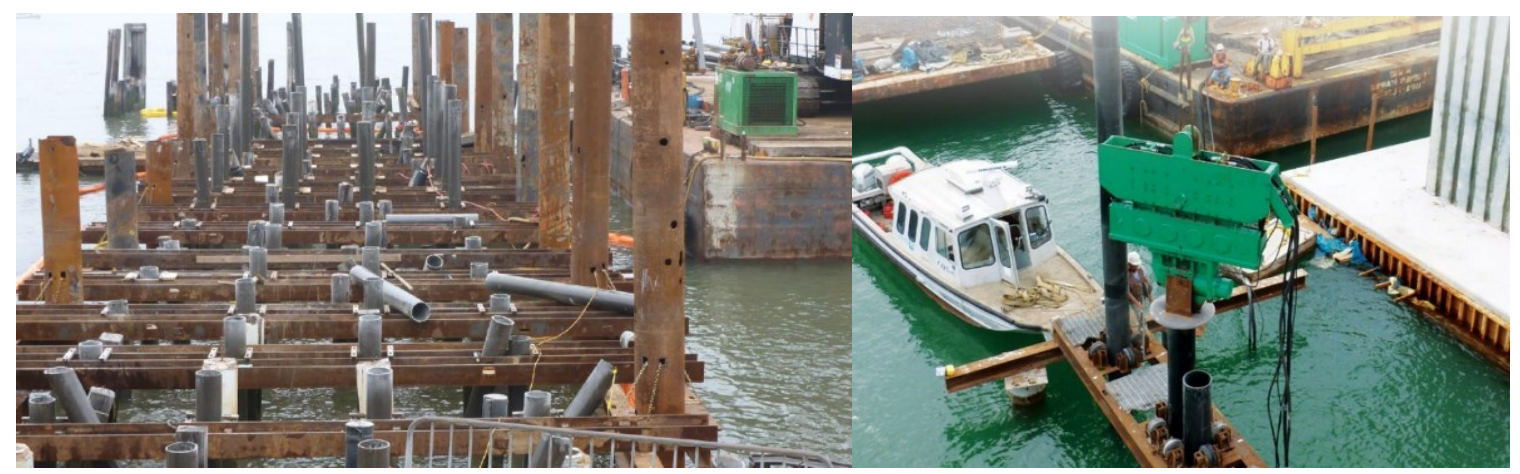

Figure 1.2: (a) Replacement of timber piles by FRP pipe piles in Liberty Statue Dock by Creative Pultrusion company (b) Installation of FRP pipe piles for pedestrian bridge on shore by Creative Pultrusion

\subsection{Research Objectives and Features}

This thesis describes the results of a study on the dynamic response of hollow FRP pile foundations in soft clay and liquefying sand during different excitations of real earthquake ground motions. The research consists of four components: (1) a series of shaking tests of FRP group and aluminium group piles in soft and liquefying soils performed (2) a critical investigation of physical modeling techniques and limitations; (3) pile response from recordings of pile acceleration along 
the shaft (i.e., at the toe and close to surface), pile bending moment, pile head response by recording acceleration and displacement at the cap, free field response profile from recorded accelerations in laminar container, and change in pore water pressure in liquefiable soil; and (4) evaluation of pile foundation type (i.e., end-bearing and frictional) and their kinematic interaction on their response.

Shaking table experiments were performed using several different pile foundations in terms of material (i.e., carbon FRP, glass FRP, and aluminium) and length (i.e., end-bearing and frictional pile models), different earthquake input motions (varying level of shaking, frequency content, and waveforms), and different uniform medium (i.e., liquefiable sand and soft clay).

The main objective of this study is to evaluate the seismic response of Fibre-reinforced polymer (FRP) piles (carbon and glass) by performing shaking table tests. The key objectives of this experimental investigations that evaluates different aspects considered in the study are:

a) Understand the significance of the soil-foundation interaction on the seismic response of hollow FRP piles.

b) Identify the effective parameters on the response of FRP piles and traditional piles over a range of base excitations o in two different uniform mediums (i.e., liquefiable soil and soft clay)

c) Evaluate the effects of site conditions on ground motion parameters and seismic response of FRP piles (carbon and glass) including pile group deflections, moment distributions of individual model pile per group.

d) Examine the impact of material characteristics such as flexibility on the kinematic interaction and additionally the influence of the hollow of FRP pile response on structural performance. 
e) Clarify the response of different foundation load transfers on the seismic response of pile cap and the superstructure in terms of rocking of cap/structure, and amplification due to lateral deformation.

f) Examine the response of various FRP materials (i.e., GFRP and CFRP) under seismic loading compared to traditional piles when exposed to wide range of seismic excitations.

\subsection{Organization of Dissertation}

A tremendous amount of data was collected in the course of this research. Data from a total of 8 series of shaking tests (i.e., each test undergoes 6 excitations) on three pile materials (Carbon FRP, Glass FRP, and aluminium) in arrangement of 2x2 pile-groups supported simple structure was obtained. This thesis consists of eight chapters, where Ch.4 to Ch.7 present shaking tests results conducted on various site condition and/or pile types in terms of material and length. These chapter are written as paper-based format, and minimal repetition was considered.

Chapter 1. Introduction - includes a brief discussion on the FRP piles and importance of understanding soil-pile-structure interaction. It also summarizes the research objectives, scope of work, and an organizational summary of the thesis.

Chapter 2. Background - provides an integrated literature review of the most relevant research works on existing experimental and analytical research on FRP piles. This includes a brief overview of published knowledge on soil-pile interaction that (1) revealed general features of behaviour for lateral loading of piles; (2) seismic loading of piles and experimental procedures of direct relevance to this present study for simulation of soil-pile interaction of traditional and FRP piles.

Chapter 3. Shaking Table Testing of Group Piles under Seismic Loading - this chapter presents the experimental setup and shaking table test procedures for model piles installed in 
granular and cohesive soils. Details of soil and model pile characteristics along with applied earthquake motions and instruments are also discussed here.

Chapter 4. Seismic Response of end-bearing group piles in cohesionless soil (CFRP, GFRP and aluminium) -this chapter is devoted to present laboratory shaking table test results of endbearing FRP group piles (i.e., carbon and glass) embedded in liquefiable soils. The impact of soilpile interaction on foundation input motion among hollow FRPs and aluminium tube piles under seismic loading are presented. This includes local site effect, change in pore water pressure during testing, and the seismic response of the pile system are also discussed.

Chapter 5. Shaking tests analysis of Frictional FRP group piles in cohesionless soils (CFRP, GFRP and aluminium) - this chapter presents the amplification of ground motion and the seismic behaviour of frictional piles under shaking tests in cohesionless soil. The seismic response of both FRP and traditional piles are analysed and discussed here.

Chapters 6 and 7 present results of shaking table tests on both frictional and end-bearing FRP and traditional piles in cohesive soils. Site effect and soil amplification, pile-soil interaction analysis, and influence of SPI on pile cap response are discussed in these two chapters.

Chapter 8 provides a summary of the research outcomes along with recommendations for potential future research. 
Table 1.1: Summary of shaking tests

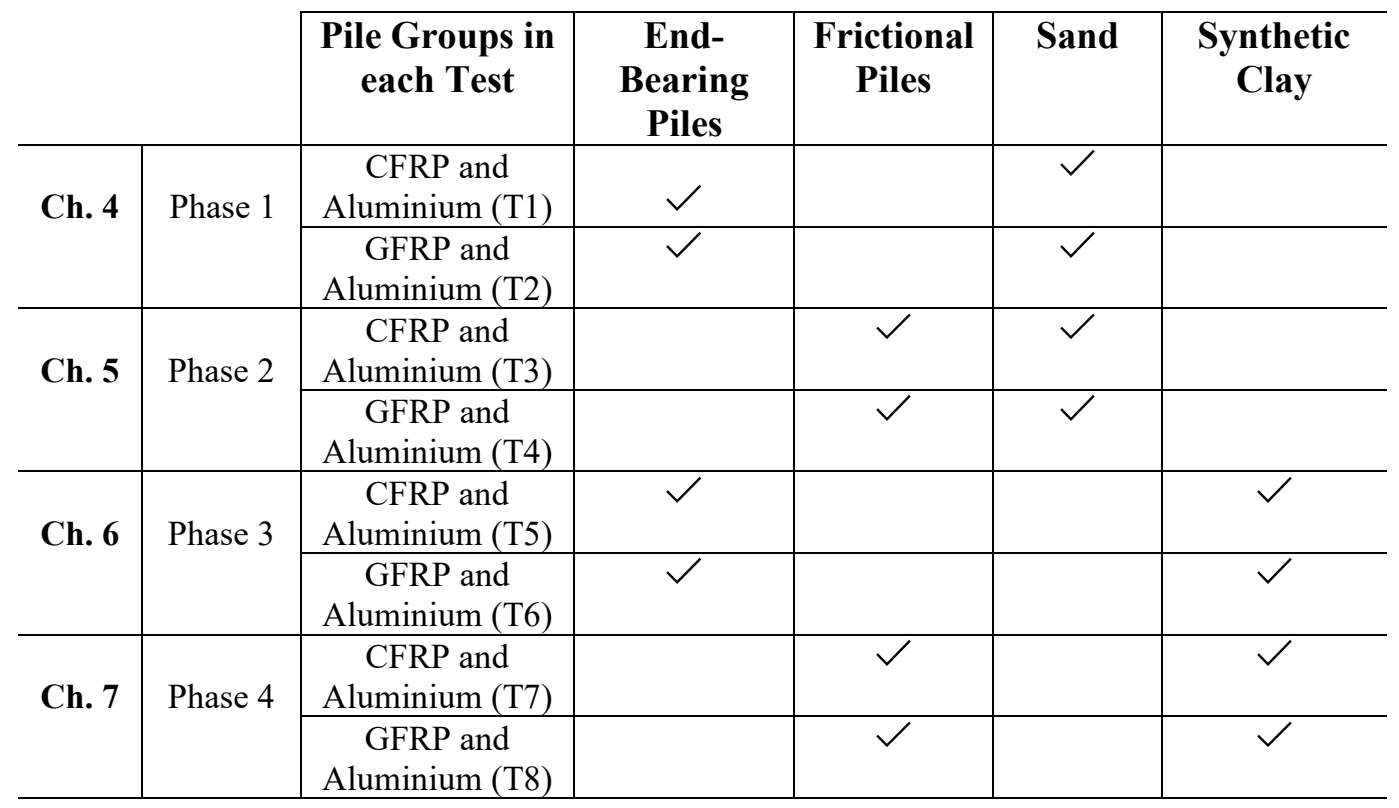




\subsection{Research Contribution}

- Hosseini M.A., Rayhani M. An Experimental investigation of the seismic behaviour of frictional hollow FRP group piles embedded in soft clay. Bulletin Earthquake Engineering, to be Submitted in July 2021.

- Hosseini M.A., Rayhani M. Seismic performance of end-bearing FRP piles in cohesionless soil. Journal of Geotechnical Research, to be Submitted in June 2021.

- Hosseini M.A., Rayhani M. Evaluation of seismic response of GFRP and CFRP group piles in soft clay. Canadian Geotechnical Journal, under review.

- Hosseini M.A., Rayhani M. Evaluation of seismic response of GFRP and CFRP group piles in cohesionless soils. Innovative Infrastructure Solutions, Nov. 2020 (under review).

- Hosseini M.A., Rayhani M. (2021). Shaking Table Tests of Model Hollow Fibre-reinforced Polymer Piles in Soft Clay. $74^{\text {th }}$ Canadian Geotechnical Conference. Niagara Falls, Canada.

- Hosseini M.A., Rayhani M. (2019). Seismic Performance of hollow Fibre-Reinforced Polymer (FRP) piles in cohesionless soils. $72^{\text {nd }}$ Canadian Geotechnical Conference. St. Johns, Canada 


\section{CHAPTER 2: LITERATURE REVIEW}

\subsection{Introduction}

Pile foundations are widely used to support high load structures in compressible and weak ground strata throughout the world. Thus, the main function of piles is to transfer loads from the structure further down to more competent soil/rock layer. Additionally, pile foundations are designed to provide a certain level of lateral resistance while sustaining the lateral deformation within limits required by the structural engineer. Piles are traditionally made of concrete, steel and timber; however, there is a great demand to explore and manufacture innovative piles using materials that can be more durable compared to traditional materials especially in harsh environments. From 1987, several common types of composite piles have been developed including reinforced plastic piles, concrete filled Fibre-Reinforced Polymer (FRP) piles, fiberglass pultruded piles, fiberglass reinforced plastic piles, hollow FRP piles and FRP sheet piles (Guades et al., 2012). Although, FRP materials may cause some complications associated with pile driving and high damping, however, these materials can be a great option in aggressive corrosive environments. Concretefilled FRP tubes were used as a substitute to precast piles for Route 40 highway bridge over Nottaway River in Virginia (Fam et al., 2003). FRP pipe piling has also been used to replace the timber piers of Statue of Liberty Dock in San Francisco; another example is substitution of reinforced concrete piles in Long Beach pedestrian bridge and Legacy Dock piles. Most past studies on FRP piles were focused on the load transfer and flexural response of FRP piles under static loading. However, behaviour of FRP piles under seismic loading has not been well studied.

The behaviour of pile foundations under earthquake loading is an important factor affecting the performance of many essential structures. Analysis and design procedures have been developed 
for evaluating pile behaviour under earthquake loading. The application of these procedures to cases involving soft or liquefied ground is uncertain, however, due to both a lack of physical data against which they can be evaluated, and the continued lack of understanding of the mechanisms involved in soil-pile-structure interaction. Therefore, it is a critical measure to determine these uncertainties in order to avoid catastrophe in future earthquakes. This chapter reviews several past earthquakes, performance of different pile foundations systems under seismic loading, and eventually, a general overview on the building codes to consider the effect of the soil-foundationstructure interaction. Additionally, a summary of past research activities and experimental studies on load transfer, and seismic response of traditional piles and FRP piles is provided. Moreover, previously conducted experimental works in this area are explained aiming to be employed as a platform for the experimental shaking table investigations in this study.

\subsection{Pile Performance in Past Earthquakes}

There are number of past earthquakes that revealed performance of pile foundations in soft clay and liquefiable soils. Past studies indicated that there are weaknesses in pile performance during strong shakes with awareness modes of pile behaviour and failure. The following summary of case histories is presented as a representative survey of observed pile damage and failure during major earthquakes.

In the 1906 San Francisco Earthquake, the most intense damage was concentrated at the margins of the historic shoreline, which had been reclaimed with loosely dumped fill consisting of dune sand and silty sand in 1850. Beneath this fill is soft Bay Mud, which in turn is underlain by much stiffer cohesive deposits. Due to the strong shaking, an extensive development of liquefaction in sandy fill occurred, which caused foundational and subsequently structural failures (Seed et. al., 1990). Some of the modern buildings had damages throughout their concrete pile 
casings, and a number of damages were also occurred due to lateral spreading that caused the failure of timber piles that supporting bridges and railroad bridges (Figures 2.1 and 2.2). The deformation of the timber piles varied across the bridges due to distribution of loose and firm soil around the shore. The piles in firm shore were inclined proportionally whereas in muddy regions, the timber piles were inclined randomly (Wood, 1908).

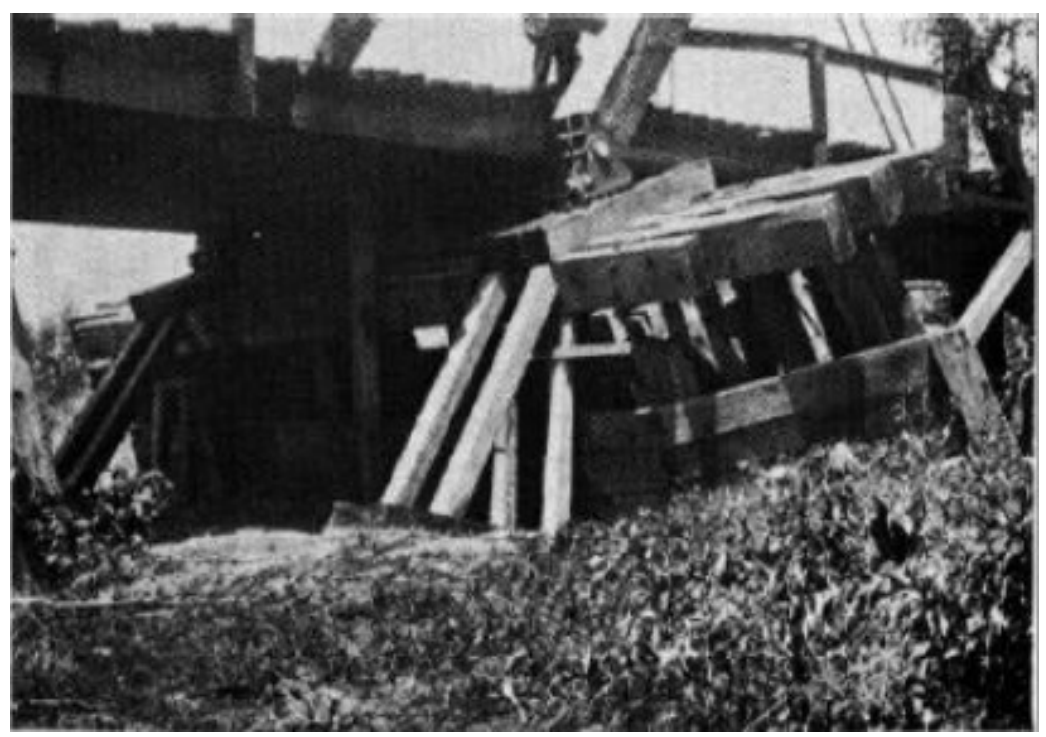

Figure 2.1: Failure of pile supported pier of the Salinas (after Wood, 1908)

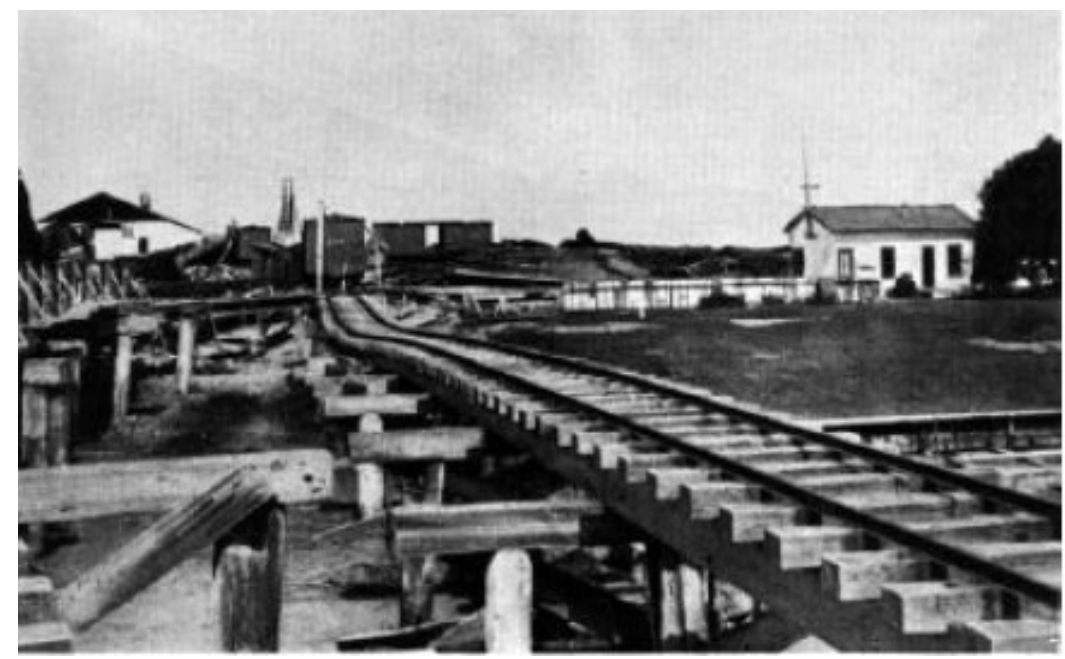

Figure 2.2: Timber Pile failure at Moss Landing (after Wood, 1908)

The Alaska Earthquake in 1964 is another example with wide-ranging damage to the foundation of the highway bridges which mostly occurred due to liquefaction of the foundation 
soils (Ross et al., 1973). Based on the observations, some of the timber piles that were driven to bedrock, showed minor damage, though there were significant settlements of the bridge abutments (Figure 2.3). Also, an under-construction bridge experienced liquefaction-induced failure of concrete-filled steel pipe piles, and caused the bridge pier to be shifted and/or failed.

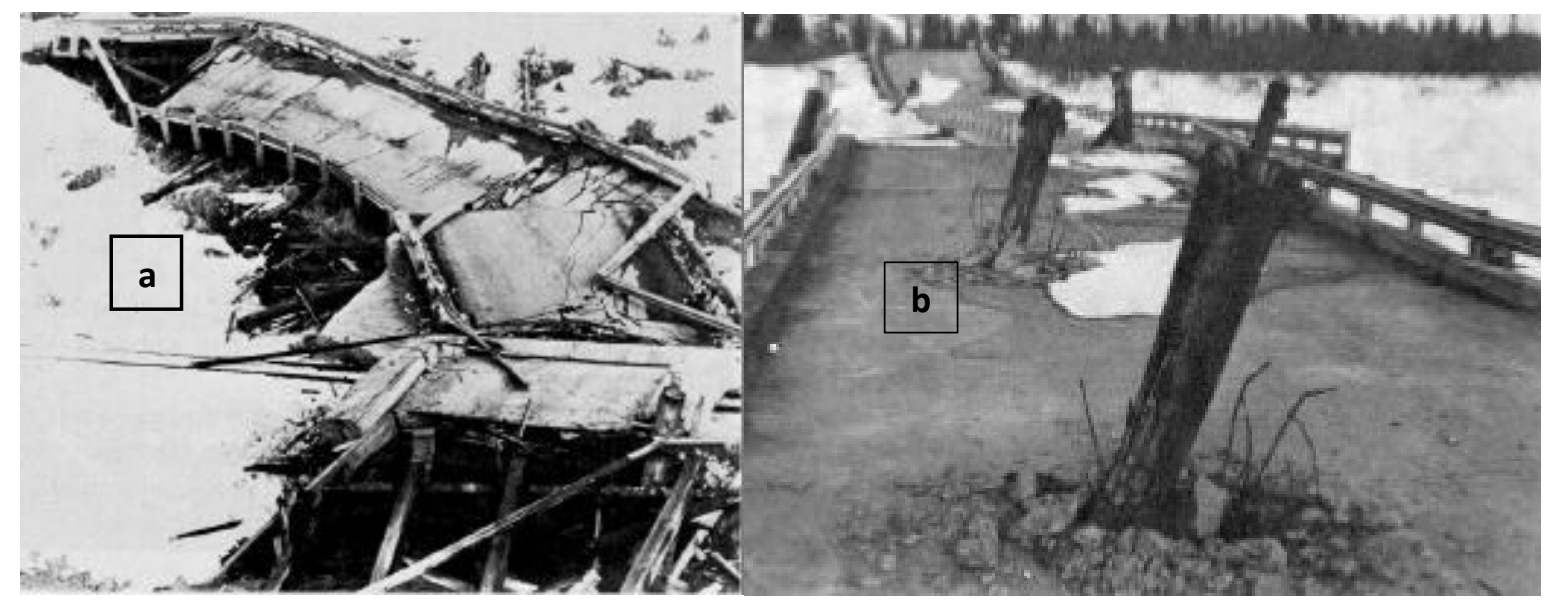

Figure 2.3: (a) Bridge 605 failure due to liquefaction in the Alaskan Earthquake, 1964 (Ross et al., 1973); (b) Timber piles penetrated through the concrete deck of Bridge 629 in the Alaskan Earthquake,1964 (Ross, et al., 1973)

The magnitude 7.8 Off-Tokachi Earthquake in 1968 and magnitude 7.4 aftershock caused substantial damage to a region of northern Japan. From the failures, the earthquake-induced vibration of the superstructure was the major reason to cause failure at the pile head though none of the failures was as a result of the liquefaction since the soil profile varied in ranges from sand, silt, clay, and even peat (Meymand, 1998). Mizuno (1987) stated that these failures forced Japanese engineers to re-consider and study the pile failures under dynamic loading in different case studies in order to understand the failure parameters for every case including, lateral deflection of cohesive soil in a uniform deposit, and lateral spreading of embankment, induction of liquefaction in uniform sandy deposit, shaking outcome of the soil, and inertial forces of superstructure that may cause pile damages and/or failures. Furthermore, the failure patterns were defined to recognise the weaknesses including shear and compressive failure of pile head, cracks 
due to bending moment, disengagement of pile cap and the pile, and connection failure of the welded joints.

The 1985 Mexico City Earthquake with a magnitude of 8.1 occurred approximately 400 km away from Mexico City, but a convergence of site response factors resulted in massive damage on the Lake Zone of Mexico City. The seismic waves effectively filtered to long period motion in travelling from the epicentre to the Mexico City region. In the Lake Zone, due to the existing deep soft clay deposit, the intensity of the shaking greatly amplified with a resulting period of approximately two seconds. This long period motion induced resonance for many structures of intermediate height, resulting in a damage pattern closely focused on these long period structures. Consequently, buildings supported by frictional pile foundations experienced the most damages (Meymand, 1998; Nghiem, 2009). Girault (1986) reported 25 buildings on mat foundations supported by frictional piles that experienced large settlement and tilting. These settlements occurred mainly because of reduction in the negative skin friction of the piles due to a partial loss of the shear strength of the soil during cyclic loading. Mendoza and Romo (1989) explained that low factor of safety against bearing capacity and a soil-pile stress state close to yielding with respect to static loading induced foundation failure under seismic loads. Figure 2.4 illustrates an overturned ten storey building, where one corner sank 6 meters into the soil and the opposite corner raised approximately 3 meters out of the soil, which pulled piles out of the ground.

During the 1985 Mexico City Earthquake, structures supported by the end-bearing pile foundations had a better performance during seismic excitation in comparison to the structures resting on frictional pile foundations. Meymand (1998) mentioned that the seismic overturning moments were the main reasons of failure of the pile foundations, however, some cyclic strength degradation can be also contributed to the partial loss of the soil-pile adhesion. The observations 
from 1985 Mexico City Earthquake are extremely important in terms of the seismic soil-pilestructure interaction, since the existing deep soft clay extended the period of the ground motion due to the site response.

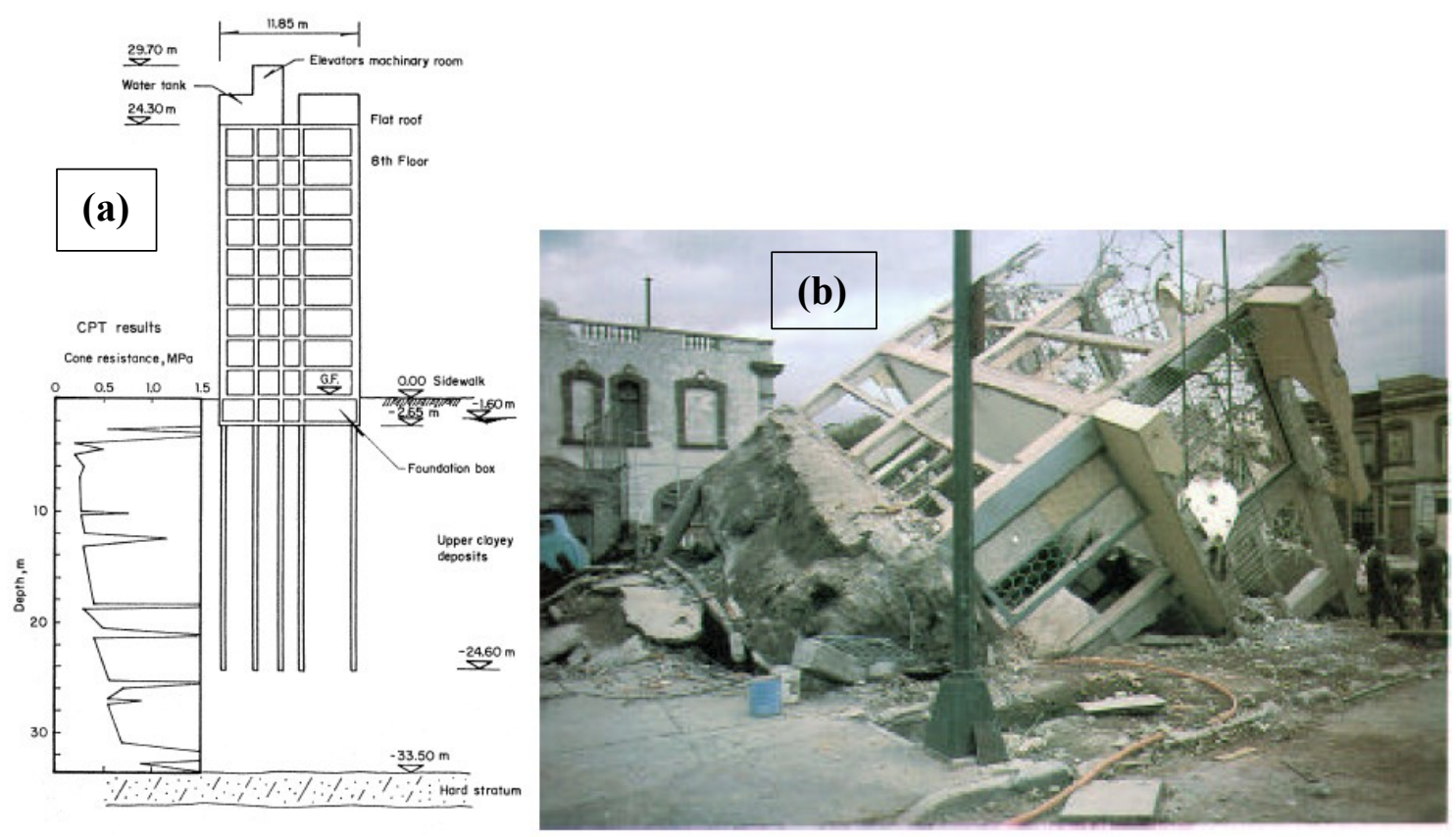

Figure 2.4: Ten story building supported by pile foundations in soft soils deposits during the 1985 Mexico City Earthquake: a) Site conditions; b) Overturned structure (after Mendoza and Auvinet, 1988)

The 1989 Loma Prieta Earthquake with magnitude 6.9 occurred in the San Francisco and Monterey Bay regions and caused extensive damage to many structures. The earthquake was responsible for 67 deaths and about 7 billion dollars in damage. Strong shaking from the earthquake occurred for 10-15 seconds over a wide area, resulting in high levels of damage near the epicentre (U.S. Geological Survey, 1990). As a result, ground motion amplification due to local site effect and insufficient lateral support of piles caused the collapse of highway bridges, port facilities and marine structures supported by the pile foundations driven into the soft soil specifically San Francisco Bay mud. The failure outcome was 30-45 cm gap between soil and pile due to the cyclic degradation of the pile's surrounding soil, contributing to the total collapse of the 
highway bridge as shown in in Figure 2.5. The insufficient support led to lateral displacement of the piles followed by the flexural and shear failures at the pile connections.
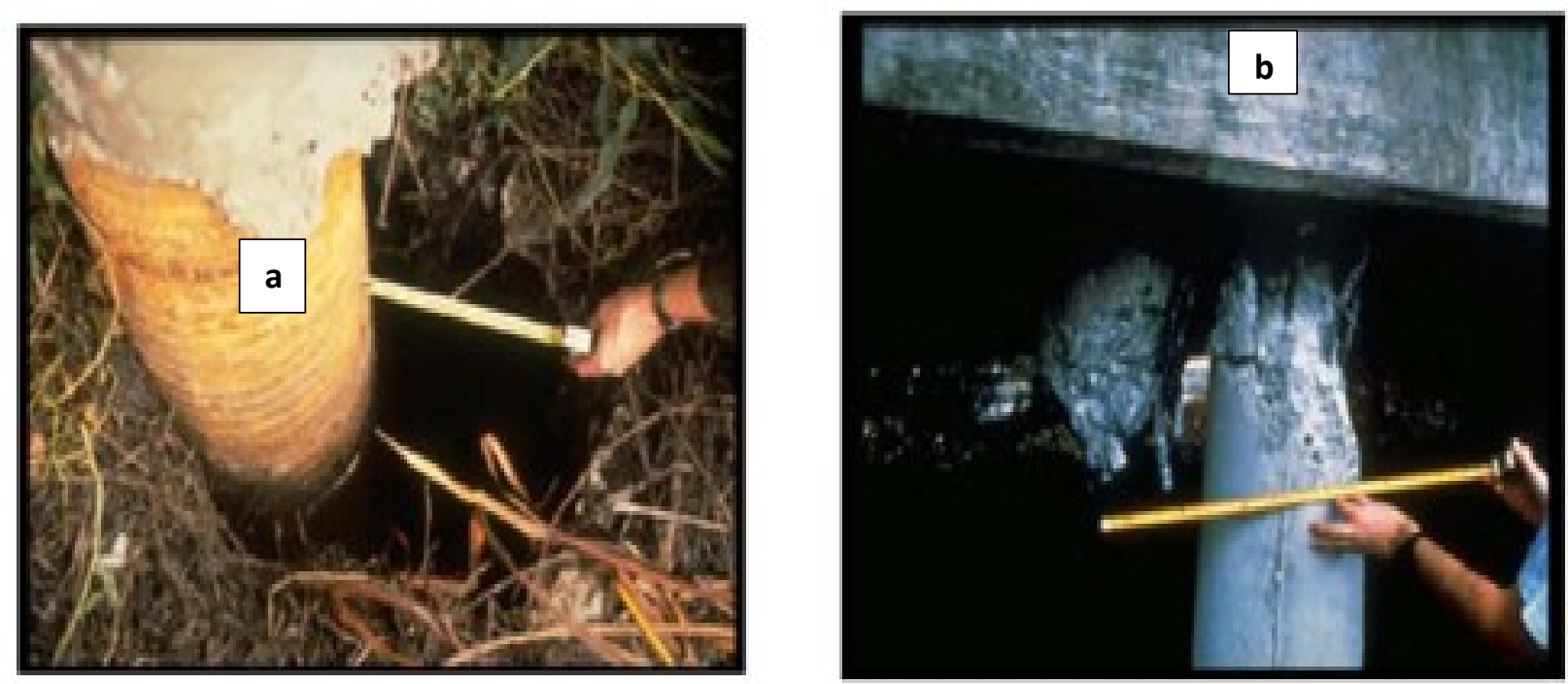

Figure 2.5: (a) Development of gap around the pile; (b) Flexural failure of pile supporting cross bridge during Loma Prieta earthquake (after Seed et al., 1990)

The 1964 Niigata Earthquake is an important example of earthquakes that raised the alertness between geotechnical engineers of earthquake-induced liquefactions and its disastrous significance. The epicentre was about 55 kilometres north of the city (offshore) and the recorded magnitude was 7.3 on the Richter scale. The city is underlain by about $30 \mathrm{~m}$ deposits of alluvial sand. Major damages appeared in areas where failure was due to the liquefaction of loose sand. Figure 2.6 shows a well-known failure of an apartment building on the sands sloped on side due to bearing capacity failure in the liquified ground. 


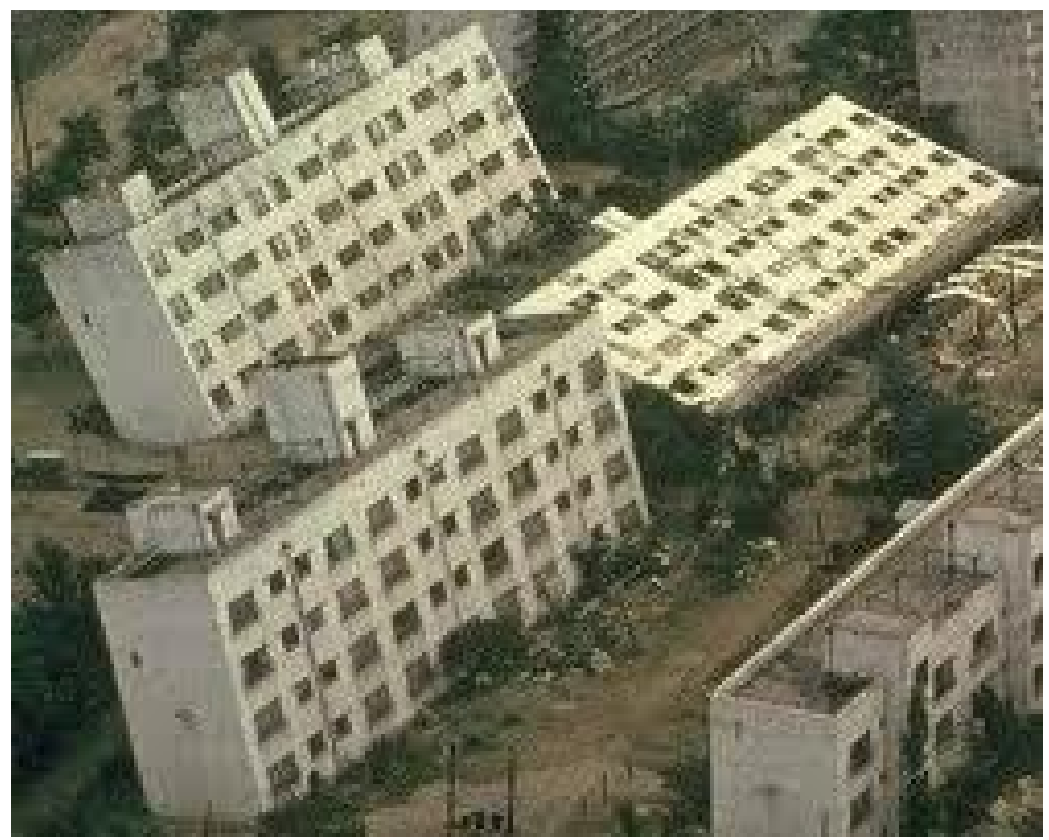

Figure 2.6: Bearing failure of foundation in Niigata Earthquake, 1964 (United States Geological Survey)

The Hyogoken-Nambu (known as Kobe Earthquake) Earthquake in 1995, with the magnitude of 7.2 and depth of $20 \mathrm{~km}$ was one of the most destructive earthquakes in Japan which most of destructions were due to foundation failures including pile foundations for highway bridges, buildings, tanks, etc. One of these major destructions was Hanshin Expressway in Higashi-nada which was collapsed and overturned. The bridge was part of an elevated expressway that runs parallel to the shoreline. The structure was founded on a group of 17 piles with a length of about $15 \mathrm{~m}$ and a $1 \mathrm{~m}$ diameter and rigidly connected to a $11 \mathrm{~m}$ x $11 \mathrm{~m}$ pile cap. The bridge contained of 18 spans which all failed during the earthquake. The soil in the area around the expressway is primarily made up of various layers of sand and gravel underlain by soft rock. The soil layers amplified the incoming seismic waves which in turn magnified the forces acting on the bridge (Gazetas and Mylonakis, 1998). 


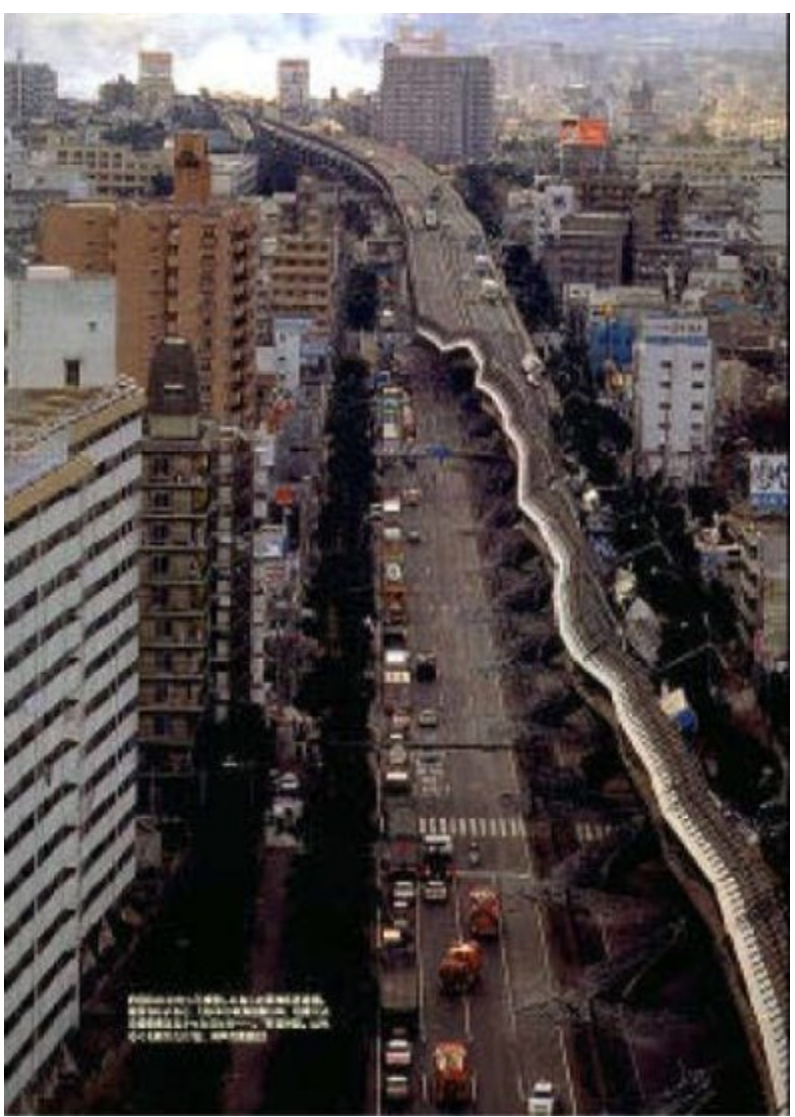

Figure 2.7: Hanshin Highway Bridge failure during the 1995 Kobe Earthquake (after Gazetas \& Mylonakis, 1998)

Several researchers investigated the damage on pile foundations through comprehensive soil investigations, in-situ measures on damaged piles, and several field and laboratory experiments to understand the failures. Gazetas and Mylonakis (1998) provided an analysis suggesting that period lengthening due to foundation flexibility can lead to an increase in structural forces during the earthquake. The fundamental period of the system assuming fixed-base pier and accounting for the rotational inertia of the deck was obtained to be about 0.65 second (Veletsos and Meek, 1974). Gerolymos (1997) determined the period accounting for soil-structure interaction (SSI) and found it to be about 0.93 seconds. The acceleration histories anticipated for the deck with and without the effects of SSI discovered different peaks and frequency behaviours. 
The system response considering SSI was $25 \%$ higher than the response for a fixed-base, confirming that the SSI may have been a contributing factor to the failure of the bridge.

The Chile Earthquake in 2010 with the magnitude of 8.8 was the sixth largest earthquake ever to be recorded by seismographs. Gibson (2010) detected that most buildings performed well under the strong shake with minor interior damages. However, some of high-rise buildings were damaged more than houses due to strong amplification of soft soil. This encouraged some of the researchers such as Bonelli et al. (2012) to offer design code evaluation for considering the effect of soil-structure interaction mechanism.

As stated by Meymand (1998), the following outline can be summarized based on the observed seismic performance of piles during earthquakes:

- From the instrumented cases, it is observed that seismic soil-pile-structure interaction often results in spectral de-amplification of pile cap motions relative to the free-field motion.

- Soil modulus degradation and soil-pile gapping may prevent radiation damping, particularly at the high level of shaking, which in turn dominates the structure inertial forces reducing the overall effects of the spectral de-amplification.

- Strain softening of cohesive soils or liquefaction of cohesionless soils near the pile head results in the loss of bearing capacity. The combination of this effect with the induced rocking mode in the superstructure results in the pile group settlement, punching failure, and/or tensile pull-out failure.

\subsection{Seismic Soil-Structure Interaction (SSI)}

The performance of pile foundations under seismic loading has been researched since the early 1970s, and the results of these studies and research have formed a considerable level of understanding. Under seismic excitation, a pile undergoes stresses both as a result of the vibration 
of the surrounding soil (kinematic interaction) and that of the superstructure (inertial interaction). Evaluating these interactions is a crucial issue in the seismic design of piles and pile-supported structures, several of which experienced considerable damage during previous devastating earthquakes.

The interaction of soil-structure has a major influence on structures response under dynamic loads. In other words, the response of the soil affects the motion of the structure and similarly, the response of the structure can affect the motion of the soil. It is essential to consider the consequence of SSI on structures specifically in soft soils and additionally, the failure of the foundation system to resist soil deformations and avoid disastrous damage. Several observations and studies have shown that the SSI effect often was the major reason for foundation and followed by structural failures. Some of these case studies showed failure due to liquefaction of cohesionless soils, however, in some cases the failures were reported for structures founded on soft and/or sensitive clay. Past studies have provided valuable information to understand the effects of SSI, although the case studies have not reached a point to finalize this phenomenon. It is difficult to recognise the soil flexibility by observation of seismic response of a structure; therefore, it needs much complicated identification devices and monitoring systems to identify the motions, both translational and rotational components, at the base and throughout the foundation. There are two discrete mechanisms that can affect the base shear demand: inertial interaction and kinematic interaction. Kinematic Interaction occurs due to the presence of the stiff shallow foundation or pile foundation elements in the soil resulting in the deviation of the foundation motion from the free field ground motion. Kinematic interaction could be due to the ground motion incoherence, foundation embedment effects, and wave scattering or inclination (Stewart et al., 1999a). This effect can be theoretically modelled by frequency-dependent ratios of the Fourier amplitudes of 
the foundation input motion to the free field ground motion refer to as transfer functions. The foundation input motion is the theoretical motion of the base slab if the base and the structure were massless. This foundation input motion presents more appropriate motion for the structural response analysis than the free field ground motion (FEMA, 2005). Inertial Interaction results from the inertia developed in the structure due to its own vibration producing base shear, moment and torsional excitation. These loads in turn cause displacements and rotations of the foundation relative to the free field condition (Kramer and Stewart, 2004). The displacements and rotations of the foundation can be described by Impedance functions representing frequency-dependent relationships between forces and moments applied to the foundation and the corresponding displacements and rotations of the foundation relative to the free-field motion.

The kinematic interaction can occur due to the presence of rigid foundation elements within the soil that causes to induce foundation motions to deviate from free field motions. Three mechanisms can potentially contribute to such deviations: (a) embedment effect is defined as frequency dependent transfer function involving the free field motion that can appear on the base slab while the base and structure are massless. In general, the seismic ground motion decreases with depth for embedded foundations.

Another potential deviation identified as base-slab averaging (b) which is defined as freefield motions associated with inclined and/or incoherent wave fields that are "averaged" within the footprint area of the base-slab due to the kinematic constraint of essentially rigid-body motion of the slab. (c) Wave scattering describes scattering of seismic waves of corners and severities of the foundation. The outcomes of these phenomena are often described by a complex-valued transfer function correlating free-field and foundation motions, and a complex-valued impedance function which quantifies the stiffness and damping characteristics of foundation-soil interaction. The 
damping represented by the imaginary part of the impedance function is a consequence of hysteretic damping in the soil and foundation, and radiation of seismic energy away from the foundation through the soil. Both the transfer and impedance functions are dependent on the finite stiffness and damping characteristics of the soil medium. For the fictional condition of an infinitely stiff soil, the amplitude of the transfer function for translational motion is unity and the phase is zero (i.e., the foundation and free-field motions are identical), and the impedance function has infinite real parts and zero imaginary parts. It is of some practical significance that this unrealistic assumption of rigid soil is made when SSI effects are ignored (which is common practice in structural design).

On the other hand, the inertial interaction occurs due to generation of inertia from the structure, also known as vibration. This results in excitation of the base shear, moment and torsion, that can appear as displacement and rotation of the foundation relative to the free field. Accordingly, these types of response are related to the soil-structure system compliance that can be essentially associated to the structural flexibility. Additionally, the relative foundation-free field motions increase as energy dissipation through radiation that can essentially affect the damping of the system (Kramer and Stewart, 2004). Kinematic and inertial responses occur instantaneously with only a minor time delay, but the effects of inertial interaction are generally more pronounced compared to the effect of kinematic interaction. However, the total response of the system is completed by superimposing the two responses of inertia and kinemetric interactions (Figure 2.8). 


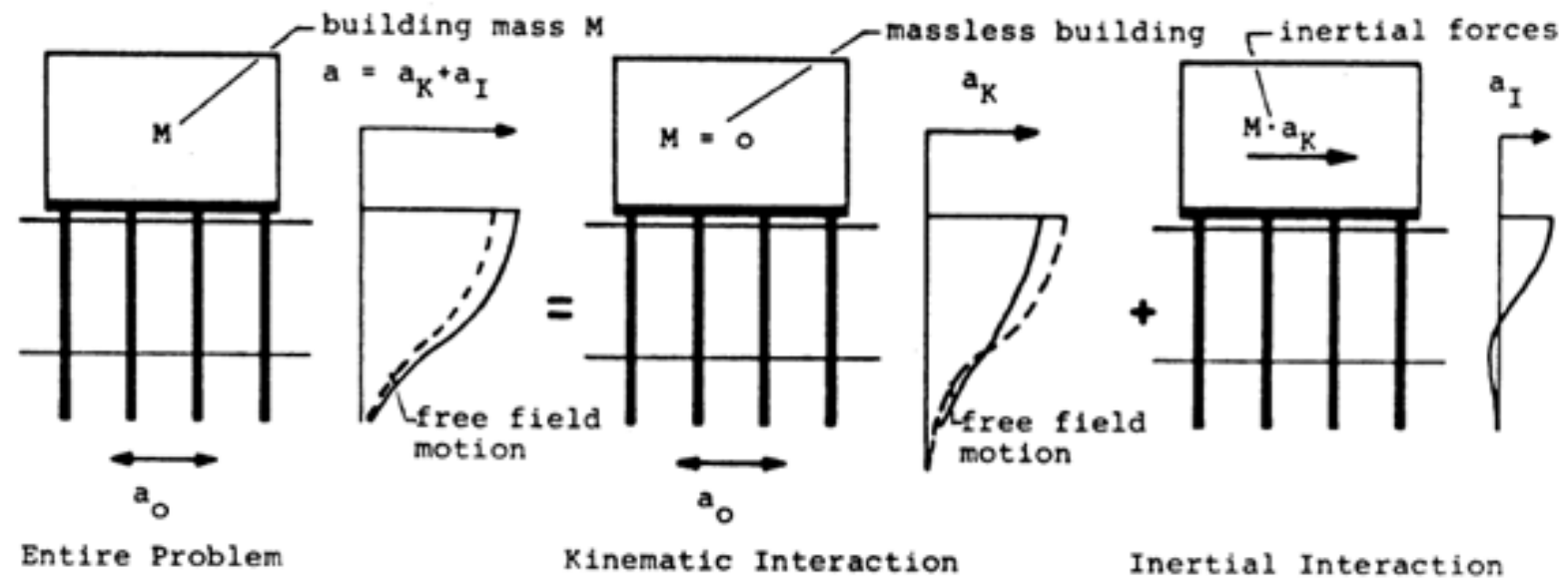

Figure 2.8: Separation of SSPSI Analysis into Kinematic and Inertial Interaction Components (after Waas and Hartmann, 1981)

\subsection{Effect of SSI on Foundation and Structural Response}

The response acceleration generated by structures is affected by the foundation input motion and the flexibility of foundations. SSI affects the dynamic behaviour of the structure through changing the natural frequency and shaking level of the soil-structure system to a value different than that of the structure under fixed-base conditions (i.e., when structure rests on rock). The response can eventually cause an increase in structural drift, thus the SSI can change the effective damping ratio to larger values compared to structure's damping ratio. This may decrease the maximum structural distortion and reach higher displacement which is inversely proportional to soil stiffness. Therefore, the structural demand can decrease due to higher flexibility of the foundation system, though the result would be higher total displacement. This type of performance is important for tall and slender structures or buildings that are spaced close to each other since large relative displacement can be generated due to pounding.

The effect of SSI can be significant enough to consider for intermediate to lengthy period structures. The effect of SSI needs to be considered at any maximum acceleration with a period 
larger than 1.0 second. A number of researchers stated that SSI mechanism can be also essential for short period structures (Veletsos, 1989; Balendra and Heidebrecht, 1986). Two past earthquake examples, the 1985 Mexico City and the 1995 Kobe revealed peak accelerations at a period greater than 1.0 second, and a portion of the damage originated at these locations were due to the SSI effects. Therefore, any increase of fundamental period due to SSI can cause an increase in response to cross over the boundary of standard design spectra for a region. Accordingly, soil-structure interaction can generate amplification or even reduction of the base shear, depending on the spectral period at which the response spectra with and without interaction cross each other. Therefore, structures with a fundamental period larger than the prevailing site period may experience reduction in spectral acceleration (Rayhani, 2007).

Kim and Roesset (2004) found out that as the soil softens and develops nonlinear behaviour, the natural period elongates and affects the peak acceleration due to amplification which this represents the inelastic seismic response of structures. These results illustrated the necessity of accounting for nonlinear soil behaviour on the response of structures. However, the effect of SSI can be insignificant for flexible structures on stiff soils, though it is essential for stiff structures on soft soils.

\subsection{Pile Foundation Design under Seismic Loads}

Seismic design of pile foundations is often conducted based on the single soil-pile interaction analyses and then it is extended to reveal the group configurations effects to achieve full SPSI analyses. An essential factor in group interaction can be attributed to group arrangement and pileto-pile spacings which the latter may be ignored for large spacings (i.e., 6-8 time of pile diameter) (Bogard and Matlock, 1983; Hokmabadi, 2014). However, this cannot be a suitable method when piles experience seismic loadings where pile behaviour can be impacted by the wave energy 
reflected between adjacent piles. Pile group effects were also shown to reduce with increasing soilpile nonlinearity, which this may consequently prevent wave transmission among piles (Meymand 1998). When exposed to cyclic loading, pile group effects were discovered to cause the degradation of soil resistance in stiff clay soils, while, there was no loss of capacity in sandy soils due to soil densification (Brown et al. 1987, 1989). Brown et al. (1988) also reported greater bending moment fore pile in a group and showed that pile group deflection surpassed single pile deformation (Fig. 2.9).
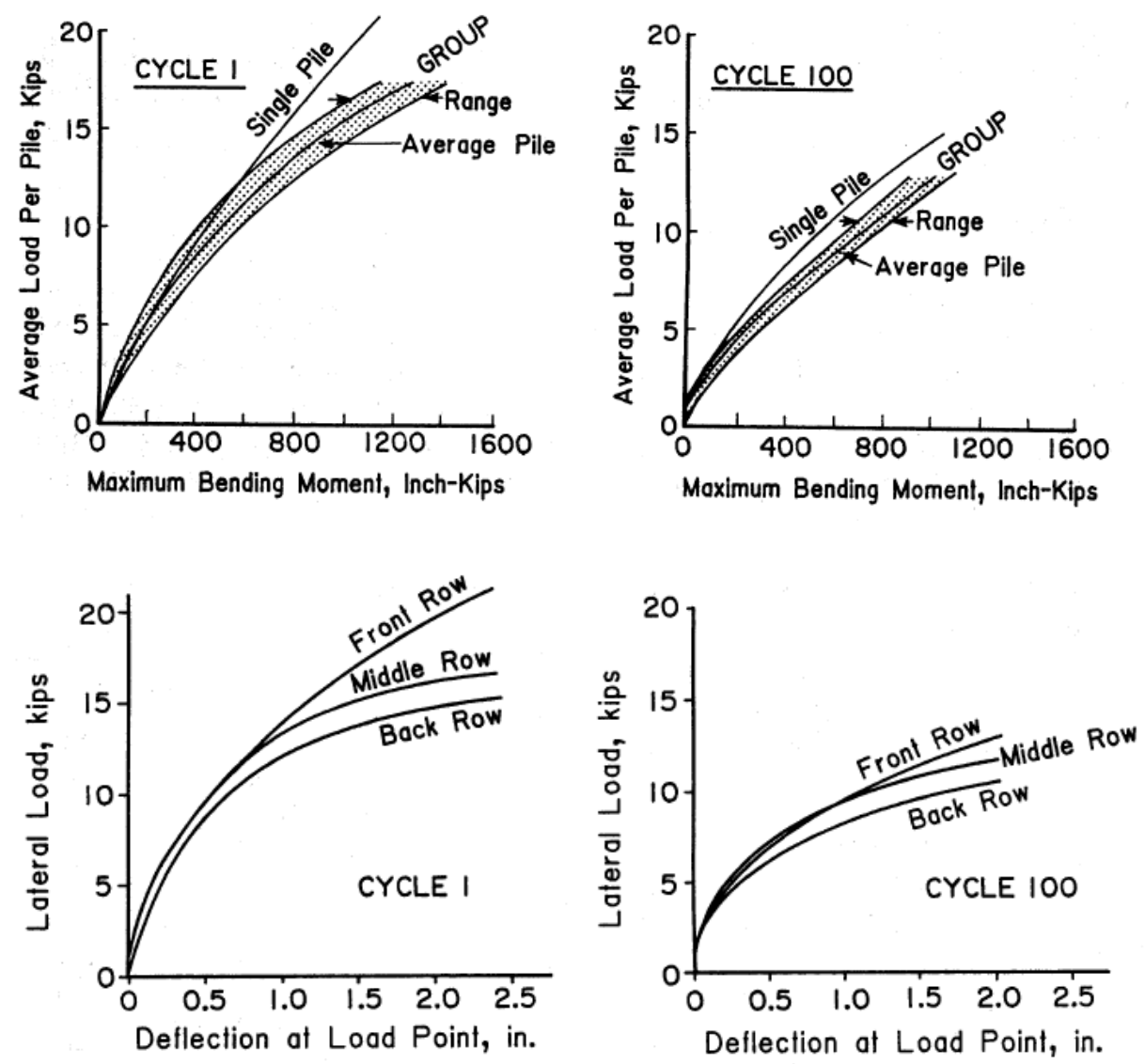

Figure 2.9: Results of lateral response of steel pile group: a) Cyclic degradation of resistance; b) Load distribution within group in each row (after Brown et al., 1987) 
Several researchers have analyzed the seismic soil-pile structure interaction (SPSI) phenomenon and its influence on the response of various structures by developing analytical methods, which can be divided into three main groups: (1) Beam on Elastic Foundation Methods (or Winkler methods); (2) Elastic Continuum Methods; and (3) Numerical Methods (i.e., FEM).

\subsubsection{Beam on Elastic Foundation Method}

The beam on elastic foundation method is known as the Winkler's theory which assumes that each layer of soil responds independently to the applied pile load through a series of springs and dashpots that are utilized to characterize the soil behaviour around the pile. Hetenyi (1946) developed the initial governing expression for beam-on-elastic-foundation solutions as follow:

$$
E I\left(\frac{d^{4} y}{d x^{4}}\right)+P_{x}\left(\frac{d^{2} y}{d x^{2}}\right)+E_{S}(y)=0
$$

where, $E I$ is the flexural stiffness of pile, $P_{x}$ is the axial load, $y$ is the lateral deflection of the pile at point $x$ along the length of the pile, and $E_{s}$ is the spring's stiffness (i.e., soil stiffness). Matlock and Reese (1960) suggested a comprehensive iterative solution method for rigid and flexible laterally loaded piles where linear or nonlinear soil-pile springs can be utilized to analyse SPSI. Since, soil material can exhibit nonlinear stress-strain behaviour from very narrow strain ranges, and modelling of soil-pile interaction involves nonlinear springs to reflect subgrade reaction and time domain formulation of equation of motion to capture time-varying interaction properties. During the last decades, load-deformation ( $\mathrm{p}-\mathrm{y}$ ) curves have also been developed to understand piles behaviour under lateral loading in in sand and clay mediums (e.g., Cox et al.,1974; Reese et al.,1975; Gazioglu and O’Neill, 1984). 


\subsubsection{Elastic Continuum Method}

The elastic continuum analytical methods are based on a closed form solution proposed by Mindlin (1936) for the application of point loads on a semi-infinite elastic medium. These solutions mostly rely on elastic parameters of the soil and may not be suitable to integrate the soil nonlinearity, however, they can be adopted for small strain and steady state problems. Poulos (1971) introduced the concept of pile group interaction factor using Mindlin's elasticity equations to solve for stresses and displacements between pairs of piles due to horizontal point loads applied in an elastic half space. The described interaction factors can be described as:

$$
\alpha=\frac{\text { additional displacement due to adjacent pile }}{\text { displacement of pile due to its own loading }}
$$

where, $\alpha$ is presented as a chart with factors for both fixed and free head piles subjected to lateral and moment loadings as functions of pile flexibility $K_{r}$, which is defined based on pile spacing, pile diameter, pile length, and departure angle (angle between piles and direction of loading). This analysis considers superposition principle by estimating individual interaction of piles compared to others in same group, however, this method may underestimate or overestimate pile group interaction depending on small and/or large spacing, respectively. Gazetas (1991) proposed detailed design charts and impedance functions for direct estimation of pile cap lateral and axial stiffness and damping coefficients representing the soil profile. Stewart (1997) stated sophisticated models can be only viable option to study the SPSI problem, however simplified models can still deliver some analytical understanding through the contribution to lateral and rocking stiffness of pile foundations. 


\subsubsection{Numerical Methods}

The extensive capability of computational methods has increased the opportunity to conduct time history analysis on pile foundations considering various effects such as nonlinear stress-strain behaviour of the soil and its superstructure, material stiffness and radiation damping, advanced boundary conditions and interface elements. This allows to perform and understand the SPSI analysis on pile groups in a fully-coupled manner without altering to independent calculations of site and superstructure response, or application of pile group interaction factors (Meymand, 1998). Numerical approaches such as the finite element method (FEM) and the finite difference method (FDM) use an extended type of matrix analysis that divides the entire system into a finite number of elements connected at different nodal points. While finite element programmes often combine element matrices into a large global stiffness matrix, this is not always the case with finite difference programmes rather more efficient approach is taken to regenerate the finite difference equations at each stage (Desai and Abel, 1972).

The modelling technique for SPSI analysis are typically categorized into following two principles of substructure and direct methods. (i) In the Substructure method, the SSI problem is divided into two cases of near-field which simulates the SSI interaction, and the far-field is referred to nature of the soil medium as a semi-infinite for the site response. In this method, assessment of foundation input motion (FIM), as a motion that occurs on the base-slab, is conducted with the assumption that the structure and foundation have no mass. The dynamic analysis of the structure rested on base is carried out using the impedance functions subjected to dynamic excitation of the input motion. In the direct method, the entire soil-pile-structure system is modelled simultaneously, and the input motions are defined along the model's base. 
Torabi (2016) developed numerical and analytical frameworks to investigate seismic response of a representative bridge superstructure supported by a large-diameter pile shaft under fully coupled inelastic soil-pile-structure interaction using a 3-D finite element model of the soilpile system. The result from parametric study showed that the residual structural drift and the bending moment of the system would barely exceed the design limits. The key conclusion of this study showed that soil and interface inelasticity can significantly affect the impedance values compared to their fully elastic counterpart. Kwon and Yoo (2019) established a 3-D numerical model based on finite-difference to estimate the dynamic soil-pile-structure interaction (SPSI) in dry sand. Comparing the results, the inertial force induced by superstructure is dominant for dynamic SPSI in dry sand while the kinematic force generated by soil deformation was fairly insignificant. Additionally, the peak bending moment was found to occur with $30 \%$ of the pile length (pile models with length of less than $10 \mathrm{~m}$ ). The pile head fixity also affected the peak moment profile of pile and overall dynamic responses of the SPSI system.

Accordingly, numerical methods can be suitable alternative to validate the impacts of material nonlinearity (i.e., nonlinear stress-strain behaviour), stress anisotropy, radiation damping, and variations in soil medium in the dynamic response of SSI analysis. Hence, it is essential to consider appropriate mechanism in numerical analysis to evaluate dynamic response to achieve rigorous and realistic results (Bowles, 2001).

\subsection{Building Code Provisions for SSI}

This section reviews building code recommendations pertaining design of pile foundations and soil-pile interaction impact on piles' behaviour. Accordingly, most of these code recommendations are based on simplified soil-structure interaction analysis approaches, and hence, there is necessity for site specific studies for piles especially in soft soils exposed to strong levels of shaking. As per 
the design codes, seismic analysis of any structure supported on soft soil needs to consider the site effect and soil-structure interaction. The site effect specifies the scattering and diffraction of incident waves by the soil layer overlying bedrock. The soil-structure interaction is defined as the affiliation between characteristics of both the foundation and the soil layer, which this is signified by adjusting the dynamic properties of the structure. Generally, it is adequate to assume the site effects due to surface layering for code-designed buildings, and the effect of soil-structure interaction on the fundamental mode of vibration which is stated by an increment in the fundamental period of the structure and a variation in the correlated damping.

\subsubsection{National Earthquake Hazards Reduction Program}

The 2001 NEHRP Recommended Provisions for Seismic Regulations (BSSC, 2001) includes comprehensive measures for integrating the effects of soil-structure interaction in the specification of seismic design forces throughout structure. Result of these effects may reduce the base shear applied to the structure, and thus the lateral forces and overturning moments. It can also increase the lateral displacement due to rocking. The guideline is specifically applicable for new buildings which apply a force-based condition to its structural capacity and seismic demand that can be signified by a base shear force which is proportional to the product of structural mass and first mode of spectral acceleration. As well, inertial interaction is also considered through examination of a period lengthening ratio and damping factor of foundation that adjusts the base shear. Nevertheless, the guideline can be applied for a flat spectral shape at small periods, and it is restricted for flexible base damping to standards larger than the fixed base damping. The restriction may confirm that soil-structure interaction may only decrease the base shear demand. On the other hand, kinematic interaction is completely ignored thus it can reduce the seismic demand. 


\subsubsection{American Society of Civil Engineers (ASCE 7-10)}

The ASCE code designated "Minimum Design Loads for Buildings and Other Structures" (ASCE7-10, 2010) states that if the alternative to integrate the effects of the soil-structure interaction is exercised, the following method is acceptable to be used in the evaluation of the design earthquake forces and the corresponding displacements of the structure in the case that the model used for structural response analysis does not directly incorporate the effects of the foundation flexibility. Hence, this procedure cannot be used for flexible-base foundations. However, this method can only account for decreasing the base shear due to the soil-structure interaction corresponding to the fundamental (initial) mode of vibration by using the following equations:

$$
\begin{aligned}
& \tilde{V}=V-\Delta V \\
& \Delta V=\left[C_{s}-\tilde{C_{s}} \widetilde{\left(\frac{0.05}{\widetilde{\beta}}\right)^{0.4}}\right] \bar{W} \leq 0.3 V
\end{aligned}
$$

where, $\tilde{V}$ is the modified base shear, $V$ is the base shear of the fixed-base structure, $\Delta V$ is the reduction in the base shear due to the soil-structure interaction, $\mathrm{C}_{\mathrm{s}}$ is the seismic design coefficient estimated from the fundamental natural period of the fixed-base structure $(T), \tilde{C}_{S}$ is the seismic design coefficient computed using the fundamental natural period of the flexible-based structure $(\widetilde{T}), \tilde{\beta}$ is defined as the fraction of critical damping for the structure-foundation system, and $\bar{W}$ is defined as the effective seismic weight of the structure. It is also essential to estimate the effective period and stiffness of the structure due to the soil-structure interaction. ASCE code (2010) provides a spring-dashpot model with single degree of freedom of structure comparable as shown in Figure 2.10. The modified period of the structure is evaluated from the following Equation (2.5) 
which evidently highlights on the increase in the natural period of the system due to soil-structure interaction.

$$
\begin{aligned}
& \tilde{T}=T \sqrt{1+\frac{\bar{k}}{K_{y}}\left(1+\frac{K_{y} \bar{h}^{2}}{K_{\theta}}\right)} \\
& \bar{k}=4 \pi^{2}\left(\frac{\bar{W}}{g T^{2}}\right)
\end{aligned}
$$

where, $\bar{k}$ is the stiffness of the structure fixed at the base, $K_{y}$ is lateral stiffness of foundation which is defined as the horizontal force at level of foundation where it generates a unit deflection at that level, $K_{\theta}$ is rocking stiffness of the foundation which is defined as the essential moment to cause a unit average rotation of foundation, and ' $\mathrm{g}$ ' is the gravity acceleration.

There are no specific equations offered by the code to evaluate the lateral and rocking stiffness, however the ASCE code recommends estimating these parameters using the principles of foundation mechanics using soil properties. Additionally, several more parameters are needed including shear stiffness $(G)$, and shear wave velocity, $\left(V_{s}\right)$ which these variables are required to be compatible with the soil strain levels related with the design earthquake motion $\left(\mathrm{S}_{\mathrm{DS}}\right)$ as shown in Table 2.1. $V_{\text {so }}$ is defined as the average shear wave velocity for the soil deposit beneath the foundation (at strains $\leq 10^{-3}$ ); correspondingly, $G_{o}$ is the average shear modulus for the soil deposit beneath the foundation $\left(G_{o=} \gamma V_{2} S_{o} / g\right)$, and $\gamma$ is defined as the average unit weight of the soil deposit. The referred evaluation for seismic design and site classification only considers the upper $30 \mathrm{~m}$ of the site profile with regard to the soil properties such as the shear wave velocity. However, further research needs to be completed to justify the effect of kinematic interaction, the non-linear performance of the soil, and the effect of soil-structure interaction on higher modes specifically for high-rise structures. Therefore, the simplified linear spring models need to be 
developed in order to consider the impact of SSI under strong seismic motions in case superstructure rests on soft soils.

Table 2.1: Value of $\mathrm{G} / \mathrm{G}_{0}$ and $\mathrm{v} / \mathrm{v}_{\text {so }}(\mathrm{ASCE} 7-10,2010)$

\begin{tabular}{|c|c|c|c|c|c|c|}
\hline \multirow[b]{3}{*}{ Site Class } & \multicolumn{3}{|c|}{ Value of $v / \mathbf{v}_{\text {so }}$} & \multicolumn{3}{|c|}{ Value of $\mathbf{G} / \mathbf{G}_{0}$} \\
\hline & \multicolumn{3}{|c|}{$\mathrm{S}_{\mathrm{DS}} / 2.5$} & \multicolumn{3}{|c|}{$\mathrm{S}_{\mathrm{DS}} / 2.5$} \\
\hline & $\leq 0.1$ & 0.4 & $\geq 0.8$ & $\leq 0.1$ & 0.4 & $\geq 0.8$ \\
\hline $\mathbf{A}$ & 1.00 & 1.00 & 1.00 & 1.00 & 1.00 & 1.00 \\
\hline B & 1.00 & 0.97 & 0.95 & 1.00 & 0.95 & 0.90 \\
\hline C & 0.97 & 0.87 & 0.77 & 0.95 & 0.75 & 0.60 \\
\hline D & 0.95 & 0.71 & 0.32 & 0.90 & 0.50 & 0.10 \\
\hline $\mathbf{E}$ & 0.77 & 0.22 & $\mathrm{a}$ & 0.60 & 0.05 & $\mathrm{a}$ \\
\hline $\mathbf{F}$ & $\mathrm{a}$ & $\mathrm{a}$ & $\mathrm{a}$ & $\mathrm{a}$ & $\mathrm{a}$ & $\mathrm{a}$ \\
\hline
\end{tabular}

Note: Use straight-line interpolation for intermediate value of $S_{\mathrm{ds}} / 2.5$.

${ }^{a}$ should be evaluated from site specific analysis.
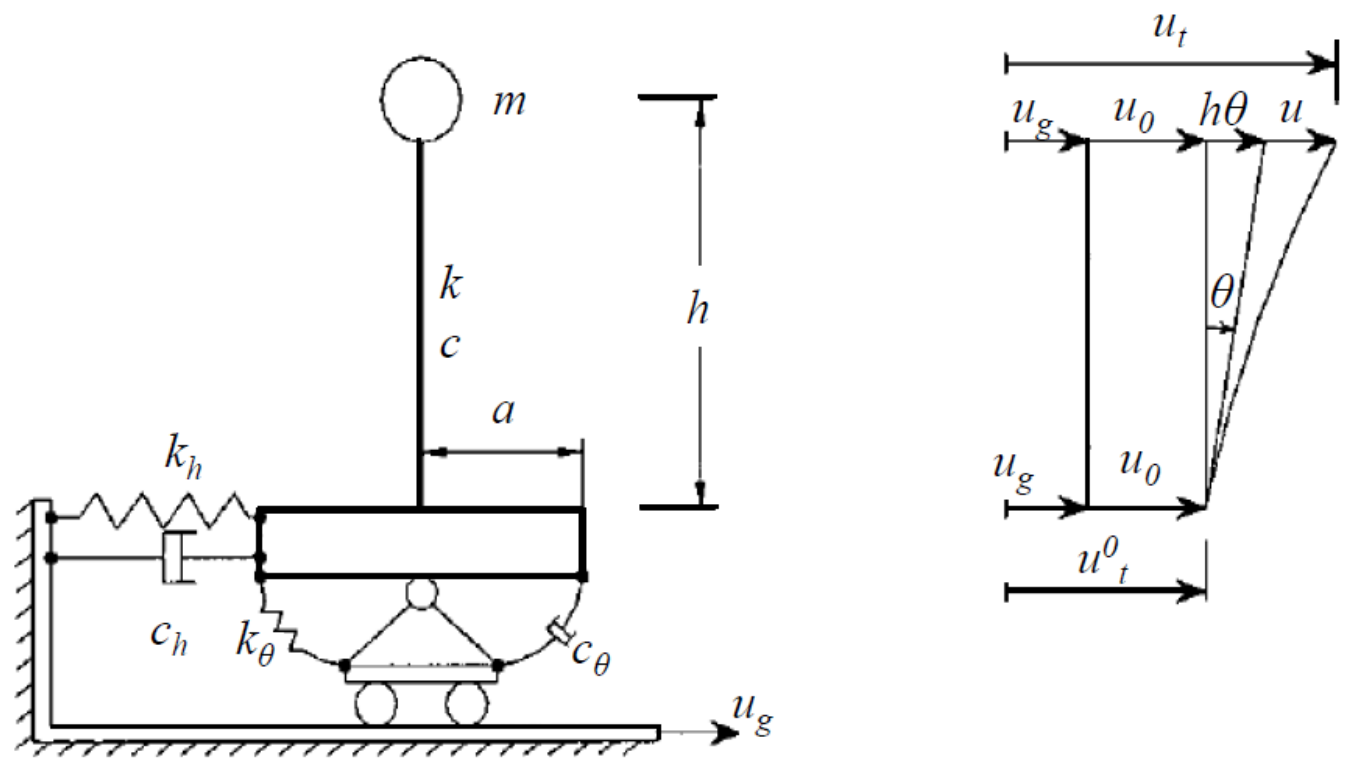

Figure 2.10: Soil-structure interaction model including a single degree of freedom (SDOF) structure and discrete system to signify the supporting soil (after Wolf, 1985) 


\subsubsection{National Building Code of Canada}

The 2015 National Building Code of Canada (NBCC) postulates the effect of soil-structure interaction on the seismic response of most buildings is favourable; hence it avoids the importance of this phenomena and recommends no guideline or approach to investigate the SSI. Accordingly, the seismic provisions of the proposed NBCC 2015 recommend applying soil-structure interaction analysis for critical or unconventional structures only. Soil-structure interaction effects are not recognized clearly in the provisions, which could be essential for several structures including high, slender structures or for closely spaced structures that may be subject to pounding at relatively large displacements occurrence.

\subsection{Mechanics of Composite Pile Materials}

Pile foundations are traditionally made of concrete, steel, timber, and composite materials. From 1987, several common types of composite piles have been developed including steel core piles, reinforced plastic piles, concrete filled Fibre-Reinforced Polymer (FRP) piles, fiberglass pultruded piles, fiberglass reinforced plastic piles, FRP hollow piles and FRP sheet piles (Figure 2.11). During the last two decades, FRP composite has become an alternative construction material without many of the performance drawbacks associated to traditional materials. Fibre reinforced polymers have been widely used for strengthening and repair of existing structures such as seismic retrofit and service load strengthening. The use of FRP as pile materials in practice has been limited to a few projects (e.g., Fam et al 1997). 


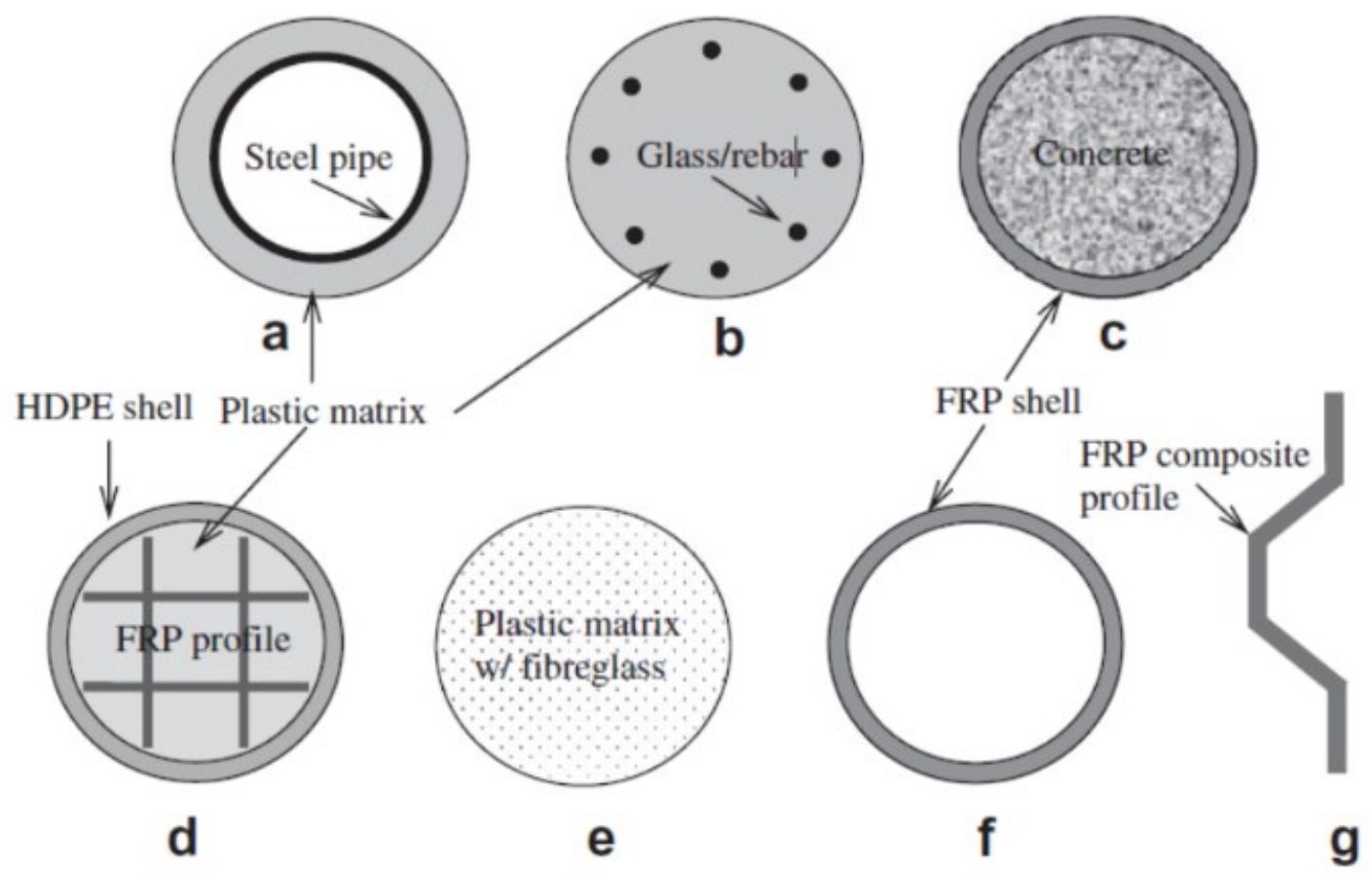

Figure 2.11: Geometric profile of acknowledged FRP piles in industry. (a) steel pipe core, (b) structurally reinforced plastic, (c) concrete-filled FRP, (d) reinforced plastic piles, (f) hollow FRP piles and (g) FRP sheet piles (Guades et al., 2012)

In most cases FRPs are used in the form of a structural component replacement such as FRP tendons or rebars for steel ones, or FRP I-beam for steel I-beam, which might not essentially be cost effective. The advantages of FRPs for civil engineering applications such as the lightweight and high strength can be utilized in various forms such as hollow FRP round tubes. The hollow FRP pile system utilizes the suitable characteristics of the individual materials. Also, the hollow FRP pile system simplifies construction procedures, and reduces manufacture time. This study mostly concentrates on hollow FRP piles in groups compared to traditional piles in groups in order to understand the dynamic behaviour of FRP piles and its interaction in marine environment and weak soils. The concept of hollow FRP pile was mainly developed to be used as non-corrosive piles for marine environment and waterfront applications. 


\subsection{Fibre-Reinforced Polymer (FRP)}

Fibre-reinforced polymers refer to composite materials consisting of two phases including a dispersed phase (i.e., reinforcement), and a continuous phase (i.e., matrix). The dispersed phase is typically continuous-fibre reinforcement, which is the load-carrying element of the composite material and controls the stiffness and strength. The matrix phase maintains protection and support for the sensitive fibres along with local stress transfer from one fibre to another. The continuous fibres can be formed in different orientation format including all parallel (i.e., unidirectional continuous fibre composites), oriented at right angle to each other (i.e., cross ply or woven fabric continuous fibre composites), and/or oriented along several directions (i.e., multi-directional continuous fibre composites). Polymer matrix composites include thennoset (combination of epoxy, polyimide, polyester) or thennoplastic (i.e., polyether ether ketone as colourless thermoplastic, polysulfone known as high performance thermoplastics), resins reinforced with glass (GFRP), carbon (CFRP), aramid (AFRP), or boron fibres (BFRP) (Daniel and Ishai 1994).

A lamina or a ply is an even or curved layer of unidirectional fibres or woven fabric in a matrix. The lamina is considered an orthotropic material with principal material axes $(1,2$, and 3$)$ as shown in Figure 2.12 (a). A laminate is manufactured in either two or more unidirectional laminae at various orientations. Meanwhile, the principal material axes differ from one ply to another, hence, it is more convenient to analyse laminates using a common fixed system of coordinates (x, y, and z) as shown in Figure 2.12 (b). The orientation of a given ply is given by the angle between $\mathrm{x}$-axis and principal axis-1 of the ply, measured positive in a counter-clockwise direction. The formation of the laminate indicating its ply composition and precise location of various layers is called stacking sequence (Daniel and Ishai 1994). 


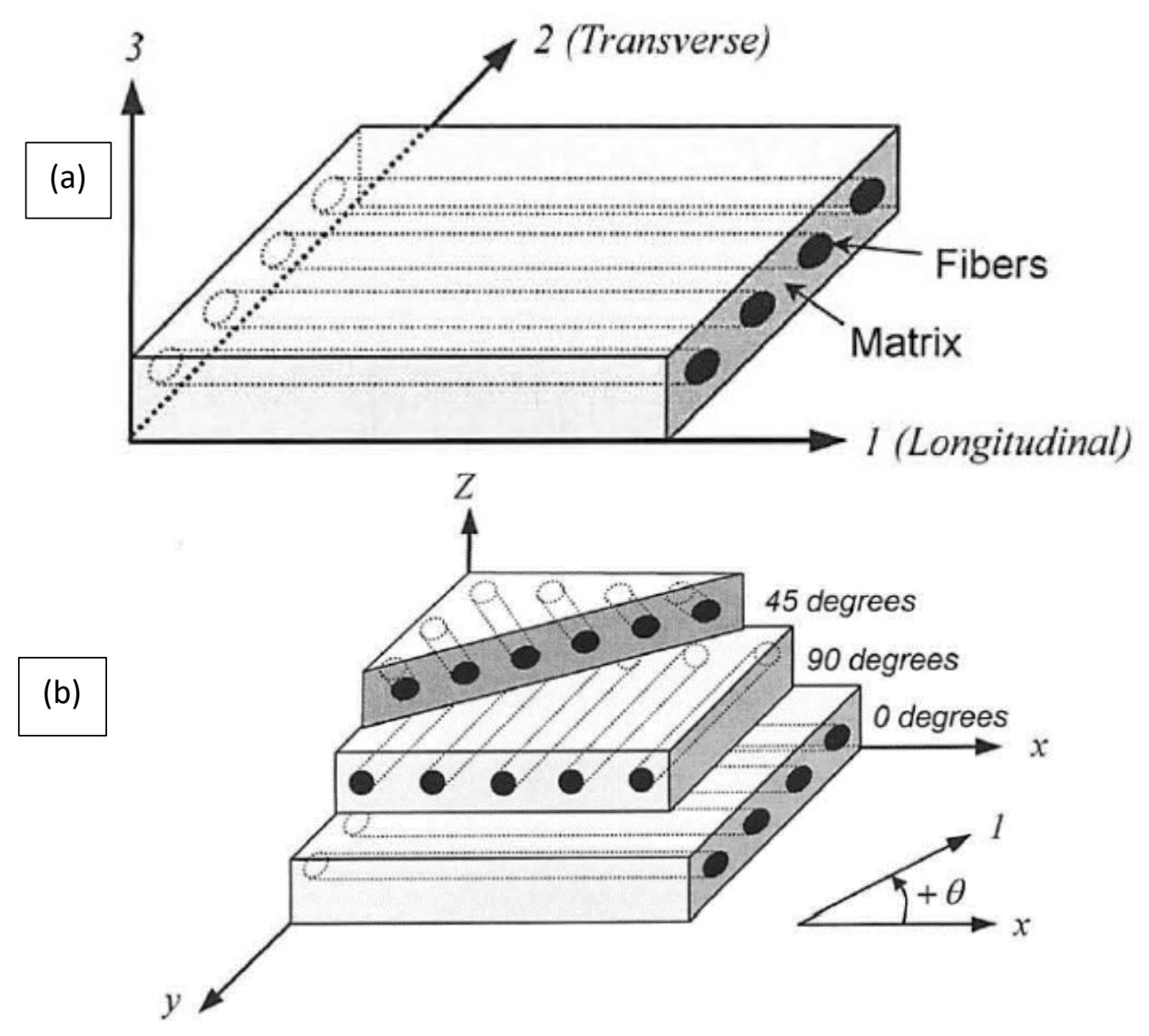

2.12: (a) Unidirectional lamina; (b) multidirectional laminate (Fam, 2000)

\subsubsection{Glass Fibre-Reinforced Polymer (GFRP)}

Glass fibre-reinforced polymer (GFRP) is the most commonly used fibre type for composite reinforcement. GFRP's are a processed form of glass, which is composed of several oxides (e.g., silica oxide) combined with other raw materials such as limestone, fluorspar, boric acid and clay. The final product made by drawing the melted oxides into filaments ranging from $3 \mu \mathrm{m}$ to $24 \mu \mathrm{m}$.

\subsubsection{Carbon Fibre-Reinforced Polymer (CFRP)}

Carbon Fibre-Reinforced Polymer (CFRP) or "graphite" fibres are a type of high-performance fibre. This type of fibre is manufactured by controlled pyrolysis and crystallization of organic 
precursors at temperatures above $2000^{\circ} \mathrm{C}$. In this process, carbon crystallites are produced and orientated along the fibre length. Carbon fibres are the most expensive, costing approximately 5 to 7 times the cost of glass fibres. They are lightweight and have a much higher stiffness and strength durability as compared with the other commonly used fibre types. They also have a very high resistance to dynamic loading, high fatigue and creep resistance, and low thermal expansion.

\subsection{Past Studies on FRP Piles}

Most past studies on FRP piles are focused on the load transfer and flexural response of FRP piles under static loading. Therefore, there are limited studies to understand the seismic behaviour of FRP piles. This section provides a summary of these studies on FRP materials over the past two decades.

Iskander et al. (2001) developed a theoretical parametric study on the effect of various pile properties and soil deposit conditions on the driveability of FRP piles. Results of these analyses revealed that composite piles may well be driven to reasonable capacities for load-bearing piles. Helmi et al. (2006) studied the influence of driving forces and high-cycle fatigue on flexural performance of concrete-filled glass/carbon-FRP piles (GFRP and CFFT) and reported that driving forces may have minor influence on the flexural strength of concrete filled fiber reinforced polymer tube (CFFT) piles. Correspondingly, CFFT piles were reported to have higher deflections compared to pre-stressed concrete piles. Additionally, a full scale CFFT pile was tested under reversed cyclic bending at $60 \%$ of ultimate moment and suggested that the service moment is about $20 \%$ to $30 \%$ of the ultimate moment to reach at least 1 million cycles.

Sakr et al. (2007) suggested fibre-reinforced polymers (FRP)-concrete composites as a substitute to increase deep foundation's durability compared to traditional pile materials such as 
steel, concrete and timber. In this investigation, FRP tubes were fabricated and filled with concrete materials. Driving tests were performed to explore the driving performance of FRP-concrete pile by considering the pile material and its geometry. Furthermore, driveability of FRP-concrete and traditional pile materials was analysed by applying the wave equation analysis using a computer program named PDPWAVE. Results showed that the driveability of FRP-concrete composite piles is similar to pre-stressed concrete and steel piles. Nevertheless, FRP tubes needed larger driving energy and it may not be recommended to use driving method for FRP tubes since it consists of lower flexural resistance; therefore, the risk of buckling is higher.

Giraldo and Rayhani (2014) performed lateral loading tests in the field and evaluated the lateral capacity of small model piles using traditional and modified FRP tubes including typical open-ended steel and closed end steel pipe, and Hollow FRP piles (glass and carbon). The result shows that Hollow FRPs can present the same advantages as concrete filled FRP piles due to its resistance to degradation and corrosion and similarly offering certain structural and geotechnical properties to even act as load bearing frictional piles.

Bryden et al. (2015) stated that FRP materials can be a great alternative to replace traditional piles specifically steel piles as the cost of steel and its fabrication is increasing proportionally and deterioration of steel material is another unsolved issue. Therefore, it is an opportunity for geotechnical engineering to introduce this material commercially in applications such as offshore structures, penstock for hydroelectric power plants, etc. Lately, several large FRP pipes were installed in Eastern Canada and the United States to substitute old timber-stave pipes to transport water into turbines of hydroelectric plants. In this paper, the researchers investigated the flexibility which is determined based on their effective stiffness. 
Fam et al. (2016) studied the durability of GFRP piles in bridge deck applications and the investigation was conducted using a series of chemical testing to understand the behaviour and change in properties of GFRP in salt solution as it ages. Results showed that after 224 days, the tensile strength retentions of a flat plate with T-shaped ribs (R-GFRP) and a corrugated plate were similar. A $14 \%$ reduction in tensile strength was observed when the temperature increased from 23 to $55^{\circ} \mathrm{C}$. Additionally, the observed reductions were evaluated using micrographs which showed surface cracks and splits between the fibres and the matrix. Results also showed that the polymer matrix was not fully degraded by the hydrolysis since this did not cause any significant variations in the glass transition temperature after exposure.

Murugan et al. (2017) conducted a series of cyclic lateral loading tests on glass and carbon fibre-reinforced polymers (GFRP and CFRP) strengthened reinforced concrete piles. This study focuses on the change in deflection, stiffness degradation, and energy dissipation profile of GFRP and CFRP strengthening reinforced concrete piles. The results from the field tests and the finiteelement model in this study showed that the CFRP-confined pile with fibre orientation along the length of the pile demonstrates higher load carrying capacity whereas the unconfined concrete pile reached lower capacity. Moreover, both CFRP and GFRP piles with fibre orientation through the pile shaft showed higher strength compared to those with fibres aligned around the circumference. Similarly, the unidirectional GFRP orientation along the length presented a greater load-carrying capacity than CFRP with fibre orientation through circumference.

Fam et al. (2003) used a series of concrete filled FRP tubes as a substitute to precast piles for Route 40 highway bridge over Nottaway River in Virginia. The piles consisted of $625 \mathrm{~mm}$ diameter concrete-filled GFRP tubes with wall thickness of $5.3 \mathrm{~mm}$ (Figure 2.13). Laboratory tests 
were employed to estimate the bending configuration and field testing was performed on this composite pile and conventional concrete pile to compare their behaviour under axial and lateral loading. However, the research team recommended further examination for aspects including piles' behaviour under combined loading, bonding improvement, flexural stiffness (prestressing), and behaviour under seismic loading and life cycle.

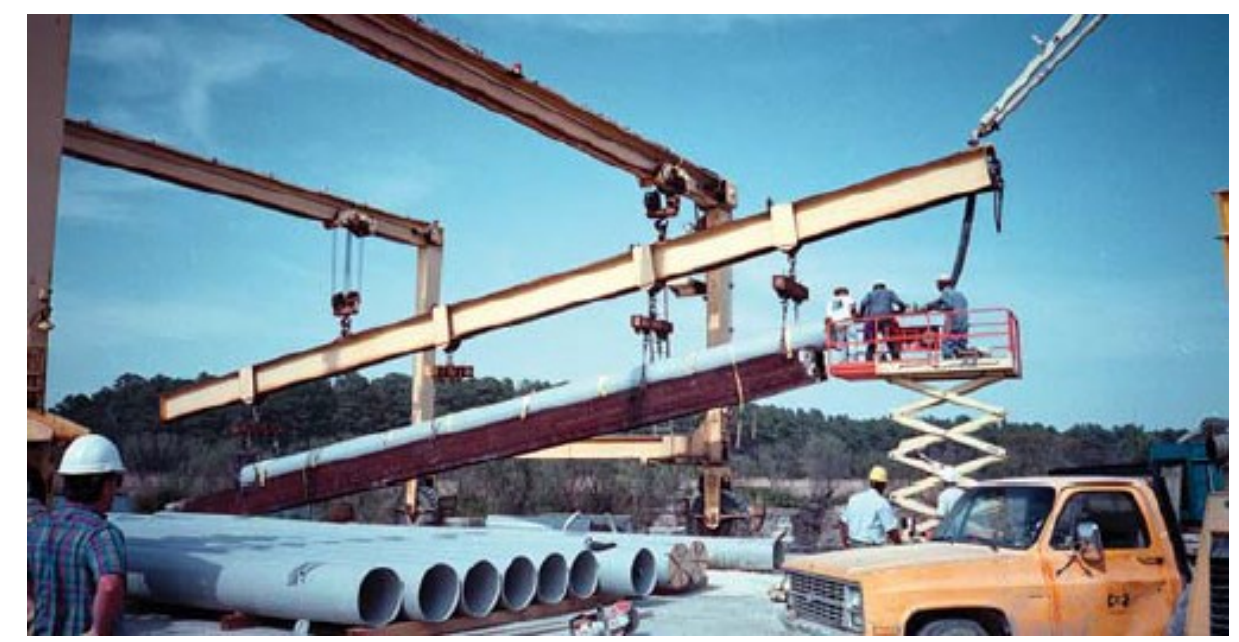

Figure 2.13: Fabrication and handling of precast composite piles (Fam et al, 2003)

Zaghi et al., (2012) investigated the seismic performance of a concrete filled fiber reinforced polymer (FRP) tube (CFFT) bridge column using shake table testing and nonlinear dynamic analyses of a one-fifth scale two-column bridge pier that also incorporated a conventional (RC) column (Figure 2.14). The FRP tube in the CFFT column was a prefabricated composite pipe with glass fibers aligned in $\pm 55^{\circ}$ with respect to the tube axis to deliver both hoop and longitudinal strengths. The result of shake table experiment demonstrated that CFFT columns can perform as a ductile, reliable column in bridges located in seismically active regions. No visible damage was detected in the CFFT column under drift ratios as high as 7\%. The tensile rupture of the FRP tube occurred during the last shake table run with 1.9 times the motion obtained during the Northridge 
1994 earthquake and under a drift ratio of 8.4\%. Between the two columns, the RC column damped a larger portion of energy compared to the CFFT column; however, it experienced severe spalling of the cover concrete and extensive shear-flexure cracking.

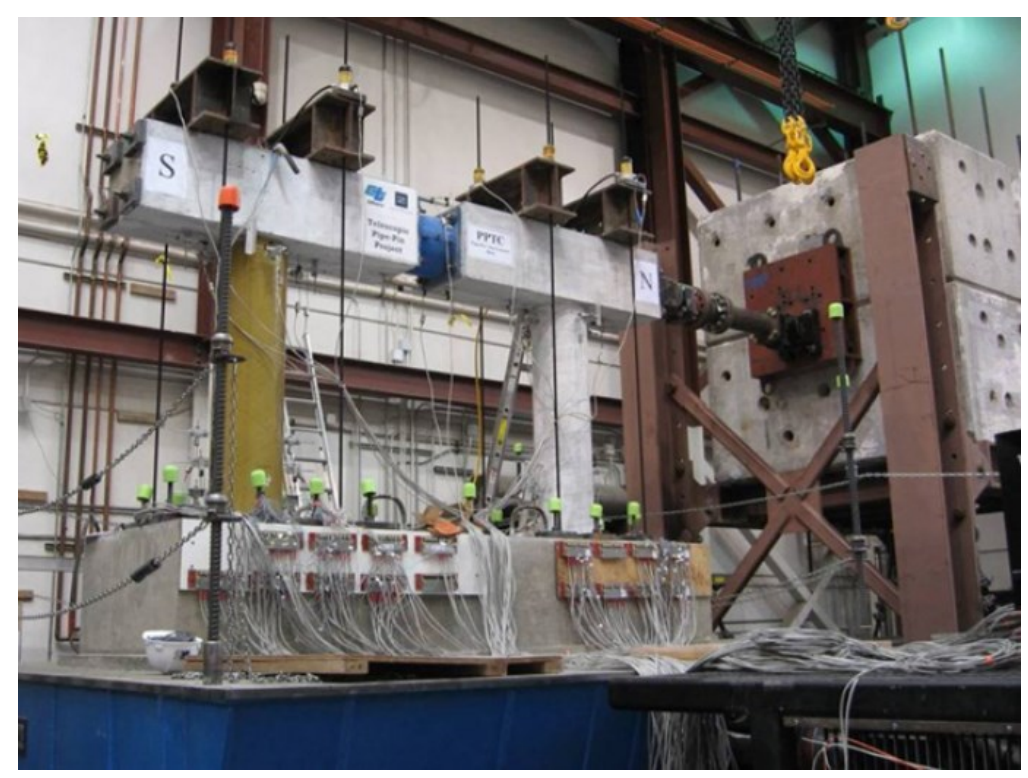

Figure 2.14: Two-column pier shake table setup (Zaghi et al., 2012)

\subsection{Previous Experimental Studies on Seismic Soil-Pile Interaction}

Several field and laboratory experiments have been performed to investigate the seismic SSI for pile foundations in different site conditions. Variety of techniques were introduced and used to understand the behaviour of soil-pile individually and in groups with all range of dimensions. Field tests have shown number of advantages over the laboratory tests since it can provide precise soil and structure stress conditions, while laboratory tests are more feasible and flexible to comprehend parametric evaluations in a meticulous environment. Both field and laboratory experiments are valuable and there are correlations among both specifically when the collected data is missing the SSI response. This section provides a survey of soil-structure experimental 
research focusing on deep foundation to evaluate previous practises and the objective of further research requirements.

\subsubsection{Field Tests}

Pile load testing programs in the field can provide discrete advantage of employing certain soil condition, practical pile, and consequently soil-pile stress condition in actual field. However, the form of loading or loading control is typically limited to the top of the pile which means that the effect of inertial interaction is only considered, and kinematic interaction is ignored. This section describes some of past studies on single and group piles under lateral load and dynamic load tests. The American Society for Testing Materials provides a standard procedure for conducting lateral pile load tests under specification ASTM D-3966 (ASTM, 2011). Schematic of a typical lateral load tests is shown in Figure 2.15.

Matlock (1962) performed a number of static, cyclic, and post-cyclic lateral loading tests on steel pipe piles with diameter of $320 \mathrm{~mm}$ embedded at about $13 \mathrm{~m}$ depth in different soft clay sites. The undrained shear strength of the soft clay was measured at a range of $14 \mathrm{kPa}$ to $38 \mathrm{kPa}$ (in upper deposit). Results showed a non-linear soil-pile response at upper layers and, eventually, it reached linear response at higher depths. Reese et al. (1975) drove single steel pipe piles with diameters of $150 \mathrm{~mm}$ and $600 \mathrm{~mm}$ into a stiff, overconsolidated clay deposit near Austin, Texas. The site was pre-excavated $1.0 \mathrm{~m}$, where water was held at the surface level to simulate the ocean floor condition. Unconfined compressive strengths of the soil were measured at 190 to $380 \mathrm{kPa}$ in the upper $6.0 \mathrm{~m}$. A higher degree of cyclic degradation in soil resistance was observed in these tests which could be due to the impounded water and possible gap (water wash) at pile-soil interface that occurred during each cycle of loading and led to degrading resistance. It is essential 
to evaluate the period of cyclic loading which was in excess of 15 seconds as the noticed water wash effect could be predictable to differ with loading rate.

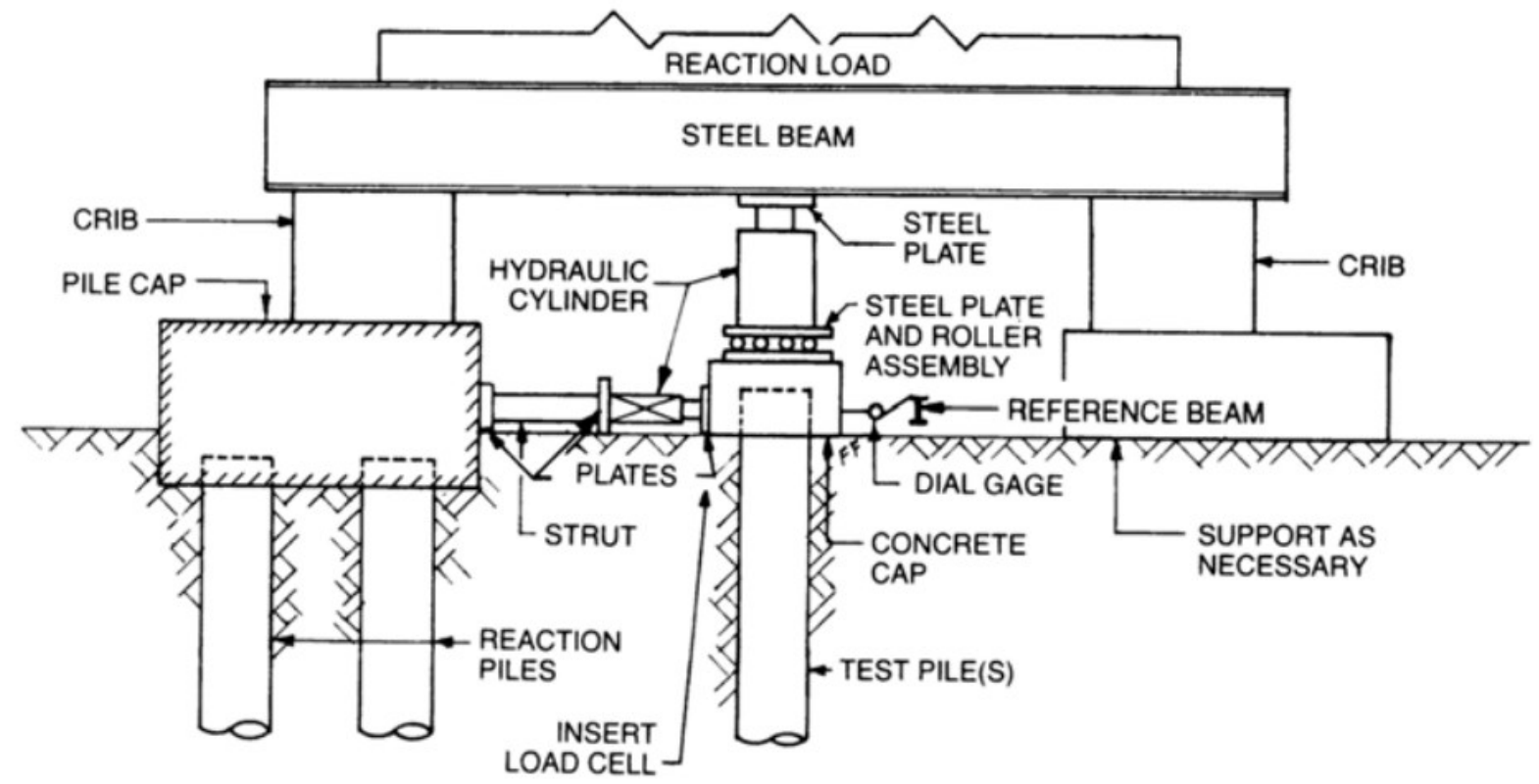

Figure 2.15: Lateral pile load test setup for combined lateral and axial compressive loads (ASTM D3966, 2011)

Luco et al. (1988) conducted field tests on a full-scale 9-story building. The purpose of the tests was to evaluate simple impedance function models that may duplicate the foundation impedance functions. The original hypothesis did not illustrate any substantial variances between the outcome of flexible and rigid foundations. Although the theoretical anticipations for rocking were rather accurate, the results of the tests did not show any change. Several dynamic tests were performed to estimate the pile stiffness on single or group piles using prototype or model scale piles in field. In these tests, a mass was fixed to the pile head to accentuate the resonant response and damping characteristics of the pile. 
ASTM-D4945-08 (as shown in Figure 2.16) introduces a standard dynamic load testing method that requires instrumentation of the pile by accelerometers and strain transducers. This test method involves measuring the pile elastic response to the force of the impact hammer during driving. The pile driving hammer drops and produces compressive waves in the pile and pile-soil interface. Any change in these waves is recorded using accelerometers and strain transducers measuring the pile response, and a pile dynamic analyser (PDA) device is used to compute the pile resistance based on a numerical integration of a dynamic wave equation. This test can provide the amount of resistance distributed between the shaft and toe of the pile.

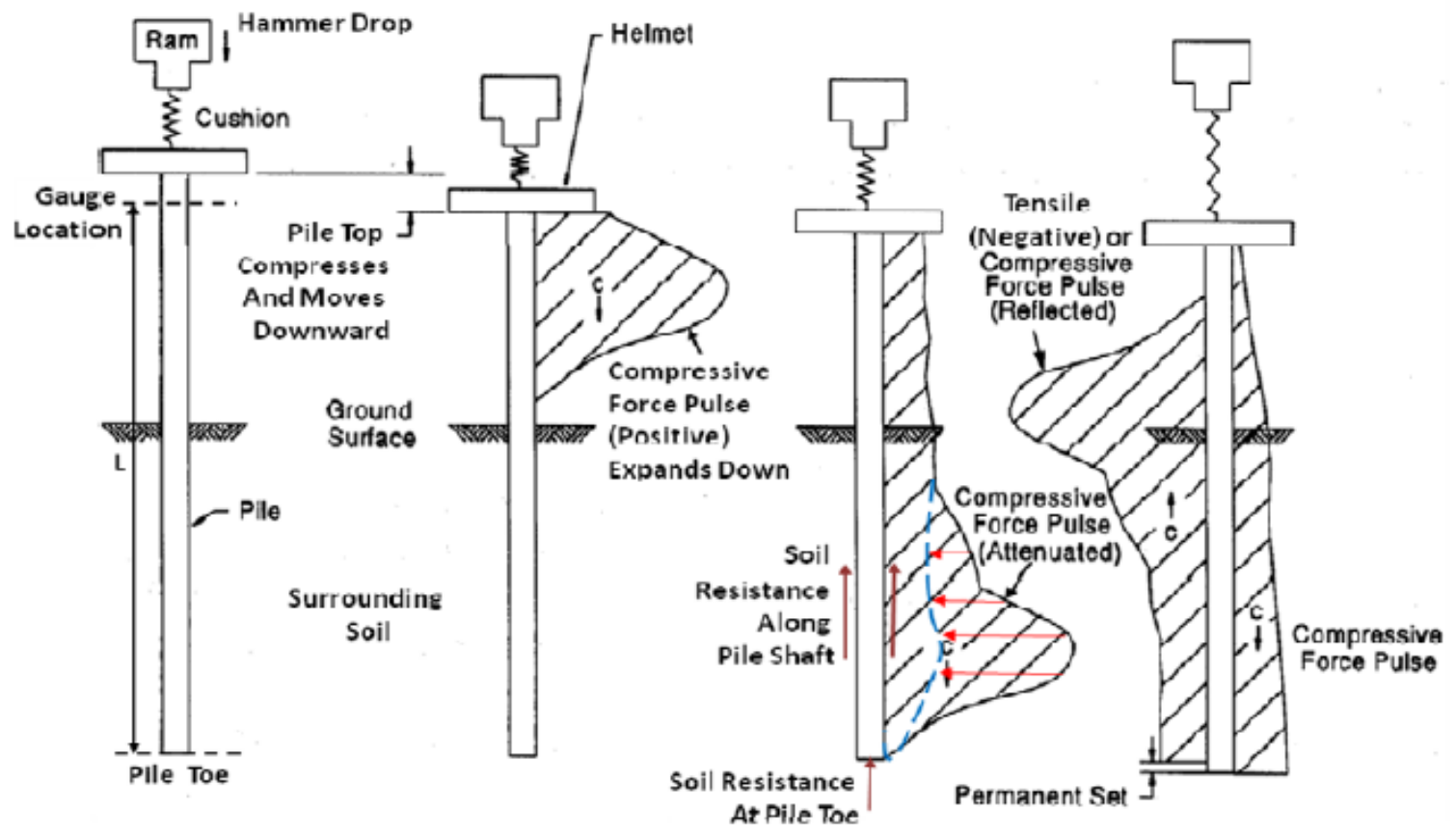

Figure 2.16: Wave propagation in piles during dynamic pile load test (Ng et al., 2011)

The PDA associated tests are classified into three types called ringdown, impact, and forced vibration tests. Ringdown tests consist of quickly releasing the pile from initial lateral displacement and determining the resultant free vibrations of the pile as the pile tries to reach its 
original position. Consequently, pile stiffness and damping values can be estimated from the free vibrations of the pile. Impact tests are lower strain type of a ringdown test, where a shock to the pile creates free vibrations in the pile. Forced vibration tests consist of eccentric mass shaker which is fixed on pile head and a spinner motor is installed to generate vibrations at the pile head. This is common method since the orientation can be adjusted and the direction of the vibration can be controlled (i.e., horizontally, vertically, and rocking vibrations) with different ranges of frequency and amplitudes. Scott et al. (1982) performed horizontal forced vibration and ringdown tests on an instrumented steel pipe pile driven into silty sand. The extensive field instrumentation monitored pile bending moments, pile head displacement and acceleration, pore pressures in the surrounding soil, and ground surface velocity in the free field.

Capatti et al (2018) performed dynamic tests on full-scale vertical injected and not injected micro-piles embedded in alluvial soils. The experimentation included ambient vibration and impact load tests to evaluate the dynamic behaviour of the soil-pile interaction system in small to medium strain ranges. In this study, the micro-piles were mounted with strain gauges along the shaft, and accelerometers and displacement transducers were installed at the head. Ambient vibration test showed to be a useful technique to understand and verify the dynamic properties of soil-micro-pile systems, while it may need complex post-processing techniques for data interpretation. The impact load test is flexible to setup and perform post process. Additionally, free vibration tests demonstrated to be comparatively convenient to conduct and record the dynamic non-linear behaviour of soil-micro-pile systems.

\subsubsection{Shaking Table Tests}

Field observation may not always reflect the soil behaviour during earthquakes because of limitations with the location and characteristics of an earthquake. On the other hand, several 
instruments and tools are required to establish a complete setup for SSI analysis on current structures. Moreover, there are very limited records of strong motion for the SSI systems at a failure state. Therefore, it is necessary to develop physical models in more controlled environment in order to perform seismic soil-structure testing. Centrifuge and shake table model tests can be efficiently used to examine complicated SSI phenomenon with desired loading conditions and conduct detailed parametric studies. Several researchers performed shaking table tests to understand key feature responses of scaled soil-foundation models.

\subsubsection{Earthquake Simulation using Shaking Table}

The nature and distribution of earthquake damage is strongly influenced by the response of soils to cyclic loading. This response is controlled in large part by the mechanical properties of the soil. Every region consists of its particular rock and soil properties and the distribution of these soil types can vary wildly within small areas. As a result, two locations within the same distance from an earthquake's epicenter can experience significantly different effects. One may experience over ten times the impact of another only due to geological variations known as local site conditions, which are based on two typical characteristics; primarily the softness of the soil and/or rock, and secondly, the total depth of the soil deposit above the bedrock. This indicates that characteristic of site condition can play essential role during an earthquake. Thus, any wave transition from stiff bedrock to soft deposits can cause to increase the amplitude which ends up to stronger shaking. Similarly, higher depth of soft soil deposits rested on bedrock can allow seismic wave to travel thru and develop greater amplification. These effects reveal that local site conditions can severely affect the outcome of amplitude, frequency content, and duration-of strong ground motion. Hence, the level of impact depends on the geometry and material properties of the subsurface materials, on site topography, and on the characteristics of the input motion. The nature of local site effects 
can be demonstrated in several ways: by theoretical ground response studies, by measurements of surface and subsurface motions at the same site, and by measurements of ground surface motions from sites with different subsurface conditions (Kramer, 1996).

For long time, model tests are suggested to duplicate the seismic environment, and designed to replicate the boundary conditions of a certain problem by implementing a smallerscale of physical model of a full-scale prototype foundation and/or structure to seismic loading. This approach is certainly used to evaluate the performance of a prototype or to develop parametric studies on various seismic challenges in order to identify critical phenomena and verify predictive theories. Model tests are globally conducted under the gravitational field of l-g known as Shaking table tests or high gravitational accelerations called centrifuge tests.

The shaking table test is one of the most widely used techniques to assess the seismic performance of foundation and structures made of various materials. Commonly, it is widely used for assessing linear/nonlinear and elastic/inelastic dynamic response of foundations and structures. In the early years of geotechnical earthquake engineering, virtually all physical model testing was performed on shaking tables. Shaking table research has offered valuable insight into liquefaction, post-earthquake settlement, foundation response, and lateral earth pressure problems. In shaking table tests, most researchers used scaled models as specimens. For example, Liu et al. (2016) conducted shaking table tests on a 1:30 scaled model with and without base isolation bearings to assess the seismic performance of an isolated museum structure in high earthquake intensity regions. Lu et al. (2007) performed 1:50 scaled high-rise building model on a shaking table to understand the seismic characteristics and failure mechanisms of the building. Graziotti et al. (2017) conducted a shaking table test on a two-story full-scale unreinforced masonry building to study its response, characteristics, damage mechanism, and evolution during the experimental 
phases. Several other researchers completed seismic studies of particular superstructure and specific foundation in variety of scales. Martinelli et al., (2009) presented the nonlinear dynamic response of a shaking table test for a full-scale seven-story reinforced concrete shear wall building, where four simulated earthquake records with increasing intensity were used as excitation. Saranik et al., (2012) conducted a shaking table test to investigate the inelastic behaviour of a two-story steel portal frame with bolted connections. Furthermore, it is not only used for structural dynamic

tests but also for geotechnical behaviour. Chen et al., (2013) conducted a series of shaking table tests on a plaster model of a three-story and three-span subway station to investigate the seismic failure characteristics of the structure on the liquefiable ground. Srilatha et al. (2013) studied the impact of frequency of base shaking on the dynamic response of unreinforced and reinforced soil slopes through a series of shaking table tests.

The above literature review suggests that the shaking table test is an essential tool to assess and verify the dynamic behaviour of foundations and structures. It is particularly essential for those foundations and structures that exceed the limits of the specification of design codes and standards.

\subsubsection{Classification of Shaking Table Models}

Model tests in geotechnical engineering offer the advantage of simulating complex systems under controlled conditions providing the opportunity of better understanding the fundamental mechanisms of these systems. Shaking table test is an experimental technique used in earthquake engineering to simulate ground motions. Since the emergence of shaking table tests in the $1920 \mathrm{~s}$, large numbers of earthquakes model tests have been performed. Shaking table tests have been considered as $1 \mathrm{~g}$ modelling, in which the gravity acceleration of the model and prototype are always the same. The geotechnical models cannot be directly mounted on shake table because of the requirements of confinement. To model the soil in shaking table tests, a container is required 
to hold the soil in place. In general, this container is called Soil Container, Soil Tank, Shear Box, Laminar/Container Box.

Soil containers can be classified into three major categorises, specifically, rigid, flexible, and laminar containers. Rigid containers are the simplest type and initial models consisting of no moving components. In this design, the shear stiffness of the wall is much higher than the stiffness of the soil layers within the container. As stated by Jakrapiyanun (2002), studying earth retaining structures such as retaining walls, bridge abutments, and quay walls appears to be conducted in rigid wall containers as the soil on one side of the earth retaining structure is lower than the other side. Accordingly, the soil on the shallower depth is less inhibited (Figure 2.17). The main disadvantage of the rigid containers is misleading the free field boundary conditions. This weakness occurs as a result of the rigid walls which prevent movement of the soil deposit and generation of excessive energy reflections from its boundaries. These impacts can be solved by manufacturing extremely large containers which is mainly not feasible. Another alternative solution can be energy absorbing layers such as foam or sponge attached on end-walls in the container which can provide reduction of wave/energy reflection, and reduction of lateral stiffness of its walls. Steedman and Zeng (1991) revealed that only one third of incident waves could be reflected by the absorbent boundaries. Gohl and Finn (1987) states that even with the help of absorbent boundaries to reduce reflection, those boundaries may still cause additional modelling variables such as stiffness and friction of the layers. Valsangkar et al. (1991) employed 25-mm thick Styrofoam (i.e., foam board) as the absorbing layers in their rigid container which were affixed to both end walls perpendicular to direction of shaking. 


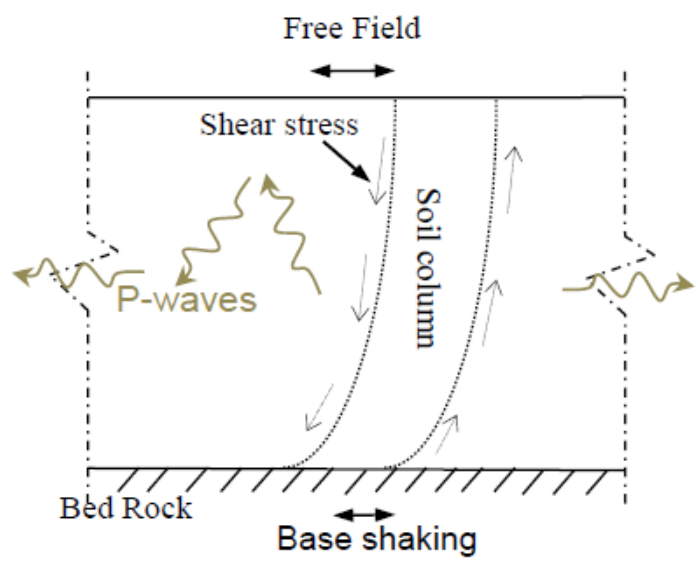

(a)

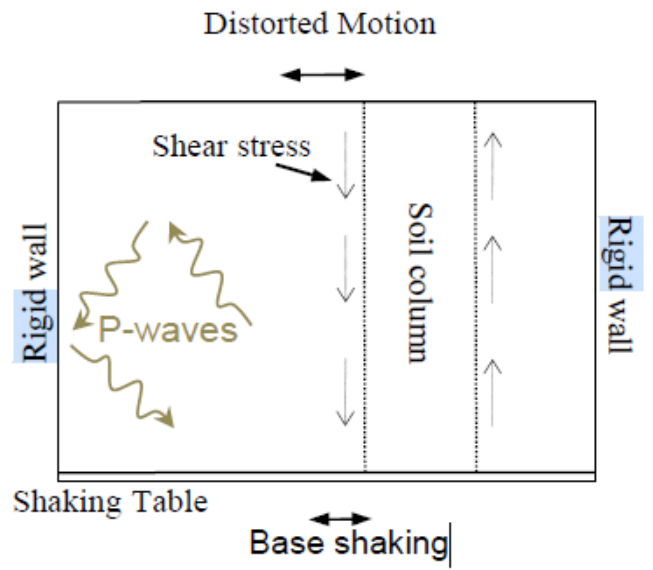

(b)

Figure 2.17: Comparison of (a) the free field ground motion, and (b) the simulated motion employing rigid soil container on shaking table

Flexible containers allow the soil to move in equivalent to the free field ground motion in comparison with rigid containers ( $\mathrm{Lu}$ et al., 2004; Moss et al., 2010). Moreover, reflection of outward propagating waves back into the model from the walls could be reduced effectively. Meymand (1998) conducted a series of shaking tests in flexible soil containers as shown in Figure 2.18. This container is cylindrically shaped which is $2440 \mathrm{~mm}$ diameter and $2130 \mathrm{~mm}$ in height. The top ring and the base plate were made of steel plates. The top ring was supported by four steel pipes to allow full translational and rotational freedom to the soil container. A rubber membrane was bolted to the top ring and the base plate with compression rings in between $50 \mathrm{~mm}$ wide bands which were dressed around the exterior wall of the membrane. This arrangement can deliver the lateral flexibility and radial stiffness (to avoid bulging). Furthermore, the soil container base plate was roughed by applying high friction epoxy coating with addition of angular crushed gravel pieces onto its surface, and the soil deposit was isolated before reaching the top ring. 


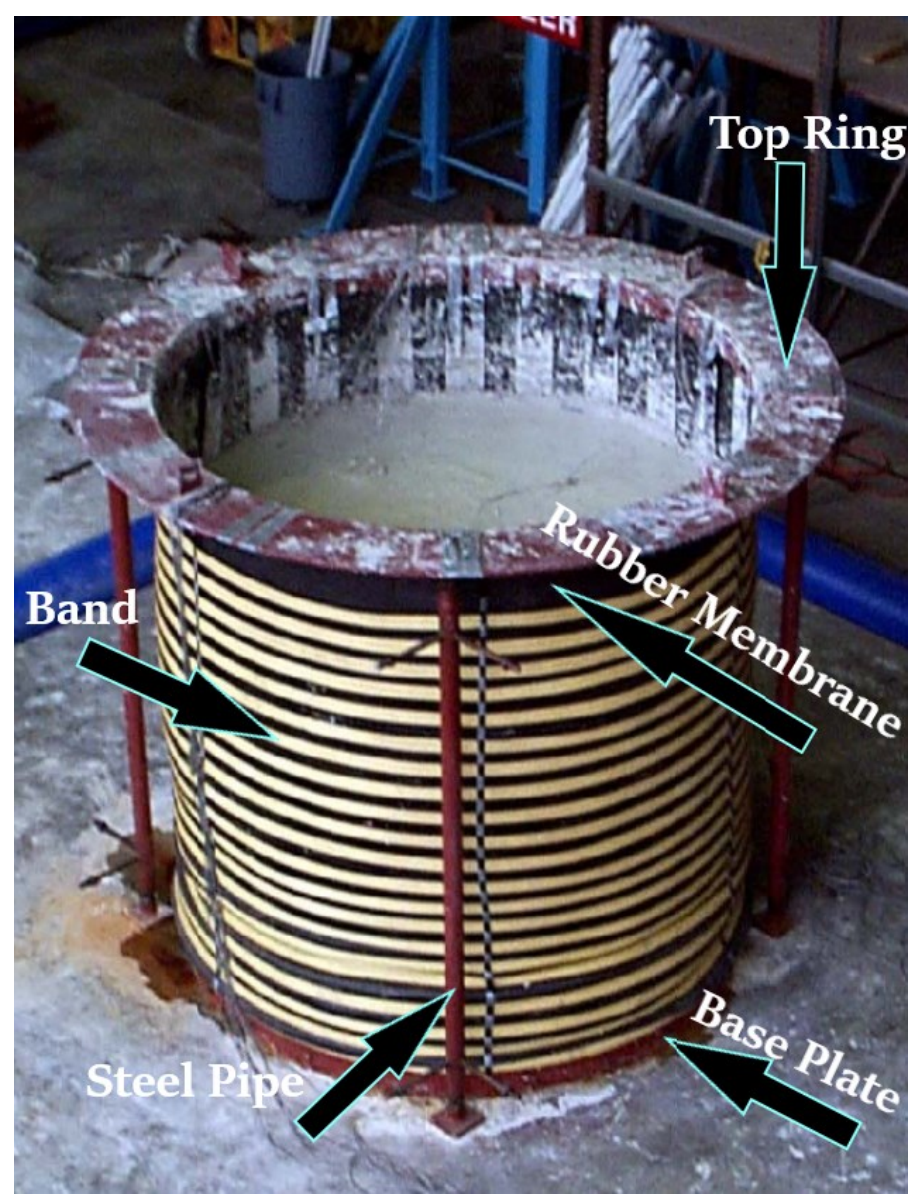

Figure 2.18: Flexible cylindrical soil container (Meymand, 1998)

Alternatively, Gazetas (1982) stated that laminar soil containers can realistically simulate the free field conditions in comparison with rigid and flexible containers. Several types of interlayer sliding systems for laminar container have been introduced over the past few decades such as commercial ball bearings (Ishimura et al., 1992), sliding systems such as Teflon (Chau et al., 2009), and elastic materials such as rubber (Taylor, 1997). The most common design consists of a stack of laminae supported individually by bearings (Figure 2.19). The design principle of a laminar box is to minimize the lateral stiffness of the container in order to ensure that the soil governs the response of the soil container system. 


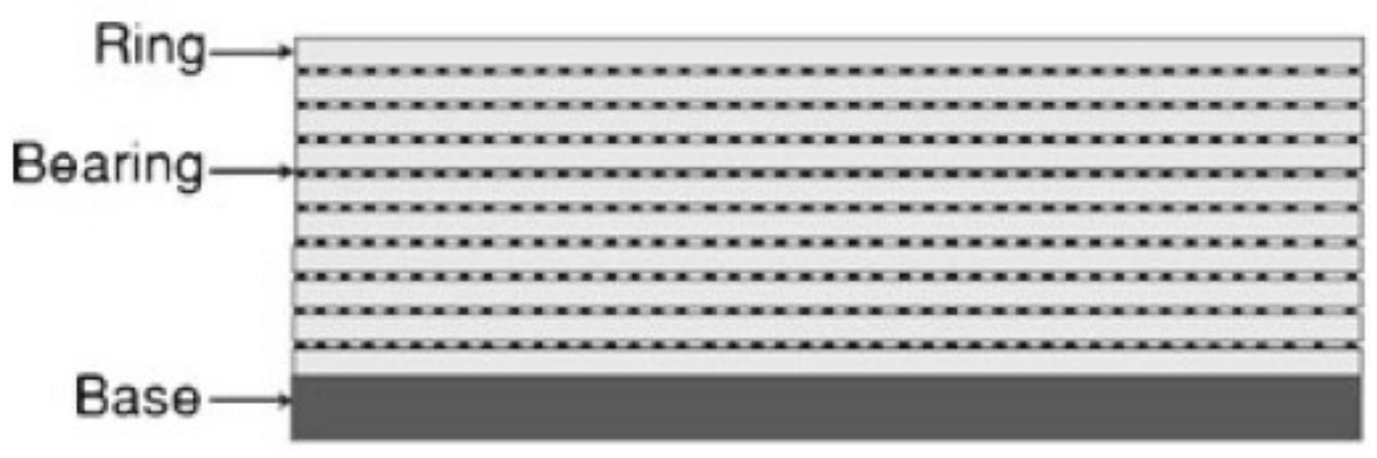

Figure 2.19: Schematic view of laminar container

Latest studies revealed that lateral movements of the laminar container in shaking table tests can be nearly identical to the free-field ground motion. Several researchers (e.g., Gazetas, 1982; Taylor et al., 1995; Pitilakis et al., 2008; Tang et al., 2009; Tsukamoto et al., 2012; Massimino and Maugeri, 2013) concluded that the laminar soil container is the highly reliable and efficient type of the soil container. Based on the closures by the previous researchers, the advantages of employing shaking tests in laminar containers are as follows:

- Durable and well-designed laminar soil containers can be reliable model to simulate the free-field conditions, since the lateral deformations applied in uniform pattern and very similar to the free-field movements,

- Lateral motion of the entire depth follows the sinusoidal shape which represents authentic conditions of the free-field ground motion,

- Latest experimental tests in shaking table are performed using the laminar soil container due to its accuracy in modelling the free-field ground motion.

According to the above-mentioned merits of using laminar soil containers over the other types of containers (flexible and rigid soil containers) and in order to perform rigorous and reliable experimental shaking table tests, a laminar soil container is employed in this study. 


\subsubsection{Previous Shaking Table Tests on Pile Foundations}

Shaking table tests in 1-g environment have also been used to investigate the response of model piles in wide range of soil conditions. Such model tests can offer a number of advantages in simulating pile-soil systems and provide opportunities to understand fundamental mechanism of soil-foundation interaction and validate analytical solutions. Gazetas (1982) reported that laminar soil containers can convincingly simulate the free field conditions in comparison with rigid and flexible containers. Many other researchers (e.g., Taylor et al., 1995; Pitilakis et al., 2008; Tang et al., 2009) concluded that the laminar soil container is the most suitable and efficient type of the soil container. They also found out that the lateral deformations in laminar soil containers are almost identical to the free field movement, thus, uniform lateral motion exists in each horizontal plane. Lateral motion of the entire depth in the soil model follows the sinusoidal shape which represents authentic conditions of the free field ground motion.

A series of the past shaking table studies was focused on performance of the soil inside the shake with no structural elements or components including Taylor (1997), Prasad et al. (2004), and Lee et al. (2012). The purpose of these tests was to evaluate the behaviour of the soil, typically local soils, in order to comprehend the impact of earthquake loads on soil amplification and damping. Alternatively, other researchers moved some steps forward and developed variety of foundation systems to understand the dynamic response of foundations in different soil mediums (Meymand, 1998; Lu et al., 2004; Chau et al., 2009).

Kubo (1969) was one of the first researchers who performed shaking table testing on model piles. Results highlighted the impact of kinematic interaction within the soil-pile system. The bending moment profiles of the model piles and their deflection along the shaft were shown to be consistent with selected prototype behaviour. Nomura et al. (1991) performed shaking table tests 
to verify the rigidity of different pile materials. Additionally, free-field response analysis was done on a horizontal subgrade reaction pile model to simulate the experimental results. From the tests, it was concluded that the dynamic response of the pile-structure system is significantly affected by the response of the soil prior to liquefaction as well as the liquefaction. Although, this research focused on the pile rigidity but a number of other effective parameters such as relative density of the soil, soil placement and layering, pile spacing (boundary effect) and installation technique, and input motions were found to affect the soil-pile behaviour.

Meymand (1998) performed a set of scale model shaking table tests on model piles in synthetic clay to offer perception into seismic soil-pile-structure interaction (SPSI) and create data to regulate innovative SPSI analysis developed at U.C. Berkeley. The flexible soil container was cylindrically shaped with a $2400 \mathrm{~mm}$ in diameter and approximately $2100 \mathrm{~mm}$ in height. The top section and the bottom plate were made of steel. The top section was supported by four steel pipes to provide adequate translational and rotational movement. A rubber membrane was fastened to the top ring and the base plate. Therefore, lateral flexibility and proper stiffness were reached by the rubber membrane and bands that were aligned around the exterior of the rubber membrane (Figure 2.20). The model piles were driven into the soil in single or group configurations through a $460 \mathrm{~mm}$ tall template to ensure location and verticality. The result of the shaking experiment demonstrated that single piles were seen to respond with components of inertial and kinematic interaction, with the inertial components producing upper bound bending moments. The response of pile groups was highly frequency dependent, which may question the applicability of applying pseudo-static analyses to such problems. Pile cap and free field motion variations showed wave scattering effects and the necessity of developing modified foundation input motions for sub- 
structuring analyses. Moderate effects of pile cap embedment were observed, particularly in contributing to pile group rocking stiffness.

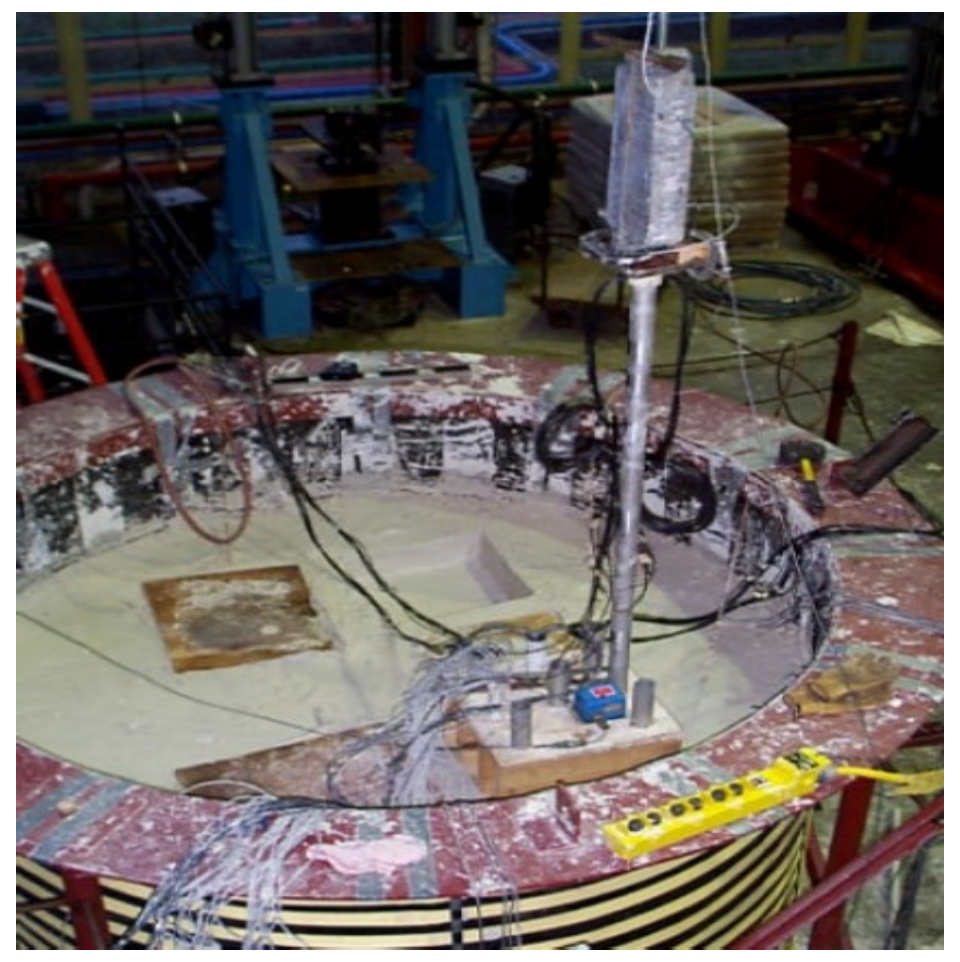

Figure 2.20: Model pile installation in shake table test (Meymand, 1998)

Hokmabadi et al. (2014) investigated the impacts of seismic soil-pile-structure interaction (SSPSI) on the dynamic response of buildings with different heights by performing a set of laminar shaking table tests on 5 to 15 -story small model structures (Figure 2.21). A fixed-base structure and a structure supported by an end-bearing pile foundation in soft soil were designed and tested under specific local input motions. Based on the experimental results, the SSPSI amplified the foundation input motions and induced lateral deflections in the model piles, which caused drifts of the structures supported by end-bearing piles compared to the fixed-base structure. It also impacted the rocking component of the structure which has critical role in the development of the lateral deflection of superstructures and possible collapse the structure. 


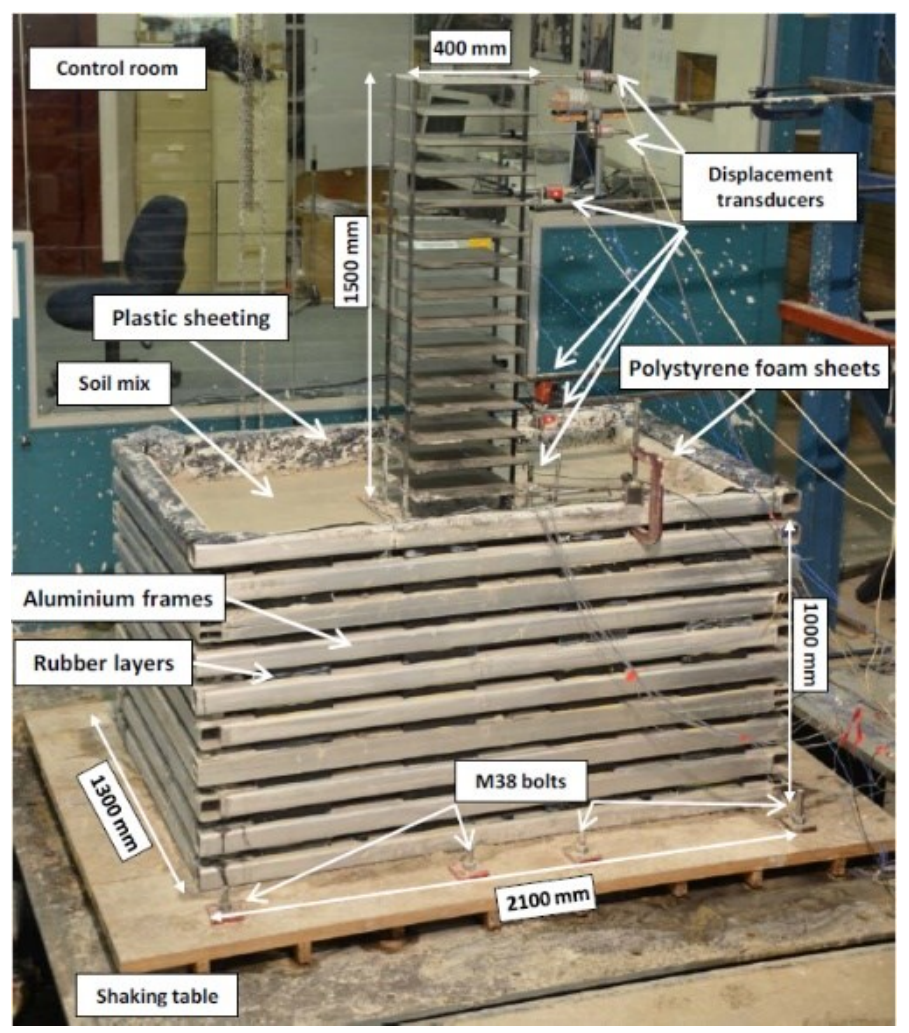

Figure 2.21: Example of a shaking table tests for the multi-story structure with pile foundation (Hokmabadi, 2014)

Taha (2014) used geosynthetics materials to enhance the lateral performance of pile foundations. This research conducted series of static tests and small-scale shaking tests to comprehend the soil-structure interaction behaviours of geosynthetics-reinforced pile foundation systems in a 1-g environment. The two different soil models included: a layer of synthetic clay underlain by a sand layer, and a layer of synthetic clay sandwiched between a sand layer and an aggregate layer (Figure 2.22). The results from seismic tests revealed that the lateral seismic response can be reduced by $50 \%$ by using geosynthetic reinforcement.

Unsever et al. (2017) performed a set of shaking table tests on frictional 3-pile piled raft and 3-pile pile group models embedded in saturated sand at 1-g environment. A sinusoidal wave motion was applied at a frequency of $20 \mathrm{~Hz}$. Results illustrated that the piled raft with ground contact can experience less settlement compared to the pile group even at liquified condition. Also, 
it was observed that piled raft may have reduction in horizontal resistance due to the loss of bearing capacity if liquefaction reaches the foundation level.

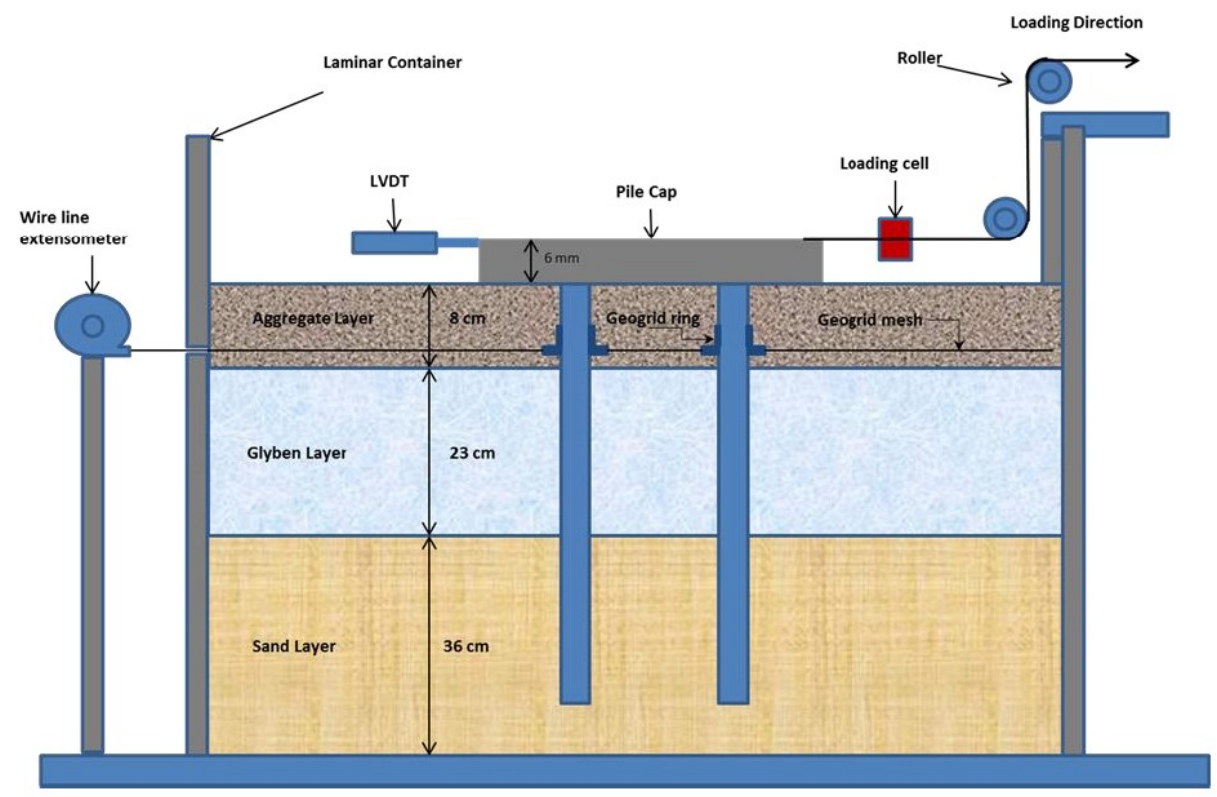

Figure 2.22: Schematic diagram of the shaking test setup (Taha, 2014)

Bao et al., (2012) evaluated the seismic enhancement effect of aluminium group-pile foundation by designing a small model test on a full system with a superstructure and aluminium group pile foundation embedded in sand. The shaking table test device is $120 \mathrm{~cm}$ in width and 160 $\mathrm{cm}$ in length (Figure 2.23). The result showed that the enhancement effect to reduce the deformation of an existing group-pile foundation and the bending moment of the piles can be accomplished with the partial ground improvement method. 


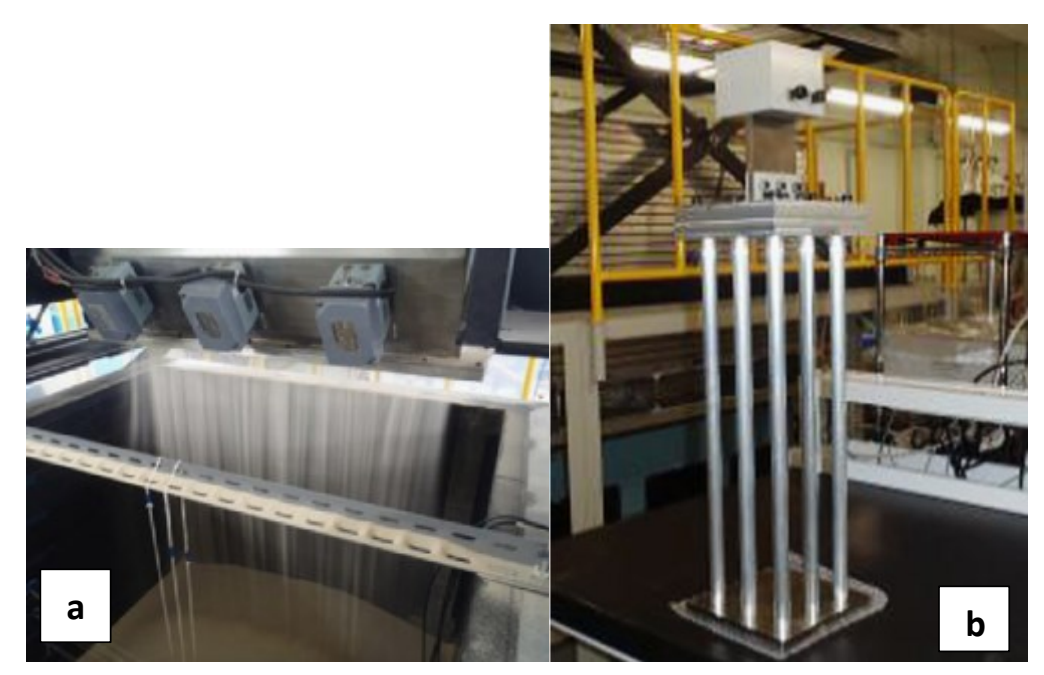

Figure 2.23: (a) Model group-pile foundation and superstructure.

(b) Sand dropping device lay on an up-down movable frame (Bao et al., 2012)

Yang et al. (2020) conducted a series of large-scale shaking table tests of tall buildings with nonlinear viscous dampers on soft soils supported on 3-by-3 pile group foundations to comprehend the influence of the SPSI on the seismic behaviour of the total system and impacts of using the viscous dampers (Figure 2.24). These tests were divided into two different models including a fixed-base structure with viscous dampers, which ignores the mechanism of soil-structure interaction (SSI), and another model with a structure supported on 3-by-3 pile within a shear laminar soil container. The experimental results illustrated that SPSI system has longer natural periods and the frequencies decrease more lightly than the fixed-base structure after the tests. Furthermore, the impact of SPSI on the frequencies are rather superior compare to the damping ratio. Accordingly, disregarding the SSI effects may result in impractical results of the seismic responses of the superstructure and overstate the performance of the nonlinear viscous dampers. 


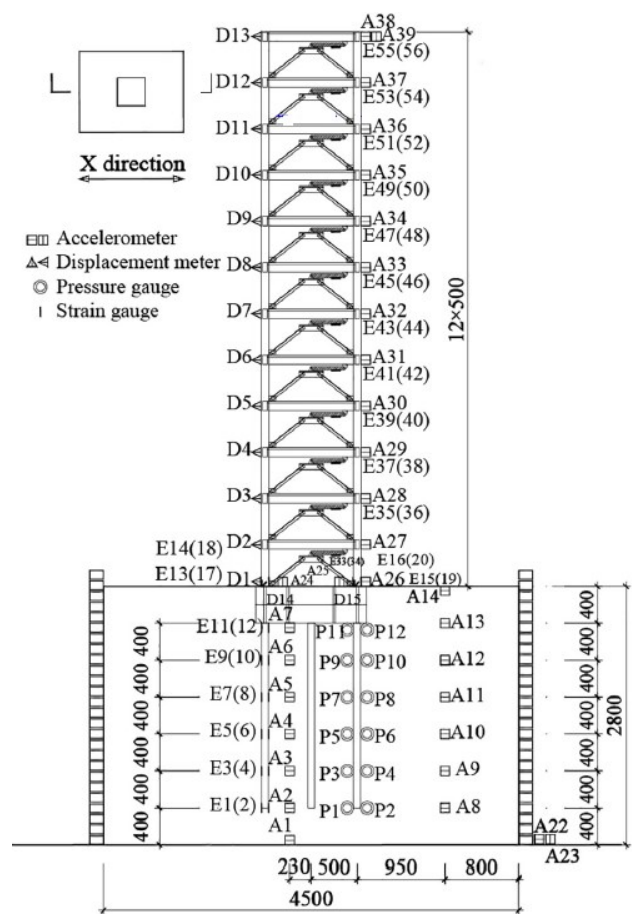

Figure 2.24: Experimental setup for performing shaking table tests 
Table 2.2: Summary of past shaking table tests using various types of soil containers

\begin{tabular}{|c|c|c|}
\hline Shaking Table Container Type & References & Remarks \\
\hline Rigid & $\begin{array}{l}\text { Gohl and Finn (1987) } \\
\text { Yan and Byrne (1989) } \\
\text { Valsangkar et al. (1991) } \\
\text { Zen et al. (1992) } \\
\text { Sato et al. (1995) } \\
\text { Lin and Wang (2006) } \\
\text { Bathurst et al. (2007) } \\
\text { Ha et al. (2011) } \\
\text { Anastasopoulos et al. (2012) }\end{array}$ & $\begin{array}{l}\text { Foundation type: conventional pile materials, } \\
\text { hollow aluminium piles, aluminium footing. } \\
\text { Soil medium: Dry Ottawa sand, } \\
\text { saturated sand, saturated sand mixed with } \\
\text { treated soil. }\end{array}$ \\
\hline Flexible & $\begin{array}{l}\text { Kanatani et al. (1995) } \\
\text { Stanton et al. (1998) } \\
\text { Meymand (1998) } \\
\text { Maugeri et al. (2000) } \\
\text { Lu et al. (2004) } \\
\text { Moss et al. (2010) }\end{array}$ & $\begin{array}{l}\text { Foundation type: shallow and deep } \\
\text { foundations such steel piles, and aluminium } \\
\text { piles. }\end{array}$ \\
\hline Laminar & $\begin{array}{l}\text { Ishimura et al. (1992) } \\
\text { Taylor et al. (1995) } \\
\text { Tao et al. (1998) } \\
\text { Jakrapiyanun (2002) } \\
\text { Prasad et al. (2004) } \\
\text { Pitilakis et al. (2008) } \\
\text { Chau et al. (2009) } \\
\text { Tang et al. (2009) } \\
\text { Turan et al. (2009) } \\
\text { Chen et al. (2010) } \\
\text { Lee et al. (2012) } \\
\text { Tsukamoto et al. (2012) } \\
\text { Massimino and Maugeri (2013) } \\
\text { Hokmabadi et al. (2014) } \\
\text { Yang et al. (2020) } \\
\text { Wu et al. (2020) } \\
\text { Chiou et al. (2021) }\end{array}$ & $\begin{array}{l}\text { Foundation type: shallow foundation. single } \\
\text { or group piles in different arrangements- } \\
\text { conventional piles (e.g., concrete, steel, } \\
\text { aluminium, polypropylene). } \\
\text { Soil medium: Dry sand, loose sand, medium } \\
\text { dense sand, and saturated sand, synthetic } \\
\text { clay, glyben clay. }\end{array}$ \\
\hline
\end{tabular}

\subsection{Scaling Factors for Shaking Table Tests}

Reduced scale model tests offer the advantage of studying the response of soil-pile-structure systems in a controlled environment. Proper modification and scaled model tests are also advantageous in seismic studies as they are able to provide more feasible and realistic information about ground amplification, pore water pressure variations, non-linear soil behaviour and soil 
structure interaction. Rocha (1957) pioneered developing scale model similitude relationships for soil mechanics problems. The stress-strain behaviour of soils was scaled assuming that they are linearly proportional in models and prototypes. Rocha's linear scaling concept was set to account for the changes in the stress system present in a 1-g environment. Iai (1989) derived similitude relationships assuming that the constitutive stress-strain relationship was independent of the confining stress if a proper scaling factor is used. He derived his similitude relations based on the geometric and density scaling factors. Consequently, the use of scaled models in engineering provides the advantage of simulating complex systems under controlled conditions. For such applications, it is essential to have a set of scaling relations describing the experimental model and predicted prototype behaviours (Meymand, 1998). Scale models can be referred to geometric, kinematic, or dynamic similarities to the prototype (Langhaar, 1951; Sulaeman, 2010). Geometric similarity states a model and a prototype with homologous physical dimensions. Kinematic similarity indicates a model and a prototype with homologous particles at homologous points at homologous times. Dynamic similarity defines a condition where homologous parts of the model and prototype experience homologous net forces. Scale models meet the requirements of similitude to the prototype to differing degrees, and researchers may apply nomenclature such as "true", "adequate", or "distorted" to the model (Moncarz and Krawinkler, 1981). A satisfactory model suitably scales the primary features of the problem, with secondary influences allowed to deviate while the prediction equation is not significantly affected. Distorted models refer to those cases in which deviation from similitude requirements distorts the prediction equation, or where compensating distortions in other dimensionless products are introduced to preserve the prediction equation. 
Moncarz and Krawinkler (1981) explained that in 1-g scale modelling, where $\rho$ is density, $E$ is modulus of elasticity, $a$ is acceleration, and $g$ is gravitational acceleration, the dimensionless product $a / g$ (i.e., Froude's number) must be kept equal to unity indicating that the ratio of model to prototype specific stiffness $(E / \rho)$ is equal to the geometric scaling factor $\lambda$. This is known as "Cauchy condition" which can also be specified in terms of shear wave velocity as follows:

$$
\frac{\left(v_{s}\right)_{p}}{\left(v_{s}\right)_{m}}=\sqrt{\lambda}
$$

where, subscripts $p$ and $m$ stand for prototype and model, respectively; $v_{s}$ is shear wave velocity. This relationship satisfies the Cauchy condition which is a required condition for simultaneous replication of restoring forces, inertial forces, and gravitational forces in a dynamic system (Moncarz and Krawinkler, 1981). Iai (1989) derived a comprehensive set of scaling relations for a soil-structure system under the dynamic loading and defined the entire problem in terms of geometric, density, and strain scaling factors. This technique relates the geometric $(\lambda)$ and density $\left(\lambda_{p}\right)$ scaling factors, and then derives the strain scaling factor $\left(\lambda_{\varepsilon}\right)$ from shear wave velocity tests on both the model and prototype soil, as follows:

$$
\lambda_{\varepsilon}=\left[\frac{\lambda}{\left(\frac{\left.v_{s}\right) p}{\left(v_{s}\right) m}\right)^{2}}\right]
$$

where, $\left(v_{s}\right)_{p}$ is shear wave velocity of prototype soil and $\left(v_{s}\right)_{m}$ is defined as shear wave velocity of soil model.

Meymand (1998) and Moss et al. (2010) explained that no governing equation can be written describing the entire soil-structure system, nor can dimensional analysis or similitude theory be directly applied to this complex system to achieve "true" model similarity. The viable scale modelling approach for application of scale model similitude, therefore, consists of identifying and successfully modelling the primary forces and processes in the system, while 
suppressing secondary effects, thus yielding an "adequate" model. Some researchers (e.g., Meymand, 1998; Turan, 2009; Moss et al., 2010) mentioned that adequate model for the dynamic soil-structure interaction simulation can be achieved as long as Cauchy condition is satisfied. Moreover, the strain scaling factor $\left(\lambda_{\varepsilon}\right)$ need be kept equal to one. Upon satisfaction of Cauchy condition, the result from substituting the value of $\left(v_{s}\right)_{p} /\left(v_{s}\right)_{m}$ is equal to one.

The objective of the scale modelling technique for shaking test program is to reach dynamic similarity, where model and prototype experience homologous forces. Geometrical analysis is the key for scale model representation in this test program. Two major principal test conditions provide and establish the scaling parameters. The first is that testing is conducted in a 1-g setting, which states model and prototype accelerations to be equal. Furthermore, a model soil with similar density to the prototype soil is required, since it keeps a constant component for the scaling relations. Additionally, shaking tests performed in cohesionless soil medium need be loose sand due to the effect of reduced stress in scaled model which can be compensated for by reduced density. Moreover, tests are conducted with uniform soil deposits of clay, whose undrained stressstrain response is independent of confining pressure, thus this may simplify the constitutive scaling requirements. By defining scaling conditions for density and acceleration, the mass, length, and time scale factors can all be expressed in terms of the geometric scaling factor $(\lambda)$, and a complete set of dimensionally correct scaling relations (ratio of prototype to model) can be derived for all variables being studied.

Scaled pile models can be expressed as having geometric, kinematic, and dynamic similarity to the prototype scale (Langhaar, 1951). It is important for the model pile to match with prototype model standards, therefore the aim of scale model is to reach an effective model pile design that can consider the principle factors of pile response including slenderness ratio (L/d), 
moment curvature relationship, flexural stiffness (EI), soil and pile stiffness, yielding behaviour, and natural frequency of vibration (Meymand, 1998). The applied geometric similarity result with the overall pile slenderness and relative surface area can be preserved in the model. Furthermore, the relative spacing and group interaction can be adhered with the model scale.

Iai (1989) derived a comprehensive set of scaling relations for a soil-pile-structure system under the dynamic loading by defining scaling conditions for density, acceleration, displacement, length, moment curvature of pile (EI) scale factors can all be expressed in terms of the geometric scaling factor $(\lambda)$, and a complete set of dimensionally correct scaling relations (ratio of prototype to model) can be derived for all variables being studied. The scaling relations for the variables contributing to the primary modes of system response are shown in Table 2.3 (e.g., Iai, 1989; Meymand, 1998; Turan and El Naggar, 2008; Moss et al., 2010; Sulaeman, 2010; Lee et al., 2012; Hokmabadi, 2014; Ling et al., 2014; Li et al., 2018).

Table 2.3: Scaling relations for shaking table in terms of geometric scaling factor $(\lambda)$ - (Iai, 1989)

\begin{tabular}{llllll}
\hline Mass Density & 1 & Acceleration & 1 & Length & $\lambda$ \\
Stiffness & $\lambda^{2}$ & Shear Wave Velocity & $\lambda^{1 / 2}$ & Stress & $\lambda$ \\
Modulus & $\lambda$ & Time & $\lambda^{1 / 2}$ & Strain & 1 \\
Displacement & $\lambda$ & Frequency & $\lambda^{-1 / 2}$ & EI & $\lambda^{5}$ \\
\hline
\end{tabular}

\subsection{Summary}

Local site conditions and soil-pile-structure interaction could have important effects on structural damage during earthquake. The amplification factor during the 1985 Mexico City Earthquake was estimated as large as about 8 on soft soil deposits in some parts of the city. This factor was about 3.5 on soft soils during the Loma Prieta Earthquake 1989. In the 1995 Kobe Earthquake, the ground motions were amplified by a factor of 1.5 to 2 times in areas with deep sedimentary layers. Analysis of Hanshin Expressway Bridge during the Kobe earthquake showed that SSI caused the 
effective period of the system to increase by almost $28 \%$, indicating that SSI may have been a contributing factor to the failure of the bridge. SSI was shown to have some important effects on the ground input motion and the seismic response of the structure, especially for short period structures. Accordingly, several building codes recommend a simplified linear method together with SDOF structure, which ignores important characteristics of SSI and local site effect including nonlinear response of soil.

For almost the last two decades, composite pile materials such as FRPs have been developed, however most of the studies were focused on the load transfer and flexural response of FRP piles under static loading. Results of these analyses revealed that composite piles may well be driven to reasonable capacities for load-bearing piles. It was also reported that driving forces may have minor influence on the flexural strength of CFFT piles. Concrete filled CFRP tubes with fibre orientation along the length of the pile were reported to demonstrate higher load carrying capacity whereas the unconfined concrete piles reached lower capacity (Murugan et al. 2017).

Field and laboratory tests presented in this chapter evaluated different aspects of soil-pilestructure interaction for pile foundations. These studies covered the effect of geometry of piles, foundation flexibility and rigidity, type of soil and relative density of soil, soil placement and layering, pile spacing (boundary effect) and installation technique, input motions, and impedance function for flexible and rigid foundations. The seismic performance and soil-pile interaction for hollow FRPs piles have not been investigated. Hence, this research developed a series of shaking table tests using laminar container system to study the seismic performance of hollow Carbon and Glass FRP group piles compared to traditional group piles in two altered mediums of liquefiable sand and soft clay. These shaking tests are employed rigorously to acquire parametric examination of FRP piles in laminar box system. 


\section{CHAPTER 3: EXPERIMENTAL PROGRAM}

\subsection{Introduction}

Deterioration of conventional pile materials overtime have raised concerns to develop materials with higher durability in order to minimise long term maintenance. Additionally, several devastating earthquakes causing significant economic loss and fatalities have been reported due to piles damage followed by superstructure failures. Consequently, the FRP pile behaviour under seismic loading remains a topic of research interest. Deeper insight into the response of pile foundations and pile-soil interaction under seismic loading promises to improve current design methodologies and avoid catastrophic failure during future earthquakes. Several studies have been dedicated to this problem, including shake table experiments, centrifuge tests and field tests. Considering the importance of earthquake in the design of foundation, newly introduced piling materials such as FRPs are still under investigation to fully understand their seismic response, and the concept of pile-soil interaction. To date, several studies on load transfer and flexural response of these piles under static loading have been investigated (e.g., Hosseini and Rayhani, 2017). However, seismic performance of these piles under dynamic loading is not well understood. This study aims at investigating the seismic response of hollow FRP piles compared to traditional piles using $1 \mathrm{~g}$ shaking table tests. Moreover, an advanced laminar soil container is designed to simulate the free field soil response by minimising the boundary effects. The use of such scale models is essential to simulate field conditions and comprehend the seismic response of model piles. This can offer the advantage of simulating complex systems under controlled conditions, and the opportunity to gain insight into the fundamental mechanisms operating in these systems. Hence, shaking table experimental program is undertaken to study the seismic response of the scaled 
models of hollow Carbon- and Glass Fibre-Reinforced Polymer (FRP) piles as well as traditional piles (e.g., aluminium) embedded in medium of liquefiable soil and soft clay.

This study consists of eight shaking table tests (4 phases), and each shaking test assesses the seismic performance of two different sets of 2 by 2 group piles (in terms of material difference) through six series of scaled earthquake input motions. Phase I is focused on seismic behaviour of end-bearing pile groups ( 2 by 2 ) made of CFRP, GFRP and aluminium piles in liquefiable sandy soil. Correspondingly, phase II examines response of frictional piles under identical procedure and site condition. Phase III and phase IV investigates behaviour of end-bearing and frictional group piles in uniform soft clay deposit.

This chapter describes experimental setup including preparation of shaking table test facility and controlling software, design and fabrication of model piles, instrumentation of pile and soil models and shaking container, calibration and monitoring devices, data acquisition system (DAQ), and interconnection of controlling DAQ software, followed by the design of the model soils and soil samples used in the test program. Ultimately, placement of piles and soil deposit along with applied input ground motions and a summary of shaking tests plan are discussed.

\subsection{Shaking Table Test Facility}

The shaking table system used in this study is a high-performance servo-hydraulic shaker that can apply 1-D horizontal base input displacements to models and model containers in response to an applied input voltage signal. The shaker slip-table is comprised of a rigid steel weldment supported and guided by two precision linear guides and four bearing cages incorporating recirculating roller bearings, and a payload mounting area of $2134 \mathrm{~mm}$ x $2032 \mathrm{~mm}$ which can accommodate a maximum payload mass of 10 tons and a maximum double amplitude (i.e., peak to peak) displacement of $300 \mathrm{~mm}$. The shaking is provided by a hydraulic actuator (MTS) with dual servo 
valves and shaking force of $98 \mathrm{kN}$ at the nominal operating frequency range 0-50 Hz. Figure 3.1 illustrates shaking table assembly including the base frame, slip table, linear bearing, hydraulic actuator, actuator base, and accumulators.

This shake table is designed and equipped with two laminar containers dimensions for simulating free-field boundary with two different internal dimensions of 1x1 meter and 2x2 meter at a fixed height of 1 meter. Figure 3.2 shows the assembly of the $1 \times 1$ meter laminar as chosen alternative to perform these seismic simulations. The container assembly is started by placing the 'Bottom Rail Screw Bushings' over the threaded holes in the shaker slip table, followed by placement of initial assembled ring with containing four bottom ring extrusions over the bushings. Upon installing the fixed ring, other laminar members were assembled by hand through proceeding one corner at a time. Each container is comprised of a stack of 27 laminates (or rings) supported and placed row by row on array of roller bearings at assigned orientation, spaced, and mounted within bearing cages (Figure 3.2). The laminates comprise the walls of the container and are manufactured from high strength aluminium alloy extrusions for high strength/stiffness and low mass. These thin rings were designed to form the boundary of the shear container, and to accommodate any continuous shear strain concentrations within soil deposits during shaking. The maximum relative displacement between adjacent rings is $7 \mathrm{~mm}$, which this can minimize volumetric strains due to membrane bulging or cling. These adjacent rings are separated by steel roller bearings for low friction and precise motion (Table 3.1). A 3.5 by $3.5 \mathrm{~m}$ flat latex membrane folded with a thickness of $0.5 \mathrm{~mm}$ was stretched freely inside the laminar container to act as waterproofing sheet to prevent soil or water penetration into the small spacing between laminae. The combination of the rubber membrane and minimal spacing of aluminium laminar can minimize the chance of tramping of membrane and deliver the desired lateral flexibility without 
bulging. This elastic material (i.e., latex) can also act as an interlayer material to minimize the inertia forces from surrounding thin aluminium laminar rings, which further induces the low sliding friction between laminaes. Figure 3.3 illustrates the fabricated latex membrane inside the laminar container as it is secured on top ring using clippers.

Table 3.1: Key properties of the SS10K-05- Shaking table

\begin{tabular}{|l|l|}
\hline Mass (Empty) & $529 \mathrm{~kg}(1163 \mathrm{lb})$ \\
\hline Type & $1 \mathrm{D}$ \\
\hline Internal Dimensions (L x W x H) & $1016 \mathrm{~mm} \times 1016 \mathrm{~mm} \times 1071 \mathrm{~mm}$ \\
\hline External Dimensions (L x W x H) & $1245 \mathrm{~mm} \times 1245 \mathrm{~mm}$ x $1071 \mathrm{~mm}$ \\
\hline Maximum Total Displacement (Top Ring) & $172 \mathrm{~mm}$ \\
\hline
\end{tabular}
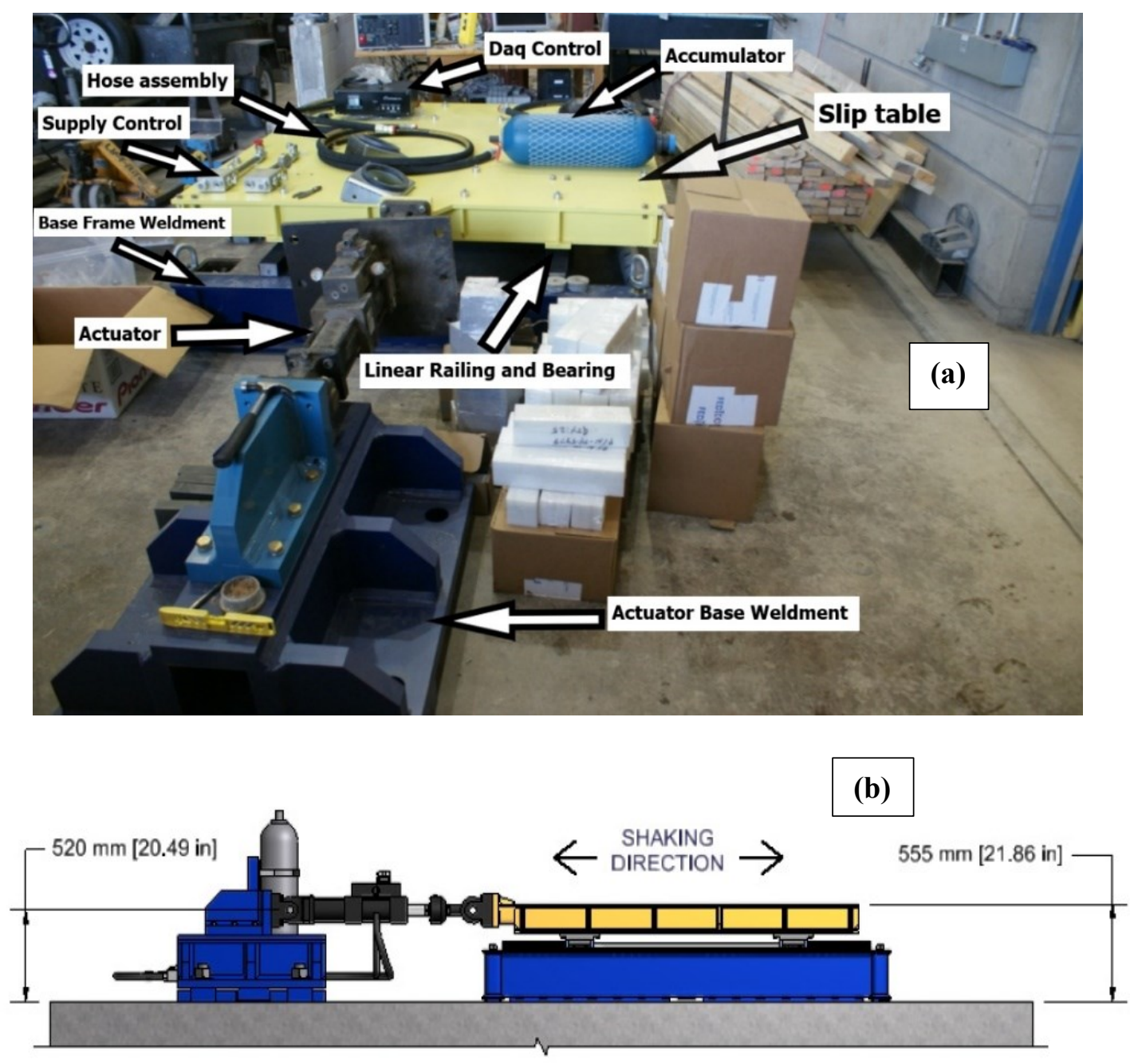

Figure 3.1 (a): Installed shake table by manufacturer; (b): Side view schematic drawing of shake table 

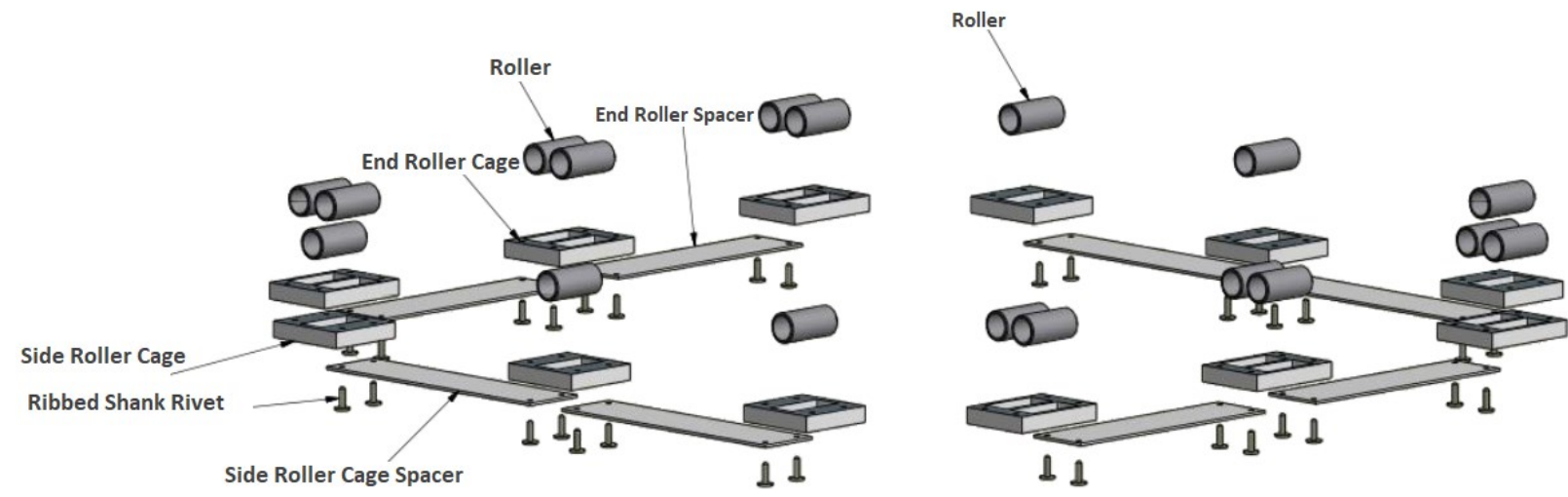

(a)
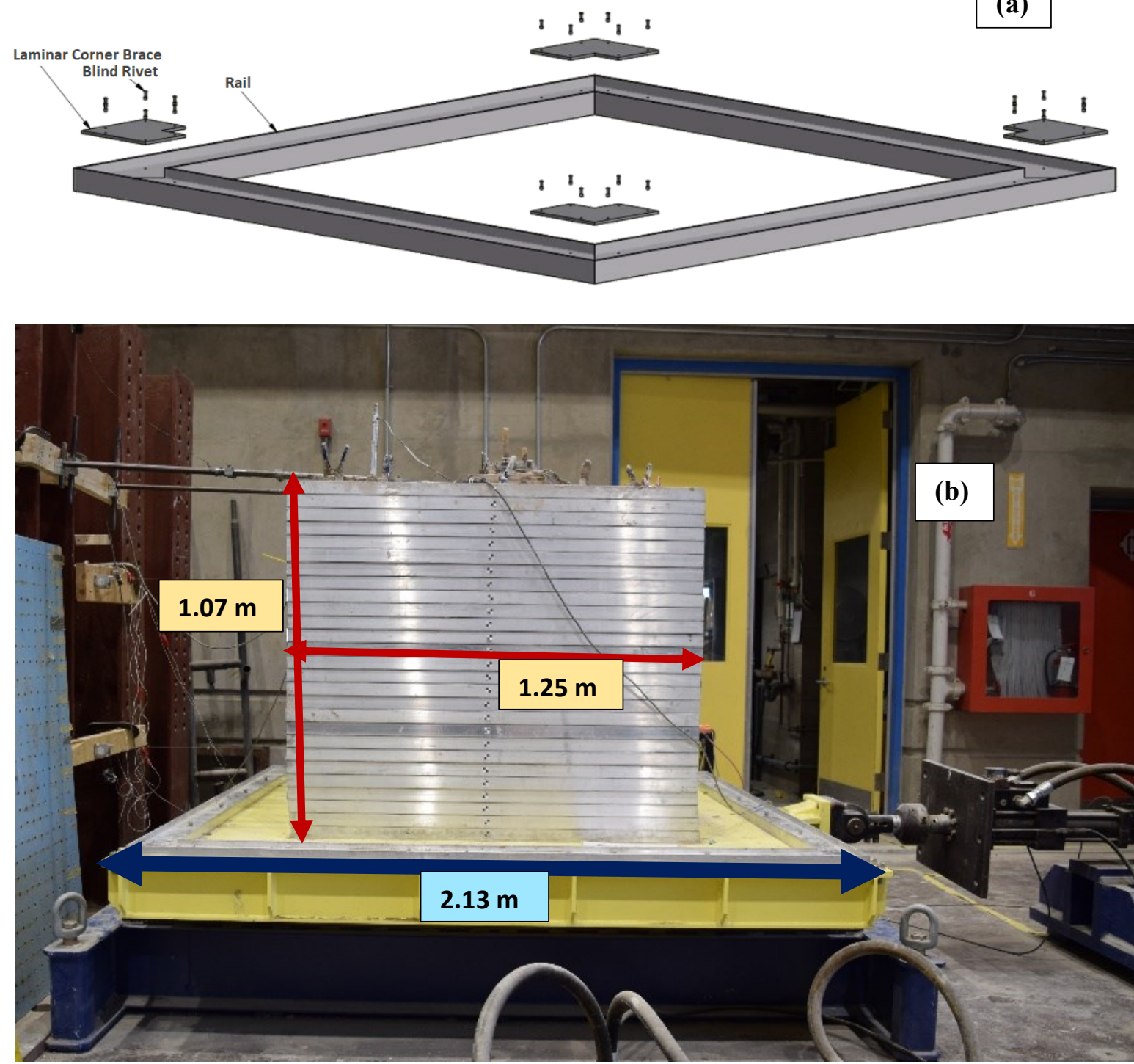

Figure 3.2: (a) Schematic drawings of laminar box container assembly (b) Final stage of assembled shake table with laminar container in Civil Engineering Lab, Carleton University 
Upon assembly of all shaking components, a servo-controller software (DaqControl) is employed to activate the shake table. This DaqControl contains two modules to monitor the shaker system which are recognized as a servo-amplifier (i.e., Moog G122-828-001), and an LVDT signal conditioner (i.e., Macro Sensors LVC-2500) (Figure 3.4). The shake table data acquisition system and its controlling software interconnect with the input motions which are converted to scaled voltages $(-1$ to +1$)$. After completing the controller software setup, all controlling mechanisms for the shake table were calibrated using a concrete block as a medium to adjust all software and hardware systems for the shaking test. Additionally, dummy shaking tests on a dry sand medium was conducted before initiating the main shaking tests to examine the functioning of the table and all sensors performance under scaled inputs motions. Activate

Accordingly, measurements from several dummy tests agreed with the predictions confirming that the dynamic shear stiffness similarity between the laminar container and the ideal free field behaviour of the soil deposit. As mentioned in chapter 2, the soil deposit has a fully nonlinear behaviour and its stiffness is changing under the cyclic loading; however, the stiffness of the container is constant during the shaking tests.

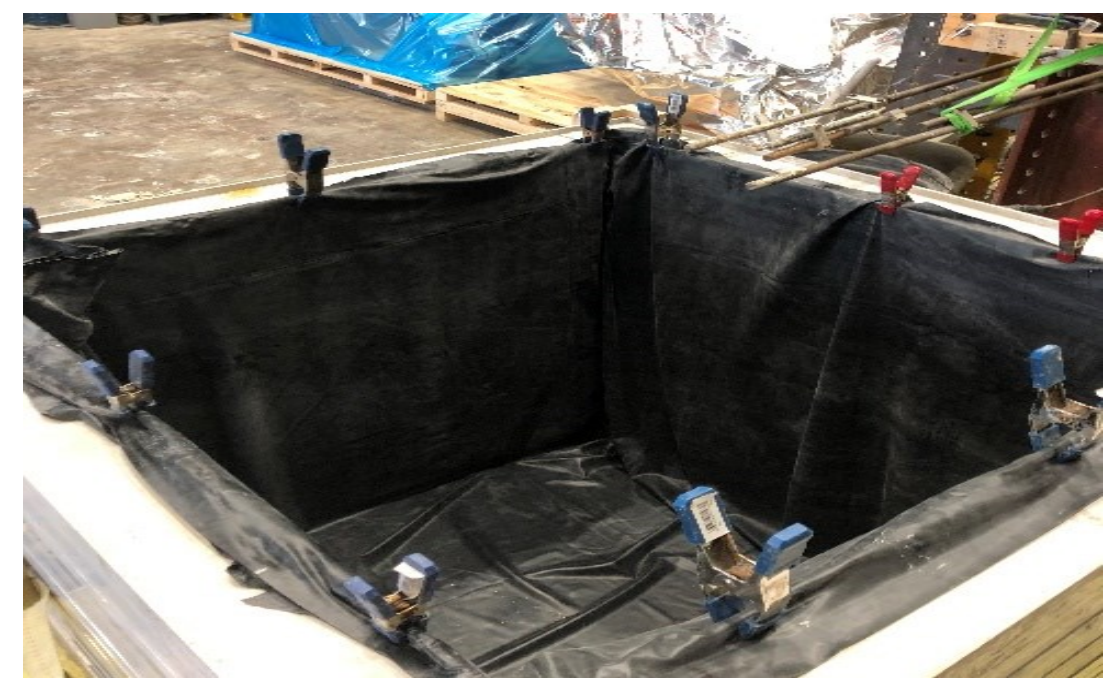

Figure 3.3: Latex membrane assembly for waterproofing the laminar container 


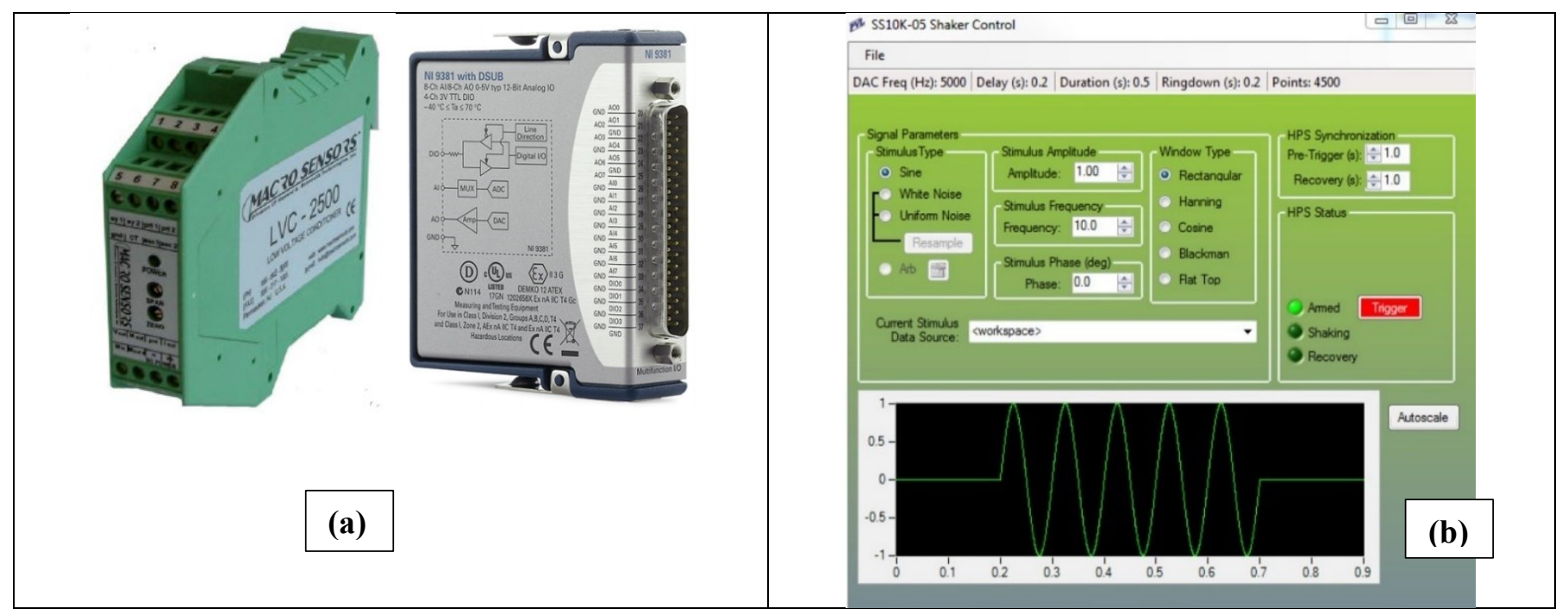

Figure 3.4: (a) Shake table data acquisition system (b) shaker control software (i.e., SS10K)

\subsection{Scale Factors for Shaking Table Testing}

The objective of the scale modeling procedure for this test program is to achieve what has been previously defined as "dynamic similarity", where both the model and prototype experience homologous forces. Adopting an appropriate scaling factor $(\lambda)$ for 1 -g shake test is one of the important steps in scale modelling for shaking table tests. While smaller scale factors could save cost, the precision of the results could be substantially reduced. Considering the specifications of the shaking table used here, a scaling factor of 1:10 provides the largest achievable scale model with $1 \times 1$ laminar container and all the facility limitations. Thus, a geometric scaling factor $(\lambda)$ of 1:10 was adopted for experimental shaking table tests in this study.

\subsection{Model Pile Foundations}

Several previous studies such as Tao et al. (1998), Maugeri et al. (2000), Chau et al. (2009), Ling et al. (2013), Tang et al. (2014) and Durante (2016) used common aluminium tubes, steel bars or pipes and reinforced concrete as model pile materials. Considering the high demand of using 
composite materials in piling industry, this study is primarily focused on investigating seismic response of hollow FRP (carbon or glass) piles in comparison to traditional piles (i.e., aluminium model piles- alloy 6061). Many materials were investigated to ascertain their suitability as a traditional model pile, and aluminium material is the only candidate that falls in the range of acceptable modulus, and can be configured as a thin wall section to meet the EI criterion. Several past researchers have also considered aluminium as model pile in shaking table tests (e.g., Yao, 1980; Finn and Gohl, 1992; Makris et al., 1996; Bathurst et al., 2007; Chen and Ueng, 2010; Bao et al., 2012; Azizkandi et al., 2020). Thus, by considering the geometric scaling factor $(\lambda)$ of 1:10, the model piles would have a diameter of approximately $55 \mathrm{~mm}$ with $\mathrm{L} / \mathrm{d}$ ratio of 18.2 and 13.6, for the end-bearing and frictional pile foundations, respectively.

The moment-curvature relation criterion represents pile response to lateral loading which is a function of the flexural rigidity and yielding behaviour. Since in the present study piles are intended to respond in the elastic range, this criterion is achieved by scaling the flexural rigidity (EI) of the piles according to Table $2.2\left(\lambda^{5}, \lambda=10\right)$ in addition to ensuring that the yielding point of the model pile is equal to or greater than the selected prototype. The rationale for such elastic assumption is that damage to above ground structures is much easier to detect and repair than damage to subsurface elements. Furthermore, by scaling the stiffness of the soil and pile consistently, the relative soil/pile stiffness parameter will be satisfied inevitably within the range of acceptable criteria with 5-10\% deviation from the target value for flexural rigidity due to model pile material variation. 


\subsubsection{Fabrication and Installation of Pile Groups}

As mentioned, a scale factor of 1:10 was used to determine the model pile sizes in order to derive a practical prototype of concrete-filled steel pipe piles in industry (Table 3.2). A series of hollow FRP piles were designed to be manufactured with commercially available carbon and glass FRP fabrics used for the reinforcement and retrofit of structural members. The raw material was roll of fibre fabrics with a uniaxial direction. The raw material becomes saturated in epoxy to form the FRP structure which can be shaped to several different geometric forms. The model carbon and glass FRP piles were manufactured using a steel tube in which the saturated fibre is fitted around the steel tube. The piles were then extracted from the steel tube after 48 hours of curing time, as per the manufacture guideline. In addition to FRP model piles, a traditional pile of prefabricated aluminium tube was selected based on the range of acceptable modulus to be configured as a thin wall section to meet the EI criterion and follow the scaling rule. With respect to axial performance, calculations of driving stresses, static loads, and stresses under dynamic loading were found not to exceed the buckling load. A view of the fabricated model piles are shown in Figures 3.5. The geometric properties of the model piles for all phases of shake table tests are summarized in Table 3.3 .

Table 3.2: Prototype pile geometric properties

\begin{tabular}{|l|l|}
\hline Outside Diameter (mm) & 550 \\
\hline Length (m) & $7-10$ \\
\hline Wall Thickness (mm) & 13 \\
\hline Modulus of Elasticity of steel (MPa) & 200,000 \\
\hline Modulus of Elasticity of concrete (GPa) & 28 \\
\hline
\end{tabular}


Table 3.3: Model pile geometric properties

\begin{tabular}{|c|c|c|c|c|c|}
\hline \multirow[b]{2}{*}{ Pile Material } & \multirow{2}{*}{$\begin{array}{c}\text { Outside } \\
\text { Diameter } \\
(\mathrm{mm})\end{array}$} & \multicolumn{2}{|c|}{ Length (mm) } & \multirow{2}{*}{$\begin{array}{c}\text { Wall } \\
\text { Thickness } \\
(\mathrm{mm})\end{array}$} & \multirow{2}{*}{$\begin{array}{l}\text { Modulus of } \\
\text { Elasticity } \\
\text { (MPa) }\end{array}$} \\
\hline & & End-bearing & Frictional & & \\
\hline CFRP & 55 & 1000 & 750 & 5.2 & 7000 \\
\hline GFRP & 55 & 1000 & 750 & 5.2 & 5800 \\
\hline Aluminium & 55 & 1000 & 750 & 5.2 & 7800 \\
\hline
\end{tabular}
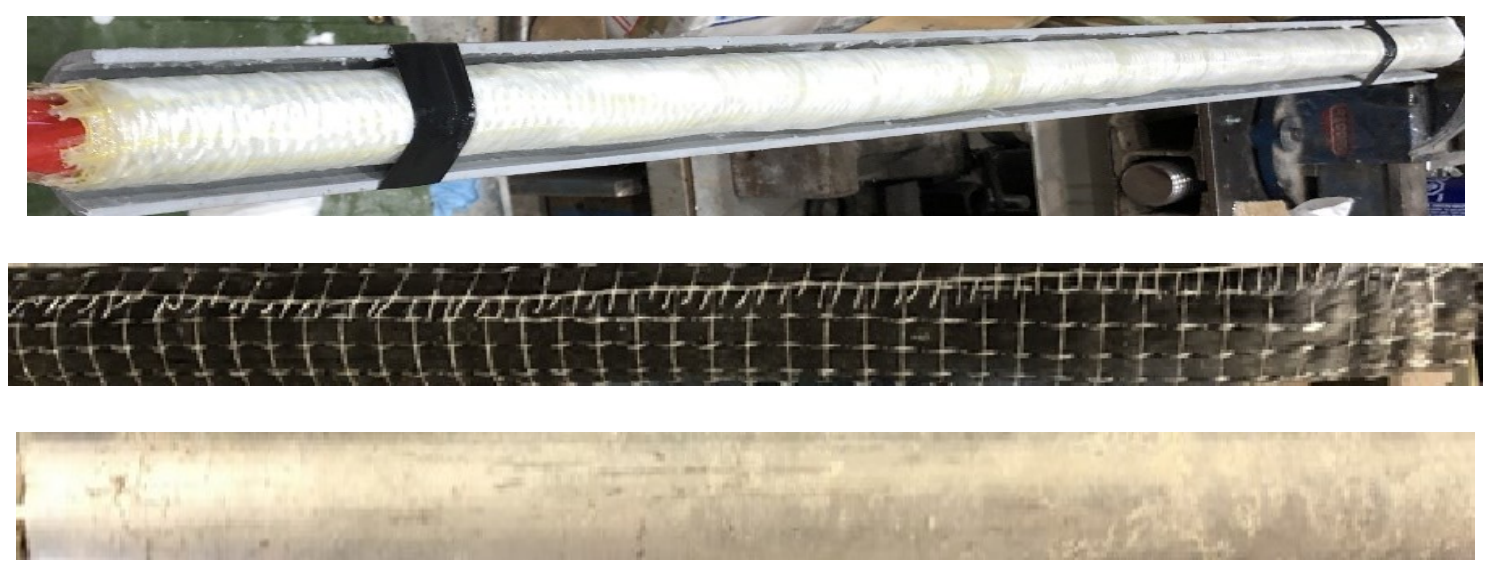

Figure 3.5: Glass FRP model pile (top), Carbon FRP pile (middle), and aluminium model pile (bottom)

After preparation of the individual FRP and aluminium piles, piles were divided into 2 by 2 group piles, following by instrumentation and positioning in the laminar container (Figure 3.6a). Each group consists of its own identical cap which is connected to a constant lead weight for all groups plus monitoring devices (e.g., accelerometer) to detect translation and rocking motions and any potential mobilization. Pile cap was fabricated by timber materials with dimensions of 260 $\mathrm{mm}$ by $260 \mathrm{~mm}$ and thickness of $76 \mathrm{~mm}$ (Figure 3.6). This cap section was fabricated with 4 holes, which piles are spaced at a centre-to-centre distance of about three times their diameter $(\mathrm{s} / \mathrm{d}=3)$ with respect to the boundary condition (i.e., known as common method used by Caltrans pile group design). 
Additionally, four-point bending tests were conducted using the Instron 5582 Universal Testing Machine (UTM) to measure the flexural properties of model piles (Figure 3.7). Results for the CFRP specimens demonstrated an ultimate flexural strength of $45 \mathrm{MPa}$, and flexural modulus of $7000 \mathrm{MPa}$. Similarly, flexural strength of GFRP specimens was measured at about $30 \mathrm{MPa}$ with a flexural modulus of about $5800 \mathrm{MPa}$. The ultimate flexural strength of identical aluminium specimens was measured approximately at $98 \mathrm{MPa}$ with a flexural modulus at about $7800 \mathrm{MPa}$.

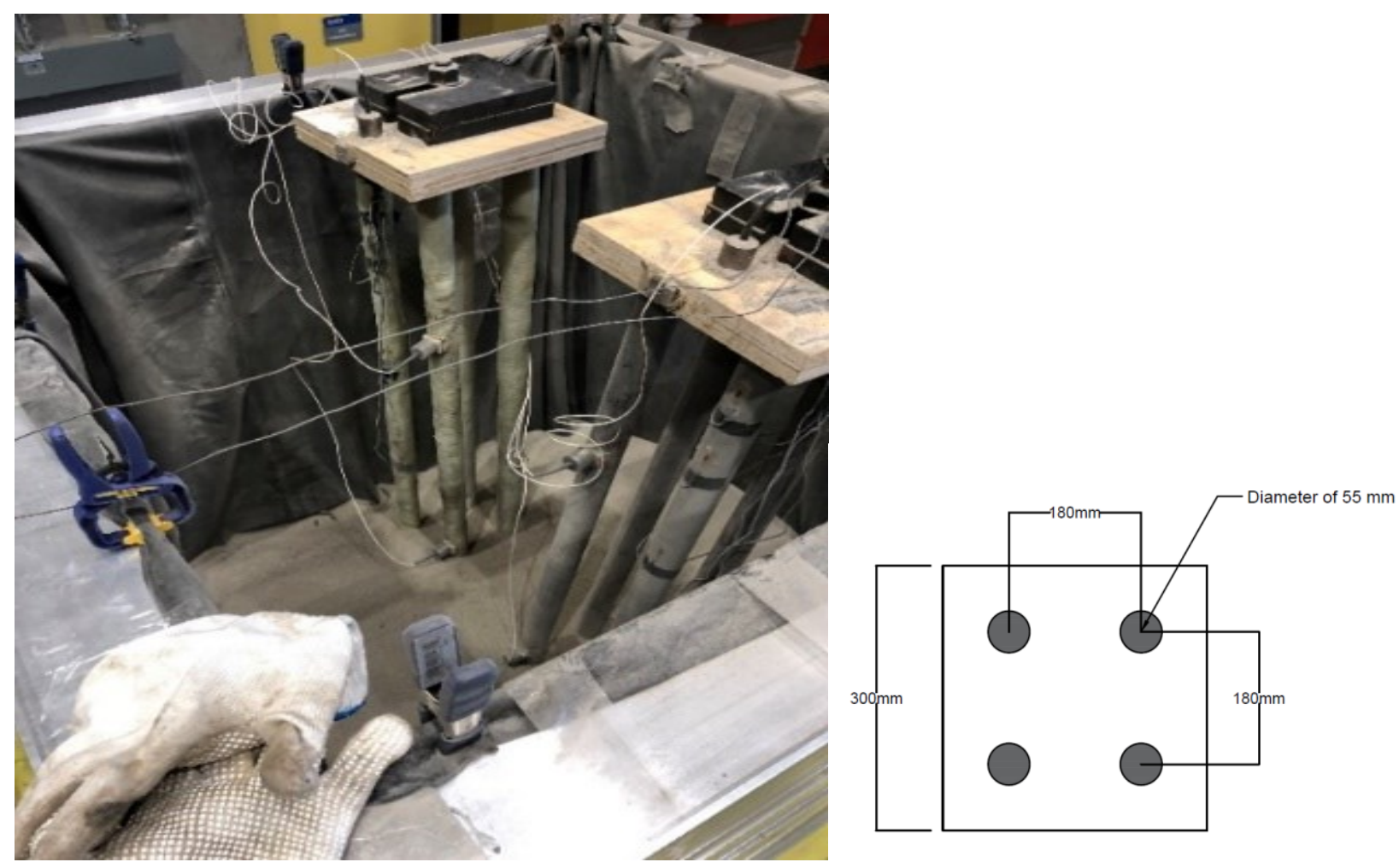

Figure 3.6: A view of GFRP group pile and aluminium group pile placed in shaking container, and a schematic drawing of pile cap dimensions 


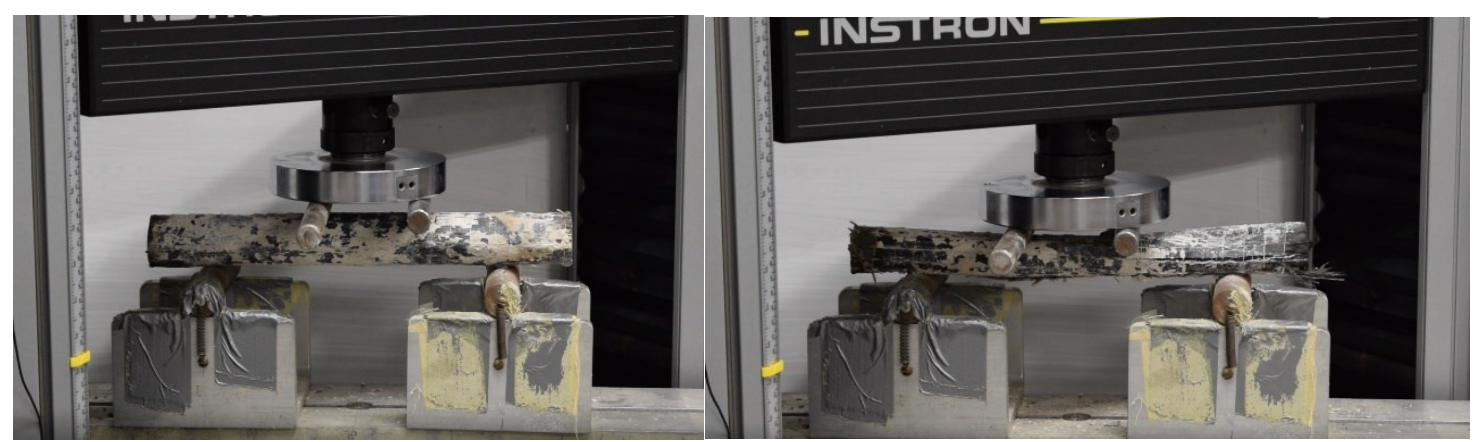

Figure 3.7: Four-point bending flexural test using UTS, before and after failure of CFRP member

\subsection{Experimental Instrumentation}

A series of strain gauges, accelerometers, linear variable differential transformers (LVDTs), and pore water pressure sensors were employed to monitor the response of the piles and the soil during shaking tests. In the next following sections, the role of each device along with calibration and installation procedure are explained.

\subsubsection{Accelerometers}

Accelerometers were used to measure the acceleration during seismic loading both in free-field and along the pile shaft and its cap/superstructure. These accelerometers were specially selected based on their sensitivity, small size, light weight (50 grams), waterproof and DC response (PCB Piezotronics; Model W393B04) and can be used within the frequency range of 0.06 to $450 \mathrm{~Hz}$ $( \pm 5 \%)$. (Figure 3.8). Additionally, these accelerometers consist of screw thread which are either glued to the specified positions of pile (i.e., using construction adhesive) and/or placed in free field which can move freely along the 1-D horizonal direction. 


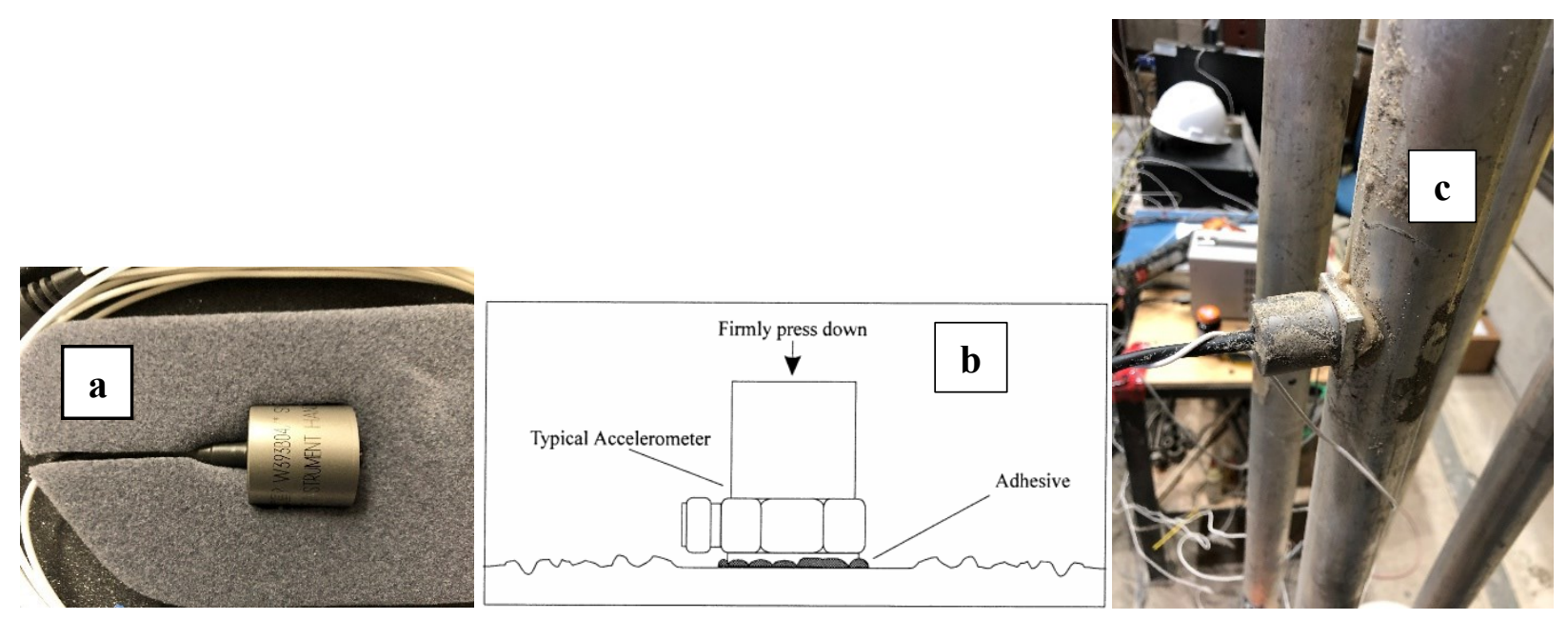

Figure 3.8: (a) PCB Accelerometer used in this research; (b) ICP signal conditioner system (c) sample of attached accelerometer to pile shaft

\subsubsection{LVDT}

Linear Variable Differential Transducers (LVDT) were used to estimate any horizonal displacements of the pile cap and model structure, and monitor possible vertical settlement at the soil surface. LVDTs were connected to a terminal block and, in turn, to a data acquisition (cDaq) module interconnected with the Chameleon software. Calibration of LVDTs were accomplished by recording the change in mechanical movement and the corresponding change in output voltage at a given supply voltage of 10 volts. The calibration factor was obtained by plotting the output voltage versus the corresponding movement.

\subsubsection{String Potentiometer}

Two string potentiometers (SP) were utilized to measure the change in displacements of laminar shear box. These string potentiometers consist of a primary and a secondary coil assembly fixed at a position. When they get displaced along the single axis; it sends a voltage output relative to the displacement (i.e., similar setup procedure as LVDTs). The string potentiometers were fixed on a stiff frame off the table while the string was extended and connected to the laminar frame at 
two locations with a distance of $200 \mathrm{~mm}$ and $800 \mathrm{~mm}$ from the bottom surface of the shake table as shown in Figure 3.9.

\subsubsection{Strain Gauges}

A pile in each group was instrumented with several strain gauges to detect bending strain profile of the pile. The model pile was equipped with 4 pairs of strain gauges fixed to opposing faces of the pile as shown in Figure 3.10. This gauge configuration is established to provide adequate resolution of the expected pile strain profiles specifically within the upper $1 / 3$ of the pile (Meymand, 1999). The exterior of the model pile was prepared by cleaning and smooth sanding the surface of gauge locations. Strain gauges (Model \# FLA-5-11-5L) were mounted to the exterior of the model piles using M-Bond 200 glue and Catalyst-c to heal the bond faster. After curing, the gauges and their attached wires were covered by multiple surface treatment of M-Coat polyurethane and a coat of silicon protection to waterproof the gauges. Upon mounting the strain gauges, the input wires were connected to spring terminal of National Instrument cDAQ module (NI-9235) which is a quarter-bridge strain gage module designed for dynamic strain measurement systems. Similarly, these strains gauges were pre-calibrated with a gauge factor of $2.11 \pm 1$ and characterized in the Chameleon software. 


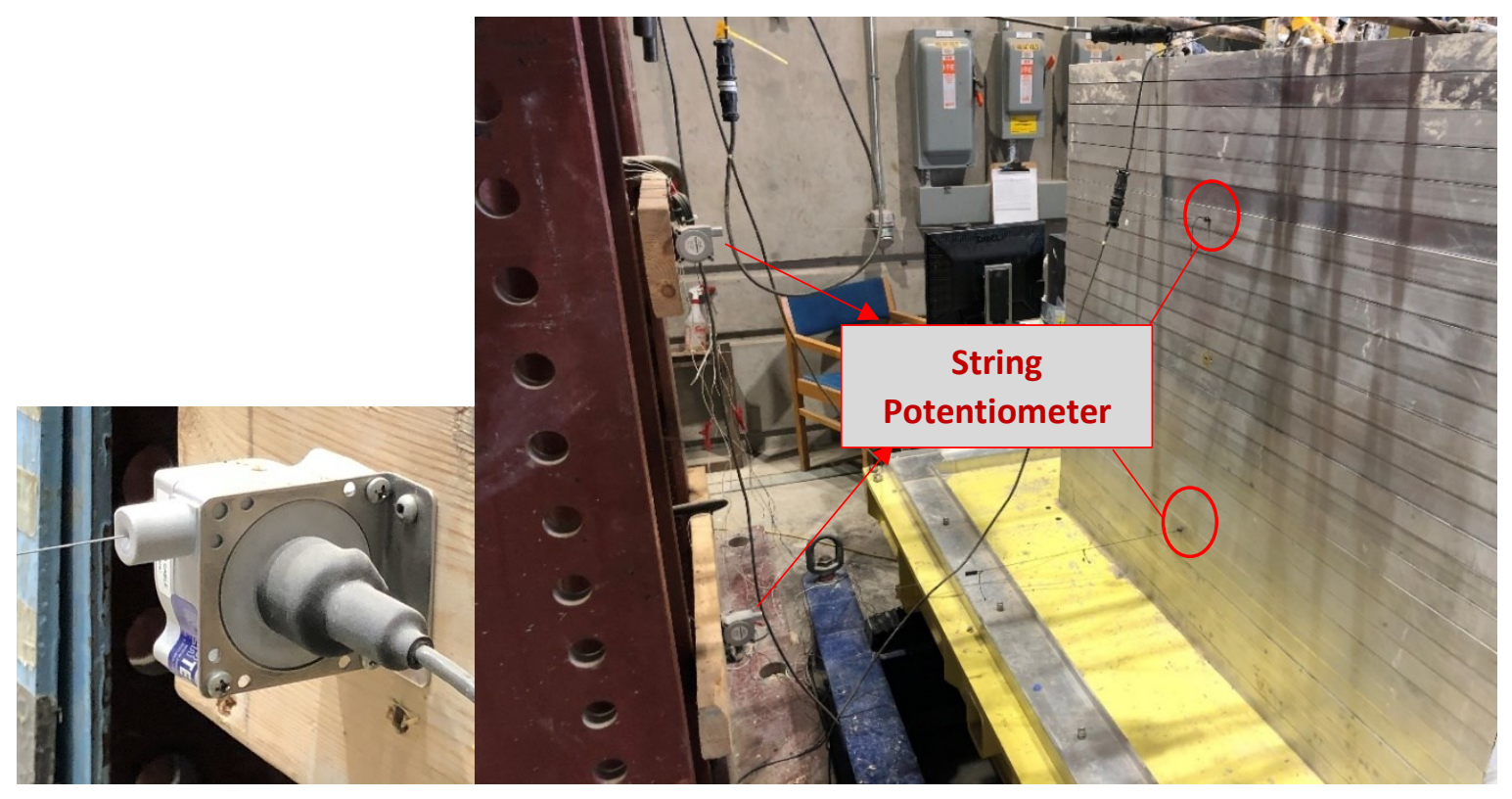

Figure 3.9: String Potentiometer used to measure displacement of laminar soil container
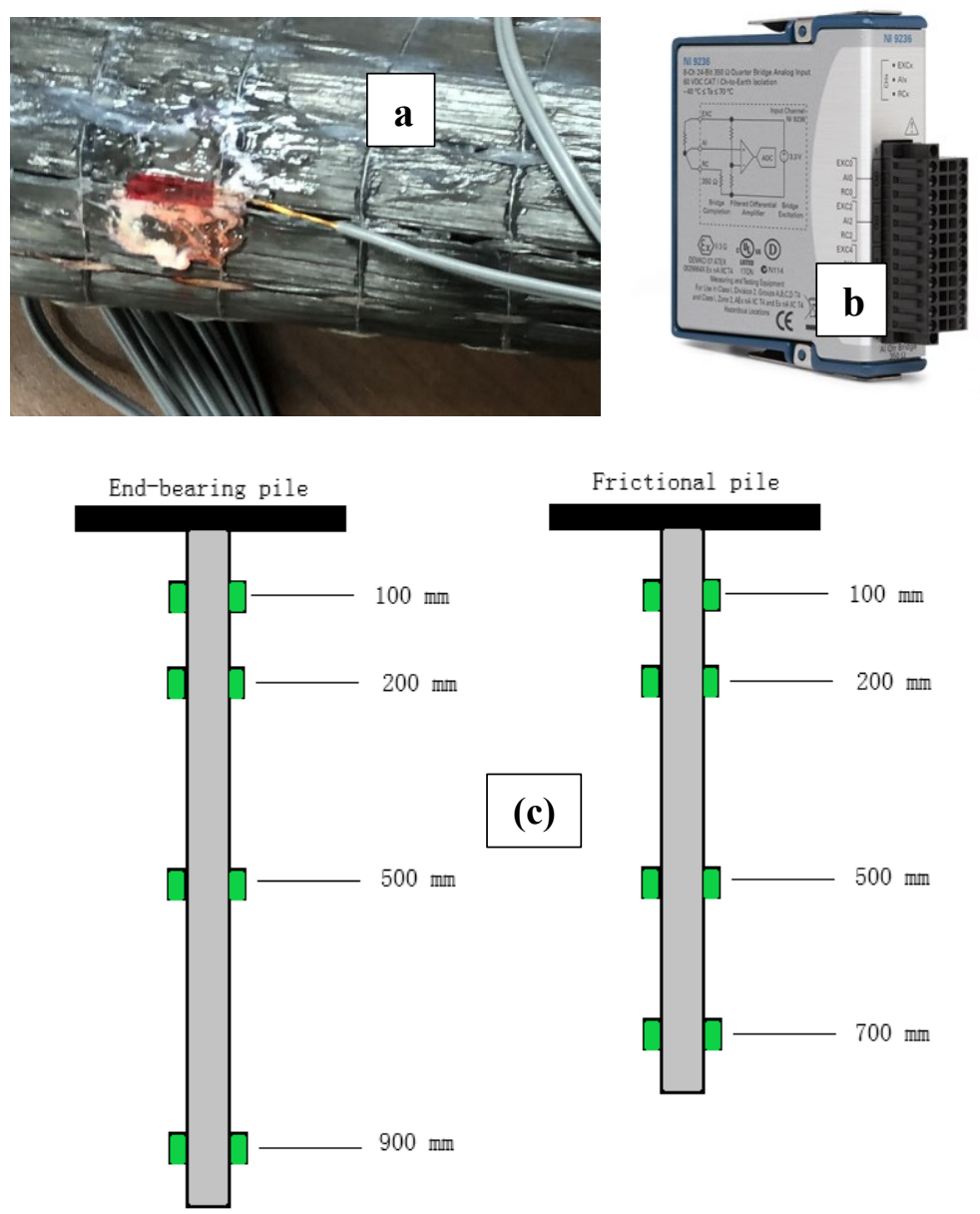

Figure 3.10: (a) Glued bending strain gauge on pile shaft; (b) cDAQ module for strain gauge sensors;(c) location of attached strain gauges along the pile shaft 


\subsubsection{Piezometers}

A standard vibrating wire piezometer (i.e., Pore Water Pressure, PWP sensor) manufactured by RST (model \# VW2106) was used to record and monitor the change in pore water pressure of the soil during each test. Each sensor was connected to a portable vibrating wire reader/logger to communicate and interpret an engineering value (Figure 3.11). These pore water pressure sensors were positioned vertically and within soil model at the same distance close to surface and base of model piles and secured wires outside the laminar container to estimate pore water pressure distribution with depth.

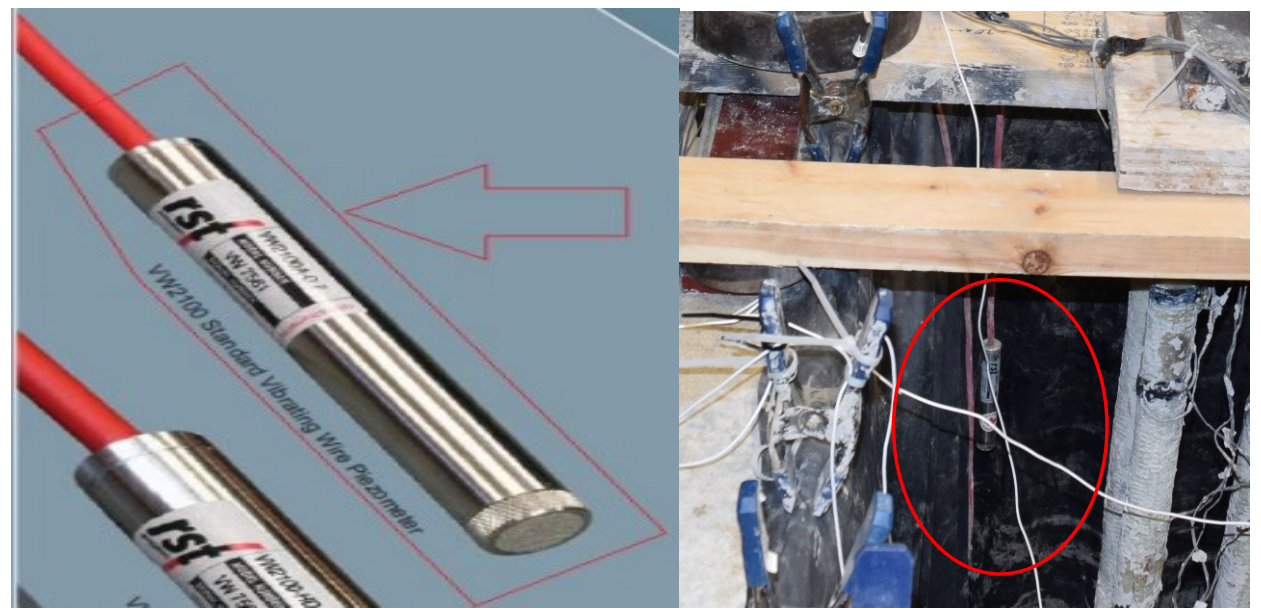

Figure 3.11: Standard vibrating wire piezometer

\subsubsection{Summary of Instrumentation Settings}

As discussed, several instruments and sensors including accelerometers, LVDTs and strain gauges were utilized to monitor these seismic tests. A total of eight accelerometer devices were positioned accordingly within the soil and across the shaft of model piles and its pile cap to monitor the shaking tests. These accelerometers are divided as follows: two accelerometers were positioned within the soil at a depth of $200 \mathrm{~mm}$ (BS) and about 700-850 mm (CB) (i.e., depending on the test condition for frictional or end-bearing piles) to record the free field motions in the soil. 
Correspondingly, two accelerometers were installed on a pile shaft in each group at the same depths as the free field accelerometers to measure the pile's response and pile-soil interaction effects. Also, one accelerometer was mounted on the pile cap of each group pile and on head masses to detect possible translation and rocking motions. String potentiometers were also fastened to the laminar box at similar depths (BS and $\mathrm{CB}$ ) to record the displacement along the laminar box, and additionally two LVDTs were positioned using a threaded plate attached to surface of the pile cap in horizontal direction to record any potential flexibility induced by the piles during shaking. Furthermore, an LVDT was fixed vertically on a metal plate placed on surface of the soil to record any soil settlement during each shaking excitation. Two piezometers were located inside the laminar container at similar depths as the $\mathrm{BS}$ and $\mathrm{CB}$ sensors to measure the change in pore water pressure during each excitation in sand. Lastly, a total of eight bending strain gauges were divided in groups of four mounted on both sides of individual model pile in each pile group to measure the strain and consequently develop a bending moment profile and investigate the influence of ground motion and pile-soil interaction on piles' response during different shaking events. Figure 3.12 illustrates the instruments positions inside the laminar container.

\subsection{Model Pile Placement}

Before placing the instrumented group piles and soil inside the container, it is essential to brace laminar rings by a wooden frame to align the upper extrusions with the bottom ring extrusion. This procedure is followed by complete reassessment, power test, software check of all sensors including accelerometers, strain gauges, LVDTs, and PWP sensors before sample preparation and testing. In both cases of end-bearing and frictional group piles, the model piles were placed at 
measured spacing followed by a temporary timber frame to avoid any movement during setting of soil deposits (i.e., synthetic clay or sand).
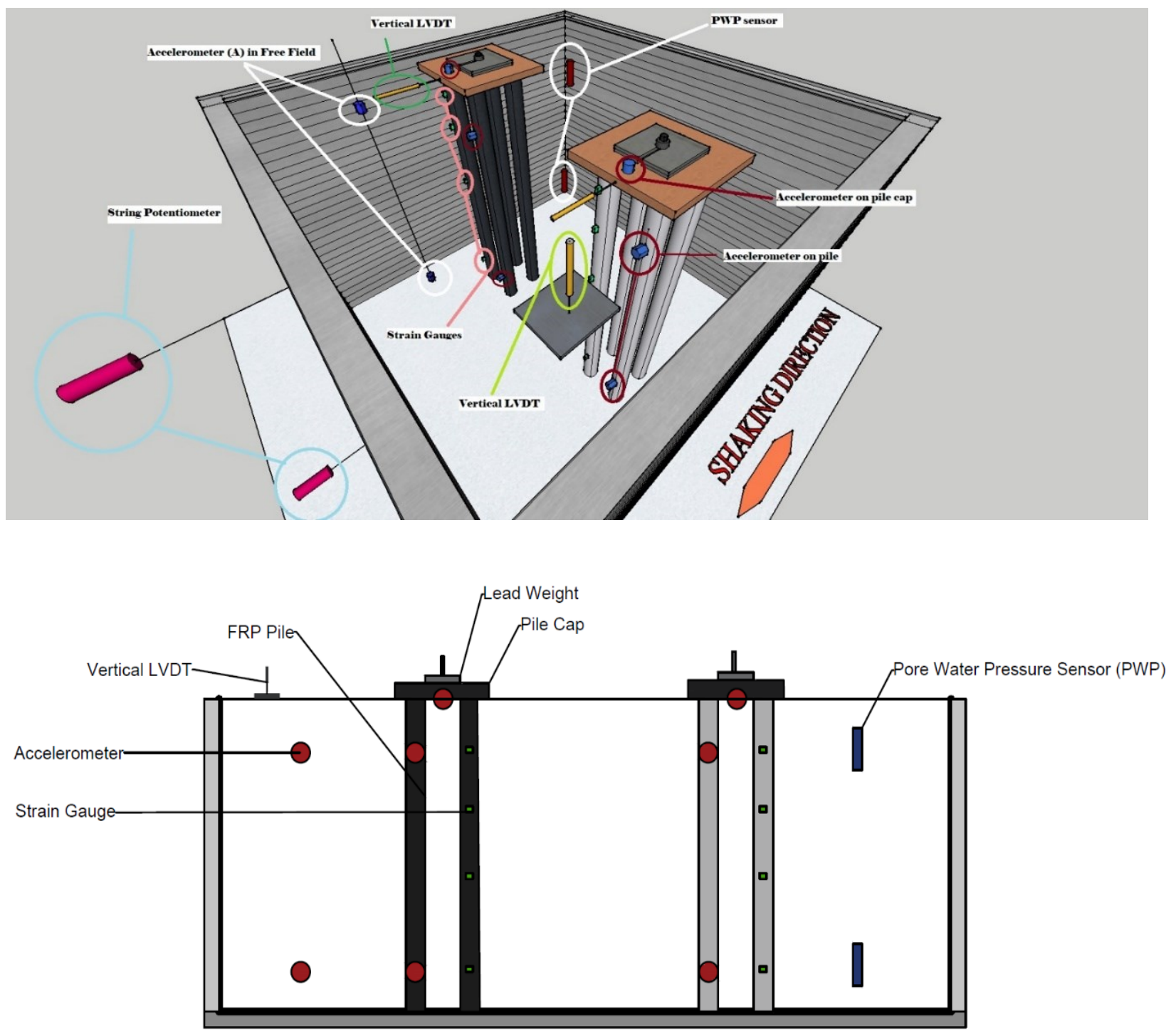

Figure 3.12: Front and side view of instruments positions inside the laminar container

The end-bearing group piles were embedded within the soil medium and placed on thin timber plates at the bottom of container to provide a closed end-bearing condition. This was also used to prevent any possible sliding between the piles and the container base plate (simulating the bedrock) during the shaking table test. The frictional piles were placed within the soil at the depth 
of about $750 \mathrm{~mm}$. The final setup of the tests included final mounting of the displacement transducers (i.e., LVDTs, positioned vertically and horizontally) and accelerometers at the pile cap. Additionally, two pore water pressure sensors were placed in free field in the sandy soil to monitor the liquefaction progress. Correspondingly, pile caps were rested on a temporary frame on top ring of laminars (Figure 3.13c). After temporary bracing of the pile caps, the soil layers, either sand or clay, were deposited in the laminar container. The following section will further explain the model soil properties, prepration and placement method in laminar container.
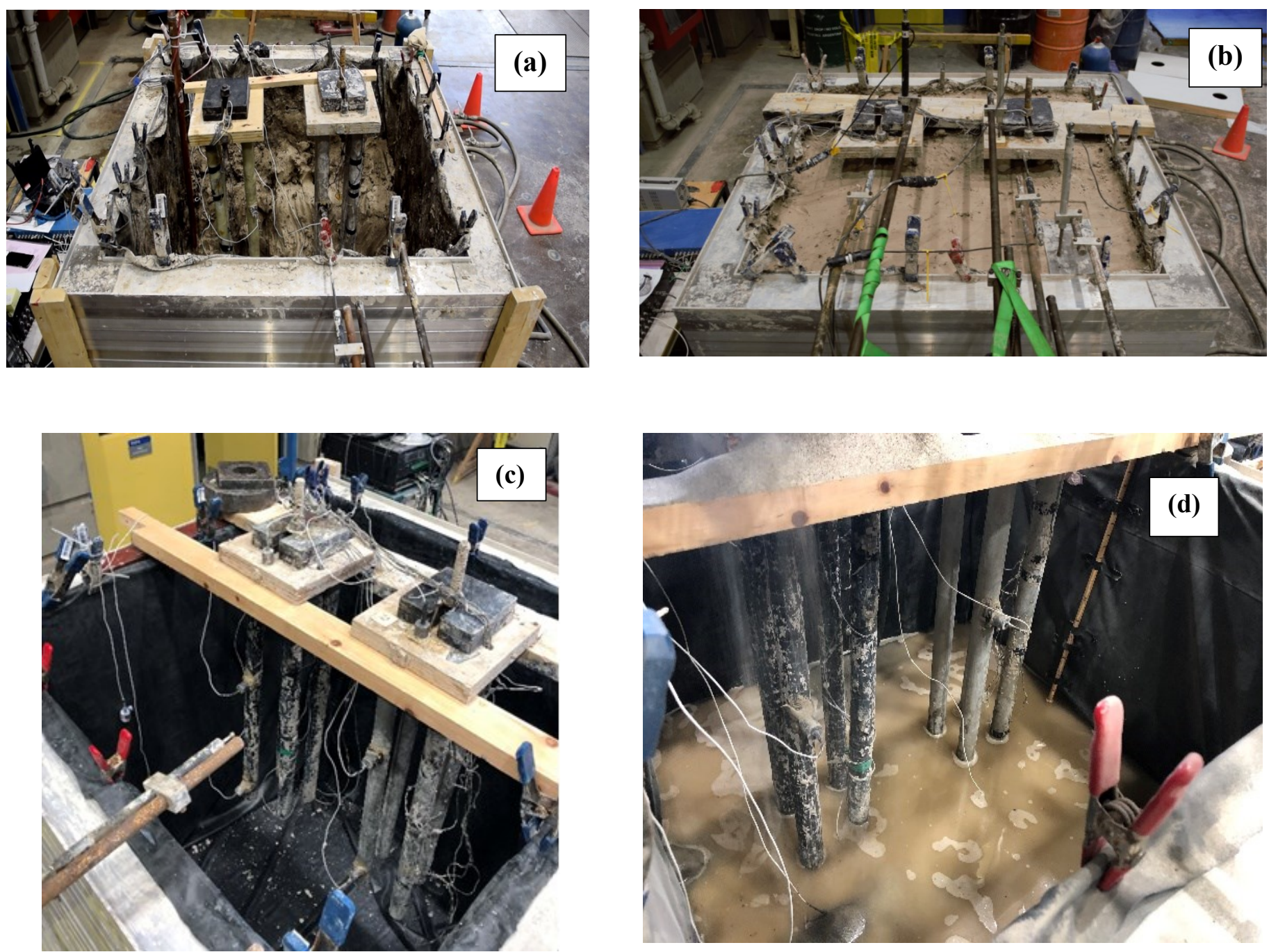

Figure 3.13: (a) \&(b) Placement of model piles in synthetic clay; (c) \&(d) placement of model piles in sand 


\subsection{Model Soils}

\subsubsection{Clay Soil}

The use of a reconstituted soil or field soil as the model soil is a common method used in centrifuge testing, where a natural soil is mined and then mixed with water to form a slurry that can easily be placed in the test container. Consequently, the soil is then consolidated to reach the aimed strength profile. The consolidation process offers the advantage of fixing anisotropy and a stress history into the soil. Nevertheless, this technique was not feasible and attainable in the current shaking table tests due to large scale of the shaking container and also, the extensive time that is required for consolidation in a 1-g environment. However, the Geoengineering Group at Carleton University have tried to extract large undisturbed sample of local soft clay (i.e., Leda clay), however upon trial, it was not possible to remove the sample intact from the steel cast, and place the undisturbed soil sample inside the laminar container (Fig. 3.14).

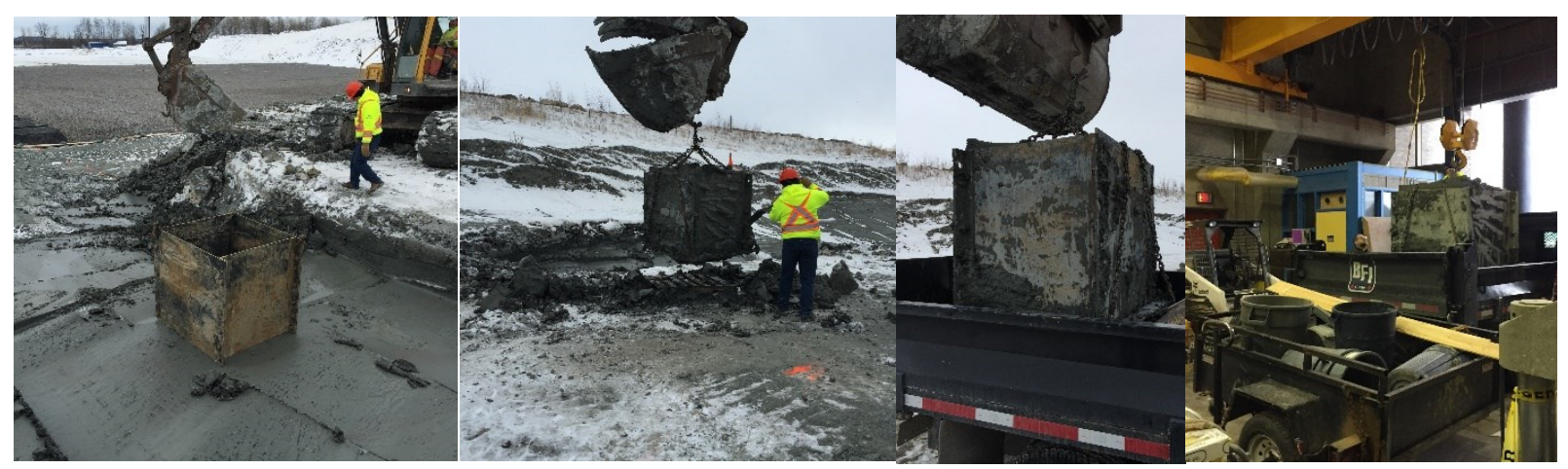

Figure 3.14: Extracted undisturbed soft clay for shaking table tests at Carleton University

On the other hand, the reconstituted soil cannot satisfy the scale modelling requirement specifically the undrained shear strength and dynamic shear modulus (Kana et al., 1986; Meymand, 1998). Therefore, it is essential to develop a synthetic model soil as the soil medium. Accordingly, several researchers developed numerous types of synthetic clay (e.g., Blaney and 
Mallow, 1987; and Iskander et al. 2002) and established model soil for testing in 1-g environment. A synthetic soil can follow the in-situ soil properties and behaviour including heterogeneity, anisotropy, fabric, and stress history. Tavenas et al. (1973) developed a synthetic soil mix using kaolinite, Portland Cement, and bentonite to simulate a brittle Lake Champlain clay. Several other researchers (Kubo, 1969; Liu and Chen, 1991; Dou and Byrne, 1996; Tao et al., 1998; Moss et al., 2010) conducted model pile testing using combination of various components including kaolinite, bentonite, supersil, plastellina, aerosil, veegum, silicon gum, plasticine, silts, and reconstituted clays. Seed and Clough (1963) developed a synthetic clay using kaolinite and bentonite to simulate the seismic response of embankments using shaking table tests. The mixture components ratio was 3 kaolinite and 1 bentonite, with almost $200 \%$ water content which revealed thixotropy. Hokmabadi (2014) compared three different mixture ratios of kaolinite, bentonite, fly ash Class F, and water. The finalized mixture was $60 \%$ kaolinite, $20 \%$ bentonite, $20 \%$ fly ash, and $120 \%$ water. The resulting mixture was convenient for placement and revealed anticipated shear modulus.

The principle soil criteria for model tests are undrained shear strength and shear modulus (or shear wave velocity) with respect to the protype soil properties. If the elastic response of both the free-field soil and the soil-pile system is desired, the soil shear modulus should be properly modeled. If the inelastic response of the soil-pile system is desired, then the undrained shear strength should be emphasized. If the full non-linear system response is desired, then both criteria must be satisfied simultaneously (Iai,1989; Meymand, 1998; Yasuda et al., 2000; Rayhani, 2007; Moss et al., 2010; Haeri et al., 2012; Hokmabadi et al., 2014). 


\subsubsection{Clay Sample}

A synthetic clay mixture was considered to provide soft clay soil medium characteristics for the shaking table tests. Similar synthetic mixtures recipes were used by several researchers such as Kana et al. (1986), Meymand (1998), Rayhani (2007), Moss et al. (2010), Hokmabadi (2014), Goktepe (2019), and they have stated that reconstituted soil from field could not satisfy the scale modelling criterion of undrained shear strength and shear wave velocity in shaking table tests whereas synthetic clay mixture can provide scale modelling criterion for the desired shear wave velocity and shear strength. Several synthetic mixtures were examined to follow the scale design. These mixtures were also compared to the prototype soil of soft clay.

The development of a model clay soil for this research project commenced in past research studies with initial mix designs. The proportion of mix components used in the shaking table tests include kaolinite and bentonite powders with specified amount of $70 \%$ and $30 \%$ respectively, along with an additional $100 \%$ water. This water content was expected to provide the key elements of mixability and pumpability for shake table model testing. Upon hand mixing the soil in small batches of 19 Litre buckets using hand drill mixer and also ensuring a homogenous mix sample, the synthetic clays were covered in 55 gal barrels and allowed to cure inside the sealed container. It was observed that this curing process allowed a more even distribution of water throughout the soil (in terms of consistency) (Figure 3.15).

The model soil had a unit weight of $1.43 \mathrm{Mg} / \mathrm{m}^{3}$. The undrained shear strength of the mix at a cure age of five days was determined to be $6 \mathrm{kPa}$, and the shear wave velocity at the same cure age was estimated at about $35 \mathrm{~m} / \mathrm{s}$. This benchmark cure age was used as it was expected that the time from soil placement to time of testing and the time between tests would be approximately five days. The prototype values implied by these model properties with a geometric scaling factor 
of 10 are a static undrained shear strength of $55 \mathrm{kPa}$ and a shear wave velocity of $110 \mathrm{~m} / \mathrm{s}$. These values agree with prototype parameters adopted for this research, and the model soil was therefore found to constitute an adequate scale model of a high plasticity soft clay.

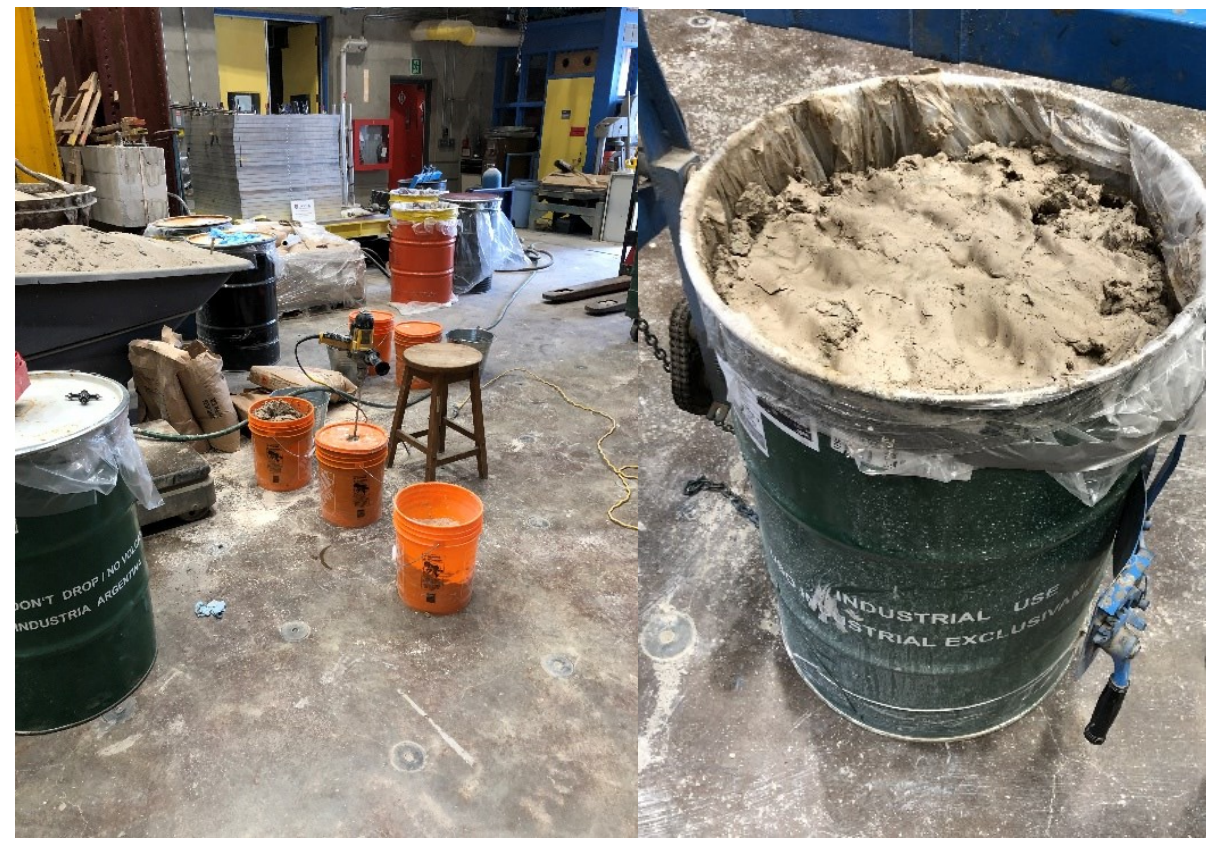

Figure 3.15: Synthetic clay mixed in buckets and prepared for model soil

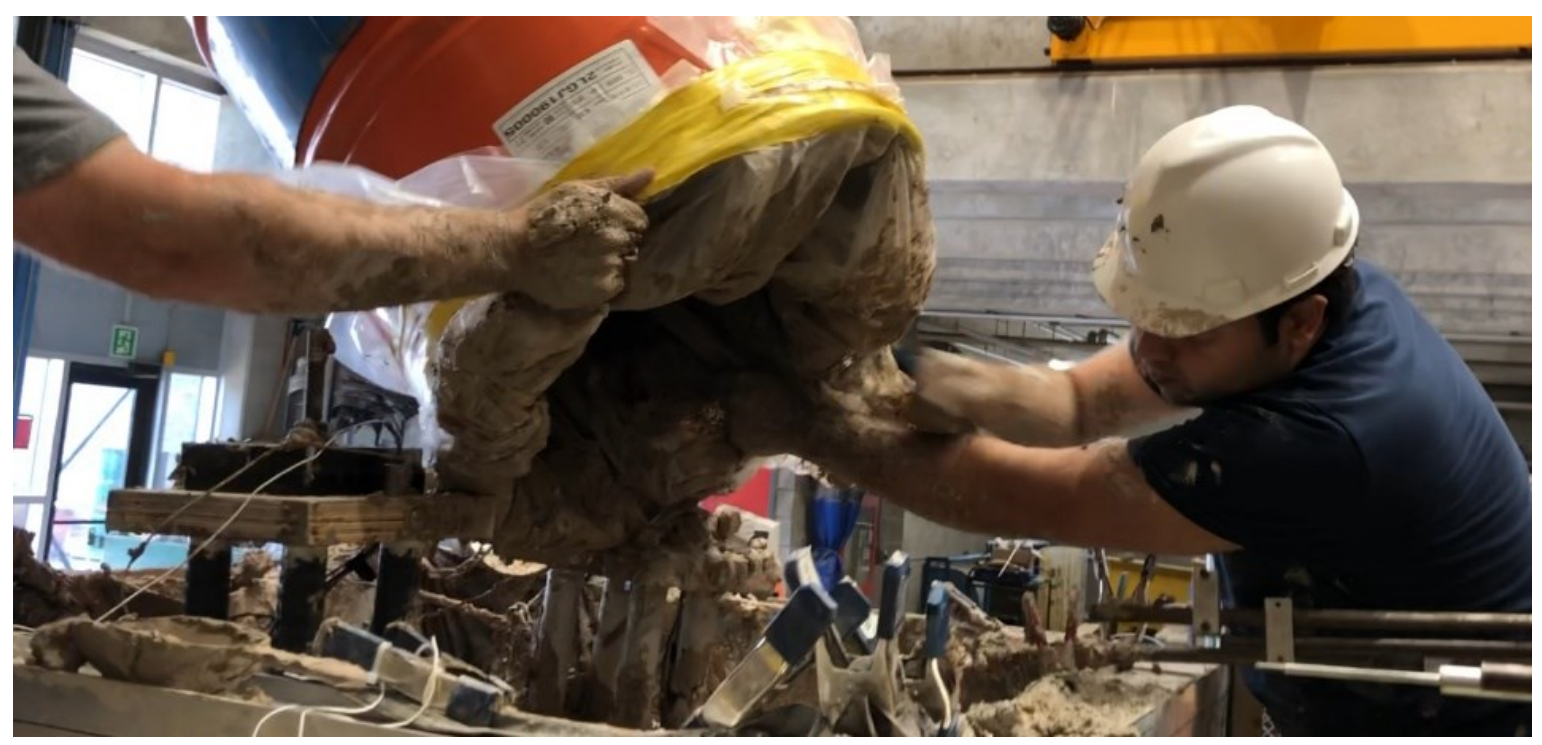

Figure 3.16: Preparation process for transferring synthetic clay inside laminar container 
Upon placing the model piles and mounting instruments, the mixture was then placed into the laminar shear container in several layers using drum lifter to provide easier handing in placing the synthetic clay. Ultimately, an effort was made to minimise entrapped air and to provide a uniform soil medium during placement (Figure 3.16). After placement and preparation of shaking tests components (Figure 3.17), the system was set for employing earthquake excitation.

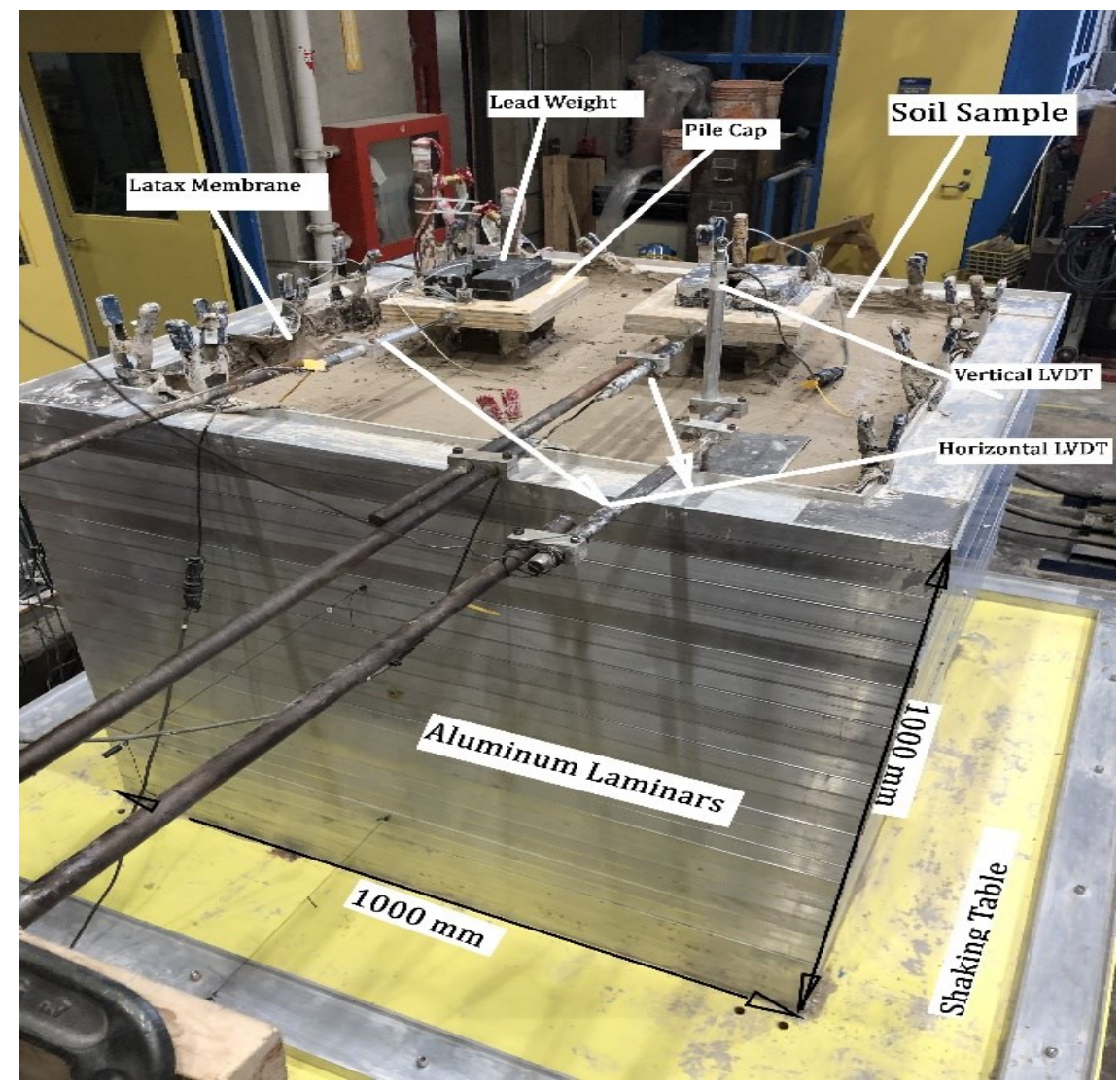

Figure 3.17: Various components of the shaking table models in synthetic clay medium with the group pile foundation adopted in this study

\subsubsection{Sand Soil}

Post-earthquake investigations indicated that pile foundations suffer severe damage due to liquefaction-induced lateral spreading. Such damages are observed in many strong earthquakes in 
the past such as the 1964 Niigata Earthquake, the 1974 Alaska Earthquake, the 1989 Loma Prieta Earthquake and the 1995 Kobe Earthquake. As a result, the seismic response of piles in cohesionless soils remain a topic of research interest as more composite piles such as FRP piles are being introduced in foundation industry. Mogami and Kubo (1953) used liquefaction term to tag and point out soil deformation due to monotonic, transient, or repeated disturbance of saturated cohesionless soil under undrained conditions, which is retrieved by development of excess pore water pressure. Cohesionless soils tend to densify under static and/or cyclic loading in dry condition. However, occurrence of rapid loading under undrained condition in saturated cohesionless soil may cause generation of excess pore water pressure and consequently reduction to effective stress. Scaled laboratory tests are used to comprehend the response of various foundations during liquefaction in controlled environment. Poorly graded sand was often used to simulate the liquefiable sand in model tests (e.g., Lie and Chen, 1991; Nasr, 2014; Mashhouda et al. 2018; Li et al. 2018).

\subsubsection{Sand Sample}

The model sand soil was a poorly graded sand (SP) with a fine content of less than $5 \%$ that was used to explore the seismic response of FRP piles and conventional piles in cohesionless soils. Figure 3.18 shows the particle size distribution of the cohesionless soil used in this study. The sand sample was a medium angular dry sand with uniformity coefficient of $\left(\mathrm{C}_{\mathrm{u}}\right)$ of 2.30 and coefficient of curvature $\left(\mathrm{C}_{\mathrm{c}}\right)$ of 1.26 . Additionally, the specific gravity $\left(\mathrm{G}_{\mathrm{s}}\right)$ of the sand was measured at 2.66 as per ASTM D854. The maximum and minimum void ratios determined according to the ASTM test standards (D4253, and D4254) are 0.845 and 0.504, respectively. Furthermore, the maximum dry density of the soil was measured at $1.67 \mathrm{Mg} / \mathrm{m}^{3}$ with the optimum water content of $10.8 \%$ as per ASTM D698 for standard Proctor test. 


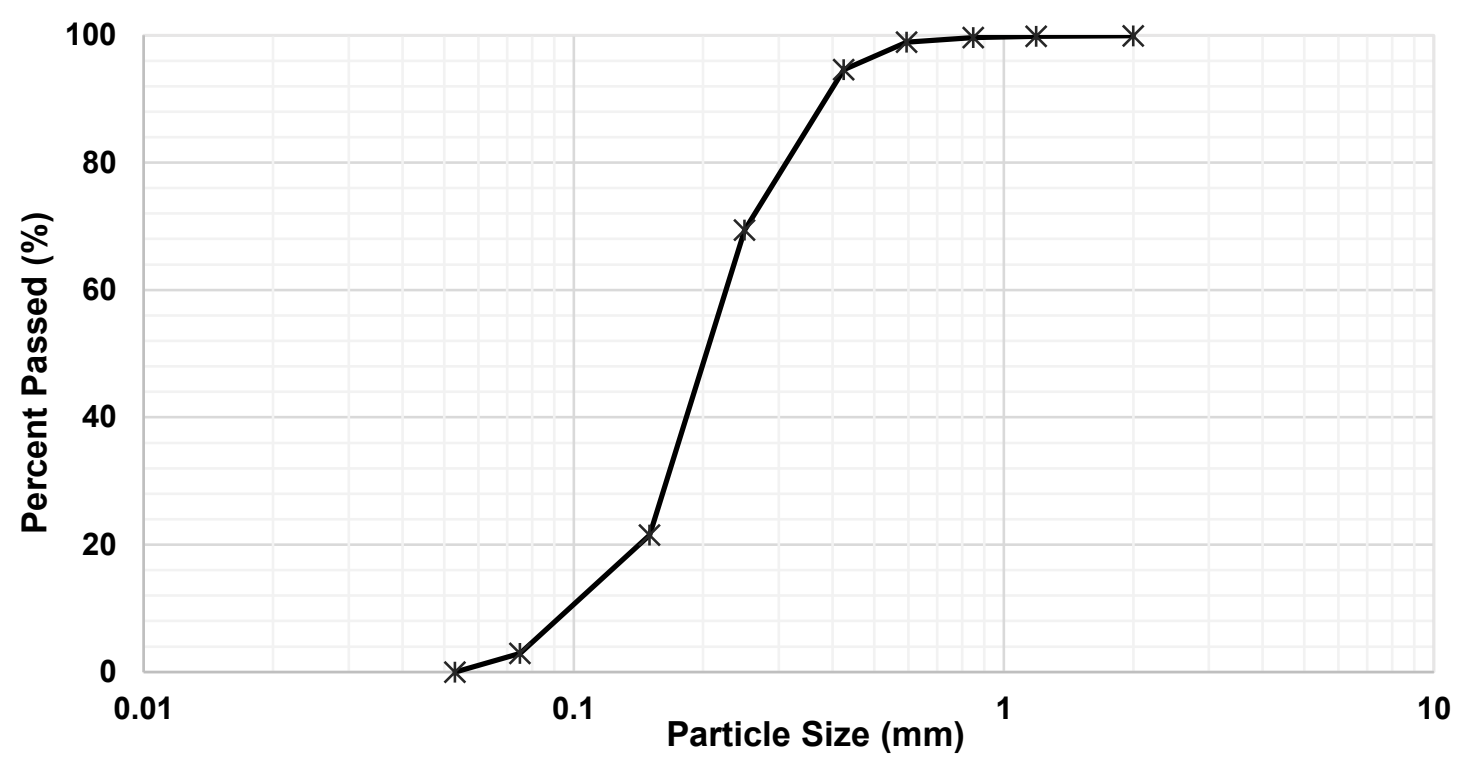

Figure 3.18: Particle size distribution of the test soil

Sand samples for shaking tests can be prepared by raining (pluviation) at the top of last laminar and distributed consistently. Sand raining technique can provide relatively homogenous specimens. In addition, it is simple, flexible and can be employed for preparing large or small samples with equal effectiveness (Eid, 1987). Therefore, the raining method was used for preparing the sand samples. To form a sand profile with uniform density, two factors are of prime importance in this method: (i) the height of fall of the sand and (ii) the intensity of deposition (i.e., the weight deposited per unit area). Varying either of these parameters controls the density of the sand as placed. The sand sample was placed by a raining container which was adjusted at constant height to distribute the soil accordingly (Figure 3.19). The uniformity of the soil samples was checked by monitoring the average relative density of the soil and monitoring the soil settlement at every shaking level (i.e., recorded weight of soil sample). 


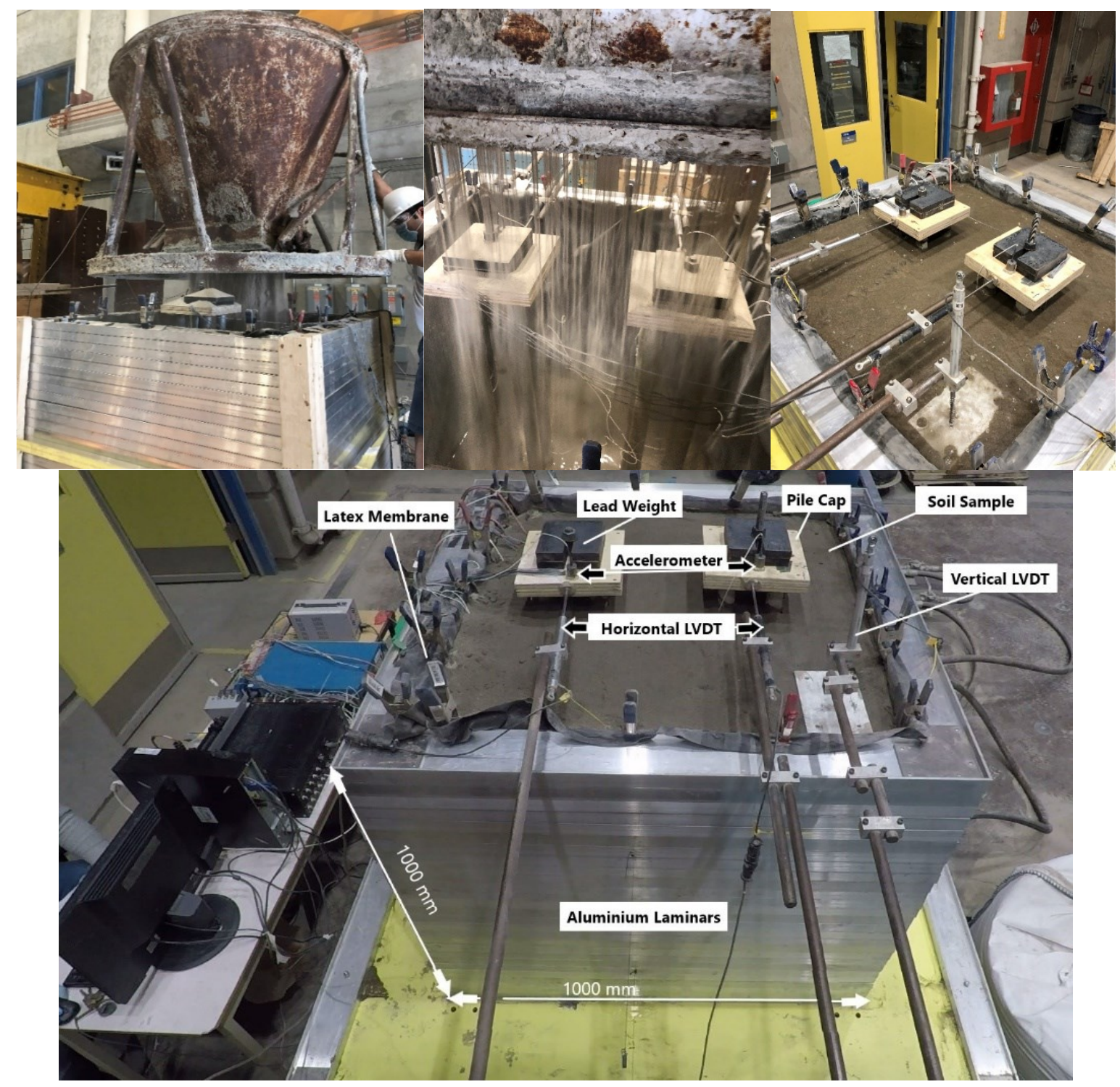

Figure 3.19: (a) Sand pluviation technique (left); and completion of sand pluviation and initiation for first excitation (right) (b)Various components of the shaking table tests in liquefiable soil

\subsection{Earthquake Input Motions}

The input ground motions in this study are represented by a set of real earthquakes recorded during the 2010 Ottawa earthquake (i.e., Val-des-Bois, Quebec) and the 1995 Kobe earthquake. The Ottawa earthquake from the Val-des-Bois region is probably the strongest earthquake that the city has felt in the past 200 years. Despite this, the estimated shaking from the recorded ground motions 
for Ottawa are well below the design level of shaking used in the 2005 National Building Code of Canada. For one or two storey structures the shaking from the Val-des-Bois event is about one fifth the design shaking. Ma and Motazedian (2012) stated that the earthquake threat to the two large cities of Ottawa and Montreal comes mainly from the Western Quebec Seismic Zone (WQSZ). The consequences of a significant earthquake near a major population centre in eastern Canada, such as Montreal, Ottawa, or Quebec City could be potentially disastrous today in terms of social and economic impact. An accurate and reliable assessment of seismic hazard is an essential prerequisite for effective mitigation measures in eastern Canada. The other real strong earthquake with extensive damages used here was the 1995 Kobe earthquake recordings of the Port Island downhole array $-79 m$ north-east component.

Each shaking model test was subjected to six excitation events at intensity levels of 50\%, $100 \%$, and $200 \%$ of the 2010 Ottawa event and $5 \%, 10 \%$ and $20 \%$ of the 1995 Kobe event. Three low level accelerations with a peak horizontal acceleration (PHA) of 0.02 to $0.08 \mathrm{~g}$ were targeted to ensure that the response remained in the elastic range. Three mid-range to high-range signals with a PHA of about 0.04-0.16g were applied to impart intermediate to high level excitations and induce nonlinear site and pile response. The initial shaking began with the low-level Ottawa seismic data at $50 \%$ intensity with a peak ground acceleration (PHA) of about $0.02 \mathrm{~g}$, followed by a mid-range signal at $100 \%$ PHA and, subsequently, a stronger shaking record at $200 \%$ level. A similar procedure was followed to apply scaled seismic data of the Kobe 1995 earthquake with an initial excitation of about $0.04 \mathrm{~g}$ (K5), followed by a mid-range PHA value at about $0.08 \mathrm{~g}$ (K10) and, finally, the strong shake level with a larger PHA of about $0.16 \mathrm{~g}$ (K20) in order to induce nonlinear soil and pile response (Figure 3.20 and 3.21). 

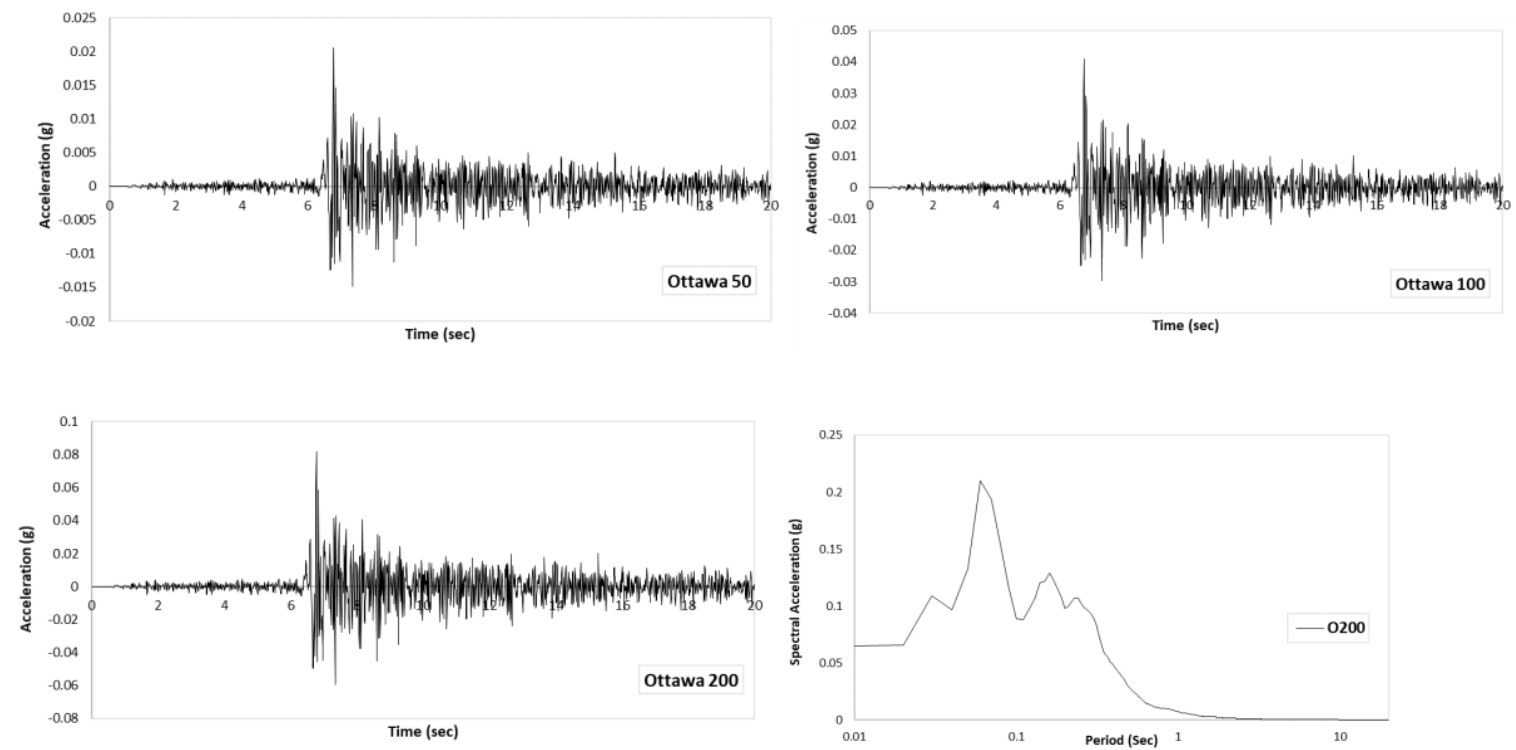

Figure 3.20: Scaled shaking events adopted in the shaking table experimental tests: acceleration time histories and response spectra at 5\% damping for 2010 Ottawa earthquake at Val-des-Bois in Province of Quebec
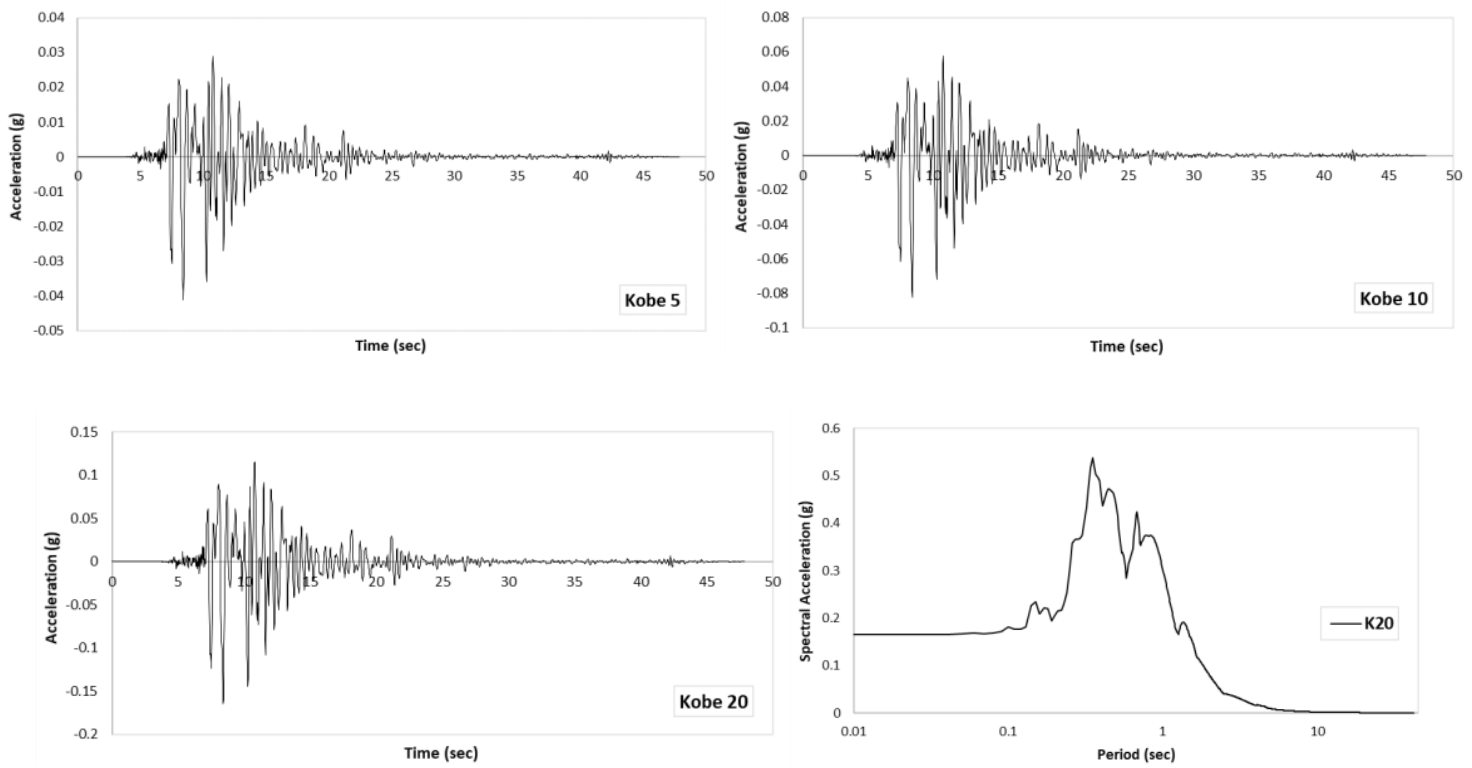

Figure 3.21: Scaled shaking events adopted in the shaking table experimental tests: three acceleration time histories at different intensity, and response spectra at 5\% damping for Kobe Earthquake, at Takatori recording station

\subsubsection{Data Analysis}

Upon completion of each phase, due to sensitivity of accelerometers caused by fluctuations in electric signal and often display high-frequency noise; filtering of raw data was performed to 
remove the noise from accelerometers readings employing Butterworth filter. Additionally, the adopted filtering for recorded data procedure was achieved by performing a $50 \mathrm{~Hz}$ low-pass filter routine called Fast Fourier Transform technique. The reason for selecting $50 \mathrm{~Hz}$ as an upper bond is to comply with the stated characteristics of the Carleton University-shaking table, where the frequency range of the generated input motion by shaking table is between 0-50 Hz.

\subsection{Shaking Table Testing Plan}

In this section, the shaking tests plan and preparation steps developed to complete this research were explained in detail which can provide better understanding of different aspects of this study. As stated, a laminar soil container is used to minimize the boundary effects and conduct 1-g shaking tests to simulate the free field soil behaviour, and the response of innovative hollow FRP piles (i.e., carbon or glass) and aluminium piles with enhanced physical modelling techniques. The shaking table testing plan was categorized to totally four phases which includes a total of 8 shaking tests to evaluate these model piles in two different mediums of liquefiable sand and soft clay. As discussed, model piles are divided into two groups of the end-bearing and frictional piles foundation with similar property and geometry with only variation in pile length. Each set is assembled of four model piles made of CFRP, four made of GFRP, and another four piles made of aluminium pipe piles with a diameter of $55 \mathrm{~mm}$, and length of $1000 \mathrm{~mm}$ and $750 \mathrm{~mm}$ for endbearing and friction piles, respectively.

In next chapters, the outcome of all four phases of this study is evaluated in terms of the pile type (i.e., end-bearing group piles and frictional groups piles) and pile materials (i.e., CFRP, GFRP, aluminium) in either uniform liquefiable soil and/or synthetic clay under constant load. Each chapter is written in journal paper format. The following chapter presents shaking table test 
results of end-bearing FRP group piles (i.e., carbon and glass) embedded in liquefiable soils. Followed by Chapter 5 which illustrates the shaking tests analysis of frictional FRP group piles in cohesionless soils (CFRP, GFRP and aluminium). This chapter also presents the amplification of ground motion and the seismic behaviour of frictional piles under shaking tests in cohesionless soil. Lastly, Chapters 6 and 7 present results and data analysis of shaking table tests on both frictional and end-bearing FRP and aluminium piles in cohesive soils. Finally, Chapter 8 provides a summary of the research outcomes along with recommendations for potential future research. 


\section{Chapter 4: Seismic Response of End-Bearing Fibre-Reinforced Polymer (FRP) Piles in Cohesionless Soils}

\subsection{Introduction}

Pile foundations are traditionally made of concrete, steel, and timber; however, there has been a great demand to explore innovative pile design using materials that can be more durable compared to conventional materials especially for harsh and corrosive environments. From 1987, several types of composite piles have been developed including reinforced plastic piles, concrete filled Fibre-Reinforced Polymer (FRP) piles, hollow FRP piles, and FRP sheet piles (Guades et al., 2012). The FRP is a composite material made of polymer fibers and an epoxy matrix. The most commonly used fibers in FRP piles are Carbon and Glass. While FRP materials may cause some complications associated with pile driving and high damping, they can be a suitable alternative in aggressive corrosive environments. These materials have been used in many infrastructure projects and applications in the last two decades (Kim, 2019). As a pile material, concrete filled FRP tubes have been proven to exhibit better load carrying capacity than conventional concrete piles (Mirmiran and Shahawy, 1996). These piles were also used as a substitute to precast concrete piles

for Route 40 highway bridge over Nottaway River in Virginia (Fam et al. 2003). The lucrative capabilities of FRP composite piles encourage further investigation on performance of these piles under both static and seismic loads.

Most past studies on FRP piles were focused on the load transfer and their flexural response under static loading (e.g., Iskander et al., 2001; Sakr et al., 2007; Hosseini and Rayhani, 2017). Helmi et al. (2006) studied the influence of pile driving forces and high-cycle fatigue on flexural performance of concrete-filled glass/carbon FRP piles and reported that driving forces may have 
minor influence on the flexural strength of CFRP piles. These piles were also reported to have higher deflections compared to pre-stressed concrete piles. However, FRP tubes needed larger driving energy and showed lower flexural resistance; therefore, the risk of buckling seems to be higher (Sakr et al., 2007). Hollow FRP piles were also examined under vertical and lateral static loads and showed higher axial load carrying capacity compared to similar steel pipe piles, while the flexural rigidity of the FRP piles were found to be less than the steel counterparts (Giraldo and Rayhani, 2014). Such high flexibility might provide a better performance under seismic loading in liquefiable soils.

Earthquake-induced liquefaction has caused considerable damage to pile foundations in many past earthquakes (e.g., the 1964 Niigata Earthquake and the 1974 Alaska Earthquake) which in turn resulted in damage to superstructures including bridges, port facilities, buildings, and utilities. Post-earthquake observations revealed that damage to pile foundations often occurred at depths rather than the pile heads, particularly near the interface between liquefied and nonliquefied soils (Meymand, 1998). The interaction between soil and pile foundations was also found to have a major impact on structures response under seismic loads (e.g., the 1995 Kobe Earthquake). The soil-pile interaction (SPI) in liquefiable ground is an extremely complex process involving inertial interaction between the superstructure and pile foundations, kinematic interaction between the piles and soil, seismically induced excess pore-water pressures, and the non-linear response of soils during strong earthquake motions (Finn, 2005). Gazetas and Mylonakis (1998) reported that period lengthening due to soil-foundation interaction can lead to an increase in structural forces during the earthquake. Site amplification and changes in foundation input motions resulted from the soil-pile interaction could also lead to foundation damage and collapse of structures. 
In the past few decades, several field and laboratory experiments were conducted to investigate the seismic interaction between soil and pile foundations in different site conditions (e.g., Matlock, 1962; Luco et al., 1988; Scott et al., 1982; Capattia et al., 2018; Wilson, 1998; Mason et al., 2013; Martakis et al., 2017; Stewart 1999). Shaking table tests in 1-g environment and centrifuge models have also been used to investigate the seismic response of model piles in wide range of soil conditions (e.g., Meymand, 1998; Lu et al., 2004; Chau et al., 2009; Hokmabadi et al., 2014; Unsever et al., 2016). Although, there is a rich literature on seismic performance of conventional piles using field testing, centrifuge model tests and shaking table tests, however, there is a lack of detailed information on seismic response of FRP composite piles. This paper presents results of a series of shaking table tests conducted on end- bearing hollow FRP pile groups made of carbon and glass fibres compared to conventional aluminium group piles. This chapter presents the conducted shaking table tests of the first phase of this study which include the seismic response of end-bearing model piles embedded within sandy soil in laminar container.

\subsection{Experimental Results and Discussion}

\subsubsection{Acceleration Time Histories}

Figure 4.1 illustrates a schematic configuration of all model instruments including accelerometers with assigned subscription (An), followed by Figure 4.2 shows sample acceleration times histories for the free field motion (A4) and corresponding pile motions for the CFRP (A5) and the conventional aluminium pile (A6) during the Kobe 20 event. As shown in previous schematic drawing, A2 and A3 are accelerometers attached to a pile per group near the base; A5 and A6 are mounted on the same pile at a depth close to the surface; A1 and A4 are located inside the soil at the same levels as A2 and A5 to record free field motions in the soil (far from the piles); A7 and 
A8 are mounted on the structure. The peak accelerations in terms of ' $\mathrm{g}$ ' at the accelerometer locations for free field motions as well as both the pile groups are presented in Table 4.1. From the time histories of acceleration, both the free field motions and accelerations recorded on pile foundations have significantly changes along the soil profile from the base to surface. The peak acceleration within the soil profile showed a small amplification from the base excitation to A1 and A4 along the soil profile for weaker seismic events (e.g., O50 and O100). As the input acceleration at the base of the soil model increased, the free filed motion at both the soil levels (A1 and A4) showed a decreasing trend (de-amplification). As it will be discussed later, this reduction in free field motion could be related to higher strain induced in the soil and the soil nonlinear response during the stronger input motions. However, the recorded peak accelerations within the soil (free field) for all applied excitations were significantly less than those measured on piles at the same depths. This difference in peak ground motions underscores the importance of considering soil-pile interaction impacts on foundation input motions in seismic prone areas. The difference between the free field and pile motions were significantly lower for the CFRP and GFRP pile groups compared to the conventional aluminium pile group. This behaviour is also attributed to the lower flexural stiffness (EI) and higher flexibility of the CFRP and GFRP piles, which leads to lower motions and closer acceleration level to those measured in the soil (free field motion). 


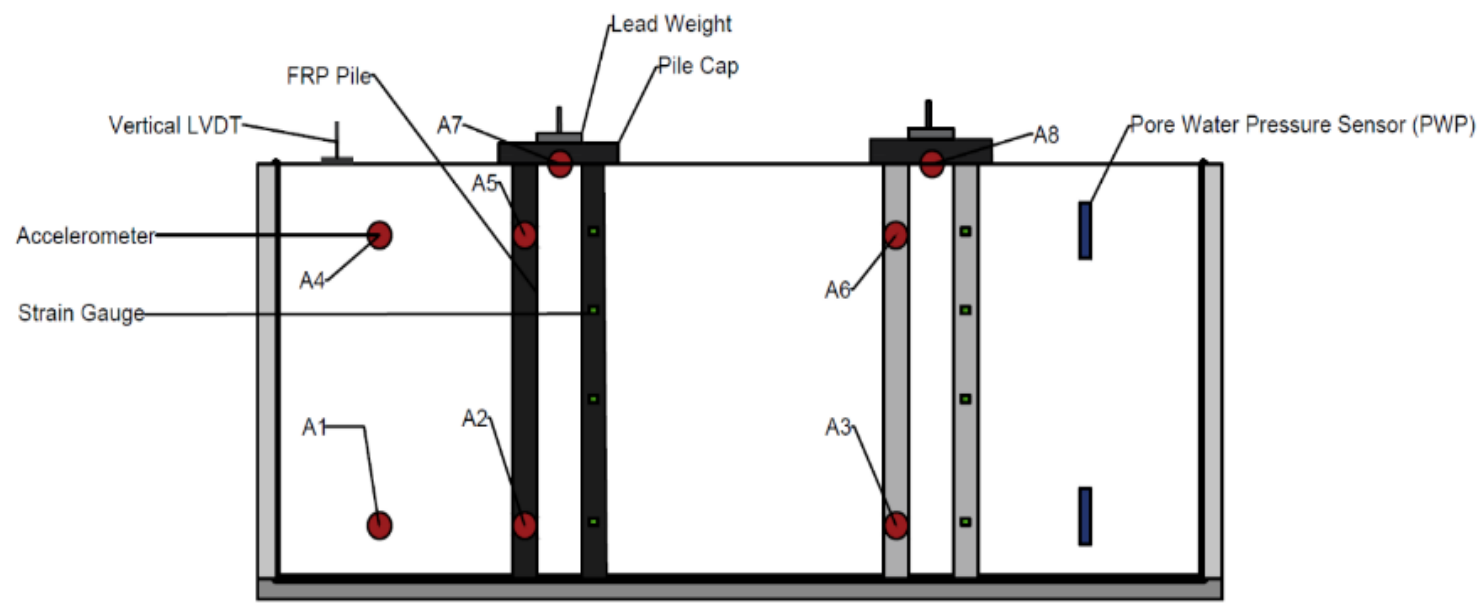

Figure 4.1: Schematic drawing of instruments used in model test; Accelerometer $\left(A_{n}\right)$; LVDT, Strain gauges and PWP sensors
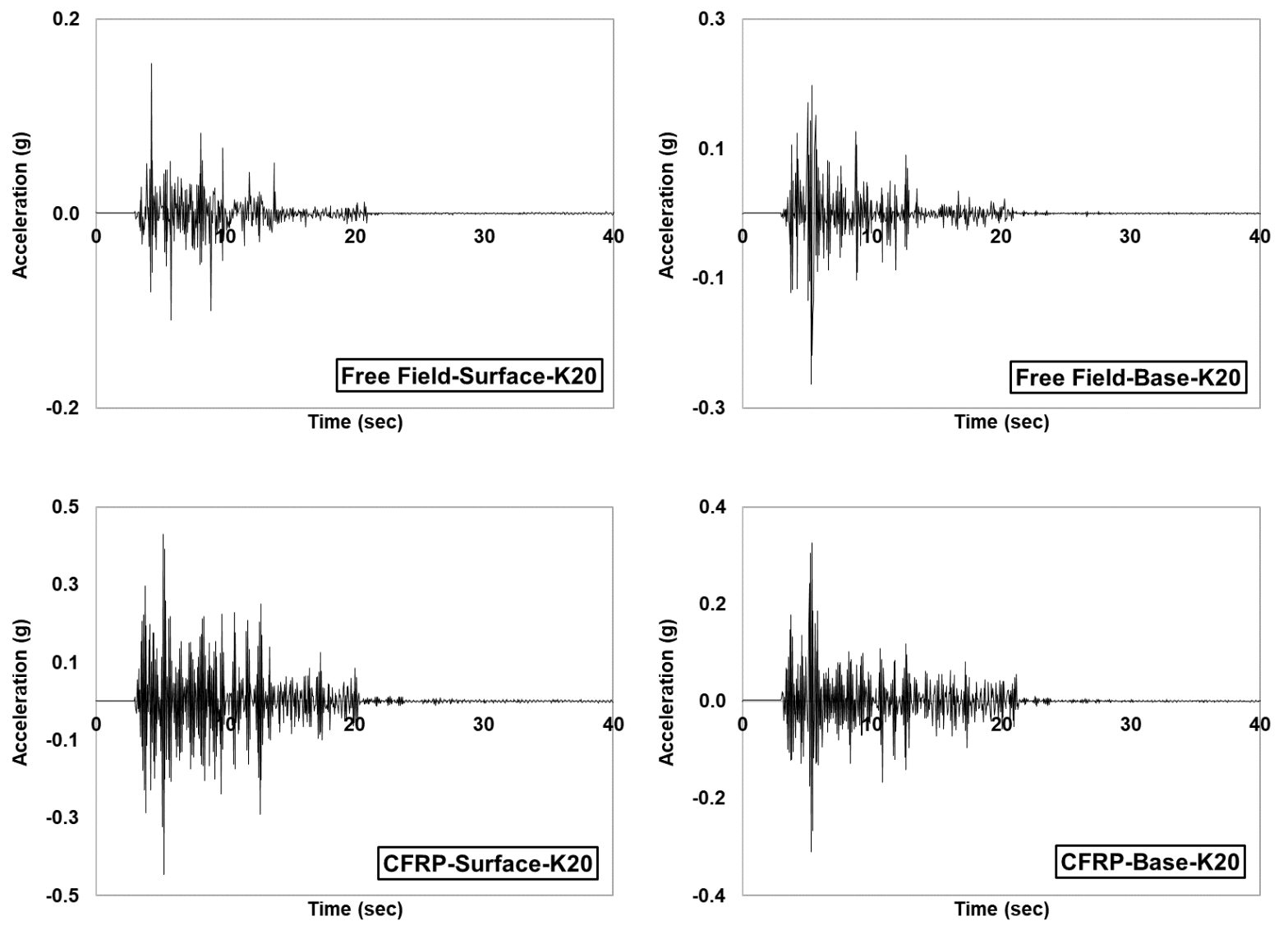

Figure 4.2: Acceleration time histories for the CFRP and Free Field at $200 \mathrm{~mm}$ depth during the Kobe 20 shaking event 
The distribution of acceleration varies along the shaft of all model piles. The FRP piles experienced smaller acceleration close to their base, followed by a rise in peak acceleration near the surface in all shaking events. However, the rate of increase in acceleration along the pile shaft was significantly higher for the aluminium piles and their pile cap experienced higher acceleration compared to both the CFRP/GFRP group pile caps. This also means that superstructures rested on aluminium group piles would have experienced significantly greater acceleration compared to structures sitting over FRP piles. This can be related to the higher stiffness of conventional piles (i.e., aluminium) and hence higher deviation between the foundation and free field motions resulted from kinematic soil-pile interaction. The higher peak accelerations in the aluminium pile cap may also result in generation of the base shear and induce moments that consequently can appear as displacement and rotation of the foundation relative to the free field.

Table 4.1: Peak accelerations at free field, pile cap and along the shafts for all events

\begin{tabular}{|c|c|c|c|c|c|c|c|c|c|c|}
\hline $\begin{array}{l}\text { Model } \\
\text { Piles }\end{array}$ & $\begin{array}{l}\text { Input } \\
\text { events }\end{array}$ & $\begin{array}{l}\text { Input } \\
\text { motions } \\
\text { (g) }\end{array}$ & $\begin{array}{l}\text { A1 } \\
\text { (g) }\end{array}$ & $\begin{array}{l}\text { A2 } \\
\text { (g) }\end{array}$ & $\begin{array}{l}\text { A3 } \\
\text { (g) }\end{array}$ & $\begin{array}{l}\text { A4 } \\
\text { (g) }\end{array}$ & $\begin{array}{l}\text { A5 } \\
\text { (g) }\end{array}$ & $\begin{array}{l}\text { A6 } \\
\text { (g) }\end{array}$ & $\begin{array}{l}\text { A7 } \\
\text { (g) }\end{array}$ & $\begin{array}{l}\mathbf{A 8} \\
\text { (g) }\end{array}$ \\
\hline \multirow{6}{*}{$\begin{array}{c}\text { CFRP } \\
\text { and AL } \\
\text { (T2) }\end{array}$} & $\mathbf{0 5 0}$ & 0.020 & 0.021 & 0.051 & 0.050 & 0.028 & 0.078 & 0.081 & 0.046 & 0.071 \\
\hline & 0100 & 0.041 & 0.040 & 0.081 & 0.094 & 0.039 & 0.115 & 0.122 & 0.101 & 0.117 \\
\hline & O200 & 0.082 & 0.103 & 0.123 & 0.159 & 0.098 & 0.158 & 0.169 & 0.118 & 0.157 \\
\hline & K5 & 0.041 & 0.076 & 0.121 & 0.112 & 0.049 & 0.146 & 0.139 & 0.128 & 0.131 \\
\hline & K10 & 0.082 & 0.108 & 0.218 & 0.129 & 0.095 & 0.205 & 0.227 & 0.168 & 0.171 \\
\hline & K20 & 0.164 & 0.267 & 0.327 & 0.297 & 0.217 & 0.450 & 0.498 & 0.301 & 0.501 \\
\hline \multirow{6}{*}{$\begin{array}{c}\text { GFRP } \\
\text { and AL } \\
\text { (T1) }\end{array}$} & O50 & 0.020 & 0.028 & 0.052 & 0.056 & 0.024 & 0.082 & 0.087 & 0.036 & 0.070 \\
\hline & 0100 & 0.041 & 0.045 & 0.072 & 0.087 & 0.046 & 0.122 & 0.136 & 0.107 & 0.131 \\
\hline & $\mathbf{0 2 0 0}$ & 0.082 & 0.098 & 0.209 & 0.289 & 0.096 & 0.225 & 0.249 & 0.153 & 0.224 \\
\hline & K5 & 0.041 & 0.071 & 0.126 & 0.127 & 0.058 & 0.134 & 0.158 & 0.118 & 0.123 \\
\hline & K10 & 0.082 & 0.112 & 0.176 & 0.187 & 0.077 & 0.213 & 0.231 & 0.153 & 0.178 \\
\hline & K20 & 0.164 & 0.261 & 0.254 & 0.330 & 0.155 & 0.405 & 0.474 & 0.310 & 0.523 \\
\hline
\end{tabular}




\subsection{Site Effect and Soil Amplification}

Spectral analysis was used to describe the frequency content of earthquake motions measured on model piles and within the soil. Figure 4.3 demonstrates free field spectral response of the soil in comparison with the base input motions during the Kobe 5 and Kobe 20 events. In general, the input motion decreases as the seismic waves propagates along the soil profile, and the free field spectral acceleration at the top of the soil model is significantly lower than the base input motion in the period range higher than $0.08 \mathrm{sec}$. (frequency of $0.2-10 \mathrm{~Hz}$ ). As the K20 event is a strong event with a base excitation of $0.16 \mathrm{~g}$, this de-amplification would be due to higher levels of nonlinearity and possibly related to liquefaction induced strains in the soil. However, a small amplification was recorded at low period range of $(0.02-0.03 \mathrm{sec})$ corresponding to a high frequency motion of about 30-50 Hz for the K20 event.
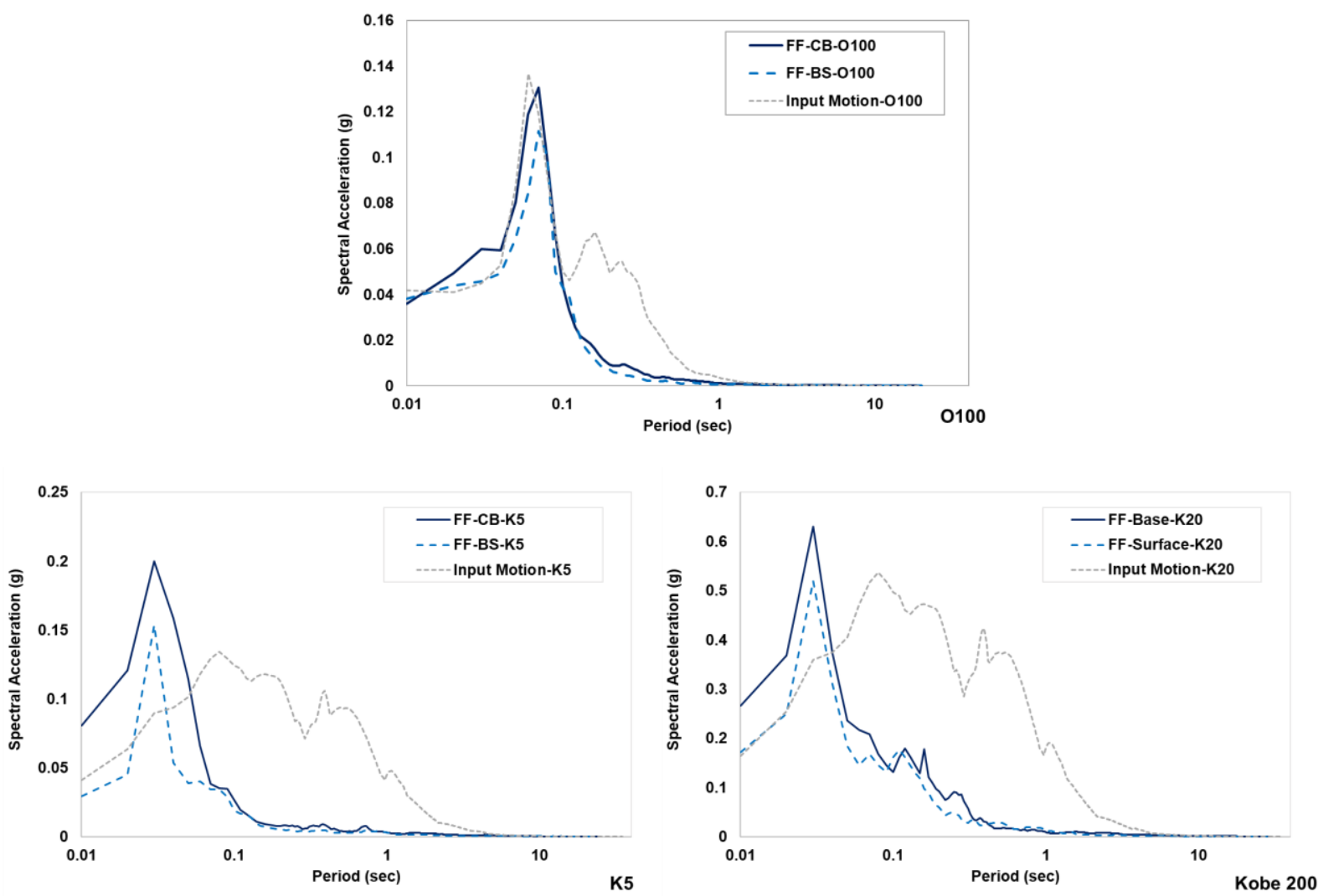

Figure 4.3: Response spectra for free field during the O100, K5 and K20 excitations 


\subsection{Effect of Soil-Pile Interaction (SPI) on FRP Piles}

Previous studies have shown that the kinematic soil-structure interaction could deviate the foundation input motions from the free field motions and, hence, affect seismic response of superstructures. Consequently, this may cause foundation rocking due to base-slab averaging and embedment effects (Stewart et al., 1999). This interaction can be defined as frequency dependant transfer function involving both the free field motion and the base-slab motion while the base and structure are assumed massless. To examine the influence of kinematic soil-pile interaction on foundation input motions, the spectral acceleration closer to ground surface for all the model piles were compared with the spectral response of the free field motions at the same depth. A pile/soil response spectral ratio (RRS) was defined to investigate the impact of kinematic interaction on pile foundation motions. This spectral ratio is plotted for all the model piles measured at the Kobe (K20) event in Figure 4.9 as an example. The spectral acceleration recorded on all model piles were shown to be higher than those recorded at the same level within the soil (free field motion). The ratio of foundation to free field motions were significantly higher at the frequency range of 1$20 \mathrm{~Hz}$. The period of vibration also showed to be higher for the foundation motions compared to the free field records. This increase in spectral acceleration for the pile models and change in frequency content of the seismic waves are clearly associated with the difference in stiffness of the pile materials compared to that of the soil and, hence, kinematic interaction between the model piles and soil. A similar comparison can be made for the different model piles to evaluate the impact of pile materials on the pile-soil interaction and, in turn, the seismic response of different foundation systems. As illustrated in Figure 4.4, the aluminium piles demonstrate similar behaviour compared to the model FRP piles at mid-range excitation of O100, especially at the frequency range of 5-20 Hz. However, higher RRS values were observed for the CFRP piles close 
to the base compared to the aluminium piles at stronger excitation of K20, at frequency range of 1.0-20 Hz, which could be an indication of variation in the soil-pile interaction between FRP and conventional materials.

Additionally, considering the motion response for the two FRP materials (i.e., CFRP and GFRP), the CFRP piles experienced higher levels of acceleration and led to higher amplifications compared to the to the GFRP piles. Therefore, it seems that the response of CFRP piles could be an indication of more dominant kinematic interaction compared to the GFRP ones especially during the frequency of about $10 \mathrm{~Hz}$. This behaviour has eventually led to larger levels of amplification for the CFRP piles compared to the GFRP pile group. The difference in seismic response of these two pile groups could be related to the difference in rigidity and stiffness of different FRP materials. As stated earlier, the elastic modulus (E) of the CFRP materials (as an indicator of stiffness and rigidity) was higher than the glass fibers at around 7.0 GPa compared to the GFRP materials at 5.8 GPa. This difference in rigidity could have led to higher acceleration transferred through the CFRP piles and hence higher-level of amplification of foundation input motions. The frequency content of the amplified motions appears to be consistent for all model pile groups. Thus, the soil-pile interaction has not deviated the frequency content of seismic motions recorded for the pile systems and only amplified the input motion.
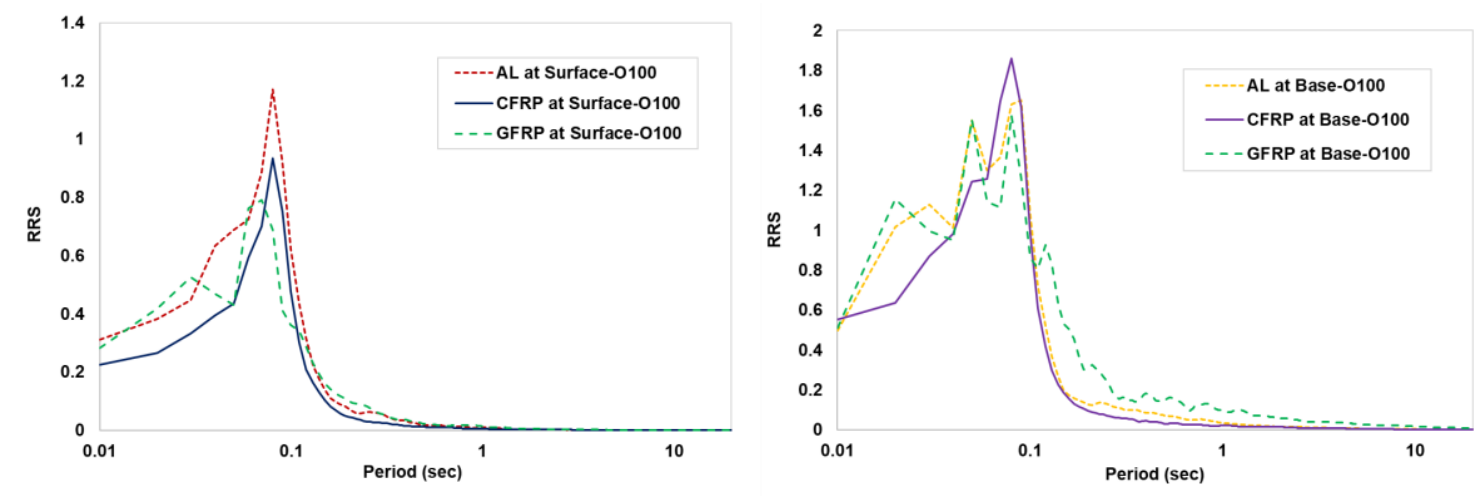

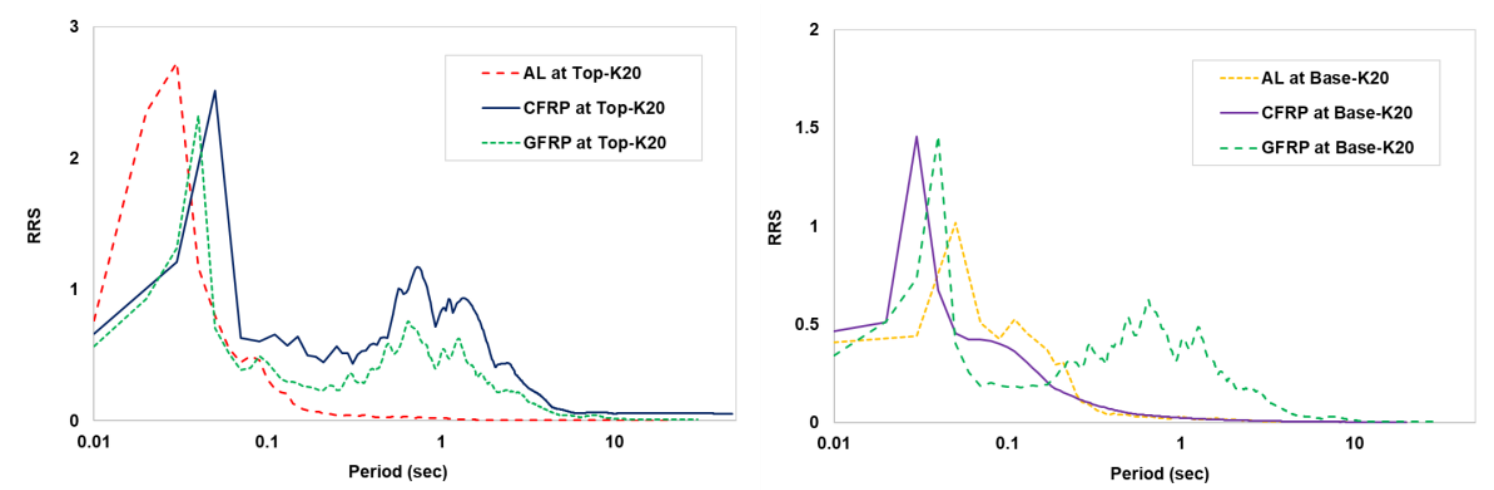

Figure 4.4: Ratio of response spectra (RRS) of pile models along the shaft during O100 and K20 shaking event

To further examine the influence of pile materials on seismic response of structures, the spectral response of the pile cap for all model piles are illustrated in Figure 4.5. As expected, the pile caps for both FRP model piles have experienced significantly lower accelerations than the pile cap for aluminium piles. This can be explained by the higher flexural stiffness and rigidity of aluminium piles compared to FRP piles. $\left(\mathrm{E}_{\mathrm{AL}}=7.8 \mathrm{GPa}\right.$; $\left.\mathrm{E}_{\mathrm{CFRP}}=7.0 \mathrm{GPa}\right)$. Pile made of composite FRP materials seems to provide higher flexibility along their shaft. Among the FRP piles, the pile cap for CFRP piles showed higher amplification compared to the one for GFRP piles which could correspond to higher flexibility of GFRP piles compared to the CFRP piles. Therefore, the GFRP piles could be a suitable composite FRP option for pile foundations in seismic prone area for loose and liquefiable soils due to their higher flexibility and hence lower amplification of foundation input motions. 

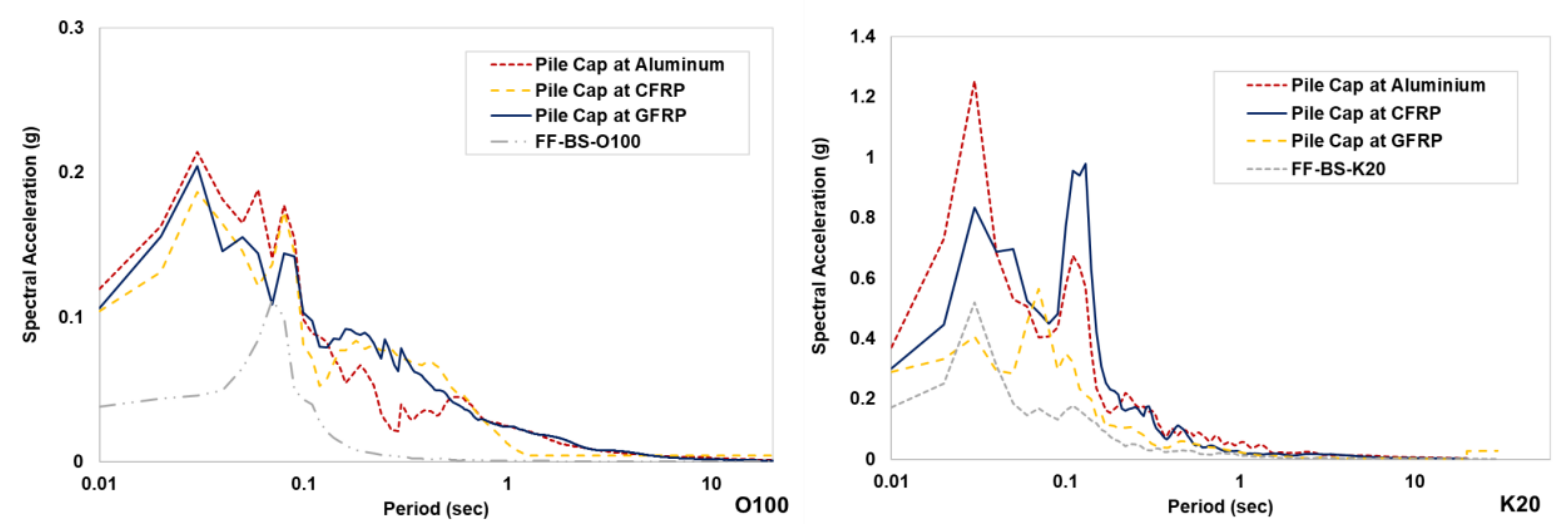

Figure 4.5: Pile cap reponse spectra at $\mathrm{O} 100$ and K20 shaking events for all model piles

\subsection{Pile Bending Moment}

A series of strain gauges were placed over model piles at different depths to record possible lateral strains of the piles during shakings and, in turn, estimate bending moment envelope for the model piles. The bending moment envelope is defined by the absolute peak strain at each gauge during the excitation. This means that the result is not equivalent to actual bending moment diagram at the time step when the peak strain is recorded. As stated by the theory of elasticity and Hooke's law (Timoshenko, 1940), the generated moment in the pile section is a function of the recorded strain in the strain gauges, referring to the following equation:

$$
M=\frac{2 E I}{D} \times \varepsilon
$$

where, $E$ is the modulus of elasticity, $I$ is defined as the cross sectional moment of inertia, $D$ is the outer diameter of the pile, and $\varepsilon$ is defined as the recorded strain in the strain gauges. Figure 4.6 illustrates change in the bending moment along the pile shaft for both the FRP pile groups and conventional pile (aluminum) at shaking event of $\mathrm{O} 100$ and K20. At shaking events of O100, the distribution of the moment amplitude along the FRP pile shafts demonstrate similar trends but 
magnitude may vary due to the variation in flexural stiffness EI. However, the pile response at K20 explains that CFRP model piles developed slightly greater bending moments than GFRP piles along the shaft. This means CFRP piles may have experienced larger kinematic forces which this behaviour can be also clearly noticed in the aluminium piles where very large bending moment was recorded at the toe. Alternatively, this increase in bending moments, especially close to the base, suggests that liquefaction-induced kinematic loads dominated the pile response (Meymand, 1998). Correspondingly, any change in bending moment beneath the surface is due to generation of inertial forces from the superstructure masses, which may induce larger bending moments near the pile heads. This outcome can be evidently seen in the CFRP and aluminium piles (i.e., beneath the surface).
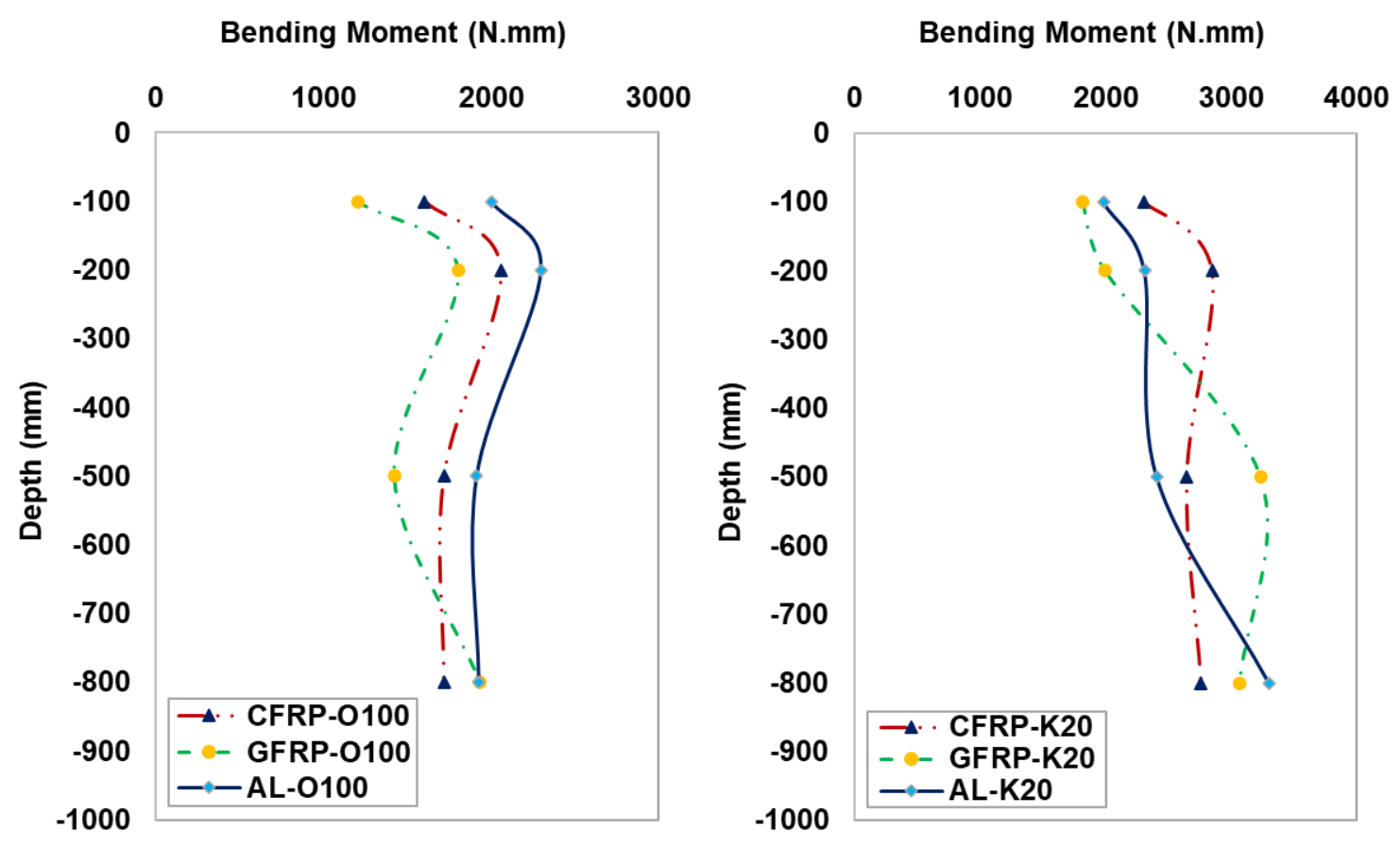

Figure 4.6: Recorded bending moment distribution along the piles at O100 and K20 
To further validate the interaction effects, Figure 4.12 shows lateral deflections of the pile caps for all model piles during the K20 shaking. The lateral deflections for the conventional aluminium pile cap was the highest among all model piles. These results also revealed significant lateral deflections for the CFRP pile cap at K20 compared to the GFRP pile head. This can additionally confirm that Carbon FRP piles have experienced higher kinematic forces which in turn induced larger bending moment and comparably, larger deflection at pile cap. This result can also further underscore the importance of pile ductility and hence the kinematic pile-soil interaction on seismic response of pile foundations in seismic prone areas.

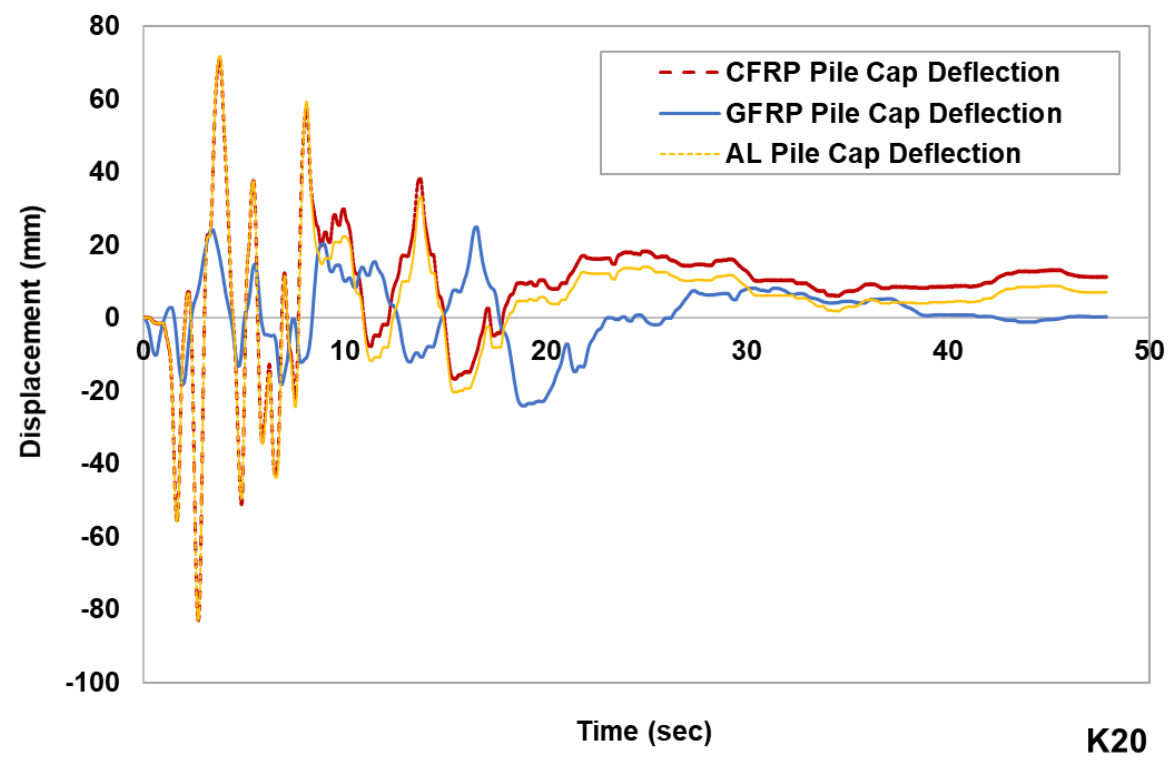

Figure 4.7: Pile head/cap deflection at the K20 event

\subsection{Impact of Liquefaction on Lateral Response of Pile Models}

During the shaking period, excess pore water pressures were monitored by piezometers at two different depths. Figure $4.8 \mathrm{a} \& \mathrm{~b}$ presents the PWP time history during the Kobe shakings at the base (CB) and at the Surface (BS), respectively. Each plot presents a significant change in PWP as the shaking levels of the earthquake events increase. The increase of pore water pressure implies 
that the water in pore voids attempt to flow out from the soil to areas with lower pressure such as ground surface. It is observed that the excess pore water pressure proceeded from the bottom up as the excitations are applied at the base of the soil models. The peak increase in pore water pressure measured at the base of the soil models was about $11 \mathrm{kPa}$, while the highest increase in pore water pressure closer to the soil surface was measured at about $0.85 \mathrm{kPa}$.

The excess pore water pressure ratio $\left(\mathrm{R}_{\mathrm{u}}\right)$ is defined as the ratio of the excess PWP $(\Delta u)$ over the initial effective stress $\left(\sigma_{o}{ }^{\prime}\right)$. This was a modest indicator to state the occurrence of liquefaction. Liquefaction is generally occurred if $R_{u} \geq 1$. However, if $R_{u}<1$, it is considered that no liquefaction arisen. It is important to notice that the larger $\mathrm{R}_{\mathrm{u}}$ values (i.e., $\mathrm{K} 10$ and $\mathrm{K} 20$ events) may potentially reveal that piezometers may have vertically dropped downward due to gravity and as a result of soil settlement over several excitations which this may slightly alter the measurement for PWP values. During Ottawa excitations as can be deduced from the trends in Figure 4.9, $\mathrm{R}_{\mathrm{u}}$ values did not reach the limit of 1.0 as weaker earthquake with lower acceleration amplitude was intended to monitor the FRP piles response as the rate of increase in generation of PWP can be noticed. This outcome can clarify that pile models may have not experienced large kinematic forces under weaker excitations (e.g., O50, and K5); therefore, insignificant variation of bending moment was achieved. Correspondingly, the change in PWP can be also correlated to settlement as this surface settlement was monitored vertically and measured at additional spots by manual reading after each shaking. The settlement was confirmed to be uniform across the soil surface as result of using the laminar boundary conditions (Figure 4.9). The result can be indicator of contractive soils behaviour (i.e., loose soils), which are subject to volume reduction or sudden settlement, are considered susceptible to liquefaction behaviour as observed in K10 and K20, as PWP increase, there is a decrease of the effective stress of such soil diminishes the contacts 
between their particles where this outcome can be resolution of full liquefaction that has consequently overshadowed the response of the pile under earthquake loading.

Furthermore, prior to initiating the first shaking, the initial average relative density was about $49.8 \%$ which indicated that the sand deposit can be categorized as loose sand. After first three shaking events (O50, O100, O200), the sand surface settled by about $3 \mathrm{~mm}, 4.5 \mathrm{~mm}$, and 5.5 $\mathrm{mm}$, respectively; as a result, the average relative density of the sand specimen reached to approximately 58\%. After execution of each excitation, the PWP ratio tended to decrease (dissipation of PWP), and the sand specimen had become denser and its liquefaction resistance would have increased. At fourth excitation (i.e., K5), the soil contractive behaviour can be evidently seen followed by quick increase in settlement. According to the $\mathrm{R}_{\mathrm{u}}$ value and settlement results, soil deposits reached full liquefaction at two excitation stages of K10 and K20 with stronger motions, as expected. This eventually caused significant settlement of about $10 \mathrm{~mm}$ and $38 \mathrm{~mm}$ (i.e., average of $\mathrm{T} 1$ and $\mathrm{T} 2$ ), respectively leading to immediate densification of soil with increase in relative density up to $80 \%$. Figure 4.10 presents liquefaction development and large quantity of sand boils that were developed at the surface and around the piles a few seconds after the last strong earthquake motion (K20). The occurrence of liquefaction was initiated during moderate shakings while complete liquefaction occurred under strong shaking. As expected in K20 motion, the value of peak excess PWP reached within the moments where the input peak acceleration was applied. This outcome may additionally explain the increase in bending moments toward deeper positions $(\mathrm{CB})$ on the model piles specifically the aluminium piles where the liquefaction-induced kinematic loads dominated the pile's response. Hence, the distribution of bending moments along the model piles' length was proportionally affected by interface of liquefied soil at K20 excitation where full liquefaction occurred, as expected in results of bending 
response of aluminium pile models at its base. CFRP piles, however, experienced larger peak bending moment near the surface. This behaviour may contribute to cumulative settlement due to several excitations that have resulted in increase of density. Therefore, CFRP as a very light pile member may not become dominated by kinematic interaction at its base thus this may exhibit to greater response and peak bending moment near the surface.
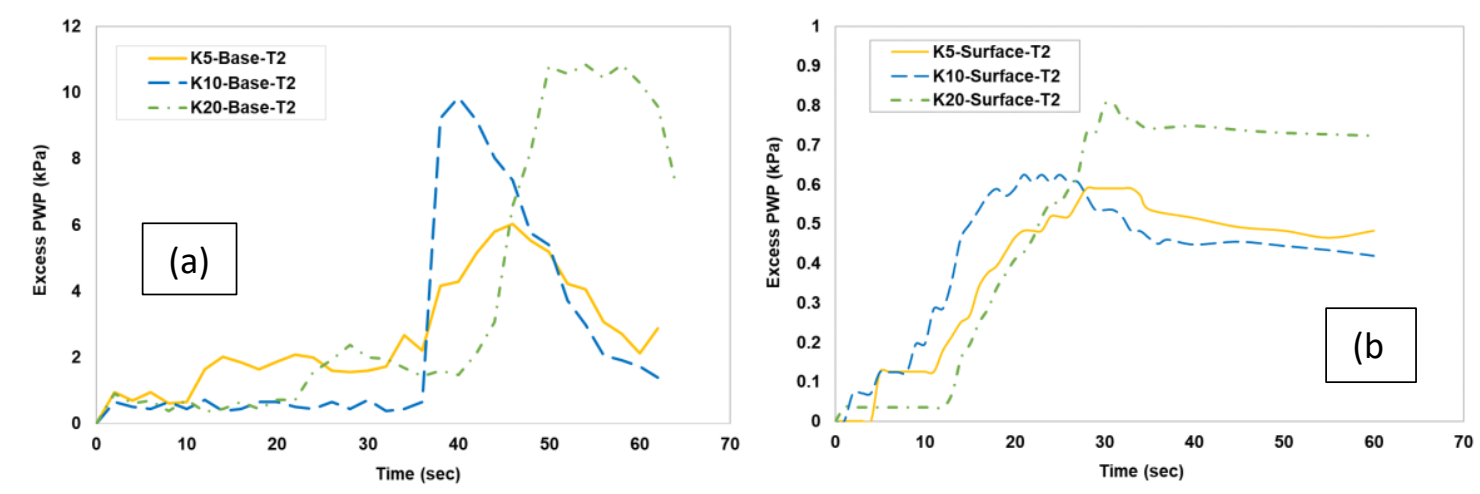

Figure 4.8: Excess pore water pressure during the Kobe shaking event- T2

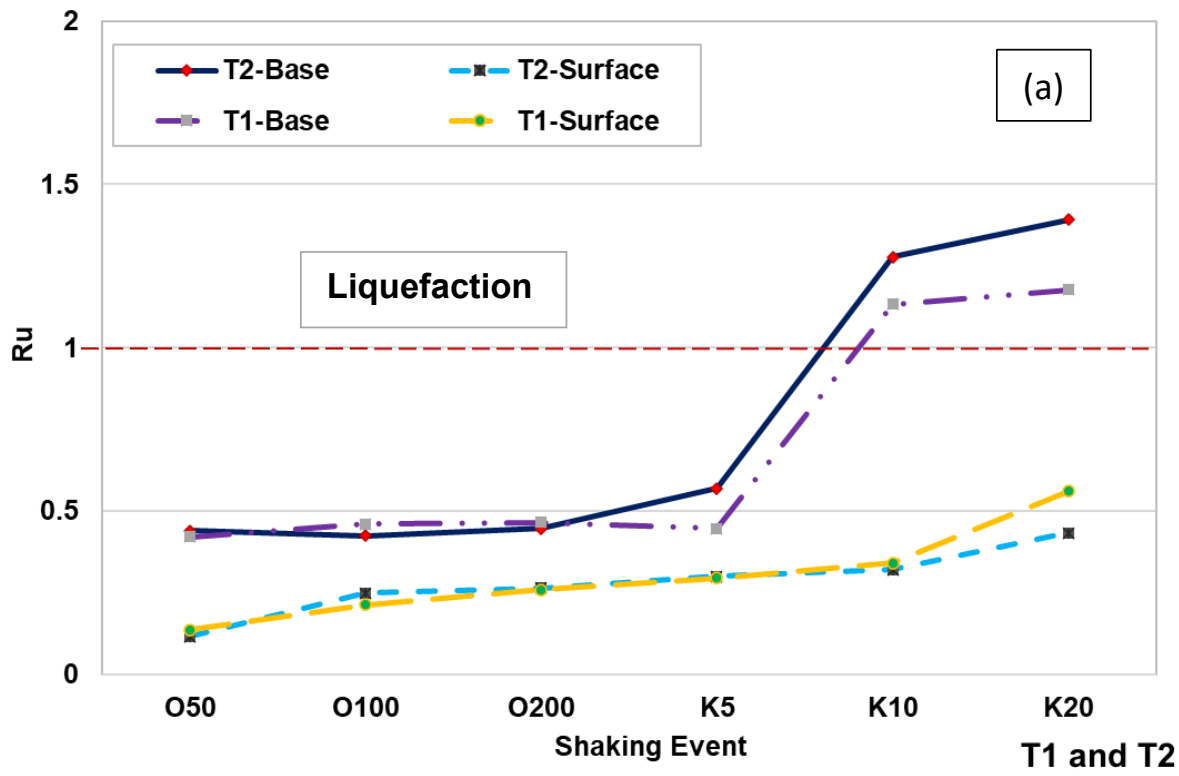




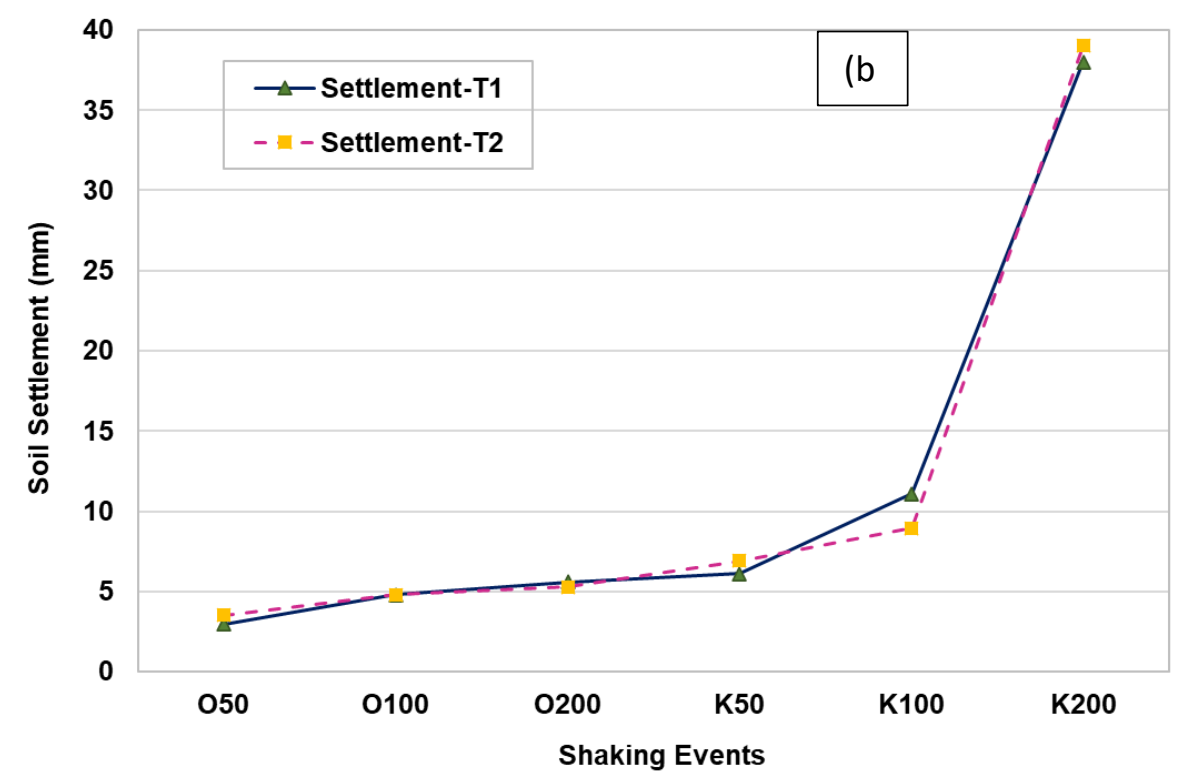

Figure 4.9: (a) Rate of pore water pressure; (b) soil settlement for all shaking events at T1 and T2

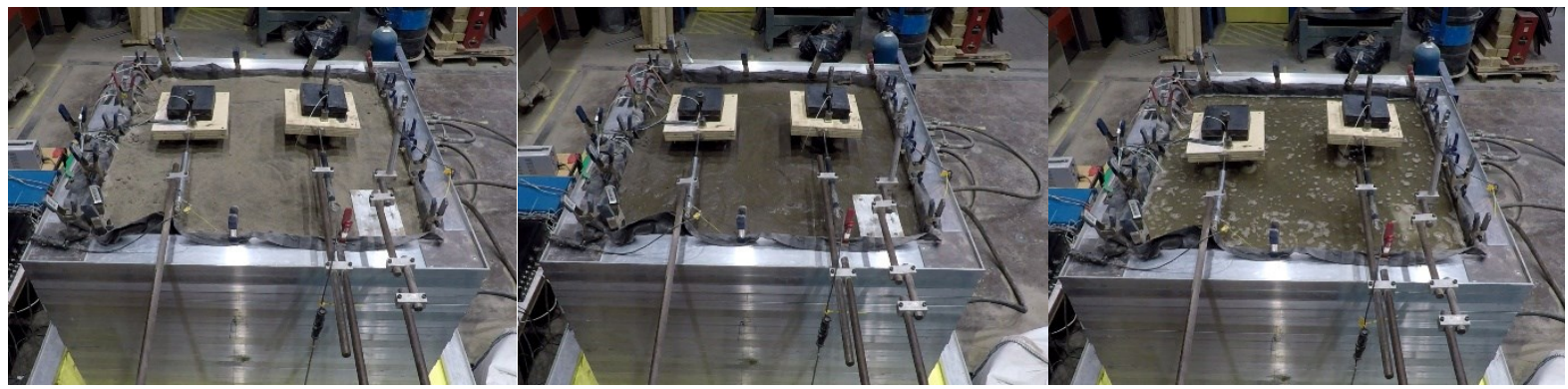

Figure 4.10: Development of sand boiling during the eaqrthquake loading (left to right)

\subsection{Summary}

Two series of shaking table tests were conducted on three different pile models made of conventional materials (i.e., alluminium) and two Fiber-reinforced Polymer materials (glass and carbon) embedded as end-bearing piles in fine sand. The shaking events used here were scaled acceleartions recorded during the 2010 Ottawa and the 1995 Kobe earthquakes.

The foundation motions recorded along the model piles showed significantly higher levels of excitation compared to the acclerations recorded within the soil at the same lavel. Most these amplifications were recorded at a period of $0.05-0.1$ seconds, corresponding to the frequency range 
of 10-20 Hz. This higher level of foundation motions recorded on the model piles were related to kinematic pile-soil interaction which deviated the foundation motions from that of the soil. The impact of such interaction was, however, different for each pile type. Foundation motions recorded on the aluminium piles as well as the pile cap on these piles were significantly higher than those recorded on FRP model piles. This was attributed to the higher rigidity and, hence, lower flexibility of conventional pile materials compared to the piles made of composite FRP materials.

Among FRP piles, the level of amplification of earthquake motions for glass FRP piles (GFRP) was shown to be lower than that for the carbon one (CFRP). This behaviour was also related to the difference in flexural stiffness of the two pile groups. Material characterization of the model piles revealed much higher flexibility for the GFRP piles, leading to lower recorded kinematic pile-soil interaction and, hence, lower foundation motions.

The frequency content of the amplified motions appeared to be consistent for both the model pile groups. Therefore, the soil-pile interaction has not deviated the frequency content of seismic motions recorded for both the conventional and FRP pile systems and only amplified the input motion. Furthermore, from the response spectra results, the CFRP and GFRP model piles showed similar frequency trend at the same level of earthquake intensity. 


\section{Chapter 5: Seismic Performance of Frictional FRP Piles in Sand}

\subsection{Introduction}

The behaviour of foundations during earthquakes is often dictated by the response of the supporting soil. In general, there are two problematic types of ground response during an earthquake: (a) liquefaction (e.g., the 1995 Kobe earthquake), and (b) amplification of the ground motion (e.g., the 1989 Loma Prieta earthquake and the 1985 Mexico earthquake). The soil-pilestructure interaction also plays an important role in seismic response of pile foundations and can cause considerable damage to pile foundations (e.g., the 1964 Niigata Earthquake and the 1974 Alaska Earthquake). Post-earthquake observations revealed that damage to pile foundations often occurred at depths rather than the pile heads, particularly near the interface between liquefied and non-liquefied soils (Meymand, 1998). The interaction between soil and pile foundations was also found to have a major impact on structures response under seismic loads (e.g., the 1995 Kobe Earthquake). The soil-pile interaction (SPI) in liquefiable ground is an extremely complex process involving many factors including inertial interaction between the superstructure and pile foundations, kinematic interaction between the piles and soil, seismically induced excess porewater pressures, and the non-linear response of soils during strong earthquake motions (Wilson, 1998; Fam, 2000; Gao et al., 2011; and Finn, 2005).

Recently, Fibre-Reinforced Polymer (FRP) materials have been used as an alternative in many applications such as piling industry. The current state-of-practice related to the engineering use of FRP piles indicates that FRP piles primarily have been used experimentally for fender piles, waterfront barriers, and bearing piles for light structures (Busel, 1995; Iskander and Stachula, 1999). In 1998, Hudson River Park with over 100,000 bearing piles was facing significant amount 
of damage due to corrosion of steel, deterioration of concrete, and vulnerability of timber piles to the marine environment, therefore, FRP materials with high degradation resistance, were suggested as an alternative pile material. Shao (2003) was able to achieve considerable ductility in concrete columns with glass FRP tubes as a replacement of the entire internal steel. Mirmiran et al. (1996) reported that the entire steel reinforcement in a concrete column or pile could be replaced by the FRP tube without affecting its load carrying capacity under static loading.

The engineering application of FRP specifically in geotechnical application may present competitive alternatives to conventional pile materials, due to the accelerated degradation of these conventional materials in aggressive environments. Moreover, there are many challenges and frustration in industry to repair and maintain conventional piles including applying chemical treatment of wood pilings (i.e., due to the toxic nature of these treatments). Kim (2019) states that several companies used FRP materials as alternative to different components including bridge girders, decks, piers (columns), piles, abutment, buried structures, concrete pavement, drains, and culvert liners. The actual application and its adaptation began in more construction project sometime in 2000s. As a result, the concept has been applied in several piling applications in the United States such as precast piles on Route 40 bridge in Virginia (Fam et al. 2003) and Liberty Statue Duck. Meanwhile, several studies have been conducted on load transfer and mechanical and flexural behaviour of FRP piles (Mirmiran et al., 2002; Mohamed and Masmoudi, 2010; Hosseini and Rayhani, 2017). The lucrative capabilities of FRP composite piles encourage further investigation on performance of these piles as there are limited studies on dynamic response of FRP materials. The goal of this study is to examine seismic performance of hollow FRP piles using a series of shaking model tests in liquefiable sand compared to traditional piles such as aluminium pile. This chapter presents results of shaking table tests on hollow FRP (carbon and glass) and 
traditional aluminium frictional group piles embedded within sandy soil in laminar container. The impact of pile type (frictional vs end-bearing) on seismic response of pile foundations are also discussed.

\subsection{Acceleration Time Histories}

Figure 5.1 illustrates a schematic configuration of all model instruments including accelerometers with assigned subscription (An); A2 and A3 are accelerometers attached to a pile per group near the base; A5 and A6 are mounted on the same pile at a depth close to the surface; and ultimately, A7 and A8 are mounted on the pile cap. Figure 5.2 demonstrates acceleration times histories for the free field motion at the base and near the surface (i.e., A1 and A4) and corresponding pile motions for the CFRP at the base (A2), and surface (A5) during the Kobe 20 event. Additionally, the peak accelerations in terms of ' $\mathrm{g}$ ' at the specified locations for free field motions as well as all the pile groups are presented in Table 5.1.

From the time histories of acceleration, both the free field motions and accelerations recorded on pile foundations have significantly changed along the soil profile from the base to surface. The peak acceleration within the soil profile showed a smaller amplification from the base excitation to A1 and A4 along the soil profile for weaker seismic events (e.g., O50 and O100). As the input acceleration at the base of the soil model increased, the free filed motion at both the soil levels (A1 and A4) showed a decreasing trend (de-amplification). As it will be discussed later, this reduction in free field motion could be related to higher strain induced in the soil and the soil nonlinear response during the stronger input motions. However, the recorded peak accelerations within the soil (free field) for all applied excitations were significantly less than those measured on piles at the same depths. This difference in peak ground motions underscores the importance of 
considering soil-pile interaction impacts on foundation input motions in seismic prone areas. The overall results of peak acceleration measured on piles' profile revealed relatively similar response for the CFRP and AL frictional pile groups, while the GFRP piles recorded lower amplitude of acceleration. This behaviour may be attributed to higher ductility of CFRP materials compared to CFRP and then aluminium pile material, leading to lower motions and closer acceleration level to those measured within the soil (free field motion).

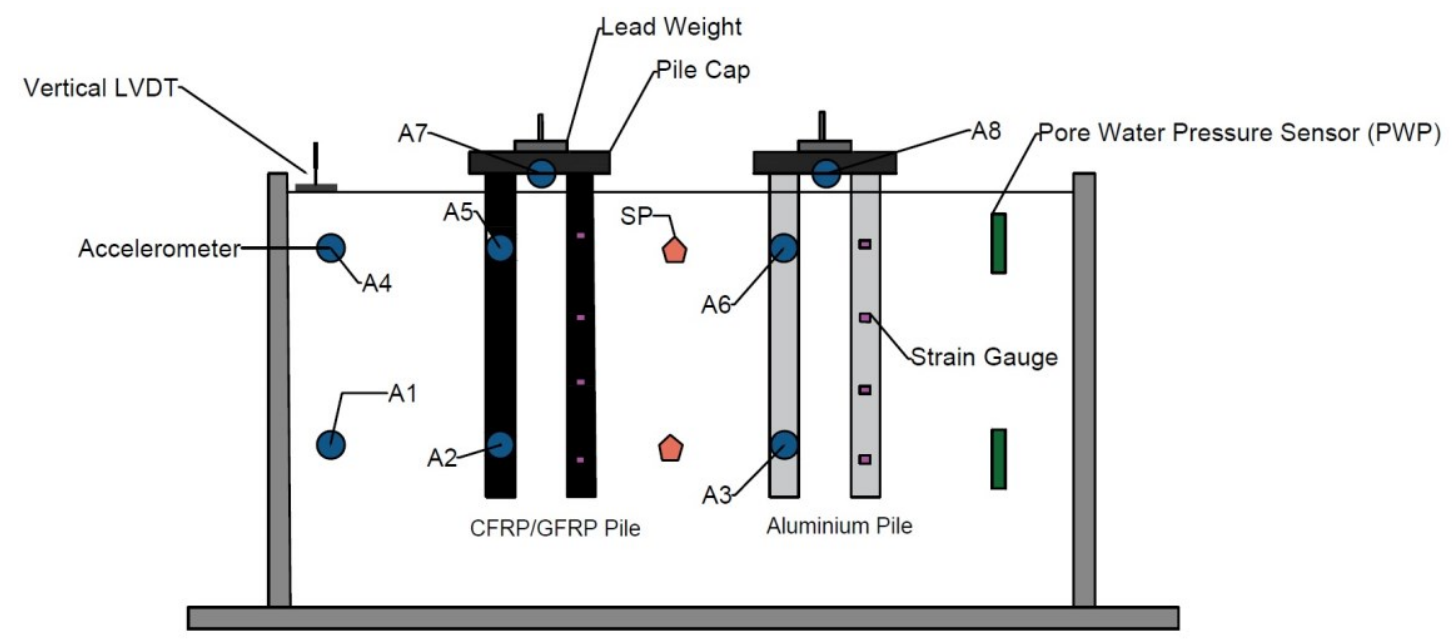

Figure 5.1: Schematic drawing of instruments used in model test; Accelerometer $\left(A_{n}\right)$; LVDT, Strain gauges and PWP sensors
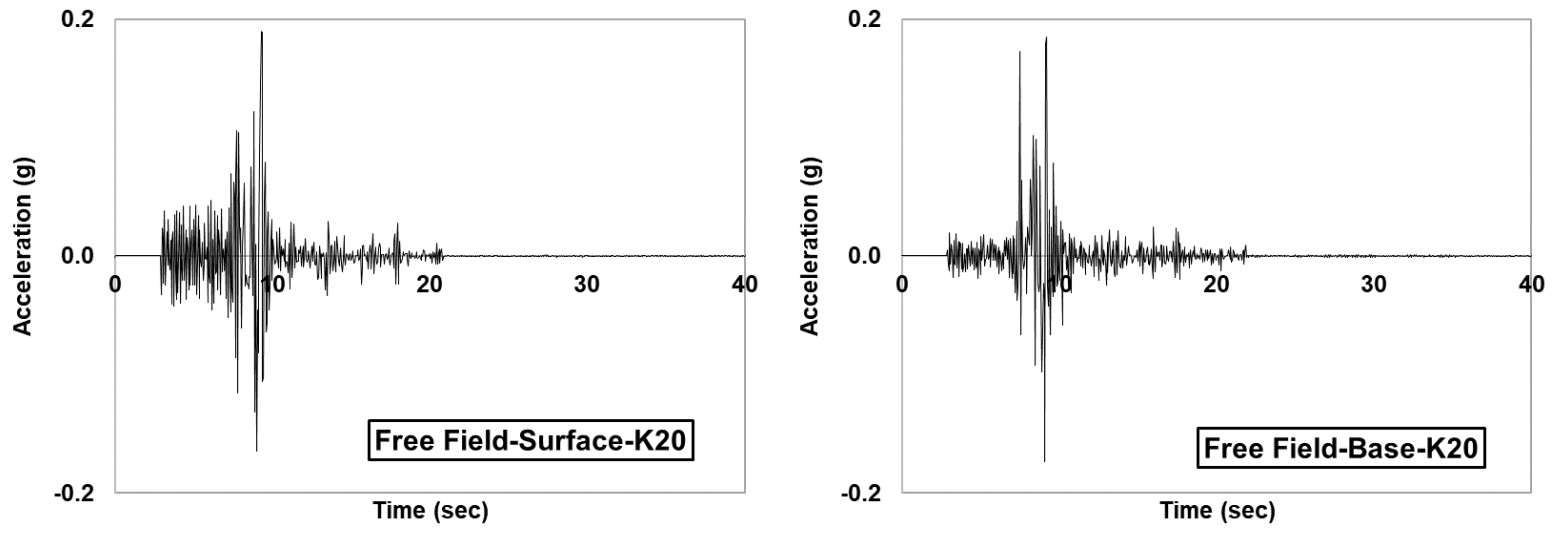

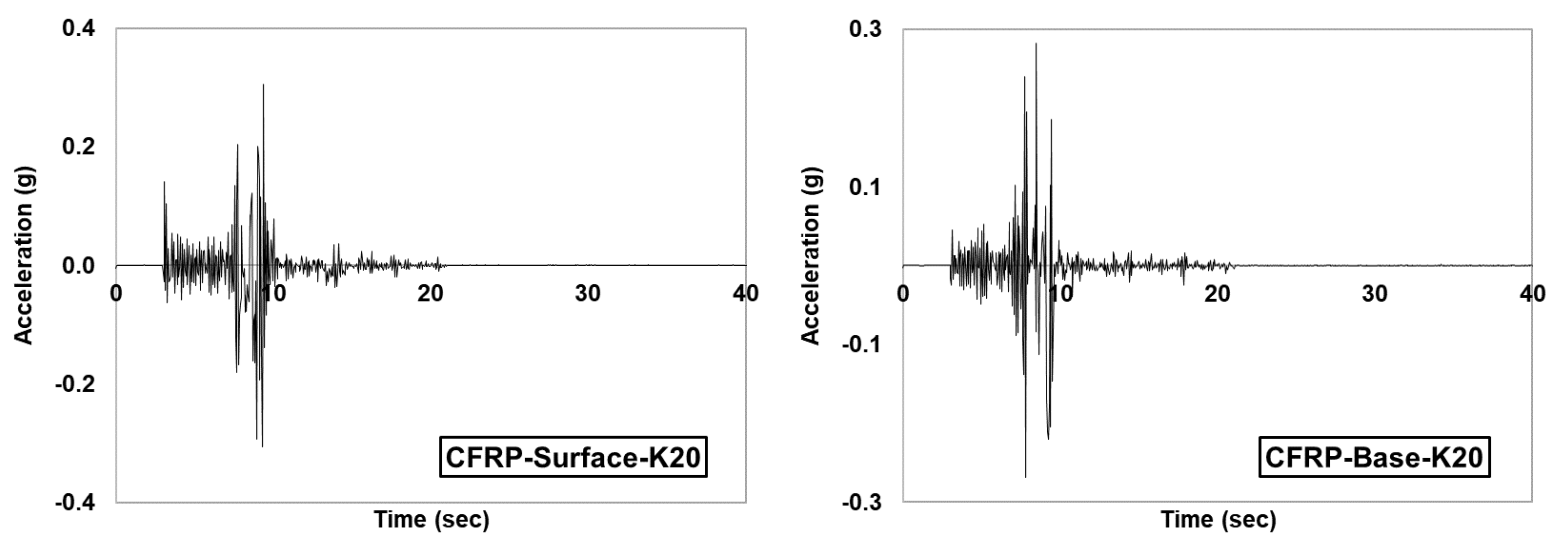

Figure 5.2: Sample acceleration time histories for the CFRP pile and free field response at base \& surface during the Kobe 20 excitation

The distribution of acceleration varies along the shaft of all model piles. Generally, FRP piles experienced higher accelerations close to their base (compared to the free field motion), followed by a rise in peak acceleration near the surface in all shaking events. The peak acceleration recorded on model FRP piles near surface (i.e., A5-CFRP and GFRP) were relatively close in terms of both amplitude and frequency content, however, aluminium piles showed significant amplification along their shaft (i.e., A6) and, consequently, considerable higher acceleration was recorded at the pile cap of aluminium (A8) compared to the pile caps motions of CFRP and GFRP. This means that superstructures rested on aluminium group piles would have experienced significantly greater acceleration compared to structures resting over FRP piles. This outcome can be correlated to the higher stiffness of conventional piles (i.e., aluminium) and hence higher deviation between the foundation and free field motions resulted from kinematic soil-pile interaction. The higher peak accelerations in the aluminium pile cap may also cause generation of the base shear and induce moments that consequently can appear as displacement, rocking motion and rotation of the foundation relative to ground surface. 
Table 5.1: Summarized peak accelerations at free field, pile cap and along the pile shafts for all events

\begin{tabular}{|c|c|c|c|c|c|c|c|c|c|c|}
\hline $\begin{array}{c}\text { Model } \\
\text { Piles }\end{array}$ & $\begin{array}{l}\text { Input } \\
\text { events }\end{array}$ & $\begin{array}{l}\text { Input } \\
\text { motions } \\
\text { (g) }\end{array}$ & $\begin{array}{l}\text { A1 } \\
\text { (g) }\end{array}$ & $\begin{array}{l}\text { A2 } \\
\text { (g) }\end{array}$ & $\begin{array}{l}\text { A3 } \\
\text { (g) }\end{array}$ & $\begin{array}{l}\text { A4 } \\
\text { (g) }\end{array}$ & $\begin{array}{l}\text { A5 } \\
\text { (g) }\end{array}$ & $\begin{array}{l}\text { A6 } \\
\text { (g) }\end{array}$ & $\begin{array}{l}\text { A7 } \\
\text { (g) }\end{array}$ & $\begin{array}{l}\text { A8 } \\
\text { (g) }\end{array}$ \\
\hline \multirow{6}{*}{$\begin{array}{c}\text { CFRP } \\
\text { and } \\
\text { AL } \\
\text { (T3) }\end{array}$} & 050 & 0.020 & 0.014 & 0.021 & 0.024 & 0.014 & 0.016 & 0.016 & 0.010 & 0.013 \\
\hline & 0100 & 0.041 & 0.032 & 0.041 & 0.041 & 0.031 & 0.031 & 0.049 & 0.049 & 0.059 \\
\hline & $\mathbf{O 2 0 0}$ & 0.082 & 0.057 & 0.066 & 0.059 & 0.050 & 0.051 & 0.052 & 0.088 & 0.098 \\
\hline & K5 & 0.041 & 0.041 & 0.080 & 0.105 & 0.052 & 0.058 & 0.060 & 0.210 & 0.252 \\
\hline & K10 & 0.082 & 0.088 & 0.204 & 0.186 & 0.147 & 0.110 & 0.159 & 0.490 & 0.595 \\
\hline & K20 & 0.164 & 0.172 & 0.306 & 0.283 & 0.187 & 0.210 & 0.295 & 0.812 & 1.05 \\
\hline \multirow{6}{*}{$\begin{array}{c}\text { GFRP } \\
\text { and } \\
\text { AL } \\
\text { (T4) }\end{array}$} & 050 & 0.020 & 0.014 & 0.025 & 0.021 & 0.041 & 0.015 & 0.019 & 0.014 & 0.018 \\
\hline & 0100 & 0.041 & 0.034 & 0.049 & 0.042 & 0.030 & 0.030 & 0.050 & 0.048 & 0.060 \\
\hline & $\mathbf{O 2 0 0}$ & 0.082 & 0.064 & 0.067 & 0.060 & 0.060 & 0.059 & 0.049 & 0.076 & 0.101 \\
\hline & K5 & 0.041 & 0.048 & 0.098 & 0.101 & 0.049 & 0.056 & 0.058 & 0.205 & 0.249 \\
\hline & K10 & 0.082 & 0.086 & 0.207 & 0.190 & 0.132 & 0.135 & 0.161 & 0.480 & 0.580 \\
\hline & K20 & 0.164 & 0.185 & 0.244 & 0.278 & 0.186 & 0.236 & 0.287 & 0.720 & 0.920 \\
\hline
\end{tabular}

\subsection{Spectral Analysis}

\subsubsection{Seismic Site Response: Free Field Motion}

Spectral analysis was used to examine the seismic response of different pile systems and the frequency content of earthquake motions measured on model piles and within the soil. Figure 5.3 demonstrates free field spectral response of the soil with reference to the base input motions during the $\mathrm{O} 100$ and $\mathrm{K} 20$ events. The free field motions within the soil were found to be similar in different depths for the $\mathrm{O} 100$ (similarly O50, K5) events, however, free field motion for strong motions such as K20 revealed rather comparably higher level of acceleration within the frequency range of 10-20 Hz, followed by a de-amplification as a result of higher levels of nonlinearity and possibly liquefaction occurrence of the soil. 

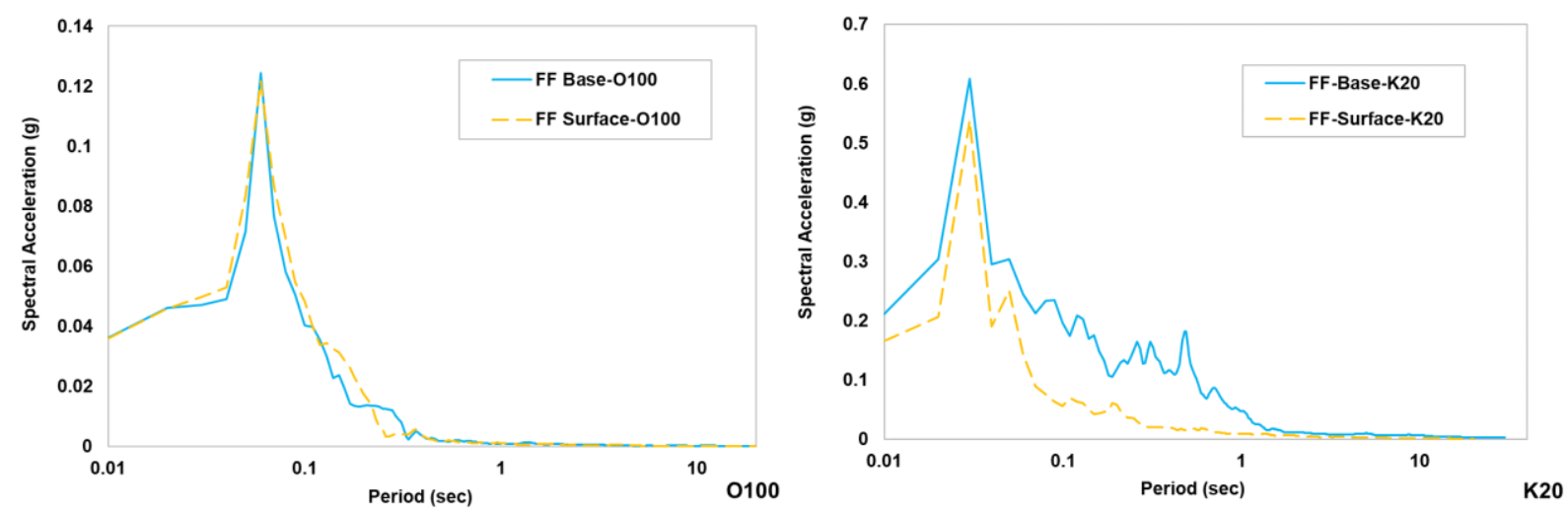

Figure 5.3: Response spectra for free field motions during the $\mathrm{O} 100$ and $\mathrm{K} 20$ shaking events

\subsubsection{Kinematic Soil-Pile Interaction (SPI)}

Previous studies have shown that the kinematic soil-structure interaction could deviate the foundation input motions from the free field motions and, hence, affect seismic response of superstructures through rocking motion due to base-slab averaging, large settlement and embedment effects (Stewart et al., 1999). This interaction can be defined as frequency dependant transfer function involving both the free field motion and the base-slab motion while the base and structure are assumed massless. To examine the influence of kinematic soil-pile interaction on foundation input motions, a pile/soil response spectral ratio (RRS) was defined to investigate this impact of kinematic interaction on pile foundation motions. To further examine the spectral ratio, two excitation events of $\mathrm{O} 100$ and $\mathrm{K} 20$ were selected to cover both weak and strong motions, respectively for the purpose of evaluating the interaction of the model piles (i.e., CFRP, GFRP, and AL). Figure 5.4 demonstrates the trends at two depths close to base and beneath the surface. 

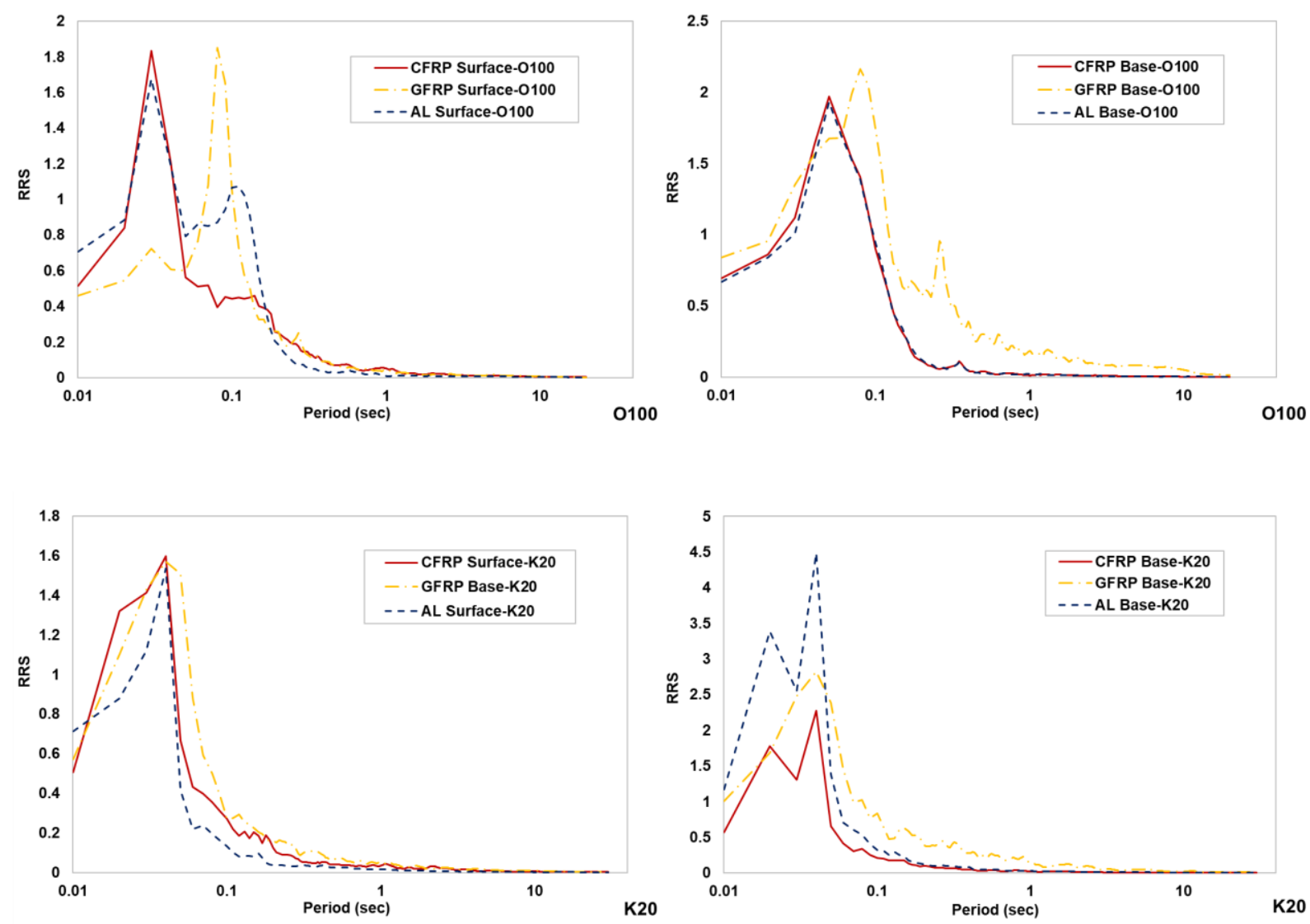

Figure 5.4: Ratio of response spectra (RRS) for all pile models during O100 and K20 shaking events

At $\mathrm{O} 100$, the overall spectral acceleration recorded on all model piles were shown to be approximately within 5-10\% range of those recorded at the same level within the soil (free field motion) and the ratio of foundation to free field motions were at about frequency range of 5-30 Hz. The recorded response ratio shows rather similar amplification along the pile for all model piles as expected. Although, the period of vibration for model piles varies as the aluminium pile showed longer period compared to FRP piles. This increase of spectral acceleration among model piles and change in frequency content of the seismic waves are evidently can be associated with the material characteristic of the pile materials compared to that of the soil and, hence, kinematic interaction between the model piles and soil. 
At the strong motion of K20, RRS values of the aluminium frictional piles shows severely higher amplification at base compared to both FRP model piles at frequency range of 10-30 Hz, which could be an indication of variation in the soil-pile interaction between FRP and conventional materials. Aside from the response at base of the pile, the RRS values at the surface show similar amplification and frequency content among all three model piles. Consequently, the response variation along the piles reveals slight variation between the frequency content (i.e., K20) of the accelerations recorded at base of the model piles and those recorded beneath the surface. These results indicate that the high-frequency portion of the input motion was significantly filtered due to the dynamic interaction of the surrounding liquefied soil around the piles. Interestingly, this filtering is not as significant for the acceleration recorded near the surface.

Additionally, considering the motion response for the two FRP materials (i.e., CFRP and GFRP), the GFRP frictional piles experienced slightly higher levels of acceleration at the base which led to higher amplifications compared to the CFRP piles. Therefore, it seems that the response of GFRP piles could be an indication of more dominant kinematic interaction compared to the CFRP ones especially during the frequency of about $20 \mathrm{~Hz}$. This behaviour has eventually led to larger levels of amplification for the GFRP piles compared to the CFRP pile group. The difference in seismic response of these two pile groups could be related to ductility of FRP material which is further investigated using findings of bending moments. Ultimately, the frequency content of the amplified motions appears to be consistent for all model pile groups. However, the kinematic soil-pile interaction seems to have slightly elongated the period of vibration for the GFRP piles from about $0.05 \mathrm{sec}$ to $0.1 \mathrm{sec}$.

To further assess the influence of pile materials on seismic response of superstructures, the spectral response of the pile caps for all model piles are illustrated in Figure 5.5. Predictably, all 
three piles did not respond strongly at lower excitation of O100, but the pile caps for all three model piles have experienced significantly larger accelerations resulted from significant kinematic SPI and possible rocking motion. The aluminium pile cap shows stronger rocking response compared to the FRP piles and this can indicate stronger superstructure accelerations. This could be attributed to higher rigidity of aluminium piles compared to the FRP piles $\left(\mathrm{E}_{\mathrm{AL}}=7.8 \mathrm{GPa}\right.$; $\left.\mathrm{E}_{\mathrm{CFRP}}=7.0 \mathrm{GPa} ; \mathrm{E}_{\mathrm{GFRP}}=5.8 \mathrm{GPa}\right)$. The peak accelerations measured on the pile caps exceeded the corresponding response quantities along the shaft of the model piles. The amplitude of spectral accelerations for FRP pile caps were somewhat similar, but the frequency content was marginally different. The predominant frequency of CFRP pile cap was recorded at $20 \mathrm{~Hz}$, while this was about $40 \mathrm{~Hz}$ for the GFRP pile cap. The pile cap spectra appear to be impacted by both the freefield and superstructure motions for the respective groups, signifying wave scattering and response delay.

Among the FRP piles, the pile cap for CFRP piles showed higher amplification than GFRP piles which may correspond to higher flexibility of GFRP piles compared to the CFRP piles. Therefore, the GFRP piles could be a suitable composite FRP option for pile foundations in seismic prone area for loose and liquefiable soils due to their higher flexibility and hence lower amplification of foundation input motions. 

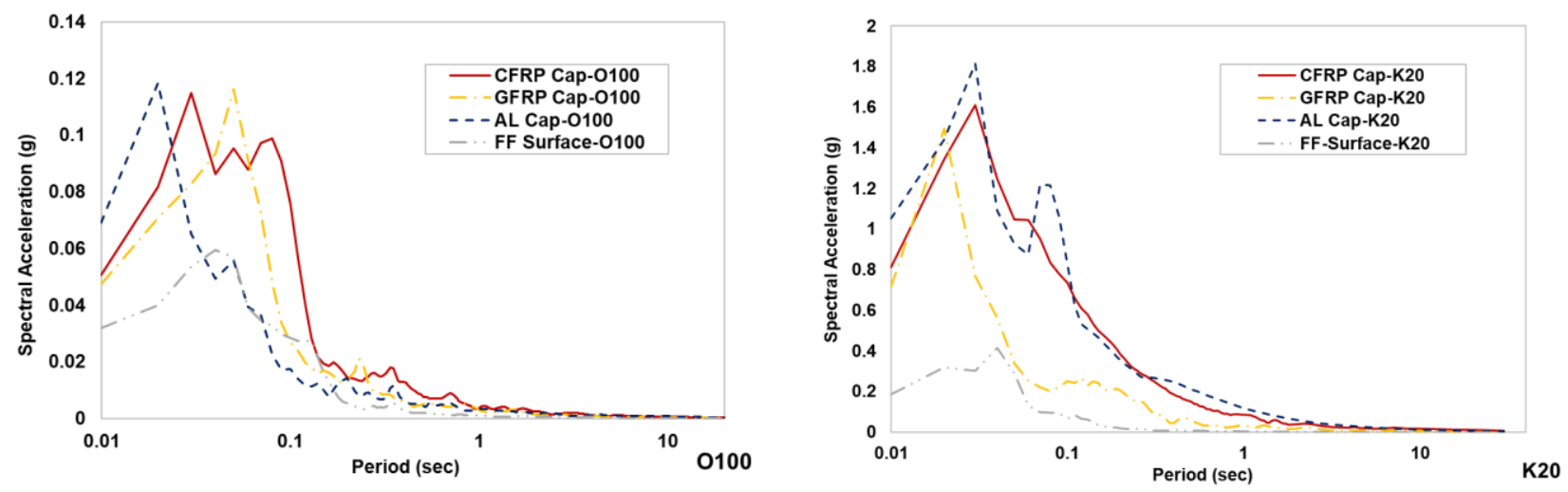

Figure 5.5: Pile cap reponse spectra at $\mathrm{O} 100$ and K20 shaking events for all model piles

\subsection{Pile Bending Moment}

Althoug, it may be sound to state that GFRP can be suitable alternative due its flexibility, however the result of bending moment at different excitations can clarify this statement as an additional indicator to underastand the bending response of the pile. Hence, a series of strain gauges were mounted over model piles at different depths to record possible lateral strains of the piles during shakings and, in turn, estimate bending moment envelope for the model piles. The bending moment envelope is defined by the absolute peak strain at each gauge during the excitation. This means that the result is not equivalent to actual bending moment diagram at the time step when the peak strain is recorded. As stated by the theory of elasticity and Hooke's law (Timoshenko, 1940), the generated moment in the pile section is a function of the recorded strain in the strain gauges, referring to the following equation:

$$
M=\frac{2 E_{p} I_{p}}{D} \times \varepsilon
$$

where, $E_{p}$ is the modulus of elasticity, $I_{p}$ is defined as the cross-sectional moment of inertia, $D$ is the outer diameter of the pile, and $\varepsilon$ is defined as the peak recorded strain in the strain gauges. 
Figure 5.6 illustrates measured response quantities and the change in the bending moment envelopes along the pile shaft for both the FRP pile groups and traditional pile (aluminium) at shaking event of O200, K20 (i.e., other excitations shown nearly identical responses). At O200 excitation with base acceleration of $0.082 \mathrm{~g}$, the distribution of the moment amplitude along the FRP piles and aluminium pile shafts reveal similar trends with slightly higher moment at aluminium mid-base. At K20 with stronger motion with peak input acceleration of $0.16 \mathrm{~g}$, higher shaking level resulted in higher amplitude of bending moment at the surface of CFRP and aluminium piles whereas the moment development along GFRP pile was subjected to lower bending moment near the pile head and experienced significant bending moment at its base. This type of behaviour seems to be as the result of glass fibre ductility defined as the ability of material to undergo large deformations/elongation but eventually may lead to failure of GFRP due to brittle characteristics along the shaft if stronger shaking applied.

Furthermore, aluminium piles developed slightly greater bending moments than CFRP piles along their shaft. This means aluminium piles may have experienced larger kinematic forces. On the other hand, this rise in bending moments, especially near the surface, suggests that liquefaction-induced kinematic loads dominated the pile response (Meymand, 1998). Although, this outcome may point out the material characteristic as controlling parameter on response of piles, however, surrounding soil response, and its deviation from spectral response of piles due to interaction and initiation of liquefaction, can be another valuable parameter to further explain the evident flexibility of CFRP compare to aluminium piles. 

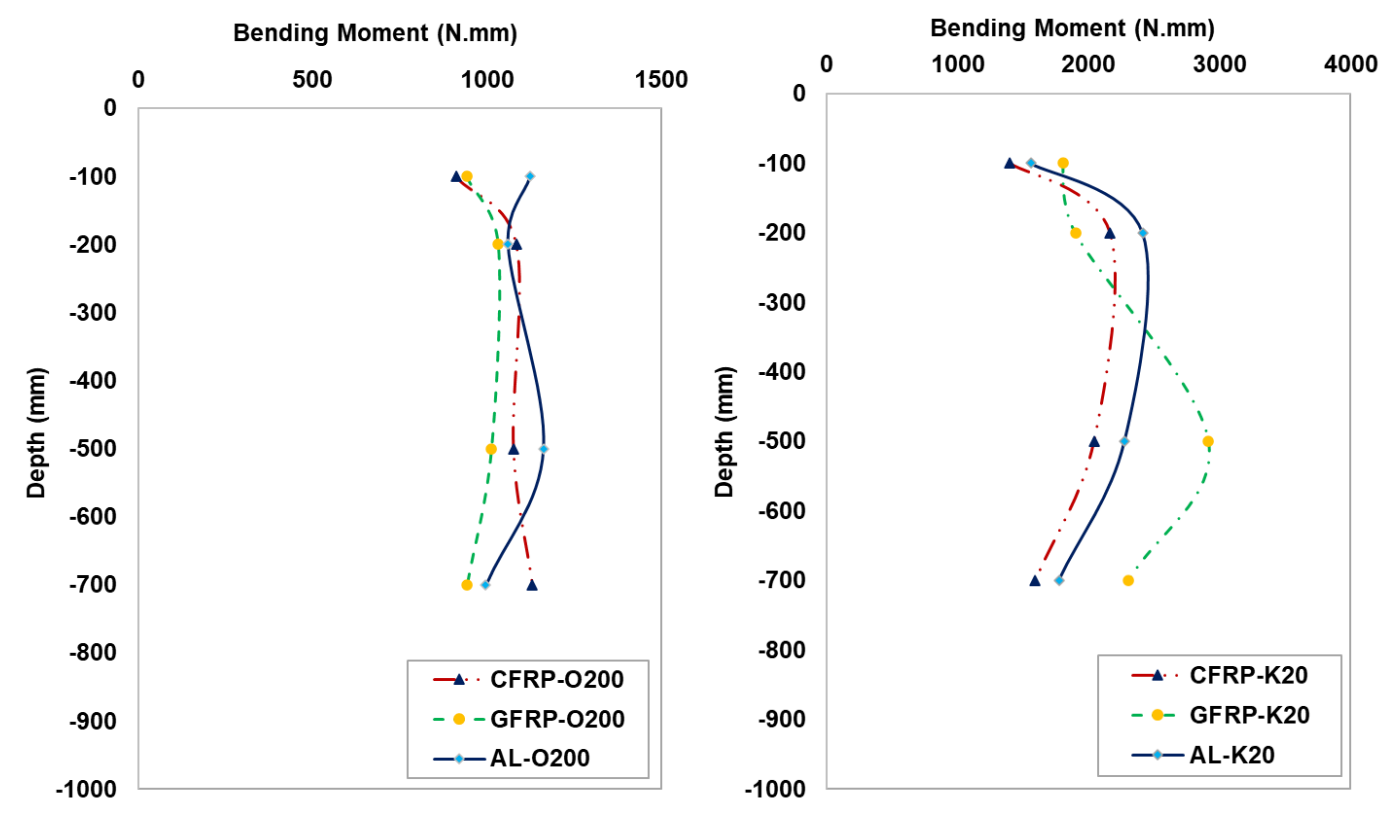

Figure 5.6: Recorded bending moment distribution along the piles at O200, and K20

Correspondingly, the generation of inertial forces from the cap and its superstructure masses may have induced and played additional role in developing the result of bending moments. To further validate these impacts, Figure 5.7 shows lateral deflections of the pile caps for all model piles during the K20 shaking. The amplitude of lateral displacement for CFRP and aluminium caps indicate similar trend which this can explain the similar bending moments at K20. These results also confirm that aluminium pile with high stiffness have exhibited less deformation.

Respectively, determination of lateral soil pressure of liquefied soil on piles during lateral spreading can be a matter of attention to many researchers since it is also essential to understand the role of lateral pressure exerted on these piles. Therefore, Figure 5.8 illustrates the lateral displacement of the laminar rings during K20 event near the surface and base of the laminar shear container. This further explains why model piles have experienced large bending moments close to the surface as the result of large lateral pressure employed on model piles at that level. 


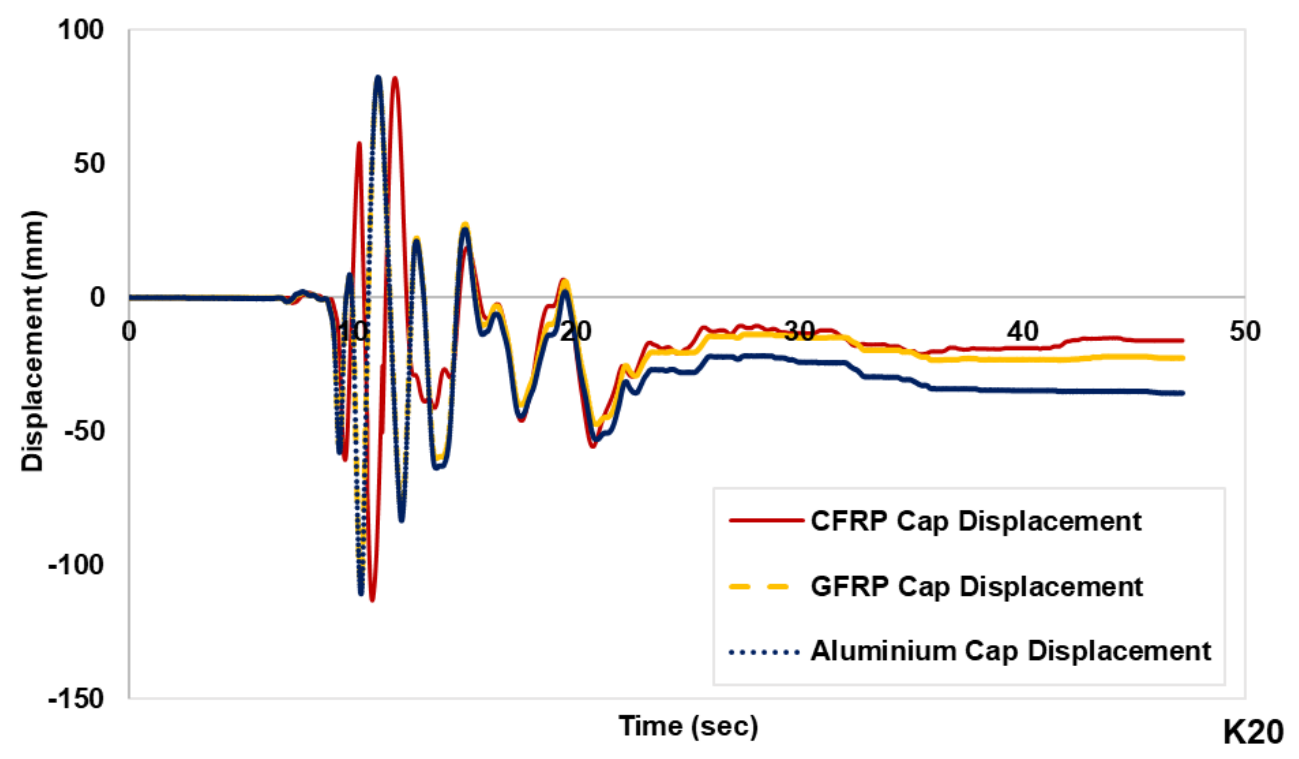

Figure 5.7: Pile head/cap deflection at the K20 event

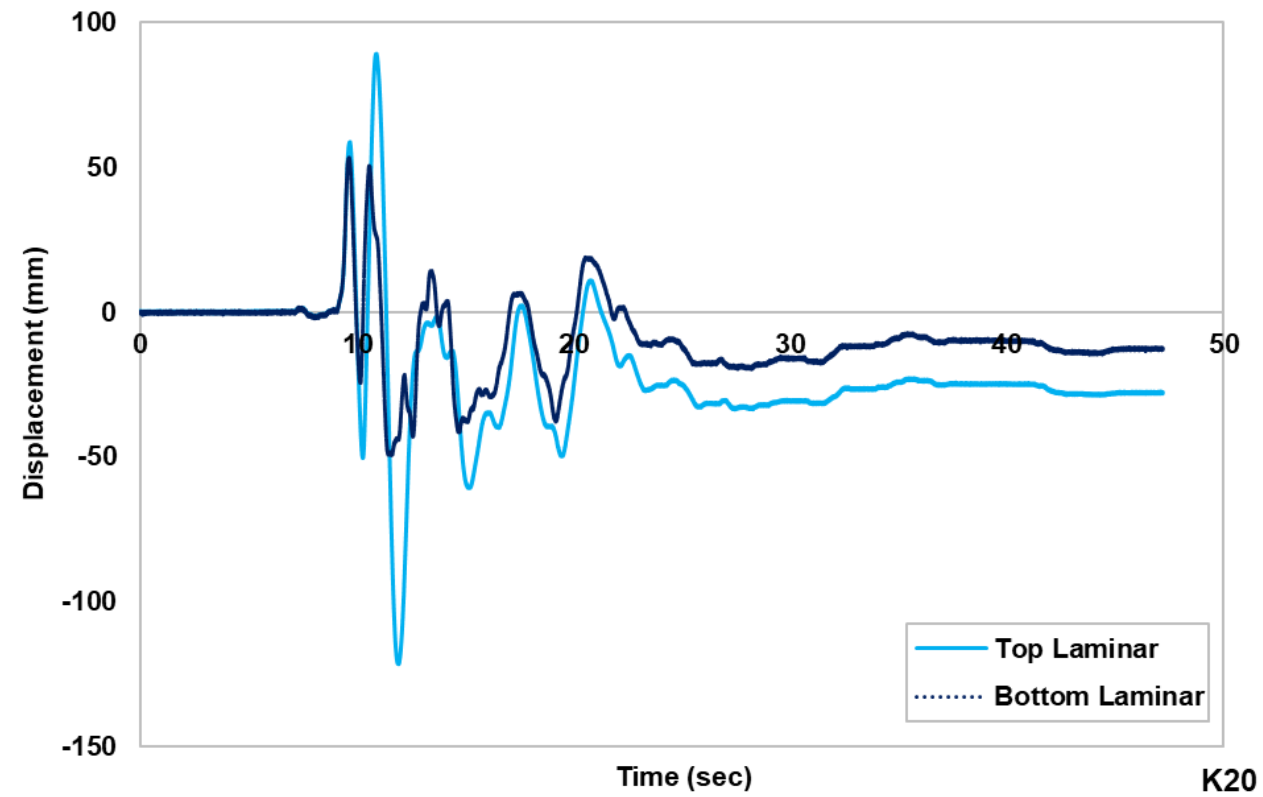

Figure 5.8: Laminar displacement at the K20 event 


\subsection{Pore Water Pressure and Liquefaction Assessment}

Past earthquakes (e.g., The Alaska, 1964 and Niigata earthquakes, 1964) resulted in disastrous failures of foundations and superstructures due to soil liquefaction (Seed et al., 1976). Shaking table tests can be adopted to understand generation and dissipation of pore water pressure (PWP) and, hence, the seismic behaviour of liquifiable soils and piles interaction. As stated, during the shaking events, excess pore water pressure was monitored using pore water pressure sensors at two designated depths of near the surface and base of the laminar container. A waiting time of 1520 minutes were typically adopted between each shaking event to assure full dissipation of PWP by monitoring data for PWP sensors.

From the recorded data, liquefaction triggering was evaluated by the excess pore water pressure ratio $\left(R_{u}\right)$ which is defined as the ratio of the excess PWP $(\Delta u)$ and the initial effective stress $\left(\sigma_{o}{ }^{\prime}\right)$, and additionally the estimation of the average relative density calculated from the volume change inside laminar container (i.e., settlement). This $R_{u}$ method was adopted due to its simplicity to determine the liquefaction potential within the free field boundary. Liquefaction is generally occurred if $R_{u} \geq 1$. During the Ottawa excitations (Figure 5.9), $R_{u}$ values did not reach the limit of 1.0 as weaker earthquakes with lower acceleration amplitude were intended to monitor the soil and FRP piles response as PWP increased in soil. Upon increasing the level of excitations by applying the Kobe events, further increase in pore water pressure led to generation of liquefaction in the soil.

To further examine liquefaction during seismic excitations, the surface settlement of the soil was estimated using a vertical LVDT and additional manual measuring after each shaking event. Figure 5.10 illustrates the measured result of settlement and estimated relative density which was confirmed to be uniform across the soil surface. These settlements can provide an indication 
of the contractive behaviour of the particles of liquefiable soil and consequently the rise in the soil density (Ueng et al. 2006). The initial average relative density of the soil was measured at about $49 \%$ prior to shaking tests. After first three shaking events (O50, O100, O200), the sand surface settled by about $2 \mathrm{~mm}, 3 \mathrm{~mm}$, and $3.5 \mathrm{~mm}$, respectively; as a result, the average relative density of the soil reached to approximately 54\%. Upon completion of each excitation, the PWP ratio tended to decrease (dissipation of PWP) as a result the soil became denser and its liquefaction resistance would have increased. At fourth excitation (i.e., K5), the development of liquefaction can be evidently seen followed by quick increase in settlement.

According to the $\mathrm{R}_{\mathrm{u}}$ value and settlement results, the soil deposits reached full liquefaction at two excitation stages of $\mathrm{K} 10$ and $\mathrm{K} 20$ with stronger motions, as expected. This eventually caused significant settlement at about $10 \mathrm{~mm}$ and $35 \mathrm{~mm}$, respectively with an immediate densification of soil with increase in relative density up to $77 \%$. Figure 5.11 presents stages of liquefaction development and clear observation of liquefaction as large quantity of sand boils were developed at the surface and around the piles at the last strong earthquake motion (K20). The occurrence of liquefaction was initiated during moderate shakings while complete liquefaction occurred under strong shaking. Additionally, it can be noted that the $\mathrm{R}_{\mathrm{u}}$ trend at surface may not reach the value of 1.0 since PWP readings were affected by the surface boundary condition and surface water sloshing, however the $\mathrm{R}_{\mathrm{u}}$ trend follows similar slope with trend of the base results. Although, the larger $\mathrm{R}_{\mathrm{u}}$ values (i.e., $\mathrm{K} 10$ and $\mathrm{K} 20$ events) at the base may potentially indicate that sensor may have shifted or dropped downward due to gravity and soil settlement which this may partially affect the measurement for PWP values. However, the overall outcome as indication of liquefaction can further explain the increase in bending moments near the surface as the liquefaction-induced kinematic loads dominated the pile's response due to lose of lateral support. 


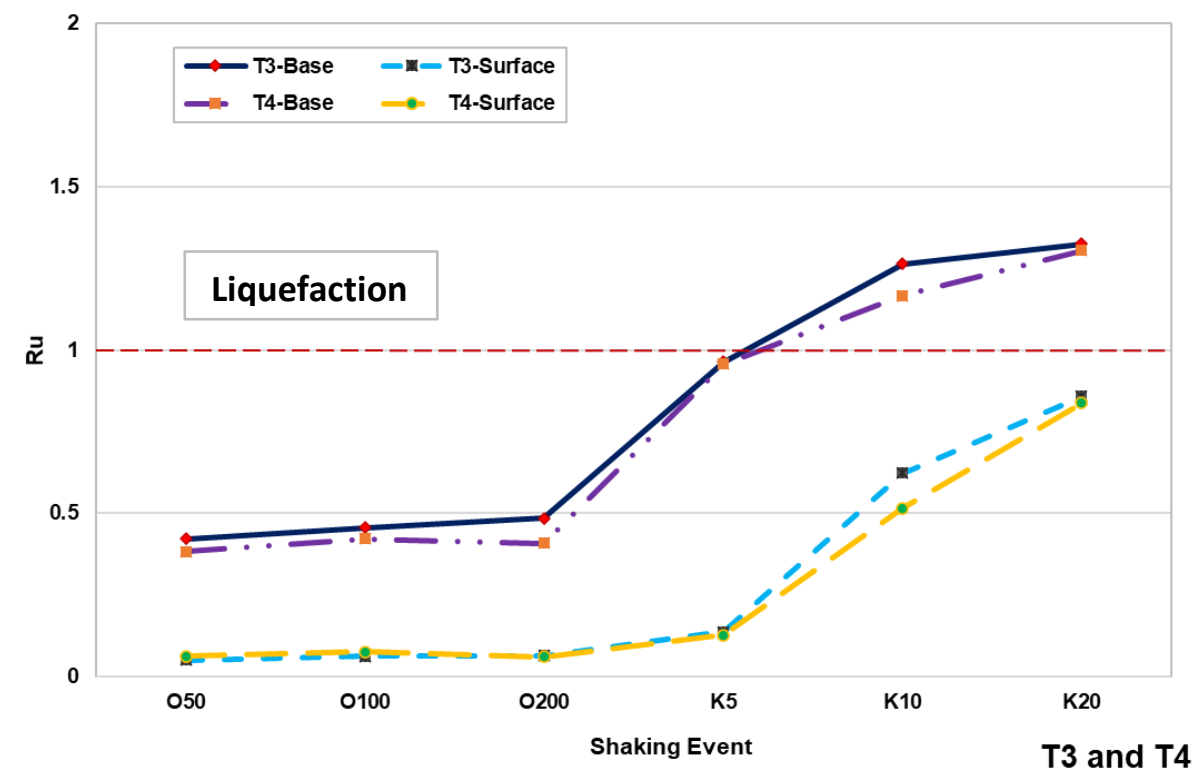

Figure 5.9: Rate of pore water pressure for all shaking events of T3 and T4

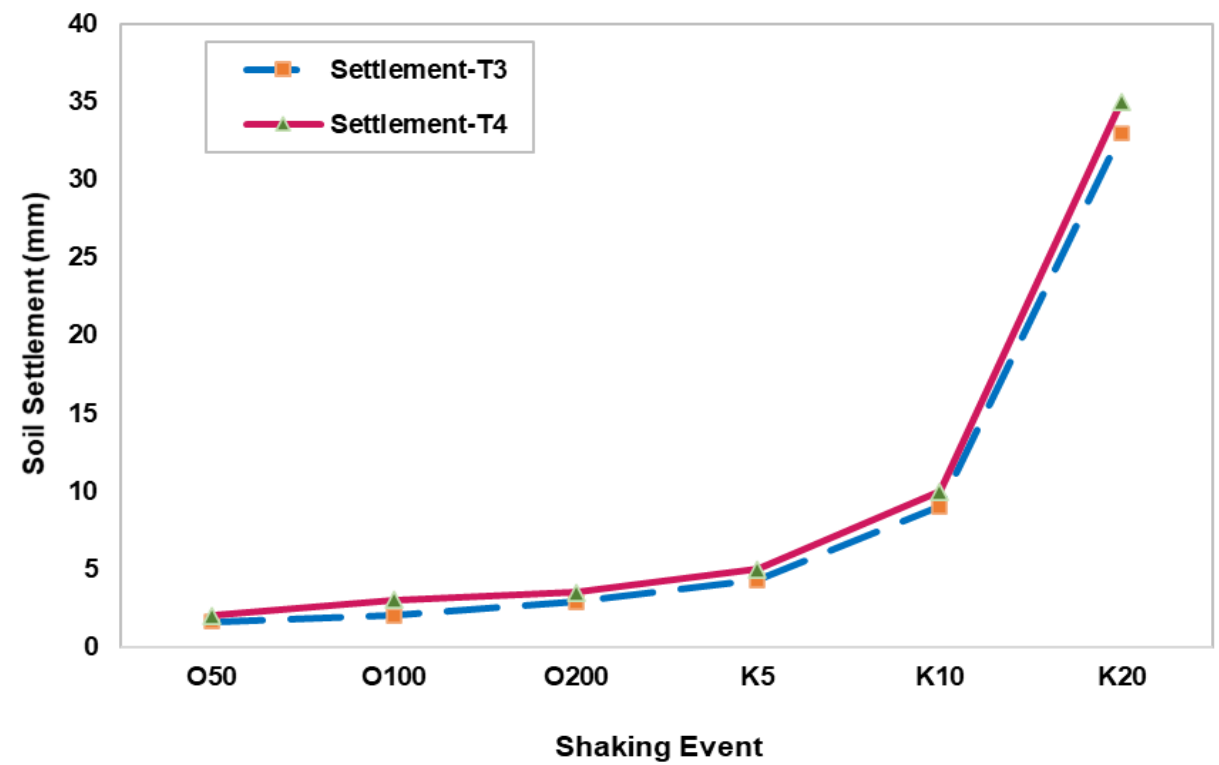

Figure 5.10: Settlement of soil deposit after each shaking event 


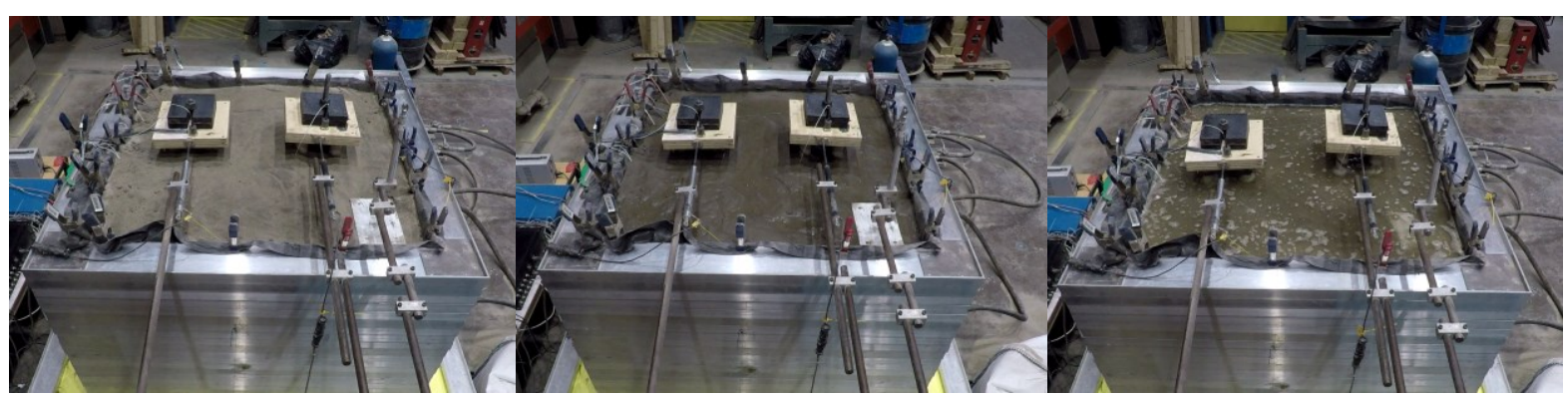

Figure 5.11: Development of sand boiling during the eaqrthquake loading (left to right)

\subsection{Summary}

In this study, two series of $1 \mathrm{~g}$ shaking table tests were conducted using laminar container as boundary on three frictional group pile models manufactured from Carbon Fiber-reinforced Polymer materials, Glass FRP, and conventional materials (i.e., aluminium) embedded in liquefiable sand. The soil-pile models were subjected to scaled input motions of 2010 Ottawa and the 1995 Kobe earthquakes. The following conclusions can be drawn from these shaking table tests:

1. The foundation motions recorded along the model piles showed significantly higher levels of excitation compared to the acclerations recorded within the soil at the same lavel. Most these amplifications were recorded at a period of $0.05-0.1$ seconds, corresponding to the frequency range of 10-30 Hz. This higher level of foundation motions recorded on the model piles were related to kinematic pile-soil interaction which deviated the foundation motions from that of the soil. The impact of such interaction was, however, different for each pile type. Foundation motions recorded on the aluminium piles as well as the pile cap were significantly higher than those recorded on FRP model piles. This was attributed to the higher rigidity and, hence, lower flexibility of conventional pile materials compared to the piles made of composite FRP materials. 
2. The elastic modulus (E) of the CFRP materials (as an indicator of stiffness and rigidity) was higher than the glass fibers at around 7.0 GPa compared to the GFRP materials at 5.8 GPa. Based on the results of the model piles, it can be stated that the higher rigidity of pile may proportionally cause to experience higher acceleration and hence higher-level of amplification of foundation input motions.

3. Pile materials may influence the seismic response of pile caps and its superstructure through kinematic soil-pile interaction and rocking motion. The aluminium pile cap experienced significantly higher acceleration and stronger rocking response compared to the FRP piles. Among the FRP piles, hollow GFRP pile was shown to be an appropriate alternative and replacement for frictional traditional piles. The GFRP pile can also be suggested as additional reinforcement for restoration and rehabilitation purposes of damaged traditional piles due to environmental factors.

4. The frequency content of the amplified motions appeared to be consistent for FRP model pile groups. Therefore, the soil-pile interaction has not deviated the frequency content of seismic motions recorded for both the conventional and FRP pile systems and only amplified the input motion.

5. Similarly, as the result of both shaking tests, increase of pore water pressure was observed, followed by the rearrangement of sand particles due to gravity. This tendency of contraction resulted in a sudden increase of pore water pressure which ended with liquefaction as demonstrated by determining ratio of pore water pressure. Ultimately, relative density of the soil increased at every shaking event which this can most likely increase the liquefaction resistance, as a consequence of sand densification. This can also 
explain the lower bending moment closer to base as the result of soil settlement (i.e., sand densification), hence this can cause larger kinematic forces near the surface. 


\section{Chapter 6: Seismic Response of End-Bearing Fibre-Reinforced Polymer (FRP) Piles in Soft Clay}

\subsection{Introduction}

The demand of using fibre-reinforced polymer (FRP) materials as a sustainable alternative in geotechnical applications has grown significantly in the last two decades. This surge of interest is due to advantages of FRP composite materials and recent advances in manufacturing ability of FRP materials in multiple forms including concrete filled FRP piles, hollow FRP tubes and FRP sheet piles (Guades et al., 2012). FRP can withstand aggressive corrosive environments such as the splash zone in case of marine piles, where they would be ultimately submerged and exposed (Stapleman, 1997). Similarly, highway overhead sign poles and pedestrian bridge piers need to retain their integrity in cold regions where salt is used for de-icing the roads. As an example, FRP round tubes are very light materials, which can enhance the specific strength and stiffness depending on fabric type. FRP tubes can be concentric in an axisymmetric pattern which is suited for applications involving loads applied from various directions such as wind pressure or it could be eccentric to provide more reinforcement in the tension. Therefore, there are demands of noncorrosive fiber-reinforced polymer composites as a potential alternative to increase service life, save on maintenance costs, and environmental benefits against harsh and corrosive environments.

Considerable research programs have been conducted to study the load transfer and flexural response of FRP piles to validate the application of FRP composites in construction industry (e.g., Iskander et al., 2001; Sakr et al., 2007; Hosseini and Rayhani, 2017). FRP materials have also recently been implemented in different forms in geotechnical industry. For example, FRP piles were used as a substitute to precast concrete piles for Route 40 highway bridge over 
Nottaway River in Virginia (Fam et al., 2003). In latest projects such as Statue Liberty Dock, more than 200 timber piles were replaced by FRP piles due to deterioration of the original piles. Long Beach bridge is another project where reinforced concrete piles were replaced with FRP pipe piles in New York. The lucrative capabilities of FRP composite piles encourages further investigation on performance of these piles under seismic loading in prone regions. Subsequently, a promising innovative form of FRP piling known as hollow FRP tube has recently been investigated under axial and lateral static loading (e.g., Giraldo and Rayhani, 2014) and there is lack of investigation to understand seismic response of these piles in soft clay deposits.

Past earthquakes have revealed complications due to the amplification of earthquake motion in soft clay deposits, and soil-pile-structure interaction (SPSI). Catastrophic structural damage and failure to pile foundations were observed in the Kobe (1995), Loma Prieta (1989) and Mexico City (1985) earthquakes, and clearly highlighted the role of local site conditions and soilstructure interaction (SSI) in modifying and altering the characteristics of strong motions (Nghiem, 2009; Mendoza and Romo, 1989). The challenge of soil-pile interaction in the seismic analysis and design of structures has become increasingly critical, as it may be inevitable to build structures at locations with less favourable geotechnical conditions in seismically active regions.

In this chapter, seismic performance of end bearing hollow FRP tubes made of carbon and glass fibers were studied using a series of shaking table tests. The impact of soil and piles' response on foundation input motion were analysed for both FRP tubes compared to conventional aluminium group piles in synthetic clay by considering SPSI, which is significantly important on the performance-based design of foundations in seismic regions. 


\subsection{Shaking Test Results}

\subsubsection{Acceleration Time Histories}

Figure 6.1 presents the locations of mounted instruments and designated subscription for each accelerometer (An)in the soil-pile model. A2 and A3 are accelerometers attached to a pile per group near the base; A5 and A6 were mounted on the same pile at a depth close to the surface; A1 and A4 are located inside the soil at the same level as A2 and A5 to record free field motions in the soil (far from the piles); A7 and A8 were mounted on the structure. Figure 6.2 shows sample acceleration times histories for the free field motion (A4) and corresponding pile motions for the CFRP (A5) and the conventional aluminium pile (A6) under the Kobe 20 shaking event. The peak accelerations in terms of ' $\mathrm{g}$ ' for each accelerometer for free field motions as well as both the pile groups are presented in Table 6.1.

From the time histories of acceleration, the soil conditions had a significant effect on the site response, as expected. The peak acceleration within the soil profile showed a major amplification from the base excitation to A1 and A4 along the soil profile for weaker seismic events (e.g., O50 and O100). As the input acceleration at the base of the soil model increased, the free filed motion at both the soil levels (A1 and A4) showed a decreasing trend (de-amplification). As it will be discussed later, this reduction in free field motion could be related to higher strain induced in the soil and the soil nonlinear response during stronger input motions. However, the recorded peak accelerations within the soil (free field) for all applied excitations were significantly less than those measured on piles at the same depth. This difference in peak ground motions underscores the importance of considering soil-pile interaction impacts on foundation input motions in seismic prone areas. 
The distribution of acceleration varies along the shaft of all model group piles. The FRP piles experienced smaller acceleration close to their base and followed by a rise in peak acceleration near the surface in all shaking events. This behaviour was similarly observed in response motion of Aluminium piles along the shaft. Although, there was significantly higher response at the aluminium pile cap compared to the CFRP/GFRP group pile caps. This also means that superstructures rested on aluminium group piles would have experienced significantly greater acceleration compared to structures resting over FRP piles. This can be related to the higher stiffness of the traditional piles (i.e., aluminium) and hence higher deviation between the foundation and free field motions resulted from kinematic soil-pile interaction. The higher peak accelerations in the aluminium pile cap may also result in generation of the base shear and induced moments that consequently can appear as displacement and perhaps rotation of the foundation relative to the free field.

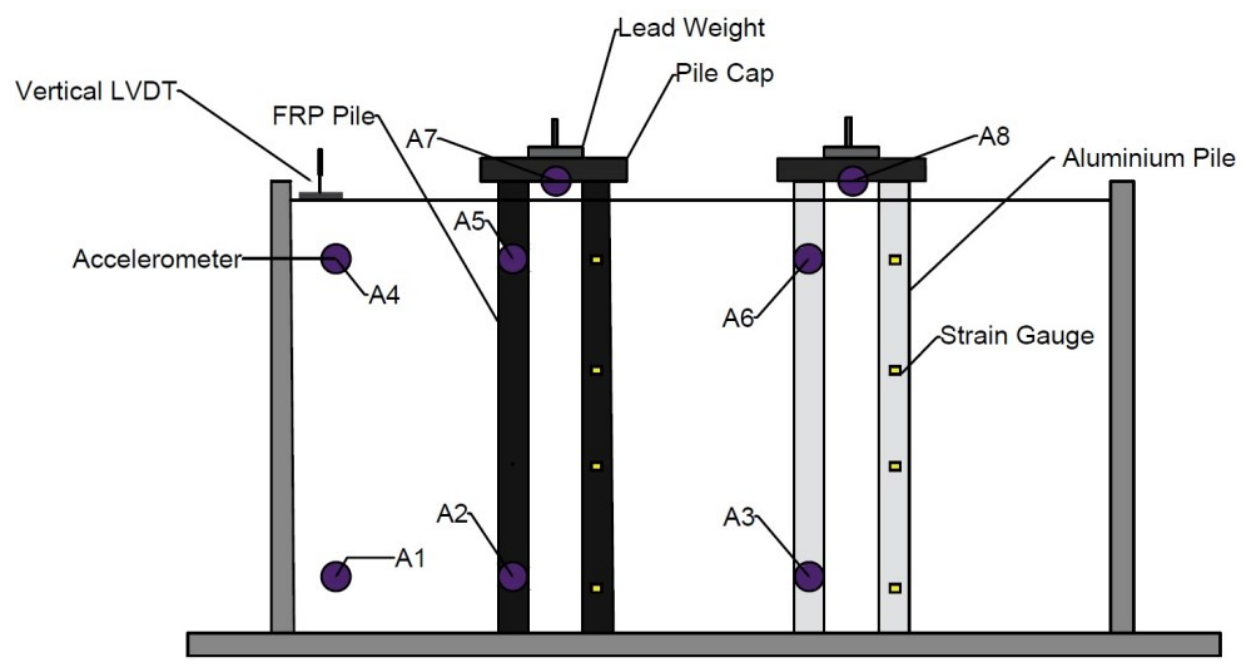

Figure 6.1: Shaking table models' configuration in laminar box. 

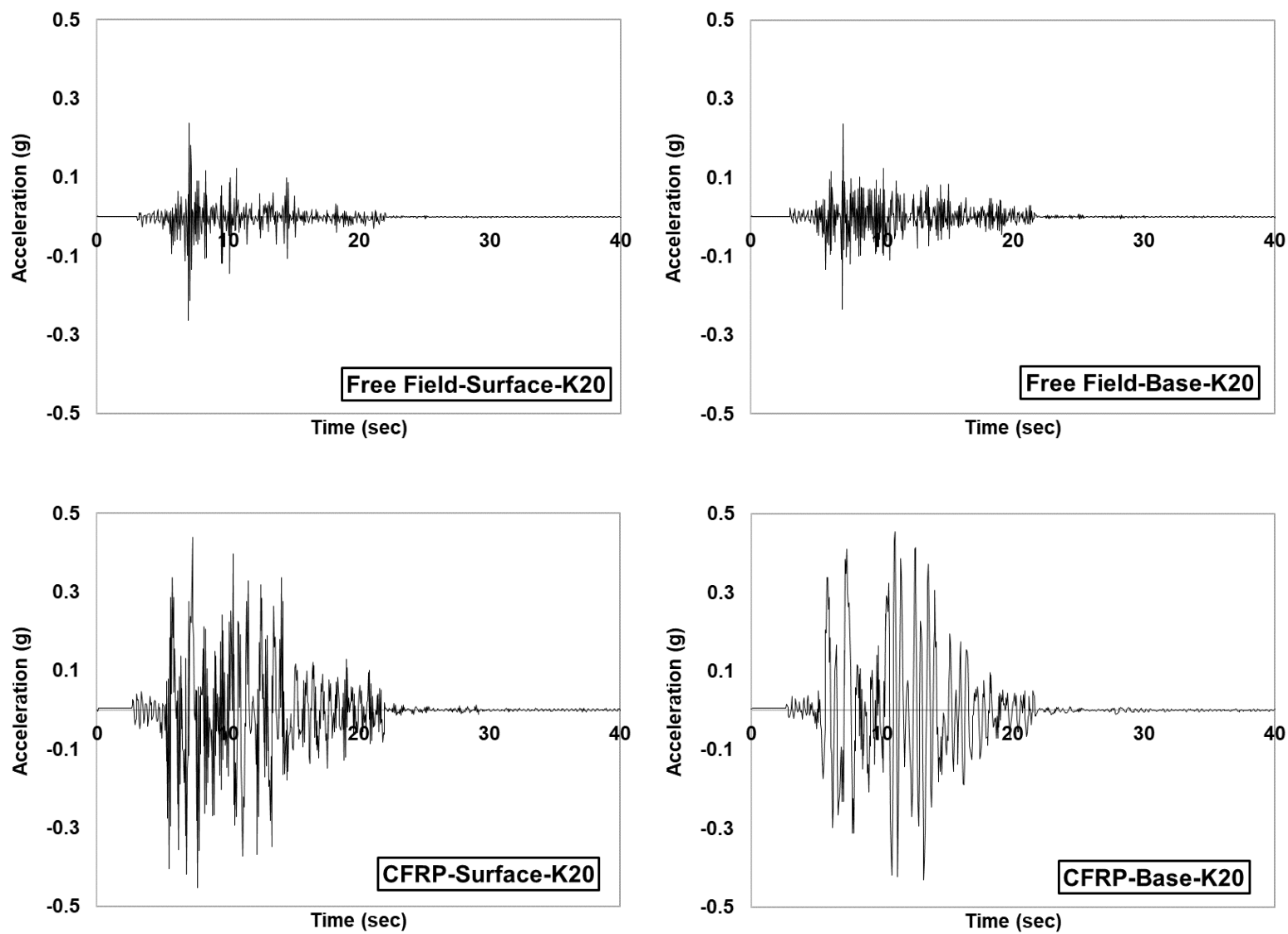

Figure 6.2: Acceleration time histories free field and CFRP at K20 shaking

Table 6.1: Summarized peak accelerations at free field, pile cap and along the pile shafts for all events

\begin{tabular}{|c|c|c|c|c|c|c|c|c|c|c|}
\hline $\begin{array}{c}\text { Model } \\
\text { Piles }\end{array}$ & $\begin{array}{l}\text { Input } \\
\text { events }\end{array}$ & $\begin{array}{c}\text { Input } \\
\text { motions } \\
\text { (g) }\end{array}$ & $\begin{array}{l}\text { A1 } \\
\text { (g) }\end{array}$ & $\begin{array}{l}\text { A2 } \\
\text { (g) }\end{array}$ & $\begin{array}{l}\text { A3 } \\
\text { (g) }\end{array}$ & $\begin{array}{l}\text { A4 } \\
\text { (g) }\end{array}$ & $\begin{array}{l}\text { A5 } \\
\text { (g) }\end{array}$ & $\begin{array}{l}\text { A6 } \\
\text { (g) }\end{array}$ & $\begin{array}{l}\text { A7 } \\
\text { (g) }\end{array}$ & $\begin{array}{l}\text { A8 } \\
\text { (g) }\end{array}$ \\
\hline \multirow{6}{*}{$\begin{array}{c}\text { CFRP } \\
\text { and } \\
\text { AL } \\
\text { (T1) }\end{array}$} & O50 & 0.020 & 0.032 & 0.061 & 0.076 & 0.024 & 0.059 & 0.081 & 0.045 & 0.098 \\
\hline & 0100 & 0.041 & 0.071 & 0.100 & 0.121 & 0.067 & 0.094 & 0.129 & 0.097 & 0.140 \\
\hline & $\mathbf{0 2 0 0}$ & 0.082 & 0.105 & 0.219 & 0.217 & 0.101 & 0.185 & 0.221 & 0.175 & 0.259 \\
\hline & K5 & 0.041 & 0.075 & 0.121 & 0.157 & 0.076 & 0.110 & 0.169 & 0.101 & 0.179 \\
\hline & K10 & 0.082 & 0.131 & 0.244 & 0.258 & 0.163 & 0.273 & 0.293 & 0.263 & 0.297 \\
\hline & K20 & 0.164 & 0.216 & 0.455 & 0.490 & 0.252 & 0.448 & 0.539 & 0.419 & 0.590 \\
\hline \multirow{6}{*}{$\begin{array}{c}\text { GFRP } \\
\text { and } \\
\text { AL } \\
\text { (T2) }\end{array}$} & 050 & 0.020 & 0.040 & 0.076 & 0.071 & 0.030 & 0.058 & 0.078 & 0.038 & 0.097 \\
\hline & 0100 & 0.041 & 0.081 & 0.124 & 0.116 & 0.055 & 0.117 & 0.127 & 0.108 & 0.147 \\
\hline & $\mathbf{O 2 0 0}$ & 0.082 & 0.104 & 0.197 & 0.224 & 0.108 & 0.181 & 0.235 & 0.176 & 0.264 \\
\hline & K5 & 0.041 & 0.080 & 0.119 & 0.152 & 0.085 & 0.105 & 0.162 & 0.104 & 0.178 \\
\hline & K10 & 0.082 & 0.138 & 0.201 & 0.271 & 0.160 & 0.272 & 0.291 & 0.273 & 0.303 \\
\hline & K20 & 0.164 & 0.239 & 0.514 & 0.510 & 0.259 & 0.498 & 0.557 & 0.534 & 0.575 \\
\hline
\end{tabular}




\subsection{Spectral Analysis}

\subsubsection{Free Field Soil Response}

It is now a well-established fact in geotechnical earthquake engineering that soft soil deposits can amplify the seismic bedrock motion through the soil column toward the surface. Soft clay deposits are recognised to specifically amplify shaking in weaker seismic events, though very strong shaking (e.g., K20) may attenuate surface motions due to soil non-linearity and stiffness degradation. It can also be hypothesized that such motions, however, may induce shear failure in soft soil deposits.

The free field response of the soil was established based on results from the two accelerometers positioned inside the laminar shear container far from pile foundations. From the collected acceleration data (Table 6.1), spectral response was established to describe the frequency content of the earthquake motions measured on the piles and within the soil (Figure 6.3). Free field acceleration response profile revealed significantly higher response acceleration within the soil profile compared to the input motion in relatively weaker events, which is signifying the amplification of motions along the soil profile. This behaviour can be observed in $\mathrm{O} 100$ and K5 free-field response where peak input motion of $0.041 \mathrm{~g}$ is clearly demonstrating amplification response of soil and elastic behaviour of soil at base and surface. As the intensity of input acceleration in the soil models increased (e.g., K20), the level of amplification in the soil profile decreased. Correspondingly, lower amplification for soft soils exposed to strong shakings was reported which followed by the nonlinear response of soft clay under strong excitations as well as stiffness degradation. Furthermore, the higher peak amplitude of the ground acceleration and strain levels in the soil is associated with higher material damping which further reduces the response as 
the soil may have experienced shear failure limiting the stress that can be transmitted to the surface soil layers.
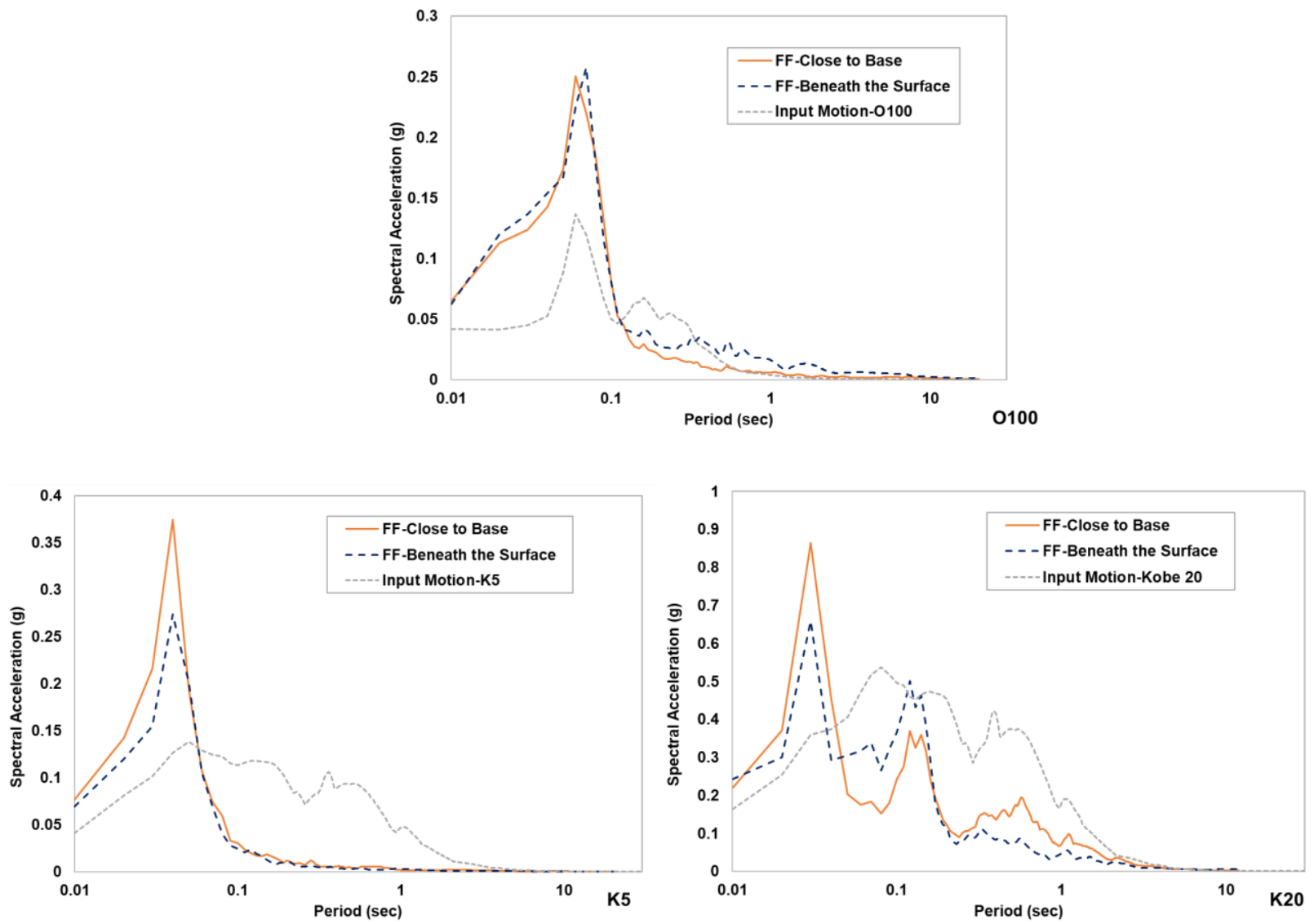

Figure 6.3: Response spectra for free field motions during the O100, K5 and K20 shaking events

\subsubsection{Pile- Soil Interaction Analysis}

Previous studies have shown that the kinematic soil-structure interaction could deviate the foundation input motions from the free field motions and, hence, affect seismic response of superstructures. This interaction can be defined as frequency dependant transfer function involving both the free field motion and the base-slab motion while the base and structure are assumed massless. As a result, this may cause foundation rocking due to base-slab averaging and embedment effects (Stewart et al., 1999). This impact can be explained by assessing the kinematic 
soil-pile interaction on foundation input motions. The spectral acceleration closer to ground surface for all the model piles were compared with the spectral response of the free field motions at the same depth. A pile/soil response spectral ratio (RRS) can evidently illustrate the divergence of the pile behaviour throughout the shaft as the result of its adhesion and material characteristics which affect the pile foundation motions. The results of spectral ratio are plotted for all the model piles measured at the O100, K5 and K20 events in the following Figure 6.4.

The spectral acceleration recorded on all the model piles were indicated to be higher than those recorded at the same level within the soil (free field motion) by almost 2 times. The ratio of foundation to free field motions were substantially higher at the frequency range of 1-20 Hz. The period of vibration was also shown to be higher for the foundation motions compared to the free field records. This increase in spectral acceleration for the pile models and change in frequency content of the seismic waves are clearly associated with the difference in flexural stiffness of the pile materials compared to that of the soil and, hence, significant kinematic collaboration between the model piles and soil. This result can be attributed to the inability of the foundation to follow the wave motion due to the flexural rigidity of the piles. Therefore, during cyclic or earthquake loading, seismic waves are reflected as the foundation is being stressed by seismic loading and the foundation will experience oscillatory motion that differs substantially from the free field response. 

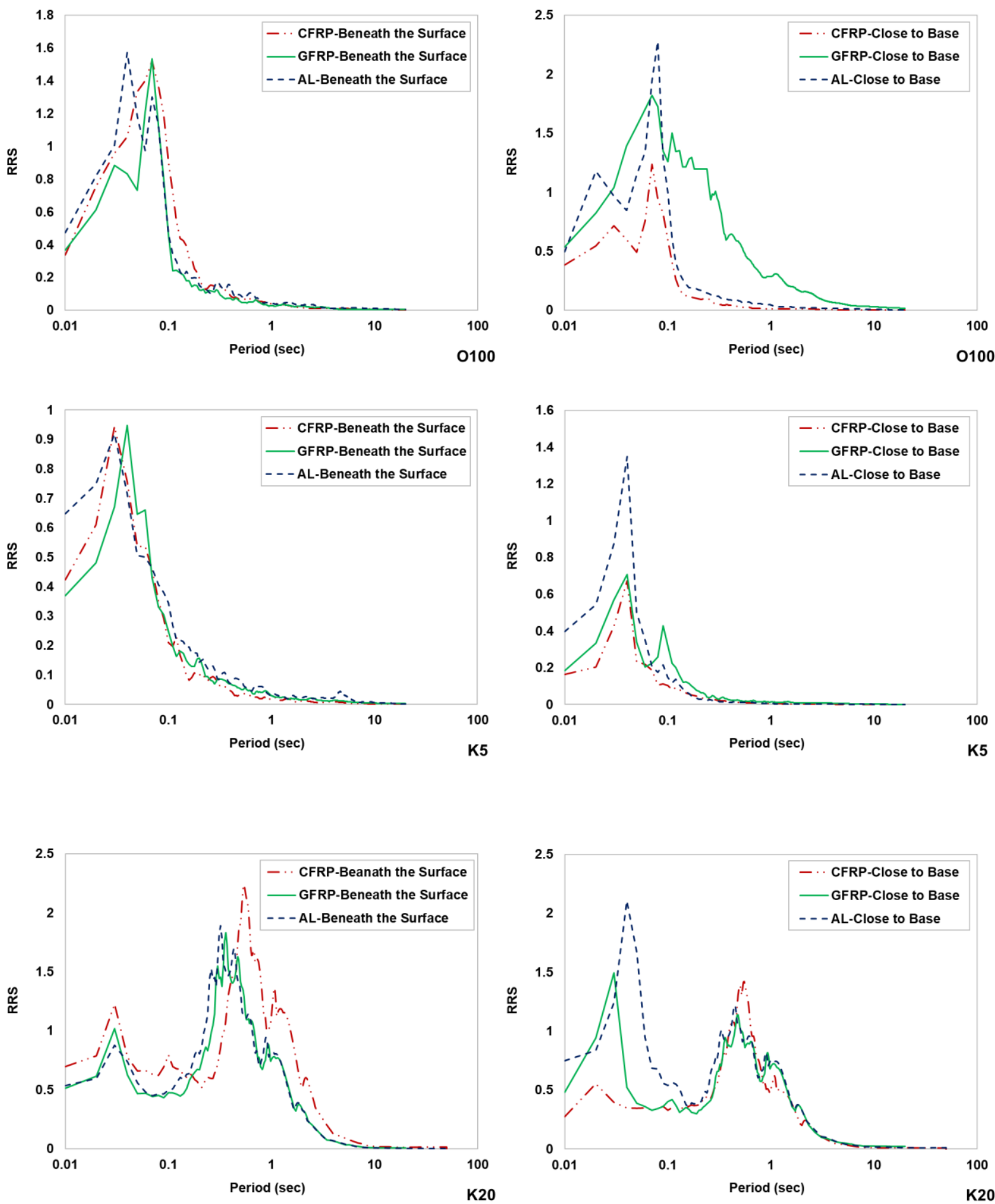

Figure 6.4: Pile/soil response spectral ratio (RRS) for all models during O100, K5 and K20 shaking events 
An equivalent comparison can be made for the different model piles to evaluate the impact of pile materials on the pile-soil interaction and, in turn, the seismic response of different foundation systems using the spectral acceleration response of piles. Figure 6.5 is evidently showing similar linear response of FRP piles and aluminium pile at $\mathrm{O} 100$ and $\mathrm{K} 5$ excitations near the surface, while larger amplification was felt at the base of aluminium pile as a result of the kinematic forces at the direction motion close to the base. Nevertheless, the frequency due to shaking was substantially filtered at the surface and alternatively continued at linear state. This outcome is correspondingly differed at stronger motion of K20, where higher RRS values were observed with drastically greater amplifications at the same frequency level of 10-100 $\mathrm{Hz}$, and 1$10 \mathrm{~Hz}$ at both locations (BS and $\mathrm{CB}$ ). This response is attributable to the applied ground motion that aluminium piles experienced with almost twice the free field response at its toe. This means the response of aluminium piles were dominated at the base by kinematic forces as reverse of same trend was observed near the surface where CFRP group piles felt slightly larger spectra ratio with respect to its free field response, at frequency range of 1.0-10 Hz. However, it is important to notice that free field conditions can certainly influence the performance of these model piles as their material characteristics (e.g., flexural stiffness) may also be another parameter to develop this particular behaviour. Therefore, the results may reveal the meticulous analysis of spectral response of each model pile at different shaking stage, as it was anticipated, the end-bearing aluminium group piles have suffered larger deformation and magnitude of acceleration at the toe. 

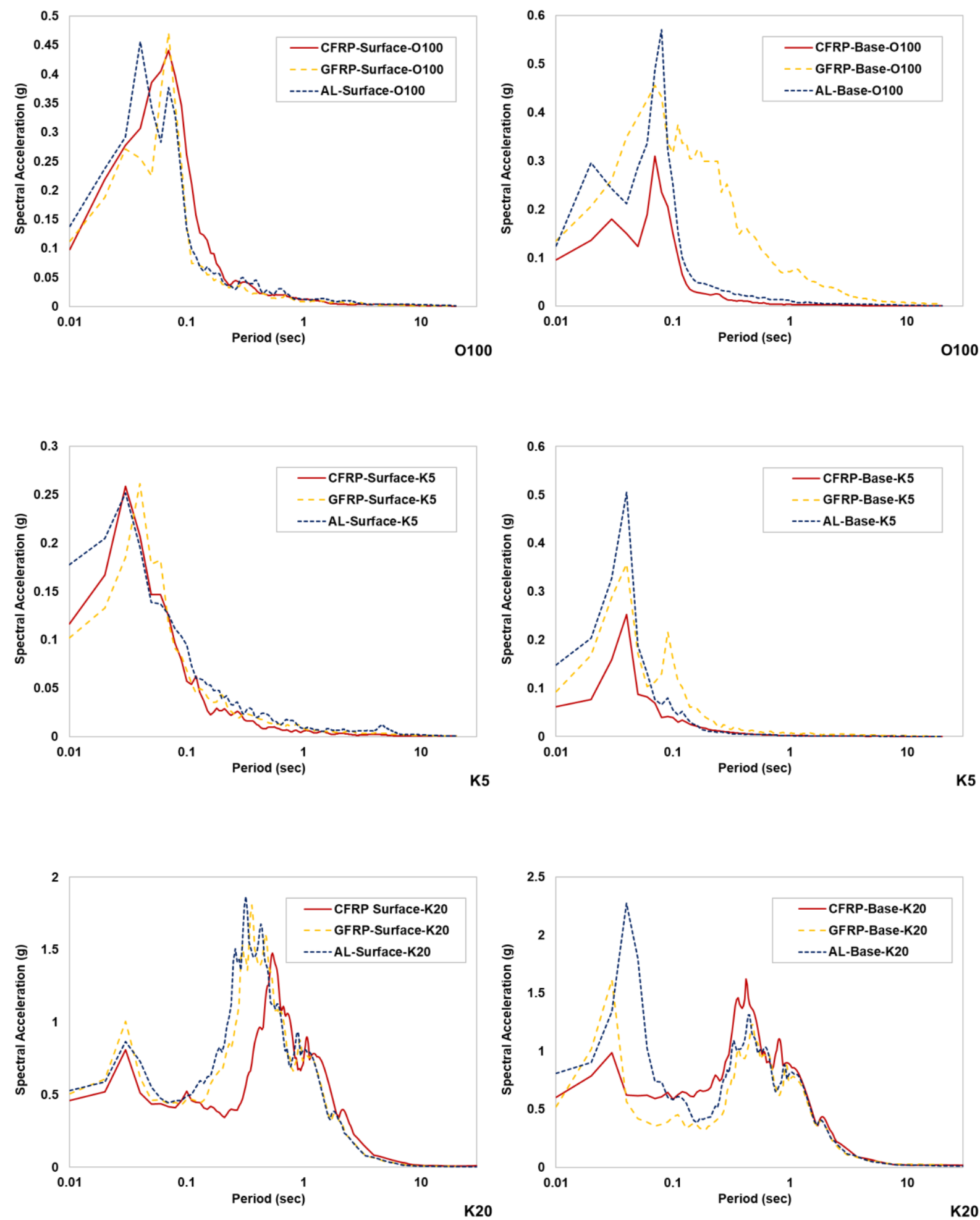

6.5: Spectral acceleration of model piles at O100, K5, and K20 excitations 
Alternatively, comparing response of both FRP piles at Kobe20 (i.e., CFRP and GFRP) shows that the CFRP piles experienced slightly higher levels of acceleration and led to higher amplifications compared to the GFRP piles near the surface. Therefore, it seems that the response of CFRP piles could be an indication of more dominant interaction than the GFRP. As stated earlier, the elastic modulus (E) of the CFRP materials (as an indicator of stiffness and rigidity) was higher than the glass fibers at around 7.0 GPa compared to the GFRP materials at 5.8 GPa. This difference in rigidity could have led to higher motions transferred through the CFRP piles and hence higher-level amplification of foundation input motions affected the overall response as also observed in traditional piles. Nevertheless, the frequency content of the amplified motions appears to be consistent for all model pile groups. Thus, the soil-pile interaction has not deviated the frequency content of seismic motions recorded for the pile systems and only amplified the input motion.

\subsubsection{Impact of SPI on Pile Cap Motion}

The characteristic of earthquake motions at the structure is affected by the response of the underlying soil through soil amplification and soil-structure interaction. This behaviour can further be examined by the influence of pile materials on seismic response of structures (or vice versa). The spectral response of the pile cap were compared for all model piles using the collected data from attached accelerometer on the superstructure of each pile group. As expected, the pile caps for both the FRP piles have experienced similar response which could correspond to close ductility of materials since higher amplification was measured on the aluminium pile cap. This reveals that the composite material characteristics including their ductility could have critical impact on the response of the pile along the shaft, pile cap, and the structure. 
Figure 6.6 demonstrates the response spectra measured on accelerometers placed over the pile caps under three seismic excitations (O100, K5, and K20). Both FRP pile caps demonstrated rather similar amplification, and it was much lower than what experienced on the aluminium pile cap and its structure. During the K5 event, the high-frequency portion of input motions was significantly filtered at surface of the piles which is due to the dynamic interaction of the surrounding soil with the piles. Interestingly, this filtering is not observed for the acceleration recorded at the aluminium pile cap, but the cap experienced almost $40 \%$ increase in acceleration from the response of input motion.
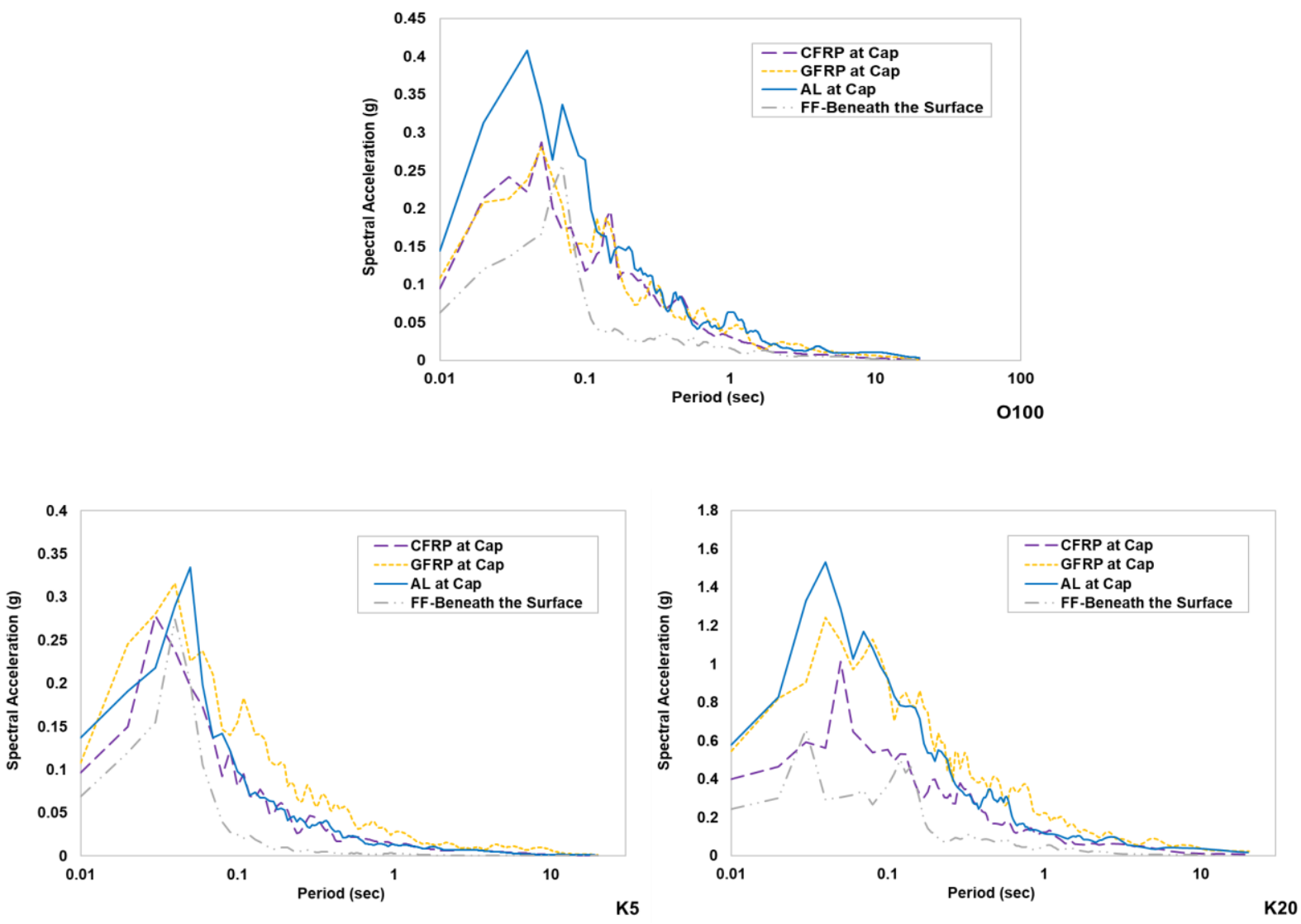

Figure 6.6: Pile cap reponse spectra at K5 and K20 shaking events for all model piles 
Greater flexibility of FRP piles and the difference in the interconnection of FRP piles to the cap may split the kinematic forces along the pile shaft through the cap and its structure, whereas traditional aluminium piles have reflected the induced kinematic forces to the cap and structure which this can result on damage or failure of structural members including partitions of any structure. Consequently, FRP composites may be a suitable option compared to traditional piles especially in seismic prone areas and offshore projects due to its larger flexibility and of lower intensification of foundation input motions.

\subsection{Effect of Shaking on Pile-Soil Interface}

During seismic loading, pile-soil gaps may develop between the soil and the pile near the ground surface which could result in a reduction of soil-pile interface strength. This condition may further affect the overall soil-pile-structure interaction as radiation damping may also occur due to the lower stiffness contrast between the soil and piles. Additionally, piles may be displaced in direction of motion at much higher frequencies than the surrounding soil, and soil-pile contact may force the soil to also oscillate at these high frequencies, resulting in the transmission of high frequency energy away from the pile into the surrounding soil (Meymand, 1998). On the other hand, since radiation damping occurs at high frequencies and low levels of soil damping, and it cannot propagate through the established gaps launched between the pile and soil, it can further affect the response of the pile cap due to the criticality source of radiation damping. Consequently, it dominates the structure's inertial forces and reduces the overall effects of the spectral deamplification.

It is essential to comprehend that upon completion of $\mathrm{K} 20$, model piles have experienced gapping (Figure 6.7). This shaking induced gapping could lead to some strength degradation 
contributed to a partial loss of soil-pile adhesion. This may have played a role in the level of acceleration recorded on piles (close to surface) and pile caps through reduction of radiation damping and contributing to amplification of foundation input motions. Although, all pile caps were loaded at constant light head mass, though at strong shaking of K20, a small partial inertial interaction could also have caused subsequent near surface soil-pile gapping and softened zones. The soil-pile cap was found to be slightly wider for the aluminium piles compared to the FRP model piles at K20 event. Therefore, it is probable that the response of aluminium pile cap was related to the gapping at the surface where potentially significant amplification was felt at the head/cap due to lose of soil-pile interface stiffness.
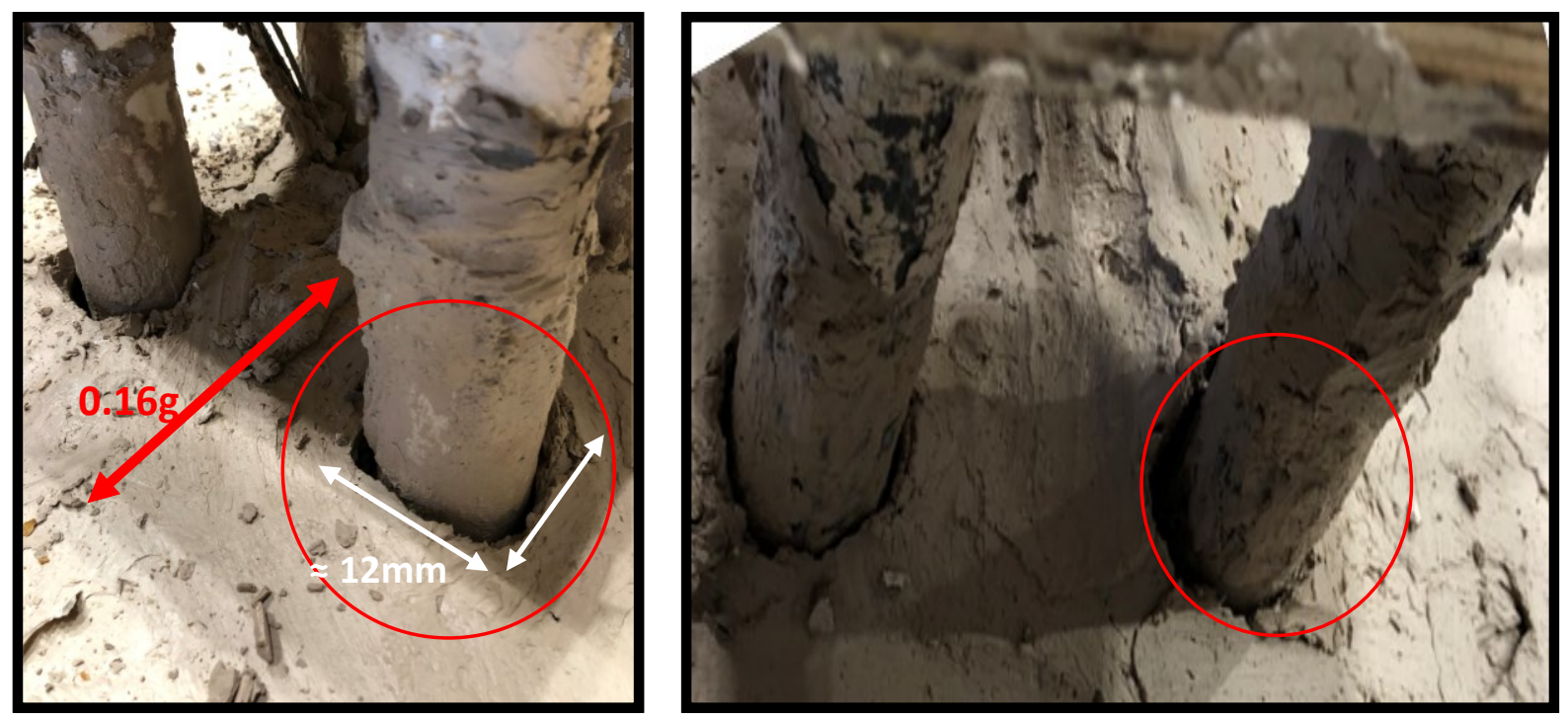

Figure 6.7: Development of gapping around model piles; Aluminium (left) and CFRP (right)

\subsection{Bending Moment of Pile Shaft and Pile Cap Deflection}

The lateral capacity of a rigid pile is typically controlled by the resistance provided by the soil and its response along the pile shaft, which is dominated by the failure of soil adjacent to the pile. 
Alternatively, the failure mechanism of a flexible pile involves the formation of a plastic hinge within the pile, and its lateral capacity is influenced by both the flexural stiffness of the pile and the soil resistance along the deflected length of the pile. Therefore, it is essential to verify the straining actions occurring in piles during shaking which can be captured by mounting several strain gauges to provide a reliable method to record varying strains with time during shaking experiments, however, they only measure strains at discrete points along the pile. Therefore, a curve fitting method must be employed to establish the straining pattern along the entire length of the pile. The bending moment envelope is defined by the absolute peak strain at each gauge during the excitation. This means that the result is not equivalent to actual bending moment diagram at the time step when the peak strain is recorded. As stated by the theory of elasticity and Hooke's law, the generated moment in the pile section is a function of the recorded strain in the strain gauges (Timoshenko, 1940), referring to the following expression:

$$
M=\frac{2 E_{p} I_{p}}{D} \times \varepsilon
$$

where, $E_{\mathrm{p}}$ is the modulus of elasticity, $I_{p}$ is defined as the cross-sectional moment of inertia, $D$ is the outer diameter of the pile, and $\varepsilon$ is defined as the recorded strain in the strain gauges. Figure 6.8 illustrates bending moment envelopes along the pile shaft for both the FRP pile groups and aluminium piles at shaking events of $\mathrm{K} 5$ and $\mathrm{K} 20$. As expected, the maximum bending moment in all pile models occurred at the base. It is critical to explain that the trends can clearly illustrate no domination by inertial forces from the superstructure masses due to lightly loaded piles, therefore, pile response was influenced by the kinematic forces from the soil that resulted the larger stresses in the piles as the intensity of the earthquake increased. 
Nevertheless, the distribution of the moment amplitude along the FRP pile shafts demonstrate similar trend with slightly greater bending moments on CFRP piles compared to GFRP piles along the shaft due to higher flexural stiffness (EI). However., traditional aluminium piles have reached much larger moments close to the base up to the surface. This may suggest that the bending moment (i.e., especially close to the base) has been dominated due to intensification of kinematic interaction through pile-soil models.
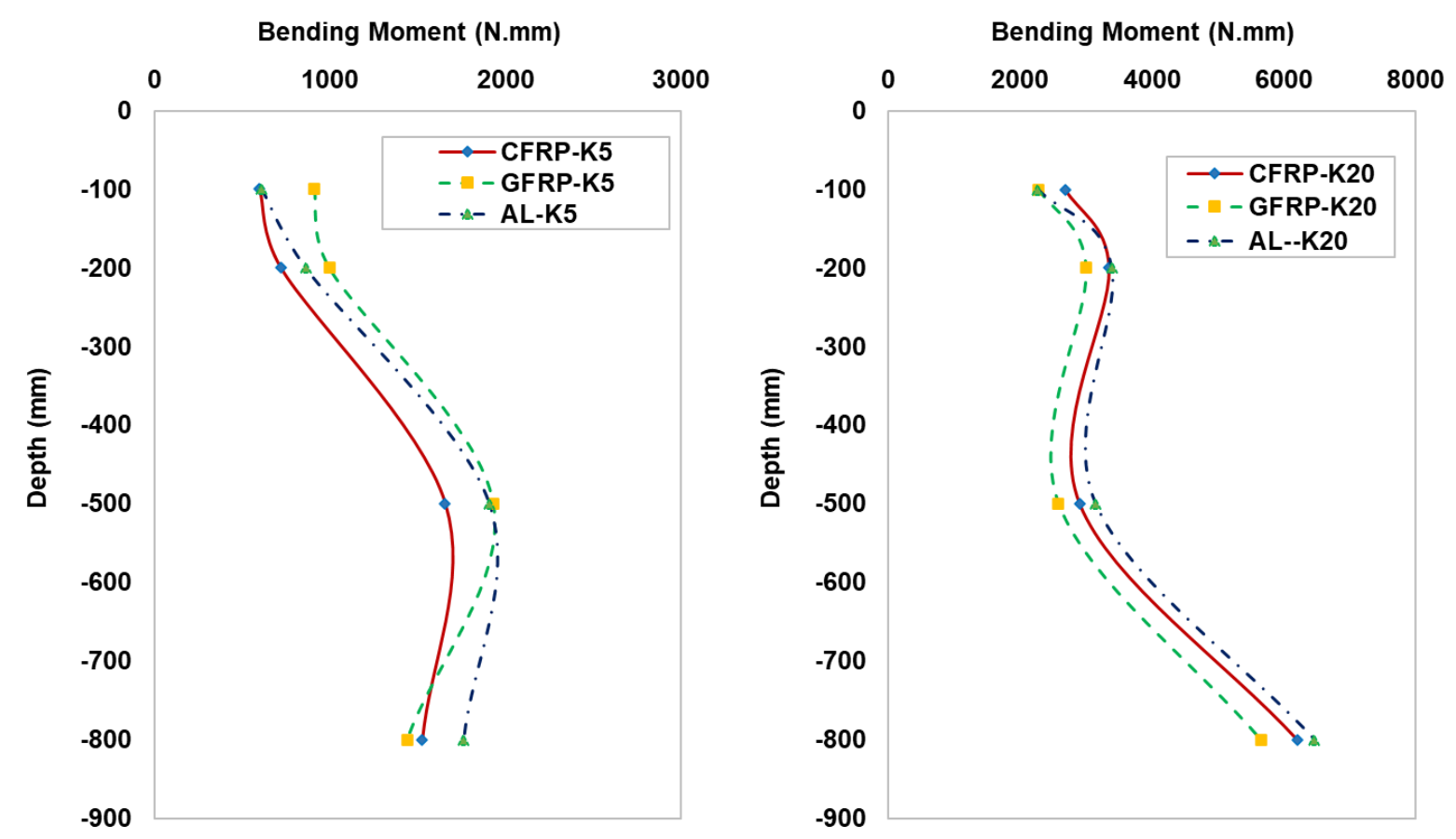

Figure 6.8: Recorded bending moment distribution along the piles at K5 and K20

To further validate the interaction effects, Figure 6.9 shows lateral deflections of the pile caps for all model piles during K20 shaking event. The lateral deflection indicates any movement at the cap; and the result revealed similar lateral deflections for the CFRP and aluminium models pile caps, while GFRP experienced slightly less deflection and residual displacement as the result of strong shaking (K20). This can be great indicator of material wave propagation and additionally 
it may be attributed to inertial interaction that impacted the response of the end-bearing aluminium group piles near the surface and its pile head.

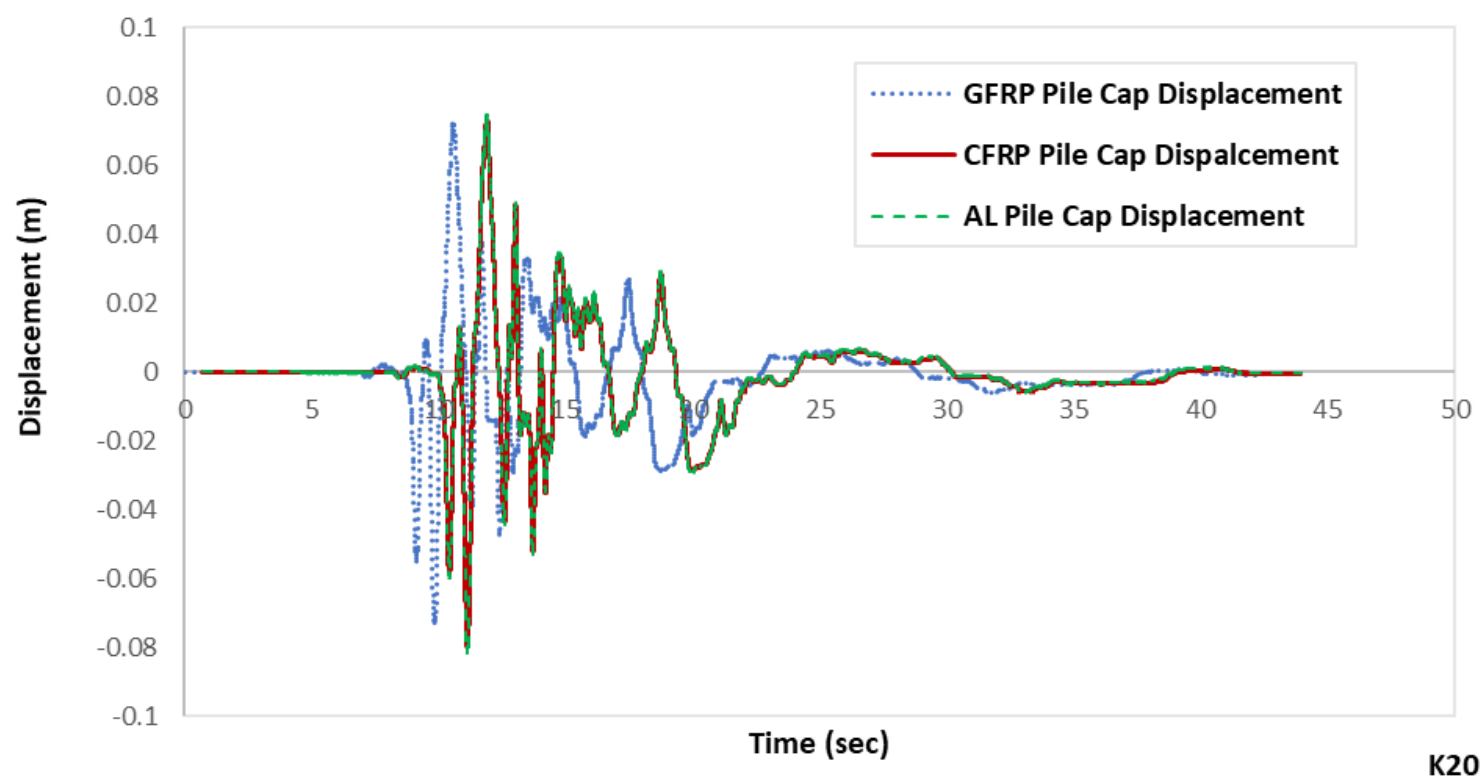

Figure 6.9: Pile head/cap deflection at K20

\subsection{Summary}

A data set was generated from these shake table tests to understand the seismic response of hollow FRP piles (Carbon and GFRP) in cohesive soils. The soil-pile interaction (SPI) in soft clay deposits is a complex process involving inertial interaction between structure and pile foundation, kinematic interaction between piles and soils, soil amplification and the non-linear response of soils during strong earthquake motions. Results discussed in this chapter have shown the following conclusions:

- Soft clay deposits were shown to particularly amplify shaking, though surface motions were attenuated during very strong shakings due to soil non-linearity and stiffness degradation. 
- The earthquake characteristics, soil properties, and soil-pile-structure interaction (SPSI) were found as critical factors that can impact the seismic excitation experienced by the pile cap and the structures.

- The seismic response of the soil deviated the response of FRP piles from that of free-field motions at a period of 0.05-1 seconds, corresponding to the frequency range of 1-20 Hz.

- The frequency content of the amplified motions appeared to be consistent among the model pile groups. Therefore, the soil-pile interaction has not deviated the frequency range of seismic motions recorded for both the pile systems and only amplified the input motion.

- Comparing the response of the FRP piles, the result illustrated that CFRP piles have reached higher motion response in base which in turn induced larger bending moment and hence, lower response closer to surface. Alternatively, aluminium piles have reflected significant kinematic forces and seismic waves on the cap and structure which this result can also further underscore the importance of pile ductility and hence the kinematic pilesoil interaction on seismic response of pile foundations in seismic prone areas. 


\section{Chapter 7: Seismic Performance of Fibre-Reinforced Polymer (FRP) Model Piles in Soft Clay}

\subsection{Introduction}

The observed damage and subsequent processing of strong ground motion recordings obtained on soft soil deposits during past earthquakes have revealed the significant importance of seismic site response and soil-pile-structure interaction (SPSI) role in the analysis and design of foundations and structures. Geotechnical engineers have long recognized this role, and many studies have been focused on several aspects of the topic in the past decades. Under seismic excitation, a pile may experience kinematic interaction resulted from the inability of a stiff foundation in or on the softer soil to move in the identical manner as the free field motion of the site. The main factors contributing to the kinematic interaction include the foundation rigidity and embedment depth effects, and incoherency. Therefore, the behaviour of foundations during earthquakes is often dictated by the response of the supporting soil and its kinematic interaction. On the other hand, inertial interactions, as another dominating mechanism, may also alter the vibrational characteristics of structures which can create base shear and moment effects at the foundation level resulting in relative displacement between the foundation and the soil. Evaluating these interactions is crucial in the seismic design of piles and pile-supported structures during earthquakes.

Several studies were conducted on the role of soil-pile interaction (SPI) in performance of traditional pile materials (e.g., steel, timber, concrete) using aluminium pipe and/ or other modified pile material (e.g., Tao et al.,1998; Maugeri et al., 2000; Chau et al., 2009; Ling et al., 2013; Tang et al., 2014; Durante, 2016; Dong et al., 2019; Azizkandi et al., 2020). However, there is a lack of 
detailed experimental seismic investigation on Fibre-reinforced polymer (FRP) piles. This study is specifically focused on broad aspect of newly introduced hollow fibre-reinforced polymer piles as an alternative in many geotechnical applications. FRP materials have advantages over conventional pile materials because of their durability and corrosion resistance. Therefore, the engineering application of FRP may present competitive alternatives to conventional pile materials due to the accelerated degradation of these conventional materials in aggressive environments. FRP composite piling is commonly used as waterfront barriers, fender piles, and bearing piles for

light structures (Iskander and Hassan 1998). FRP composite piles are gaining wide acceptance in the geotechnical industry, however, because of lack of studies on seismic response of these piles, it has become a challenging foundation alternative in seismic prone zones.

This chapter presents the result of shaking test program on hollow frictional FRP group piles embedded in soft clay. Due to large volume of the experimental results, only representative details are reported. Analyses were conducted to investigate free-field site response, effects of shaking level, soil-pile interaction, influence of pile cap on performance of model piles, and effect of material characteristics on seismic response of these model piles.

\subsection{Experimental Results}

A total of eight accelerometers were implemented to capture seismic response of the model frictional piles in soft cohesive soils during shaking. These accelerometers were distributed within the soil profile in free field and mounted along the shaft of a model pile per group as well as their pile caps. Figure 7.1 presents schematic drawing that specifies the location of the accelerometers with their allocated subscription along with additional instruments used to monitor the shaking 
system during variety of excitations. Sample acceleration times histories for the free field motion (A4) and corresponding pile motions along the shaft for the GFRP at surface and base (A2 and A5) during strong shaking of Kobe 20 are shown in Figure 7.7. The key features considered are divergence between the acceleration time histories recorded along each model pile and in the free field. Additionally, the recorded maximum accelerations in terms of ' $\mathrm{g}$ ' at each accelerometer for free field motions as well as all model piles are summarized in Table 7.1. The distribution of the maximum acceleration within soil profile in free field along with peak acceleration distributions throughout the length of piles at different seismic events are also shown in Figure 7.3. The overall distribution of acceleration shows a similar variation order under different seismic intensities, where the peak acceleration at the base of the model piles shows considerable rise which enhances with increase in acceleration of applied excitations.

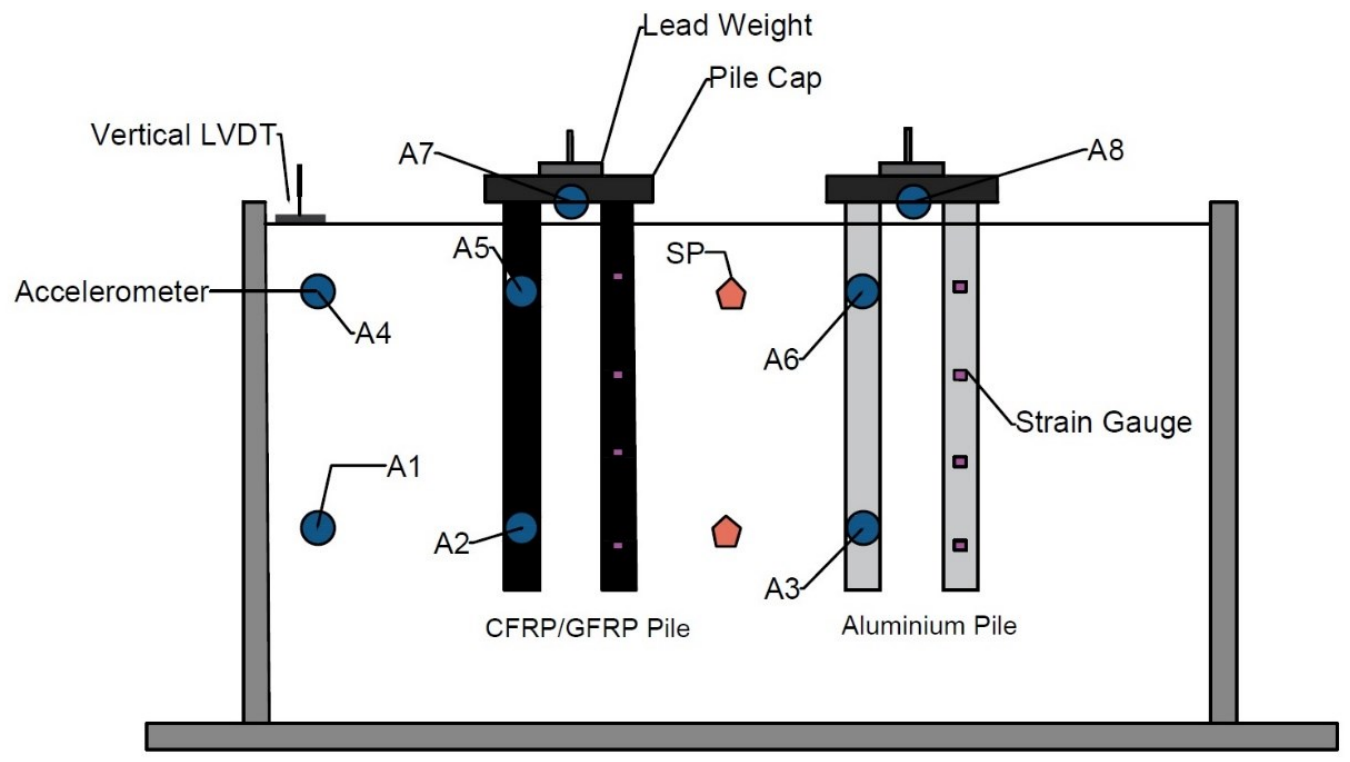

Figure 7.1: Instruments configuration in laminar container 

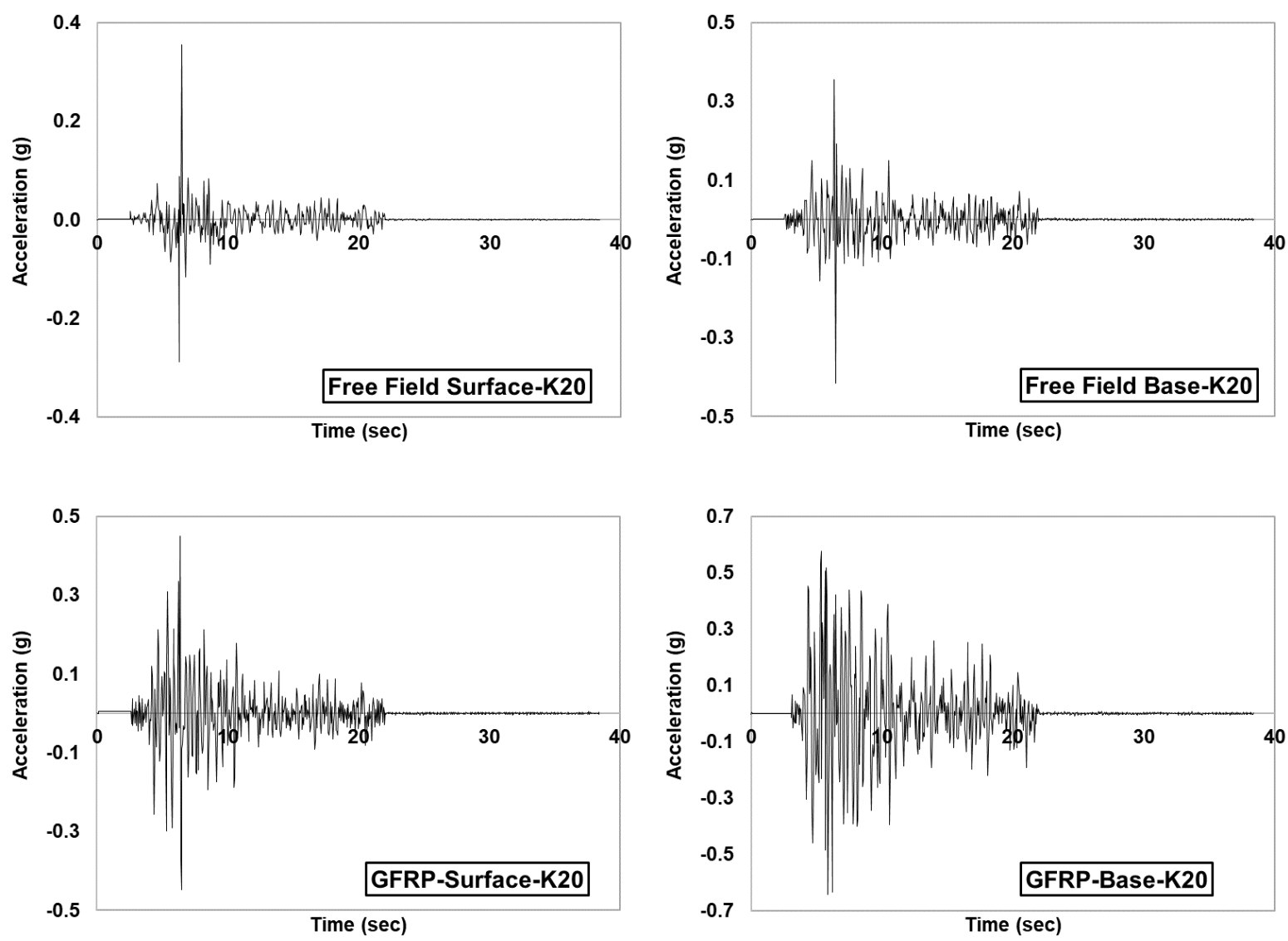

Figure 7.2: Acceleration time histories free field and GFRP at K20 shaking

Table 7.1: Recorded Peak accelerations for different excitations in all experimental events

\begin{tabular}{|c|c|c|c|c|c|c|c|c|c|c|}
\hline $\begin{array}{c}\text { Model } \\
\text { Piles }\end{array}$ & $\begin{array}{l}\text { Input } \\
\text { events }\end{array}$ & $\begin{array}{c}\text { Input } \\
\text { motions } \\
\text { (g) }\end{array}$ & $\begin{array}{l}\text { A1 } \\
\text { (g) }\end{array}$ & $\begin{array}{l}\text { A2 } \\
\text { (g) }\end{array}$ & $\begin{array}{l}\text { A3 } \\
\text { (g) }\end{array}$ & $\begin{array}{l}\text { A4 } \\
\text { (g) }\end{array}$ & $\begin{array}{l}\text { A5 } \\
\text { (g) }\end{array}$ & $\begin{array}{l}\text { A6 } \\
\text { (g) }\end{array}$ & $\begin{array}{l}\text { A7 } \\
\text { (g) }\end{array}$ & $\begin{array}{l}\text { A8 } \\
\text { (g) }\end{array}$ \\
\hline \multirow{6}{*}{$\begin{array}{c}\text { CFRP } \\
\text { and } \\
\text { AL } \\
\text { (T3) }\end{array}$} & $\mathbf{0 5 0}$ & 0.020 & 0.030 & 0.099 & 0.075 & 0.033 & 0.065 & 0.078 & 0.027 & 0.028 \\
\hline & 0100 & 0.041 & 0.068 & 0.109 & 0.081 & 0.065 & 0.087 & 0.070 & 0.080 & 0.145 \\
\hline & 0200 & 0.082 & 0.110 & 0.188 & 0.200 & 0.110 & 0.168 & 0.200 & 0.120 & 0.251 \\
\hline & K5 & 0.041 & 0.080 & 0.217 & 0.232 & 0.080 & 0.168 & 0.162 & 0.150 & 0.240 \\
\hline & K10 & 0.082 & 0.152 & 0.367 & 0.378 & 0.149 & 0.291 & 0.273 & 0.344 & 0.467 \\
\hline & K20 & 0.164 & 0.313 & 0.422 & 0.479 & 0.278 & 0.345 & 0.437 & 0.466 & 0.699 \\
\hline \multirow{6}{*}{$\begin{array}{c}\text { GFRP } \\
\text { and } \\
\text { AL } \\
\text { (T4) }\end{array}$} & & 0.020 & 0.036 & 0.110 & 0.100 & 0.035 & 0.070 & 0.078 & 0.028 & 0.029 \\
\hline & O100 & 0.041 & 0.070 & 0.150 & 0.150 & 0.070 & 0.100 & 0.115 & 0.150 & 0.160 \\
\hline & $\mathbf{O 2 0 0}$ & 0.082 & 0.137 & 0.250 & 0.250 & 0.129 & 0.248 & 0.297 & 0.160 & 0.290 \\
\hline & K5 & 0.041 & 0.081 & 0.250 & 0.210 & 0.079 & 0.150 & 0.175 & 0.200 & 0.241 \\
\hline & K10 & 0.082 & 0.147 & 0.467 & 0.241 & 0.136 & 0.190 & 0.229 & 0.269 & 0.348 \\
\hline & K20 & 0.164 & 0.311 & 0.510 & 0.418 & 0.272 & 0.449 & 0.497 & 0.549 & 0.697 \\
\hline
\end{tabular}


The peak acceleration within the soil profile (A1 and A4) indicated development of similar motions along the soil profile in weaker seismic events (e.g., O50 and O100). As the intensity of base excitation increases, a reduction in peak acceleration can be detected toward the surface in free field response, and comparably a similar pattern was noticed for FRP piles near the surface. As it will be discussed later, this reduction in free field motion could be attributed to induction of higher strain in soft soil deposits, and further complications due to the nonlinear soil response during stronger input motions. Nevertheless, the maximum accelerations within the soil (free field) for all applied excitations were significantly less than those measured on piles at the same depth. This difference in peak ground motions underscores the importance of considering soil-pile interaction impacts on foundation input motions in seismic prone areas.

In general, the model piles have performed within similar range of acceleration at particular depth although the significance of the response varied as intensity of input motion changed. The FRP piles experienced higher acceleration at the toe and followed by a drop in peak acceleration near the surface in all shaking events. This behaviour was similarly observed in response motion of Aluminium piles along the shaft. Although, there was significantly higher maximum acceleration felt at the aluminium pile cap compared to the CFRP/GFRP group pile caps. This also means that superstructures rested on aluminium group piles may have suffered considerably greater acceleration compared to loaded bar mass structure resting over the CFRP and GFRP piles. This may be attributed to material characteristics (i.e., flexural stiffness) of the model piles which highlights their response, and hence higher deviation between the foundation and free field motions resulted from kinematic soil-pile interaction. The higher peak accelerations in the aluminium pile cap may also cause further problems such as generation of the base shear and 
rocking that consequently can appear as deformation and perhaps rotation of the foundation relative to the free field.
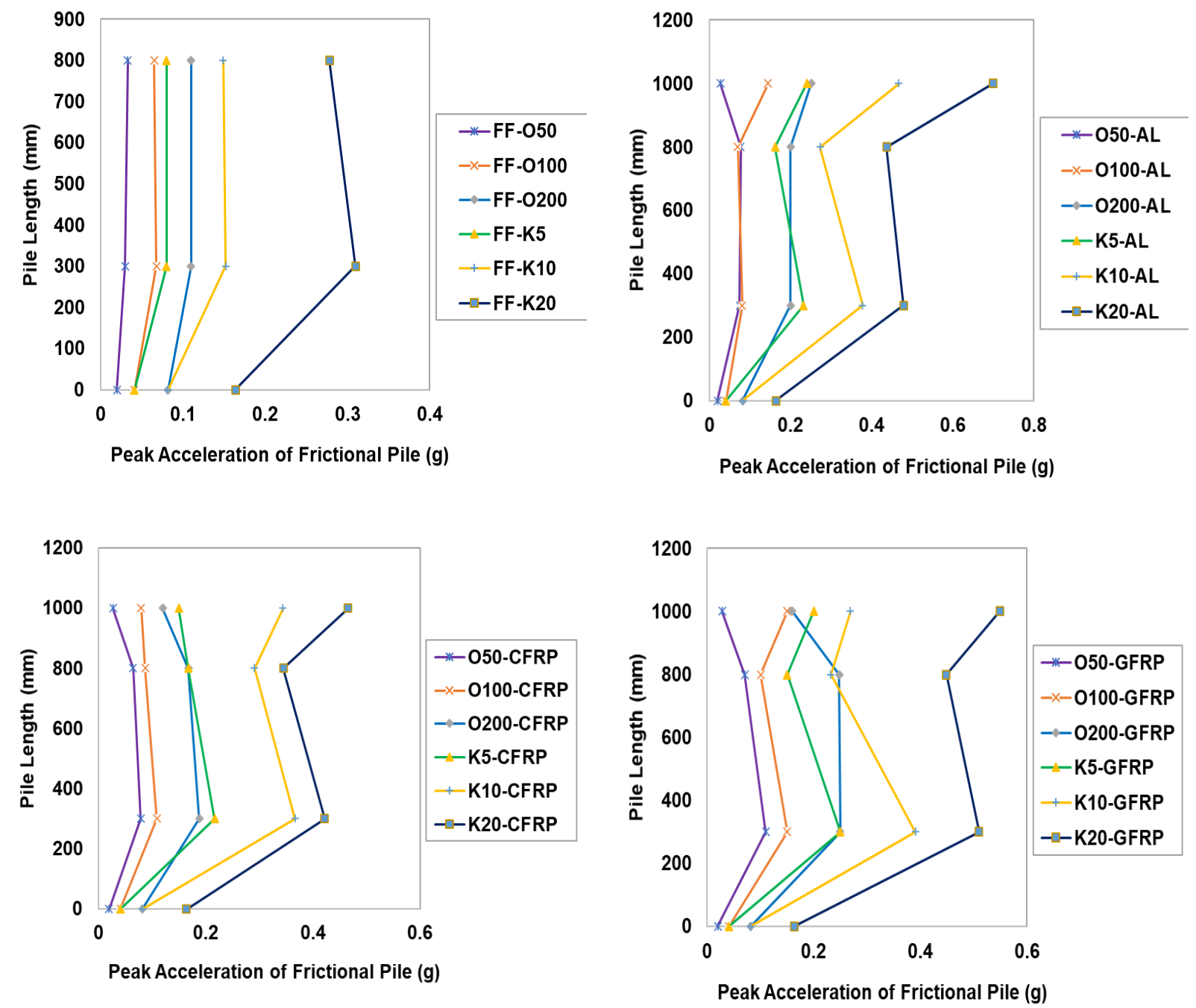

Figure 7.3: Recorded maximum acceleration of free field and model piles at different seismic loading (FF, free field; AL, aluminium pile; CFRP, carbon FRP, GFRP, glass FRP) 


\subsection{Site Effect and Soil Amplification}

An extensive parametric study has been undertaken to characterize the response behaviour of soft clay in varying levels of earthquake excitation. These analyses were performed by assessing two excitations with specific level of duration and frequency characteristics to represent moderate to large earthquakes. Soft clay can amplify the seismic input motion through the soil profile toward the surface and as the intensity of excitation increases, the surface response may illustrate the nonlinearity of soil and stiffness degradation (Idriss, 1990). It is also postulated that such motions may even induce shear failure in the soil deposit. The model soil container is intended to approximate site response, which requires that ground motions be identical at all points on any horizontal plane. To evaluate the model soil response, the acceleration response spectra from allocated accelerometer within free field boundary for two excitations of O200 and K20 were compared in Figure 7.4. For the moderate shaking of O200, it can be noted that soil motion was amplified at certain point with minor attenuation toward the surface. At higher levels of acceleration such as K20, the soil nonlinearity seems to lead to progressive softening and incipient failure of soft soil resulting in (a) de-amplification of the high frequency components of motion

and peak surface accelerations, (b) a partial shift in spectral response energy to longer period of motions, and (c) large ground displacements. 

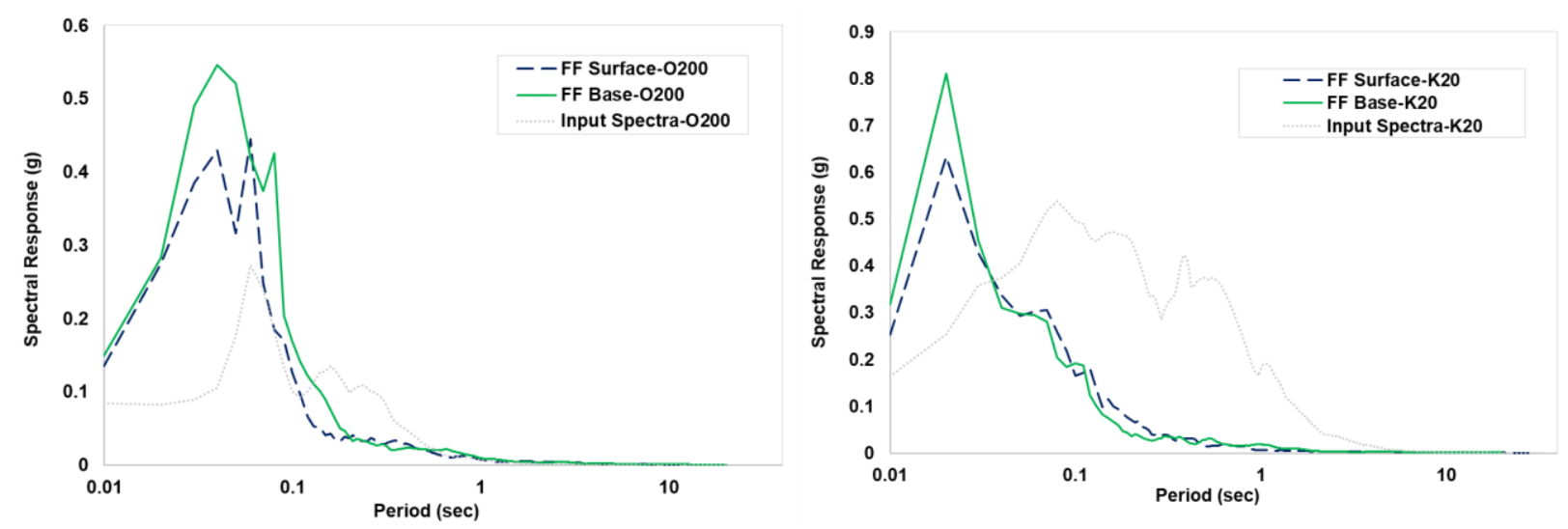

Figure 7.4: Free field spectral acceleration during the $\mathrm{O} 200$ and K20 shaking events

\subsection{Spectral Acceleration on Model Piles}

The embedded frictional model piles (i.e., CFRP, GFRP, and aluminium) in synthetic soft clay were subjected to several excitations from low to strong ground motions. Figure 7.5 demonstrates the acceleration response behaviour of model group piles at moderate and strong shaking events of O200 and K20. At moderate shaking, FRP piles (i.e., carbon and glass) demonstrated closer acceleration trend with aluminium group piles within frequency range of 10 to $40 \mathrm{~Hz}$, while higher amplification was sensed near the surface of aluminium piles. Though, at lower sections of the pile, amplification occurred due to the kinematic forces from the soil which produced larger stresses in these piles. Accordingly, a reduction in amplitude of the foundation transitional motion occurs, while foundation rocking motion is introduced as a result. This outcome is correspondingly resulted in similar tendency at the intense motion of K20.

To investigate the impact of pile characteristics on soil-pile interaction, a spectral ratio was established using the pile/soil response ratios (RRS) to further explore the kinematic interaction for different piles (Figure 7.6). For FRP piles, the maximum values of RRS were recorded beneath 
the pile cap during all excitations. This value, however, was slightly higher for the aluminium piles in both excitations of $\mathrm{O} 200$ and $\mathrm{K} 20$, though the vibration frequencies seem to be consistent for all the piles at frequency level of 10-100 Hz. Additionally, FRP and aluminium piles appear to respond proportionally similar at the base with the RRS value of around 3.0 and at surface with a value of about 1.0-1.2. This explains that piles response behaviour at surface are attributed to strong soil-pile interaction and strong nonlinearity of upper sections of soft clay in laminar model container.
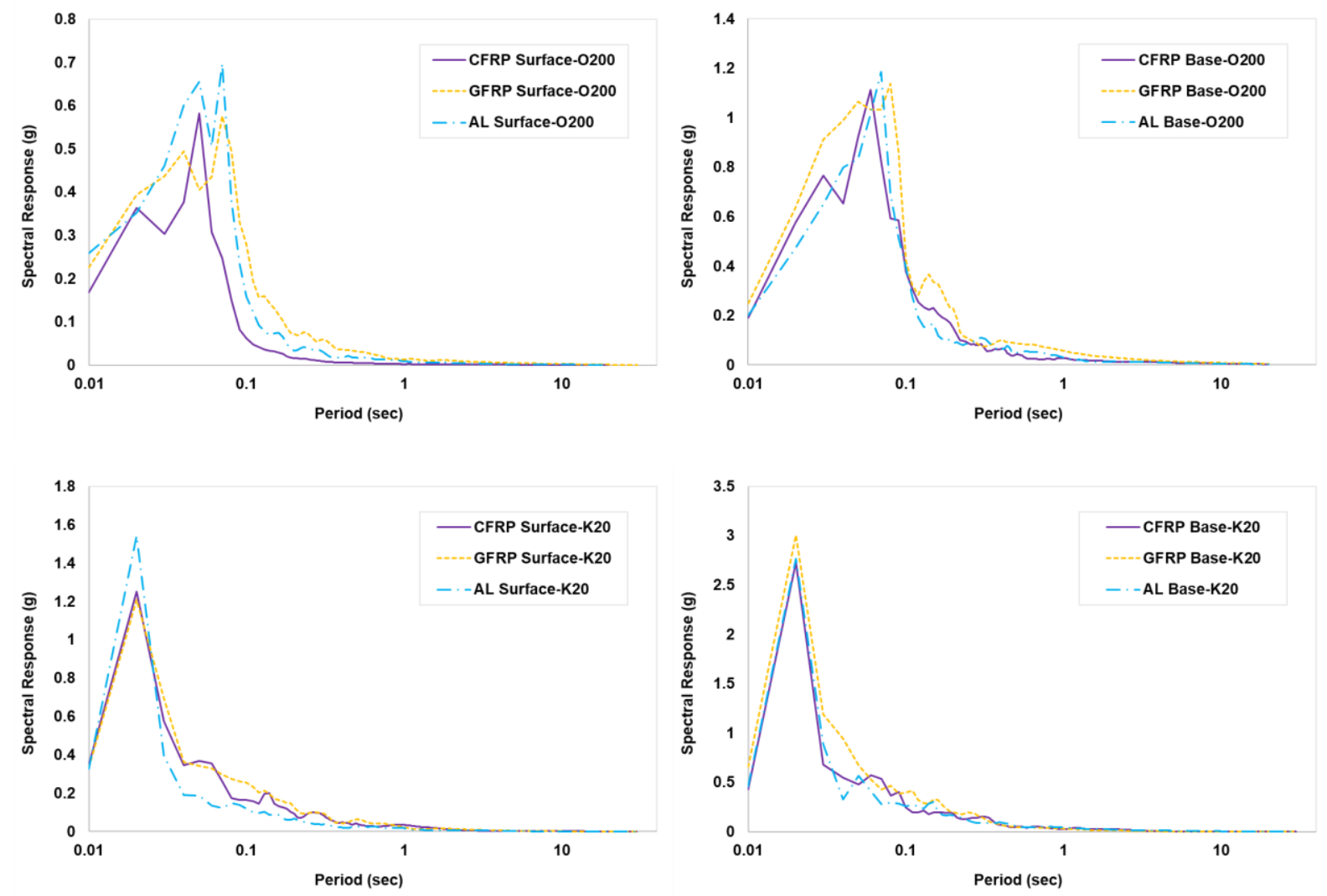

7.5: Spectral acceleration of model piles at $\mathrm{O} 200$ and $\mathrm{K} 20$ excitations 

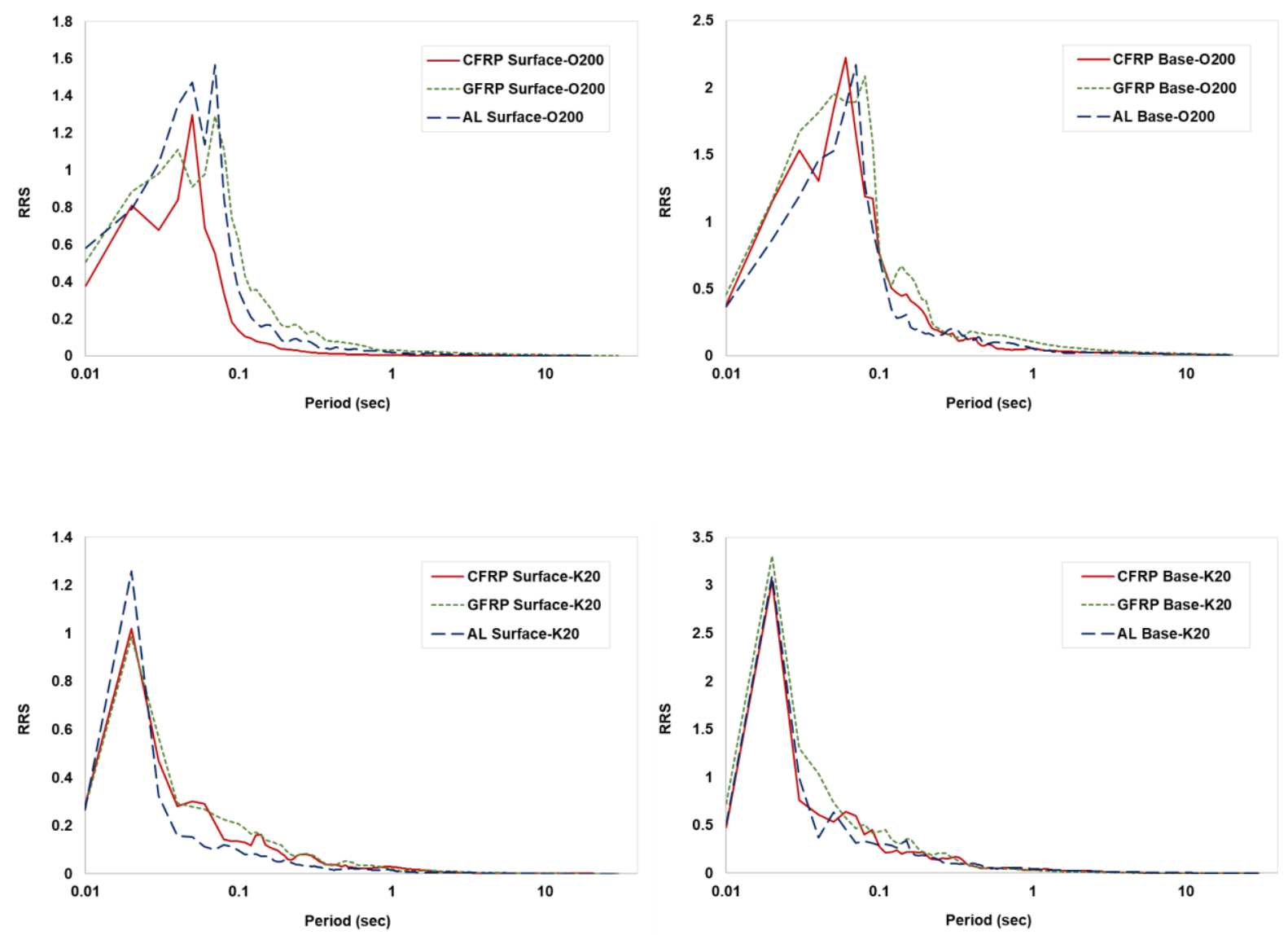

Figure 7.6: Pile/soil response spectral ratio (RRS) for all model piles during O200 and K20 shaking events

\subsection{Effect of SPI on Frictional Pile Cap Response}

Influence of the underlying soil on seismic response of the pile cap and resting structure can be ignored if the ground is stiff enough, and the structure can be analysed considering fixed-base conditions. However, the same cap and structure behaves differently when it is constructed on soft soil deposit. Earthquake characteristics, travel path, local soil properties, and soil-structure interaction are the factors affecting the seismic excitation experienced by piles and its structures. Consequently, the type of pile materials used in seismic prone zones can determine and alter the response of pile cap and its supporting structure which conversely can affect the pile foundation 
response. The response spectra of pile caps on model piles are presented in Figure 7.7. The results illustrate that material type and its characteristics can alter the response of the pile caps with increase in intensity of excitations (O200 to K20). Pile caps response in O200 events (i.e., moderate shaking) versus free field response at surface reveals major attenuation and wave scattering which were impacted by the interaction of the model piles with the surrounding soil. However, at strong input motion of K20, pile caps were tolerated by the site response and accordingly, rocking motions were experienced more evidently at the cap and surface response specifically aluminium pile caps, which this can subsequently disclose higher bending moment for aluminium piles at surface in comparison to FRPs due to higher flexural rigidity and stiffness and hence greater impact toward kinematic soil-pile interaction.
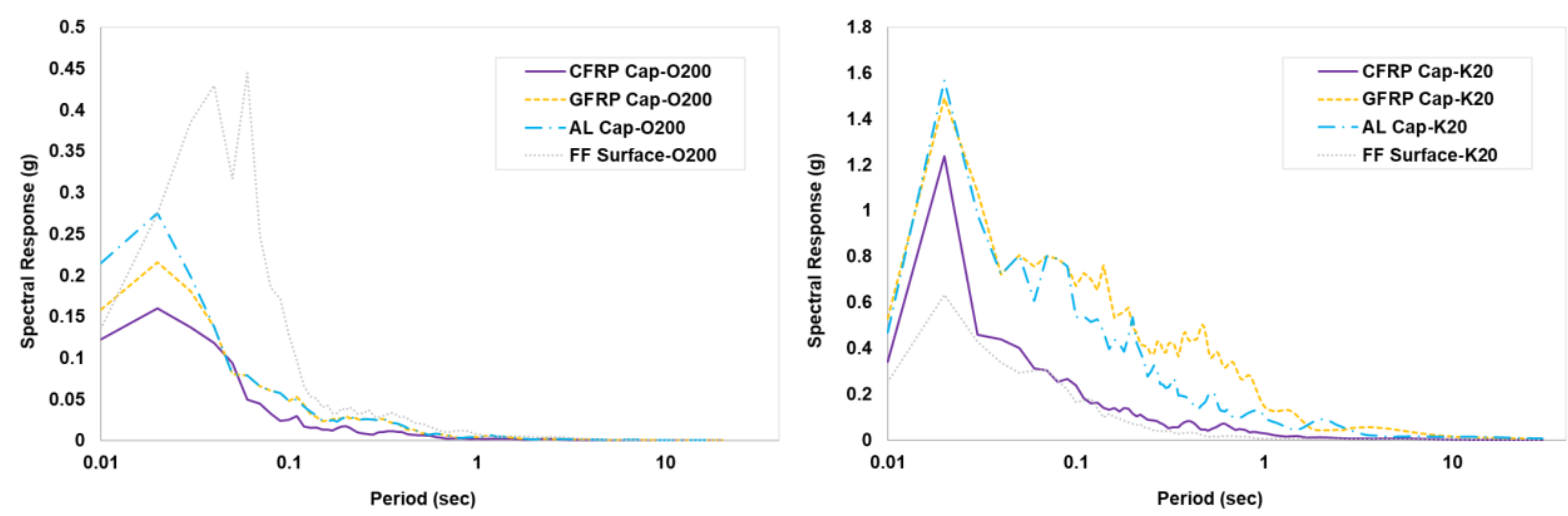

Figure 7.7: Pile cap reponse spectra at $\mathrm{O} 200$ and K20 shaking events for all model piles

\subsection{Impact of Flexural Stiffness on Seismic Performance of Piles}

The induced bending moment profile along the pile shaft under kinematic and/or any inertial interactions is the key parameter to ensure structural integrity of the foundation under seismic loading. Additionally, flexural stiffness of piles (EI) can play an essential role in induced bending 
moments of piles under seismic loading. To examine the role of flexural stiffness on piles' response, FRP and aluminium piles with different EI values were analysed while all other parameters including the model geometry were remained constant. The higher EI values, corresponding to higher stiffness, were measured for aluminium piles while FRP model piles recorded relatively lower EI values (specifically GFRP). The effects of EI value on pile cap displacement and induced bending moments were examined using the recorded strain records and plotted in Figures 7.8 and 7.9, respectively. The result at K20 excitation level validates that GFRP experienced less displacement during the motion followed by CFRP, and then aluminium piles with highest deformation which has accordingly attained higher acceleration response at surface. Also, based on the envelopes of the peak bending moments, the remarked results show considerable moment in mid-length of all model piles as larger bending induced due to stronger input acceleration (i.e., K20). This means piles have experienced larger kinematic forces in midsections prior to the surface of piles. As expected, FRP model piles have shown larger ductility along their profile which means the stiffer aluminium piles experienced 1.1 to 1.5 times larger maximum bending moment compared to the hollow FRP model piles. Based on the observed bending moment profiles along the pile shafts, designing the short piles just for lateral bearing capacity may not always be adequate in seismic prone regions from seismic design perspective, in particular when considering kinematic soil-pile interaction. One possible solution can be increasing diameter of the pile shaft to improve its moment capacity. 

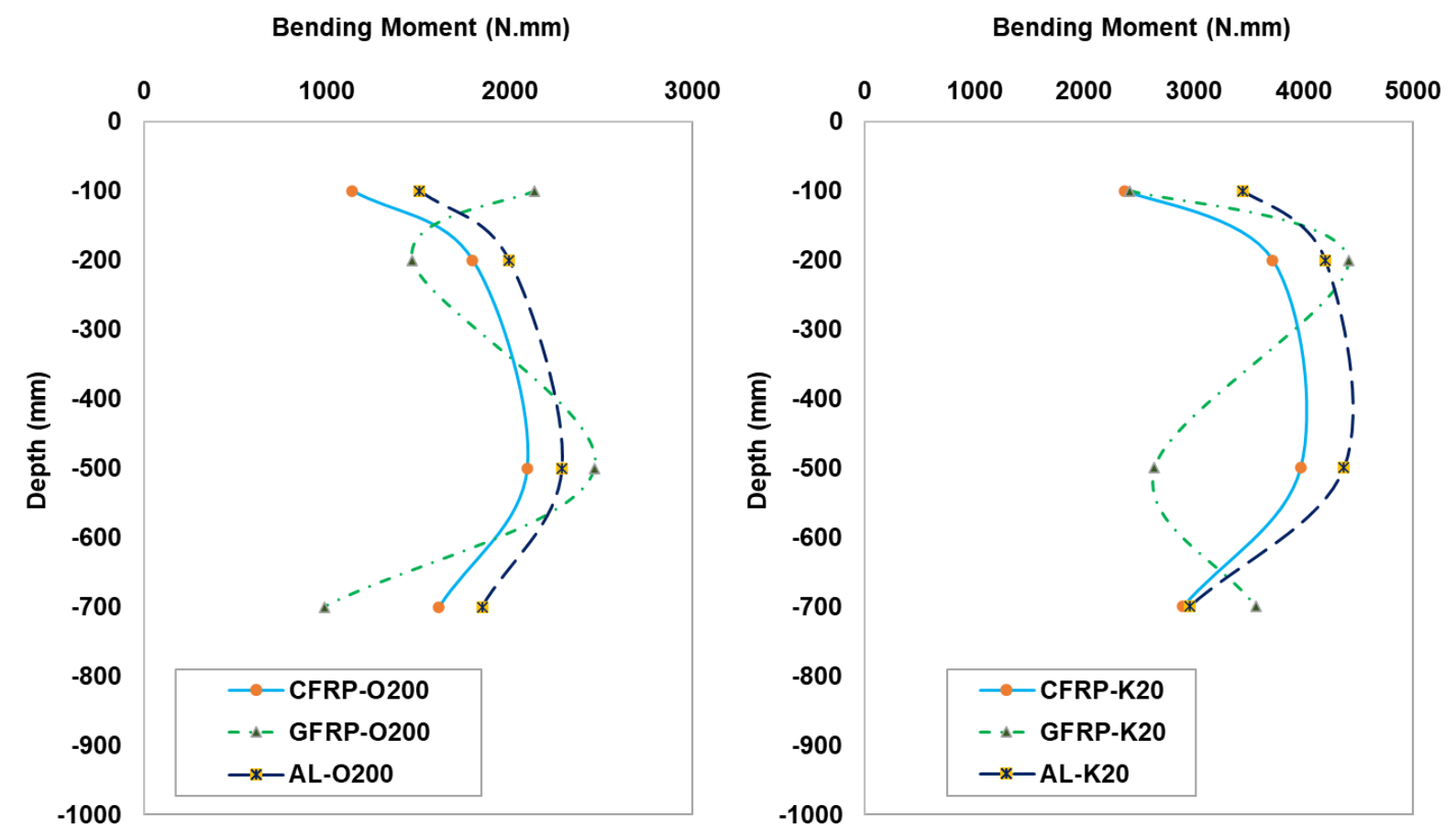

Figure 7.8: Recorded bending moment distribution along the piles at $\mathrm{O} 200$ and K20

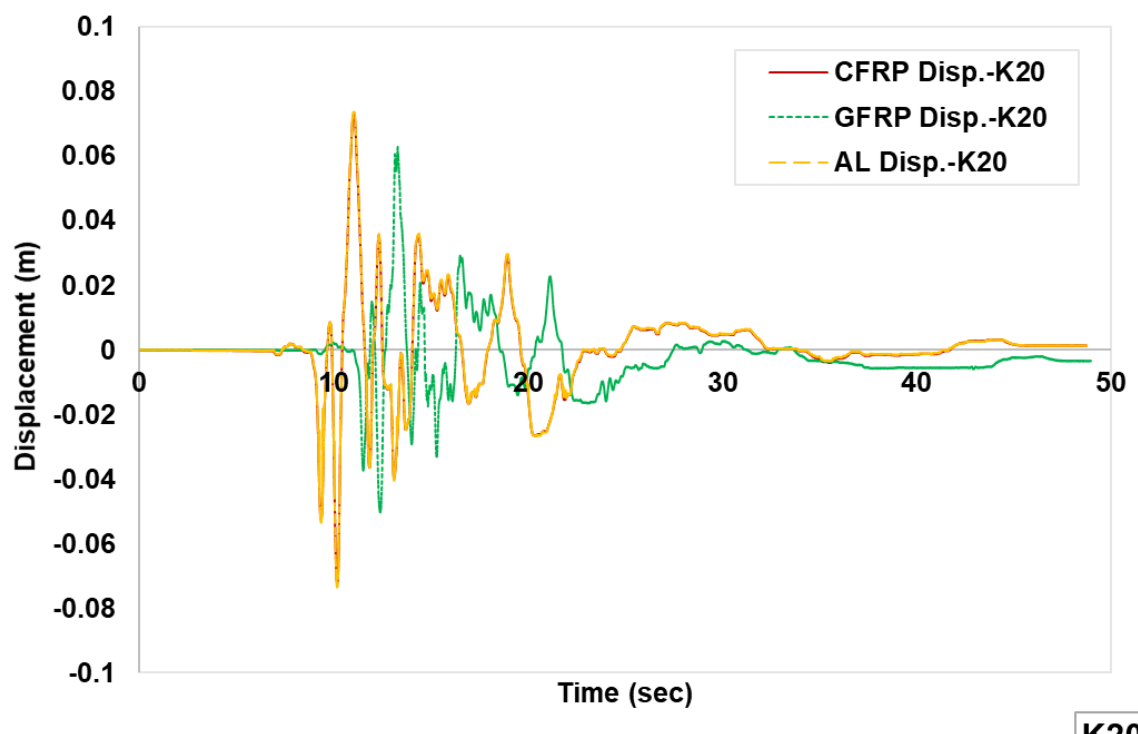

Figure 7.9: Pile head/cap displacement at K20 


\subsection{Seismic Response of Frictional vs End-bearing Model Piles in Soft Clay}

It has been well understood that rocking motion of foundations partially plays a major role in inertial soil-pile-structure interaction. Therefore, to further clarify structure-foundation motion in conjunction with translational motion and period elongation, Figure 7.10 reveals the impact of pile type (i.e., end-bearing and frictional) on seismic response of pile caps under fixed bar mass when underwent same excitation. During both the Kobe and Ottawa earthquake vents, the pile cap sitting over frictional FRP piles experienced greater level of amplification compared to the end-bearing pile which can consequently lead to higher possible structural damage. This could be related to the presence of soft clay underneath the frictional piles and its effect on amplifying the input motion for frictional piles. The period of vibration was also different for the two pile groups with different load transfer mechanism (i.e., end-bearing vs frictional). The end-bearing piles demonstrated a higher period of vibration relative to frictional piles possibly due to interface gapping of soft clay (stiffness degradation) around end-bearing piles.
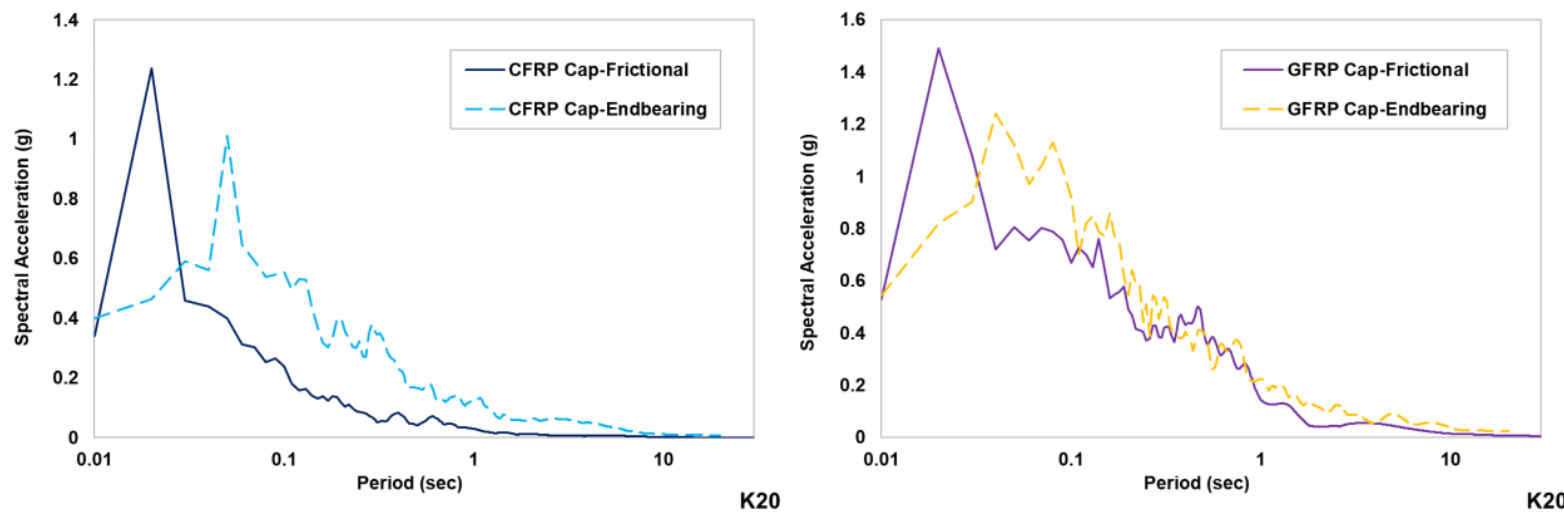

Figure 7.10: Pile cap reponse spectra at O200 (left) and K20 (right) shaking events for frictional and endbearing FRP model piles 


\subsection{Summary}

A series of shaking model tests was conducted to study the seismic site response and seismic soilFRP pile interaction (SPI) for model piles embedded in soft clay profiles. Several earthquake-like shaking events were applied to the laminar container model to investigate linear and nonlinear soil behaviour. As SPI covers a wide range of topics including static and dynamic problems, parametric study has been undertaken to characterize the response behaviour of model piles in synthetic soft clay medium under varying levels of earthquake excitation. The input motions were adopted from the 2010 Val-des-Bois earthquake and another stronger shaking from the 1995 Kobe earthquake. These analyses were performed using input motions with different peak acceleration at specific level of duration and frequency characteristics to represent low to high earthquake shaking levels. This chapter presented the results of a series of scaled shaking table tests conducted on eight frictional hollow FRP piles (carbon and glass polymers) as well as four conventional aluminium piles. The following is a summary of observations made from the results discussed in this chapter:

- Generally, the dynamic response of frictional pile foundations during seismic loading considerably depends on the performance of soft clay surrounding them along with the intensity of bedrock (input) motion. Therefore, if there is no yielding of clay during the dynamic loading, then both the free field and pile foundations' acceleration response will be amplified relative to the input motion. However, when there is a yielding of clay during strong motion, the free field surface motion could be attenuated strongly. This behaviour alters the response of the pile models during such attenuated motions which depends on the motion received by the pile at their base (toe) levels and the excitation of the surrounding soil. 
- At higher levels of acceleration (such as K20) soil nonlinearity led to progressive softening and incipient failure of soft soil resulting in de-amplification of the of motion near surface, and partial shift in spectral response energy to longer period of motions.

- Response of model piles, specifically frictional FRPs, were fairly filtered near to the surface as larger response was carried out at the base in all modes during low-to-moderate excitations, followed by lower amplification at the pile caps (e.g., O200 pile cap response). This outcome was attributed to high flexibility FRP material and high strength-to-weight ratio.

- The frequency content of the amplified motion appeared to be consistent at the corresponding range of $10-100 \mathrm{~Hz}$ with partial shift in spectral response energy to longer period of motions at K20 excitation due to failure and gapping of soft clay (stiffness degradation).

- CFRP pile caps experienced lower amplitude of amplification in all excitations, therefore less energy due to kinematic forces has affected the piles and their pile cap/superstructure.

- Pile cap and structural vibrations tend to impose large bending moments at aluminium piles with large difference between FRP piles bending moments indicating strong effect of pilesoil-pile interaction. 


\section{Chapter 8: Summary, Conclusions and Recommendations}

\subsection{Research Summary and Findings}

This dissertation described the results of a study on the seismic response of FRP model pile foundations embedded in liquefying sand and soft clay under several shakings. The research were divided into several components: (1) a series of shaking tests of FRP group and aluminium group piles in soft and liquefying soils; (2) a critical investigation of physical modeling techniques and limitations such as designing the soil mix and laminar soil container; (3) analysis of soil response and soil-pile interaction along the soil profile and pile shaft, pile bending moment, pile head deformation response, and additionally change in pore water pressure of liquefiable soil; and (4) evaluation of pile foundation type (i.e., end-bearing and frictional) and the influence of kinematic interaction on their response. The following sections summarize the principal finding of these studies considering the results in the context of practice and provide recommendations for future study.

\subsubsection{Shaking Table Modeling}

Principles of scale model similitude were used to derive a set of model scaling relationships that were used in a method of "implied prototypes" to relate model and prototype behaviour. In the conducted shaking table tests in this thesis, soil-pile models were physically simulated with geometric scaling factor of 1:10. A specialized laminar container with dimensions of 1.0 meter by 1.0 meter and a height of 1 meter was designed to allow the soil to respond in the same fashion as the free field soil response in order to minimize the boundary effects. Accordingly, these shaking table tests were performed by adaptation of the consistent soil properties, equivalent bar mass 
superstructure, identical input motions, and similar experimental setup in order to provide a clear comparison among the two types of the pile groups in terms of the axial load carrying system; frictional and end-bearing piles, to assess the seismic performance of FRP piles in comparison to traditional piles (e.g., aluminium). Each soil-pile model was subjected to real earthquake input motions including the 2010 Val-des-Monts and the 1995 Kobe Earthquake. The shaking tests were conducted by monitoring and controlling laminar shear box to distinguish the influential parameters in response of soil and pile. The improved physical modelling techniques in this study such as designing the soil preparation and laminar soil container were explained in detail, which can be used by other researchers to accomplish and improve the simulation of the soil-pilestructure interaction of FRP piles in future $1 \mathrm{~g}$ shaking table tests.

\subsubsection{Description of Model Piles}

Model piles were divided to $2 \times 2$ model pile groups embedded in uniform liquefiable sand and/or synthetic soft clay, supporting a bar-mass system representing superstructure which was fixed to the pile cap. The hollow FRP piles were designed to be manufactured with commercially available carbon and glass FRP fabrics used for the reinforcement and retrofit of foundation and structural members. The raw material was used to manufacture the model piles. Aside from FRP piles, a traditional pile of prefabricated aluminium tube was selected based on the range of adequate modulus to be configured as a thin wall section to meet the EI criterion and follow the scaling rule. With respect to axial performance, calculations of driving stresses, static loads, and stresses under dynamic loading were found not to exceed the buckling load. Specific problems examined included design of hollow FRP piles where considering SPI and interface conditions is employed to advance efficient design procedures; and the performance of FRP piles under dynamic loading that exploits the understanding of SPI during shaking for FRP and traditional piles. 


\subsubsection{Soil Characterization}

Sand samples for shaking tests were prepared by raining (pluviation) at the surface of the laminar container and dispersed consistently to prepare homogenous soil medium. To form a sand profile with uniform density, two factors are of prime importance in this method: (i) the height of fall of the sand and (ii) the intensity of deposition (i.e., the weight deposited per unit area). Additionally, the uniformity of the soil samples was checked by monitoring the average relative density of the soil and monitoring the soil settlement at every shaking level (i.e., recorded level of soil sample).

A synthetic soft clay was selected as the soft cohesive soil medium with mixture ingredients of kaolinite, and bentonite powders plus water, to simulate soft clay behaviour considering the scale modeling. The undrained shear strength of the mix was measured using vane shear tests before and after placing the soft soil inside the laminar container which it can confirm the uniformity of the soil along the profile at several spots.

\subsection{Free Field Amplification}

\subsubsection{Cohesionless Soil}

Several analyses were used to explore the seismic response of different pile systems and the frequency content of earthquake motions measured on model piles and within the soil. The free field response of the liquefiable soil was deviated from applied input motion and experienced nearly similar values at two depth of base and surface in low-moderate shaking events. However,

free field motion for strong events such as K20 revealed rather comparably higher level of acceleration within the frequency range of $10-20 \mathrm{~Hz}$, followed by a de-amplification as a result of higher levels of nonlinearity and possibly liquefaction occurrence of the soil, ultimately loss of strength and sudden settlement. 


\subsubsection{Cohesive Soil}

Soft soil deposits are known to amplify shaking, though very strong shaking may attenuate surface motions due to soil nonlinearity and stiffness degradation. It is noted that the amplification factor clearly depends on soil stiffness. Free field acceleration response profile revealed significantly higher response acceleration within the soil profile compared to the input motion in relatively weaker events. As the intensity of input motion increased (e.g., K20), the level of amplification in the soil profile decreased. Correspondingly, lower amplification for soft soils exposed to strong shakings was reported which followed by the nonlinear response of soft clay under strong excitations as well as longer period of motion. Moreover, the higher peak amplitude of the ground acceleration and strain levels in the soil was related with higher material damping which additionally diminishes the response as the soil may have possibly suffered shear failure limiting the stress that can be transmitted to the surface soil.

\subsection{Soil-Pile-Structure Interaction (SPSI)}

As previously mentioned, hollow FRP piles and several varieties of conventional piles were comprehensively investigated in the past under load transfer tests in laboratory and medium-scaled condition in field. The purpose of this study was to complete a broad investigation on hollow FRP piles as a foundation alternative in supporting variety of structures and develop a collection of data to further analyse the seismic behaviour of specific types of hollow FRP piles (i.e., carbon and/or glass).

In the conducted shaking table tests in this thesis, soil-pile models were physically simulated, and the FRP pile group responses were compared based on the type (e.g., end-bearing or friction) and surrounding soil conditions. In both soil medium profiles (sand and soft clay), the peak accelerations and response spectra of FRP model piles were higher than those of the free 
field. This is attributed to strong interaction between the soil and the foundation. Correspondingly, the frequency content of the amplified motions appeared to be consistent for FRP model pile groups. Therefore, the soil-pile interaction has not deviated the frequency content of seismic motions recorded for both the conventional and FRP pile systems and only amplified the input motion.

\subsubsection{Sand}

According to the shaking table test results on end-bearing FRP piles, the divergence of amplification along the FRP piles were exactly at the same range of frequency content of the free field response. The shaking test results in liquefiable soils revealed that generation of pore water pressure has essentially affected the surface response of end-bearing FRP piles along with traditional piles since the rearrangement of sand particles due to gravity and tendency of contraction resulted in a sudden increase of pore water pressure, which it has ended with upsurge in density or sand densification. Therefore, this phenomenon has tolerated the surface response which carried larger kinematic forces as observed with increase in intensity of shaking. However, flexibility of end-bearing FRP piles, as it is a defined characteristic of FRP materials, was able to overcome this challenge and immersed the energy, as the FRP cap experienced lower response rate in comparison to its opponent, aluminium piles.

In contrast, further experimental tests were conducted to investigate the impact load transfer of FRP piles (i.e., frictional vs end-bearing) and the influence of SPI on the dynamic response of the model piles and their caps/structures. By comparing the experimental results obtained from the model piles, it can be concluded that all frictional model piles are fairly responded within similar range of amplification along the pile shaft, with marginally higher amplitude of response at the base of all aluminium model pile due to sophisticated material 
stiffness. Nevertheless, the major alteration was noted at the response of pile caps and its superstructure through kinematic soil-pile interaction and rocking motion. The aluminium pile cap experienced significantly higher acceleration and stronger rocking response compared to the FRP piles. Among the FRP piles, hollow GFRP pile demonstrated to achieve higher superiority to its competitor CFRP in liquefiable soil.

\subsubsection{Clay}

The acceleration response of free field motion beneath the cap/structure revealed slight attenuation which was primarily attributed to the strong nonlinearity within soft clay. The surrounding soil was evidently affected the response of FRP piles as the divergence of the pile behaviour throughout the shaft was attributed to the adhesion, pile material characteristics (e.g., flexural stiffness), and significant kinematic collaboration between the model piles and soft soil. The outcome of FRP response can be related to the inability of the model pile to abide by the wave motion due to the flexural rigidity of the piles. Lastly, CFRP piles have experienced higher motion response in base which in turn induced larger bending moment and hence, lower response closer to surface due to material flexibility. Alternatively, aluminium piles have reflected significant kinematic forces and seismic waves on the cap and structure which this result can also further underscore the importance of pile ductility and hence the kinematic pile-soil interaction on seismic response of pile foundations in seismic prone areas.

Additional shaking table tests were conducted to accomplish clearer understanding of the impacts that FRP model type (i.e., frictional) may have in overall performance of FRP model piles under seismic loading. It was noticed that frictional FRP and conventional model piles have equitably responded in similar fashion, as correspondingly the response was filtered near to the 
surface as larger response was carried out at the base. In general, CFRP have carried out the seismic loading with exceptional superiority compared to GFRP and aluminium model piles. This outcome was attributed to high flexibility FRP material and high strength-to-weight ratio which was able to distribute the seismic wave along the shaft and withstand smaller vibration at the cap. In comparison, aluminium pile tended to impose large bending moments close to surface indicating strong effect of SPI and deformation which caused soil-cap yielding and gapping. This has partially base-isolated the group by soil softening around the piles, and the aluminium pile cap response was ultimately more tolerated by rocking motion versus FRP piles.

\section{To conclude:}

The initiation to conduct these shaking tests were began by design of a 1-D laminar soil container and followed by idealized scale factor to provide specific site condition to investigate the response of hollow FRP group pile characteristics under seismic loading. The idea of performing these tests were initiated from previous studies which involved load transfer evaluation of hollow FRP piles (i.e., tensile and lateral testing in field) under static loading. The performance from past studies disclosed strong interaction of FRP pile material, high lateral capacity and larger material flexibility compared to traditional piles.

Based on the findings of this research, the seismic performance of FRP piles in both mediums of liquefiable soil and soft clay have remarkably placed these piles an option within the seismic performance range of conventional piles. In fact, result from pile deflection showed that the flexibility of FRP piles played a major role in reducing the loads experienced at the surface of pile and its cap. Meanwhile, these piles seem to be a feasible alternative for traditional piles especially in seismic prone area as well as in corrosive environment as they can be manufactured in variation of geometries, (i.e., in term of shape, diameter, wall thickness). However, there are a 
few inquiries which it needs further studies experimentally and numerically such as SPI effect within pile group size configurations. Based on the presented findings, FRP pile selection should be done depending on type of superstructure, , appropriate FRP material category, and site conditions. For instance, CFRP showed higher tensile strength, higher flexural stiffness (EI) (nearly similar to flexural stiffness of many conventional piles) and higher impact strength compared to GFRP. These advantages may place the CFRP as more viable pile to use in pilling industry.

\subsection{Recommendations}

The shaking table tests is by far one of the practical tools for generating physical data on the dynamic behaviour of soils and soil-pile interaction. The series of tests presented in this dissertation alone included six shaking events for a total of eight shaking table tests with maximum instrumentation capability in free field profile and pile-structure. While the purpose of this work was to assess performance of FRP piles and the influence of different parameters on the response of foundations in uniform mediums of sand or clay; experimental modification and numerical studies may develop new design procedures to consider the effect of the model pile type. Future research work may further assist to narrow down the study as expressed in the following statements:

- It should be noted that these tests were set to understand the behaviour of 2 by 2 FRP group piles, and further studies need be completed in order to understand the effect of pile arrangement and spacing in seismic response.

- Additionally, it is recommended to increase number of monitoring sensors on other piles within the same group to reach a better understanding on effects of FRP pile group interaction. 
- The interaction between cap/structure and soft soil is a poorly understood process, and continued research is needed to improve earthquake risk exposure for FRP piles which can include different types of structures in various site conditions. This can be completed using larger laminar boundary 2 by 2 meter to reduce the noise from instruments and models.

- Adopt the results of this study and similar works to develop a new design procedure. The proposed FRP pile design should be able to address the influence of pile type/material on the seismic response of pile cap and its superstructure in a simple but elaborative fashion.

- Perform further investigations on the cases where the cap is embedded, which are common case in practice, placing stress cells in the interface of soil and structure would support the soil-structure interaction under dynamic loading.

- Develop a physical shaking table model to consider the FRP piles with different characteristics such as driving impact, and pile group arrangement, similarly, the influence of soil layering in overall performance of FRP piles.

- Utilize numerical studies to determine seismic response of hollow FRP piles on various soil types under the influence of SPSI, and compare the findings of the current study which assessed over a broader range of seismic problems to further validate the FRP pile design. 


\section{References}

ASCE7-10. (2010). Minimum Design Loads for Buildings and Other Structures American Society of Civil Engineers.

ASTM D698-12e1. (2012) Standard Test Methods for Laboratory Compaction Characteristics of Soil Using Standard Effort (12 $\left.400 \mathrm{ft}-\mathrm{lbf} / \mathrm{ft}^{3}\left(600 \mathrm{kN}-\mathrm{m} / \mathrm{m}^{3}\right)\right)$, ASTM International, West Conshohocken, PA.

ASTM D854. (2014). Standard Test Methods for Specific Gravity of Soil Solids by Water Pycnometer." West Conshohocken, PA.

ASTM D2573. (2001). Standard Test Method for Field Vane Shear Test in Cohesive Soil. Annual Book of ASTM Standards, Vol. 04.08.

ASTM D-3966. (2011). Standard Test Methods for Deep Foundations Under Lateral Load, West Conshohocken, PA, USA.

ASTM. D4253. (2014). Standard test methods for maximum index density and unit weight of soils using a vibratory table. West Conshohocken, PA.

ASTM. D4254. (2014). Standard test methods for minimum index density and unit weight of soils and calculation of relative density. West Conshohocken, PA.

ASTM D-4945. (2008). Standard Test Method for High-Strain Dynamic Testing of Piles, ASTM International, West Conshohocken, PA, USA.

AS1170.4. (2007). Structural design actions-Earthquake actions in Australia. NSW, Australia: Standards Australia.

Anastasopoulos, I., Kourkoulis, R., Gelagoti, F. \& Papadopoulos, E. (2012). Rocking response of SDOF systems on shallow improved sand: An experimental study. Soil Dynamics and Earthquake Engineering, 40(0), 15-33.

Azizkandi A.S., Vahidinia S., Eslami M. (2020). Shaking table modeling of battered pile's behavior under seismic loading. In: Duc Long P., Dung N. (eds) Geotechnics for Sustainable Infrastructure Development. Lecture Notes in Civil Engineering, vol 62.

Balendra, T. and Heidebrecht, A.C. (1986). Influence of different sites on seismic base shear of buildings. Earthquake Engineering and Structural Dynamics, 14, 623-642.

Bao X., Morikawa Y., Kondo Y., Nakamura K., Zhang F. (2012). Shaking table test on reinforcement effect of partial ground improvement for group-pile foundation and its numerical simulation. Soils and Foundations. Vol. 52, Issue 6, Pages 1043-1061.

Bathurst, R. J., Keshavarz, A., Zarnani, S. \& Take, W. A. (2007). A simple displacement model for response analysis of EPS geofoam seismic buffers. Soil Dynamics and Earthquake Engineering, 27(4), 344-353. 
Blaney, G., and Mallow, W. (1987). Synthetic Clay Soil for Dynamic Model Pile Tests in Dynamic Response of Pile Foundations - Experiment, Analysis, and Observation, Geotech. Spec. Pub. 11, ASCE, 127-148.

Bogard, D. and Matlock, H. (1983). Procedures for the Analysis of Laterally Loaded Pile Groups in Soft Clay. Proc. Conf. Geotech. Practice in Offshore Eng., ASCE, Austin, 499-535.

Bonelli, P., Restrepo, J. I., Boroschek, R. \& Carvallo, J. F. (2012). The 2010 Great Chile Earthquake-Changes to Design Codes. International Symposium on Engineering Lessons Learned from the 2011 Great East Japan Earthquake, Tokyo, Japan.

Bowles, J. E. (2001). Foundation Analysis and Design. McGraw-Hill International. $5^{\text {th }}$ edn, Civil Engineering Series.

Bryden, P., El Naggar, H., and Valsangkar, A. (2015). Soil-Structure Interaction of Very Flexible Pipes: Centrifuge and Numerical Investigations. Int. J. Geomech., 15(6): 04014091.

Brown, D., Morrison, C., and Reese, L. (1988). Lateral Load Behavior of a Pile Group in Sand. J. Geotech. Eng., ASCE, 114(11), 1261-1276.

Brown, D. A., Reese, L. C., O'Neill, M. W. (1987). Cyclic Lateral Loading of a Large-Scale Pile Group. Journal of Geotechnical Engineering, 113(11), 1326-1343.

Brown, D., Shie, C., and Kumar, M. (1989). P-Y Curves for Laterally Loaded Piles Derived from Three-Dimensional Finite Element Model. Proc. 3rd Intl. Symposium on Numerical Models in Geomechanics, Niagara Falls, 683-690.

Building Seismic Safety Council, BSSC. (2001). NEHRP Recommended Provisions for Seismic Regulations for New Buildings, FEMA, Washington D.C., 2000 ed.

Busel, J. (1995). FRP Composites in Construction Applications, A Profile in Progress, SPI Composites Institute. New York, NY.

Capattia M. C., Dezib F., Carbonaria S., Gara D. (2018). Full-scale experimental assessment of the dynamic horizontal behavior of micropiles in alluvial silty soils. Soil Dynamics and Earthquake Engineering 113, 58-74.

Chau, K. T., Shen, C. Y. \& Guo, X. (2009). Nonlinear seismic soil-pile-structure interactions: Shaking table tests and FEM analyses. Soil Dynamics and Earthquake Engineering, 29(2), 300-310.

Chen, C. H., and Ueng, T. S. (2010). Behavior of Model Piles in a Liquefiable Soil in Shaking Table Tests. International Conferences on Recent Advances in Geotechnical Earthquake Engineering and Soil Dynamics. 2.

Chen Z., Wang, X., Zuo X., Du X., and Gao H. (2013). Shaking table test on the seismic failure characteristics of a subway station structure on liquefiable ground. Earthquake Engineering \& Structural Dynamics, vol. 42, no. 10, pp. 1489-1507.

Chiou J., Huang T.,Chen C., Chen C. (2021). Shaking table testing of two single piles of different stiffnesses subjected to liquefaction-induced lateral spreading. Engineering Geology. Volume $281,105956$. 
Cox, W. R., Reese, L. C., \& Grubbs, B. R. (1974). Field testing of laterally loaded piles in sand. Paper presented at the Offshore Technology Conference.

Daniel, I. M., and Ishai, O. (1994). Engineering Mechanics of Composite Materials. Ed. By Oxford University Press, New York.

Desai, C. S. \& Abel, J. F. (1972). Introduction to the finite Element Method: A Numerical Method for Engineering Analysis. Van Nostrand Reinhold Co., New York.

Dong Y., Feng Z., He J., Chen H., Jiang G., Yin H. (2019). Seismic Response of a Bridge Pile Foundation during a Shaking Table Test. Shock and Vibration, Article ID 9726013.

Dou, H. and Byrne, P. (1996). Dynamic Response of Single Piles and Soil-Pile Interaction. Canadian Geotech. J., 33(1), 80-96.

Durante M., Sarno L., Mylonakis G., Taylor C. and Lucio A. (2016). Simonelli1Soil-pilestructure interaction: experimental outcomes from shaking table tests. Earthquake Eng. Struct. Dynamic, 45:1041-1061.

Eid, W. K. (1987). Scaling Effect in Cone Penetration Testing in Sand. Ph.D. Thesis, Virginia Polytechnic Institute and State University, Virginia, USA.

Fam A. (2000). Concrete-filled fibre-reinforced polymer tubes for axial and flexural structural members. PhD thesis, University of Manitoba, Canada.

Fam, A. Z. and Miguel A. P. (2003). Precast piles for Route 40 bridge in Virginia using concrete filled FRP tubes. PCI Journal, vol. 48, (3), pp. 32-45.

Fam A., Boles R. and Robert M. (2016). Durability in a Salt Solution of Pultruded Composite Materials Used in Structural Sections for Bridge Deck Applications, Journal of Bridge Engineering, vol. 21, (1), pp. 4015032.

Finn W. D. (2005). A study of piles during earthquakes: Issues of design and analysis. Bulletin of Earthquake Engineering. Springer, 3, 141-234.

Finn, W. and Gohl, B. (1987). Centrifuge Model Studies of Piles Under Simulated Earthquake Lateral Loading in Dynamic Response of Pile Foundations - Experiment, Analysis, and Observation. Geotech. Spec. Pub. 11, ASCE, 21-38.

Gao X., Ling X., Tang L., Xu P. (2011). Soil-pile-bridge structure interaction in liquefying ground using shake table testing. Soil Dynamics and Earthquake Engineering. Vol. 31-7.

Gazetas G. (1982). Vibrational characteristics of soil deposits with variable wave velocity. International Journal for Numerical and Analytical Methods in Geomechanics, 6(1), 1-20.

Gazetas, G. and Mylonakis, G. (1998). Seismic Soil-Structure Interaction: New evidence andEmerging Issues. Proc. 3rd Conf. Geotechnical Earthquake Engineering and Soil Dynamics, ASCE, Seattle, 1119-1174.

Gazetas, G. (1991). Formulas and Charts for Impedances of Surface and Embedded Foundations. Journal of Geotechnical Engineering, 117(9), 1363-1381. 
Gazioglu, S. M., \& O'Neill, M. (1984). Evaluation of p-y relationships in cohesive soils. Analysis and design of pile foundations, 192-213.

Gerolymos, N. (1997). Effective Fundamental Period and Damping of Pile-Supported Bridge Piers. Diploma Thesis, National Technical University, Athens.

Gibson, G. (2010). Learning from Earthquakes, Chile, 2010. Australian Earthquake Engineering Society Conference (AEES). Perth, Western Australia: Australian Earthquake Engineering Society.

Giraldo, J., and Rayhani, M.T. (2014). Load transfer of hollow fiber-reinforced polymer (FRP) piles in soft clay. Transportation Geotechnics, 1(2014): 63-73.

Girault, D. P. (1986). Analyses of Foundation Failures. In: ASCE, ed. The Mexico Earthquakes-1985: Factors Involved and Lessons Learned. 178-192.

Gohl W. B. \& Finn, W. D. L. (1987). Seismic Response of Single Piles in Shaking Table Studies. Fifith Canadian Conference Earthquake Engineering. Ottawa. A.A. Balkema, 435444.

Goktepe, F., Celebi, E., \& Omid, A. J. (2019). Numerical and experimental study on scaled soilstructure model for small shaking table tests. Soil Dynamics and Earthquake Engineering, 119, 308-319.

Graziotti F., U. Tomassetti U., Kallioras S., Penna A., and Magenes G. (2017). Shaking table test on a full scale URM cavity wall building. Bulletin of Earthquake Engineering, vol. 15, no. 12 , pp. 5329- 5364.

Guades, E., Aravinthan, T., Islam, M. and Manalo, A. (2012). A review on the driving performance of FRP composite piles. Composite Structures, 94 pp. 932-1942.

Ha, I. S., Olson, S. M., Seo, M. W. \& Kim, M. M. (2011). Evaluation of reliquefaction resistance using shaking table tests. Soil Dynamics and Earthquake Engineering, 31(4), 682-691.

Haeri S. M., Kavand A., Rahmani I, Torabi H. (2012). Response of a group of piles to liquefactioninduced lateral spreading by large scale shake table testing, Soil Dynamics and Earthquake Engineering, Volume 38, Pages 25-45.

Hassini, S. and Woods, R. (1989). Dynamic Experiments with Model Pile Foundations. Proc. 12th Intl. Conf. Soil Mechanics Fdn. Eng., Rio de Janiero, Vol. 2, 1135-1138.

Helmi K., Fam A., Mufti A., and Hall J. M. (2006). Effects of driving forces and bending fatigue on structural performance of a novel concrete-filled fibre-reinforced-polymer tube flexural pile. Can. J. Civ. Eng. 33: 683-691.

Hetenyi, M. (1946). Beams on Elastic Foundation: Theory with Applications in the Fields of Civil and Mechanical Engineering, University of Michigan Press, Ann Arbor, Michigan.

Hokmabadi, A. S. (2014). Effect of Dynamic Soil-Pile-Structure Interaction on Seismic Response of Mid-Rise Moment Resisting Frames. PhD thesis, University of Technology Sydney (UTS), Australia. 
Hokmabadi A. S., Fatahi B., Samali B. (2014). Assessment of soil-pile-structure interaction influencing seismic response of mid-rise buildings sitting on floating pile foundations. Computers and Geotechnics. Vol. 55, Pages 172-186.

Hosseini M.A., Rayhani M. (2017). Evolution of pile shaft capacity over time in marine soils. International Journal of Geo-Engineering 8, 12.

Iai S. (1989). Similitude for shaking table tests on soil-structure-fluid model in $1 \mathrm{~g}$ gravitational field. Soilsand Found 29(1):105-118.

Idriss, I. (1990). Response of Soft Soil Sites During Earthquakes,” in H.B. Seed Memorial Symposium, Vol. 2, BiTech, 273-289.

Ishimura K., Ohtsuki A., Yokoyama K. \& Koyanagi Y. (1992). Sway rocking model for simulating nonlinear response of sandy deposit with structure. Tenth World Conference on Earthquake Engineering, 1897-1903.

Iskander, M. G., Hanna, S. and Stachula, A. (2001). Driveability of FRP Composite Piling. Journal of Geotechnical and Geoenvironmental Engineering, 127, pp. 169-176.

Iskander, M. G., Hassan, M. (1998). State of the Practice Review in FRP Composite Piling. Journal of Composites for Construction, 2(3), 116-120.

Iskander, M. G., Liu J., Sadek, S. (2002). Transparent Amorphous Silica to Model Clay. Journal of Geotechnical and Geoenvironmental Engineering, 128(3), 262-273.

Jakrapiyanun, W. (2002). Physical modeling of dynamics soil-foundation-structure- interaction using a laminar container. PhD thesis. University of California, San Diego.

Juran I., and Komornik U. (2006). Behavior of Fiber-Reinforced Polymer (FRP) composite piles under vertical loads. US Department of Transportation, Federal Highway Administration.

Kana, D., Boyce, L., and Blaney, G. (1986). Development of a Scale Model for the Dynamic Interaction of a Pile in Clay. J. of Energy Resources Technology, ASME, 108(3), 254-261.

Kanatani, M., Nishi, K. \& Touma, J. (1995). Large Shake Table Tests on Saturated Sand layer and Numerical Simulation by Nonlinear Analysis Mwethod. First International Conference on Earthquake Geotechnical Engineering, 1995 IS-Tokyo. 705-710.

Kim, Y. \& Roesset, J. (2004). Effect of Nonlinear Soil Behavior on Inelastic Seismic Response of a Structure. International Journal of Geomechanics, 4(2), 104-114.

Kim Y. J. (2019). State of the practice of FRP composites in highway bridges. Engineering Structures, Volume 179, 1-8.

Knappett, J.A., Haigh, S.K. \& Madabhushi, S.P.G. (2006). Mechanisms of failure for shallow foundations under earthquake loading. Soil Dynamics and Earthquake Engineering, Elsevier Science, 26(2-4): 91-102.

Kramer, S. L. (1996). Geotechnical earthquake engineering. Prentice Hall, Upper Saddle River, N.J. 
Kramer, S.L. and Stewart, J.P. (2004). Chapter 4: Geotechnical Aspects of Seismic Hazards, in Earthquake Engineering: From Engineering Seismology to Performance-Based Engineering, Y. Bozorgnia and V.V. Bertero (editors), CRC Press, 85 pages.

Kubo, K. (1969). Vibration Test of a Structure Supported by Pile Foundation. Proc. $4^{\text {th }}$ World Conf. Earthquake Eng., Santiago, A6:1-12.

Kwon, S. Y., Yoo, M. (2019). Evaluation of Dynamic Soil-Pile-Structure Interactive Behavior in Dry Sand by 3D Numerical Simulation. Applied Sciences, 9(13), 2612-.

Langhaar, H. (1951). Dimensional Analysis and Theory of Models, John Wiley and Sons, New York.

Lee, C. J., Wei, Y.C. \& Kuo, Y. C. (2012). Boundary effects of a laminar container in centrifuge shaking table tests. Soil Dynamics and Earthquake Engineering, 34(1), 37-51.

Li S., Zhou WH., Jing XY., Zhu HX. (2018) Evaluation of Grain Shape Parameters of Fujian Standard Sand. In: Wu W., Yu HS. (eds) Proceedings of China-Europe Conference on Geotechnical Engineering. Springer Series in Geomechanics and Geoengineering. Springer, Cham.

Lin L., and Wang L. (2006). Seismic slope behaviour in a large-scale shaking table model test. Engineering Geology, 86:118-133.

Ling X. Z., Gao X., Tang L., Su L. (2014). Effect of Shaking Intensity on Interactive Behaviour of Soil-Pile Group Foundations in Liquefiable Soil during Shaking Table Tests. International Efforts in Lifeline Earthquake Engineering.

Liu, H. and Chen, K. (1991). Test on Behavior of Pile Foundation in Liquefiable Soils. Proc. 2nd Intl. Conf. on Recent Advances in Geotech. Eng. and Soil Dyn., St. Louis, Vol. 1, $233-$ 235.

Liu W. G., Qin C., Liu Y. (2016). Shaking table tests on earthquake response characterization of a complex museum isolated structure in high intensity area. Shock and Vibration. Article ID 7974090.

Lu, X., Li, P., Chen, Y. \& Chen, B. (2004). Shaking Table Model Testing on Dynamic SoilStructure Interaction System. 13th World Conference on Earthquake Engineering, August 1-6, Vancouver, B.C., Canada.

Lu X., Zou Y., Lu W., and Zhao B. (2007). Shaking table model test on Shanghai world financial center tower. Earthquake Engineering \& Structural Dynamics. vol. 36, no. 4, pp. 439-457.

Luco, J.E., Trifunac, M.D., and Wong, H.L. (1988). Isolation of soil-structure interaction effects by full-scale forced vibration tests. J. Earthquake Engrg. Struct. Dynamics, 16, 1-21.

Ma S., \& Motazedian, D. (2012). Studies on the June 23, 2010 north Ottawa MW 5.2 earthquake and vicinity seismicity. Journal of Seismology, 16(3), 513-534.

Makris, N., Gazetas, G., and Delis, E. (1996). Dynamic Soil-Pile Foundation-Structure Interaction: Records and Predictions. Geotechnique, 46(1), 33-50. 
Martakis P., Taeseria D., and Chatzia E., Laue J. (2017). A centrifuge-based experimental verification of Soil-Structure Interaction effects. Soil Dynamics and Earthquake Engineering 103, 1-14.

Martinelli P. and Filippou F. C. (2009). Simulation of the shaking table test of a seven-story shear wall building. Earthquake Engineering \& Structural Dynamics, vol. 38, no. 5, pp. 587-607.

Mashhouda H. J., Yinb J., Panaha A., Leungb Y. (2018). Shaking table test study on dynamic behavior of micropiles in loose sand. Soil Dynamics and Earthquake Engineering 110, $53-$ 69.

Massimino, M. R. \& Maugeri, M. (2013). Physical modelling of shaking table tests on dynamic soil-foundation interaction and numerical and analytical simulation. Soil Dynamics and Earthquake Engineering, 49(0), 1-18.

Mason H.B., Trombetta N.W., Chen Z., Bray J.D., Hutchinson T.C., Kutter B. L. (2013). Seismic soil-foundation-structure interaction observed in geotechnical centrifuge experiments. Soil Dynamics and Earthquake Engineering 48, 162-174.

Matlock, H. (1962). Correlations for Design of Laterally Loaded Piles in Soft Clay. Rpt. To Shell Oil Co., Engineering Science Consultants, September.

Matlock, H. and Reese, L. (1960). Generalized Solutions for Laterally Loaded Piles. J. Soil Mechanics and Foundation Div., ASCE, 86(5), 63-91.

Maugeri, M., Musumeci, G., Novita, D. \& Taylor, C. A. (2000). Shaking table test of failure of a shallow foundation subjected to an eccentric load. Soil Dynamics and Earthquake Engineering, 20(5-8), 435-444.

Meymand, P. J. (1998). Shaking table scale model tests of nonlinear soil-pile-superstructure in soft clay. $\mathrm{PhD}$ thesis, University of California, Berkeley.

Mendoza, M.J. and Auvinet, G. (1988). The Mexico Earthquake of September 19,1985: behavior of building foundations in Mexico City. Earthquake Spectra Journal, EERI, Vol.4, No.4, pp.835 853 .

Mendoza, M. \& Romo, M. (1989). Behavior of Building Foundations in Mexico City During the 1985 Earthquake: Second Stage. Lessons Learned from the 1985 Mexico Earthquake. Earthquake Eng. Research Inst., 66-70.

Mindlin, R. D. (1936). Force at a Point in the Interior of a Semi-Infinite Solid. Physics, 7(5), 195-202.

Mirmiran A. and Shahawy M. (1996). A new concrete-filled hollow FRP composite column. Composites Part B: Engineering. Special Issue on Infrastructure. Elsevier Science Ltd. Vol. 27B. No. 34. pp. 263-268.

Mirmiran A., Shao Y., Shahawy M. (2002). Analysis and field tests on the performance of composite tubes under pile driving impact. Composite Structures, Volume 55, Issue 2. 
Mizuno, H. (1987). Pile Damage During Earthquake in Japan (1923-1983), in Dynamic Response of Pile Foundations - Experiment, Analysis, and Observation, Geotech. Spec. Pub. 11, ASCE, 53-78.

Mogami, T., and Kubo, K. (1953). The Behaviour of Soil During Vibration. Proceedings of the Third International Conference on Soil Mechanics and Foundation Engineering, Vol. 1, pp.152-153.

Mohamed H.M., Masmoudi R. (2010). Flexural strength and behavior of steel and FRP reinforced concrete-filled FRP tube beams. Engineering Structures, Volume 32, Issue 11,

Moncarz, P. \& Krawinkler, H. (1981). Theory and Application of Experimental Model Analysis in Earthquake Engineering. John Blume Earthquake Engineering Ctr., Stanford Univ.

Moss, R. E., Crosariol, V. \& Kuo, S. (2010). Shake Table Testing to Quantify Seismic Soil Structure Interaction of Underground Structures. International Conference on Recent Advances in Geotechnical Earthquake Engineering and Soil Dynamics, San Diego.

Motamed R., Towhata I., Honda T., Tabata K., Abe A. (2013). Pile group response to liquefactioninduced lateral spreading: E-Defense large shake table test, Soil Dynamics and Earthquake Engineering, Volume 51, pages 35-46.

Murugan, M., Muthukkumaran, K., \& Natarajan, C. (2017). FRP-strengthened RC piles. I: Piles under static lateral loads. Journal of Performance of Constructed Facilities, 31(3), 4017003. doi:10.1061/(ASCE)CF.1943-5509.0000990.

Nasr A. (2014). Experimental and theoretical studies of laterally loaded finned piles in sand. Canadian Geotechnical Journal. 51: 381-393.

$\mathrm{Ng}, \mathrm{K}$. W. (2011). Pile setup, dynamic construction control, and load and resistance factor design of vertically--loaded steel H-Piles. Graduate Theses and Dissertations. Paper 11924.

Nghiem, H. (2009). Soil-pile-structure interaction effects on high rises under seismic shaking. $\mathrm{PhD}$ Thesis, University of Colorado at Denver.

Nomura, S., Shamoto, Y., and Tokimatsu, K. (1991). Soil-Pile-Structure Interaction During Liquefaction. Proc. 2nd Intl. Conf. on Recent Advances in Geotech. Eng. And Soil Dyn., St. Louis, Vol. 1, 743-750.

Ohtomo, K. (1996). Effects of Liquefaction-Induced Lateral Flow on a Conduit with Supporting Piles, Proc. 11th World Conf. Earthquake Eng., Acapulco, Paper No. 386.

Pitilakis, D., Dietz, M., Wood, D. M., Clouteau, D. \& Modaressi, A. (2008). Numerical simulation of dynamic soil-structure interaction in shaking table testing. Soil Dynamics and Earthquake Engineering, 28(6), 453-467.

Poulos, H. G. (1971). Behaviour of laterally loaded piles: I-single piles and II- pile groups. Journal of Soil Mechanics and Foundation Divsion, ASCE, 97(SM5), 711- 731, 733751.

Prasad, S., Towhata, I., Chandradhara, G. and Nanjundaswamy, P. (2004). Shaking table tests in earthquake geotechnical engineering. Current science, 87(10), 13981404. 
Rayhani, M. (2007). Centrifuge modeling of seismic site response and soil structure interaction. $\mathrm{PhD}$ Thesis, The University of Western Ontario, Canada.

Reese, L., Cox, W., and Koop, F. (1975). Field Testing and Analysis of Laterally Loaded Piles in Stiff Clay. Proc. 7th Offshore Technology Conf., OTC 2312, Houston, Vol. 2, 671-690.

Rocha, M. (1957). The possibility of solving soil mechanics problems by the use of models. Proceedings of 4th International Conference of Soil Mechanics and Foundations Engineering, Vol.1 pp, 183-188.

Ross, G., Seed, H., and Migliacio, R. (1973). Performance of Highway Bridge Foundations in The Great Alaska Earthquake of 1964 - Engineering, Comm. on the Alaskan Earthquake of the Division of Earth Sciences, Natl. Research Council, Natl. Academy of Sciences, Wash., D.C.

Sakr, M., Naggar El, H. and Nehdi, M. (2007). Wave equation analyses of tapered FRP-concrete piles in dense sand. Soil Dynamics and Earthquake Engineering 27, 166-182.

Saranik M., Lenoir D., and Jézéquel L. (2012). Shaking table test and numerical damage behaviour analysis of a steel portal frame with bolted connections. Computers \& Structures, vol. 112113 , no. 4, pp. 327-341.

Sato, H., Tanaka, Y., Kanatani, M., Tamari, Y. \& Sugisawa, M. (1995). An Experimental and Numerical Study on the Behaviour of Improved Grounds. $1^{\text {st }}$ International Conference on Earthquake Geotechnical Engineering, IS-Tokyo. 767-772.

Scott, R., Tsai, C., Steussy, D., and Ting, J. (1982). Full-Scale Dynamic Lateral Pile Tests. Proc. 12th Offshore Technology Conf., OTC 4203, Houston, Vol. 1, 435-450.

Seed, H. and Clough, R. (1963). Earthquake Resistance of Sloping Core Dams. J. Soil Mechanics and Foundation Div., ASCE, 89(1), 209-242.

Seed, H. B., Ugas, C. and Lysmer, J. (1976). Site-dependent spectra for earthquake-resistance design. Bull. Seism. Soc. Am. 66, No.1, 221-243.

Seed, R., Dickenson, S., Riemer, M., Bray, J., Sitar, N., Mitchell, J., Idriss, I., Kayen, R., Kropp, A., Harder, L. Jr., and Power, M. (1990). Preliminary Report on the Principal Geotechnical Aspects of the October 17, 1989 Loma Prieta Earthquake. Rpt. No.UCB/EERC-90/05, Earthquake Eng. Research Ctr., Univ. of California.

Shao Y. (2003). Behavior of FRP - concrete beam - columns under cyclic loading. Raleigh, NC: North Carolina State University.

Srilatha N., Latha G. M., and Puttappa C. G. (2013). Effect of frequency on seismic response of reinforced soil slopes in shaking table tests. Geotextiles and Geomembranes, vol. 36, no. 1, pp. 27-32, 2013.

Stanton, J. F., Banerjee, S. \& Hasayen, I. (1998). Shaking table tests on piles. Final report, Research Project Y-2811, Task 26 Repared for Washington State Trasportation Communication. 
Stapleman. J. (1997). Pile on the Abuse. Composite Technology. Sept./Oct. 1997.

Steedman R. S., and Zeng, X. (1991). Physical Modelling of Earthquake Excitation for Geotechnical Engineering. Sixth Canadian Conference Earthquake Engineering.

Stewart, J.P., Fenves, J.L. and Seed, R.B. (1999). Seismic soil-structure interaction in buildings. I: Analytical methods. Journal of Geotechnical and Geo-environmental Engineering, Vol. 125, No. 1, pp: 26-37.

Stewart, J. P. \& Stewart, A. F. (1997). Analysis of Soil-Structure Interaction Effects on Building Response from Earthquake Strong Motion Recordings at 58 Sites. Rpt. No. UCB/EERC97/01. Earthquake Eng. Research Ctr., Univ. of California.

Sulaeman, A. (2010). The Use of Lightweight Concrete Piles for Deep Foundation on Soft Soils. $\mathrm{PhD}$ thesis in Civil Engineering, University of Tun Hussein Onn, Malaysia.

Taha, A.M. (2014). Static and Seismic Performance of Geosynthetics- Strengthened Pile Foundations. PhD thesis. The University of Western Ontario, Canada.

Tang, L. \& Ling, X. (2014). Response of a RC pile group in liquefiable soil: A shake-table investigation. Soil Dynamics and Earthquake Engineering. vol 67. 10.

Tang, L., Ling, X., Xu, P., Gao, X. \& Wang, D. (2009). Shake table test of soil pile groupsbridge structure interaction in liquefiable ground. Earthquake Engineering and Engineering Vibration, 1-12.

Tao, X., Kagawa, T., Minowa, C., and Abe, A. (1998). Verification of Dynamic Soil-Pile Interaction. Proc. 3rd Conf. Geotechnical Earthquake Engineering and Soil Dynamics, ASCE, Seattle, 1199-1210.

Taylor, C. A. (1997). Large Scale Shaking Tests of Geotechnical Structures. Earthquake Engineering Research Centre, University of Bristol.

Taylor, C. A., Dar, A. R. \& Crewe, A. J. (1995). Shaking table modelling of seismic geotechnical problems. 10th European Conference on Earthquake Engineers. Vienna, Austria. 441-446.

Tavenas, F., Roy, M., and La Rochelle, P. (1973). An Artificial Material for Simulating Champlain Clays, Can. Geotech. J., 10(3), 489-503.

Timoshenko, S. (1940). Strength of materials ( $\left.2^{\text {nd }} e d\right)$. D. Van Nostrand Company. Inc. New York.

Torabi, H. (2016) A Hybrid Approach for Nonlinear Soil-Structure Interaction Analysis of PileSupported Bridges. PhD Thesis. Carleton Universiy, Ottawa. Canada.

Tsukamoto, Y., Ishihara, K., Sawada, S. \& Fujiwara, S. (2012). Settlement of Rigid Circular Foundations during Seismic Shaking in Shaking Table Tests. International Journal of Geomechanics, 12(4), 462-470.

Turan, A. (2009). Physical modeling of seismic soil-structure interaction of embedded structures. $\mathrm{Ph} . \mathrm{D}$. Thesis, The University of Western Ontario, Canada.

Turan A, El Naggar H. (2008). Lateral behavior of micropile groups under static and dynamic loads. in: Proceedings of 4th Canadian conference on geohazards. 
Ueng, T. S., Wang M. H., Chen, M. H., Chen C. H., and Peng, L. H. (2006). A large biaxial shear box for shaking table test on saturated sand. Geotechnical Testing Journal, 29(1).

Unsever Y. S., Matsumoto T., Esashi K., Kobayashi S. (2017). Behaviour of model pile foundations under dynamic loads in saturated sand. Bull Earthquake Eng., 15:1355-1373.

Valsangkar, A. J., Dawe, J. L. \& Mita, K. A. (1991). Shake Table Studies of Seismic Response of Single Partially Supported Piles. Sixth Canadian Conference Earthquake Engineering. 327-334.

Veletsos, A. S., and Meek, J. W. (1974). Dynamic behaviour of building-foundation systems. Earthq. Engrg. and Struct. Dyn. 3, 121-138.

Veletsos, A. \& Prasad, A. (1989). Seismic Interaction of Structures and Soils: Stochastic Approach. Journal of Structural Engineering, 115(4), 935-956.

Wang, S., Kutter, B., Chacko, J., Wilson, D., Boulanger, R., and Abghari, A. (1998). Nonlinear Seismic Soil-Pile-Structure Interaction, Earthquake Spectra, 14(2), 377-396.

Wilson D.W. (1998). Soil-pile-superstructure interaction in liquefying sand and soft clay. $\mathrm{PhD}$ Thesis. University of California, Davis, USA.

Wolf, J. P. (1985). Dynamic soil-structure interaction. Wolf, Prentice-Hall, Englewood Cliffs, New Jersey.

Wood, H. (1908). Distribution of Apparent Intensity in San Francisco in the California Earthquake of April 18, 1906. Rpt. of the State Earthquake Investigation Comm.,Carnegie Inst. of Washington, Washington, D.C., 220-245.

Wu Q., Ding X., Chen Z., Zhang Y. (2020) Shaking Table Tests on Seismic Responses of Pilesoil-superstructure in Coral Sand, Journal of Earthquake Engineering.

Yang, Z. J., Zhang, X. R., Yang, R., Zhou, X., \& Niu, F. (2018). Shake table modeling of pile foundation performance in laterally spreading frozen ground crust overlying liquefiable soils. Journal of Cold Regions Engineering, 32(4).

Yang, J., Lu, Z., \& Li, P. (2020). Large-scale shaking table test on tall buildings with viscous dampers considering pile-soil-structure interaction. Engineering Structures, 220, 110960.

Yao, S. (1980). Dynamic and Static Test of Model Piles or Pile Groups. Proc. $7^{\text {th }}$ World Conf. Earthquake Eng., Istanbul, Vol. 3, 459-466.

Yasuda S., Ishihara K., Morimoto H, Iwao M., Rolando O., Ikeda M., and Tamura S. (2000). Large-scale shaking table tests on pile foundations in liquefied ground. IITK.

Zaghi A. E., Saiidi M. S., Mirmiran A. (2012). Shake table response and analysis of a concrete filled FRP tube bridge column, 94(5), 1564-1574.

Zen, K., Yamazaki, H., Toriihara, M. \& Mori, T. (1992). Shaking Table Test on Liquefaction of Artificially Cemented Sands. Tenth World conference on Earthquake Engineering, Madrid, Spain. 1417-1420. 


\section{Appendices}

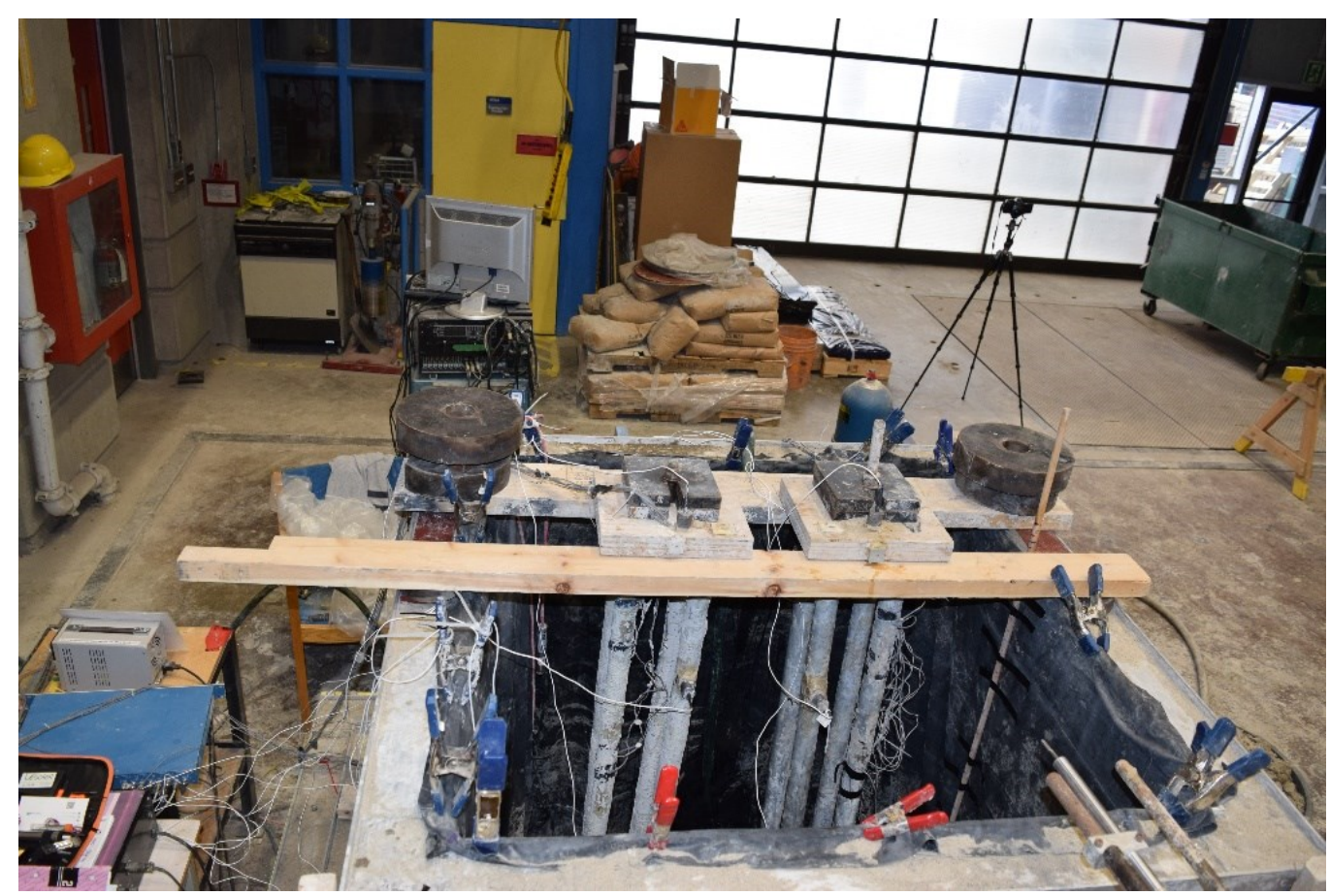

Frictional GFRP and aluminium group piles prepared followed by sand pluviation

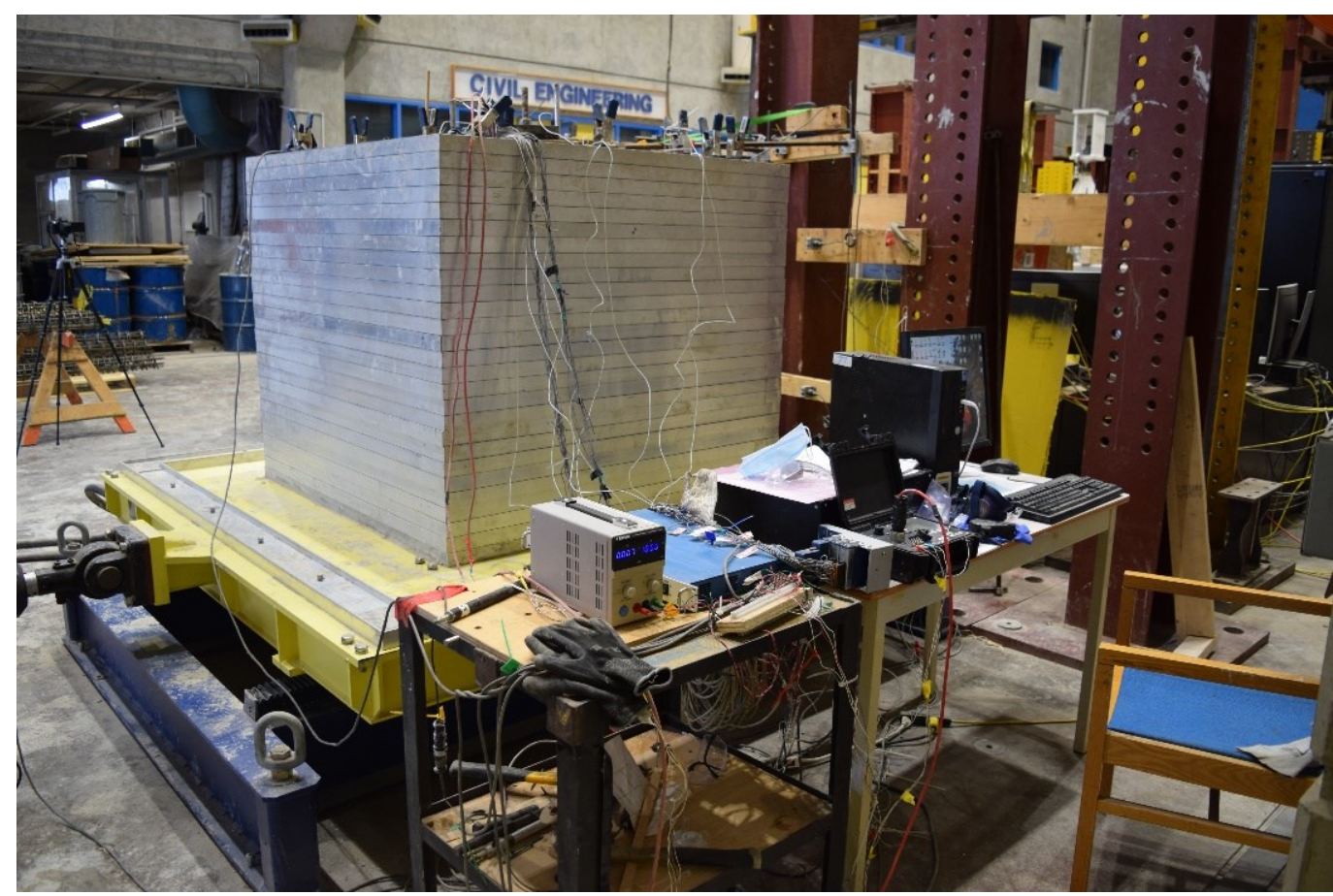

Shaking Table setup 


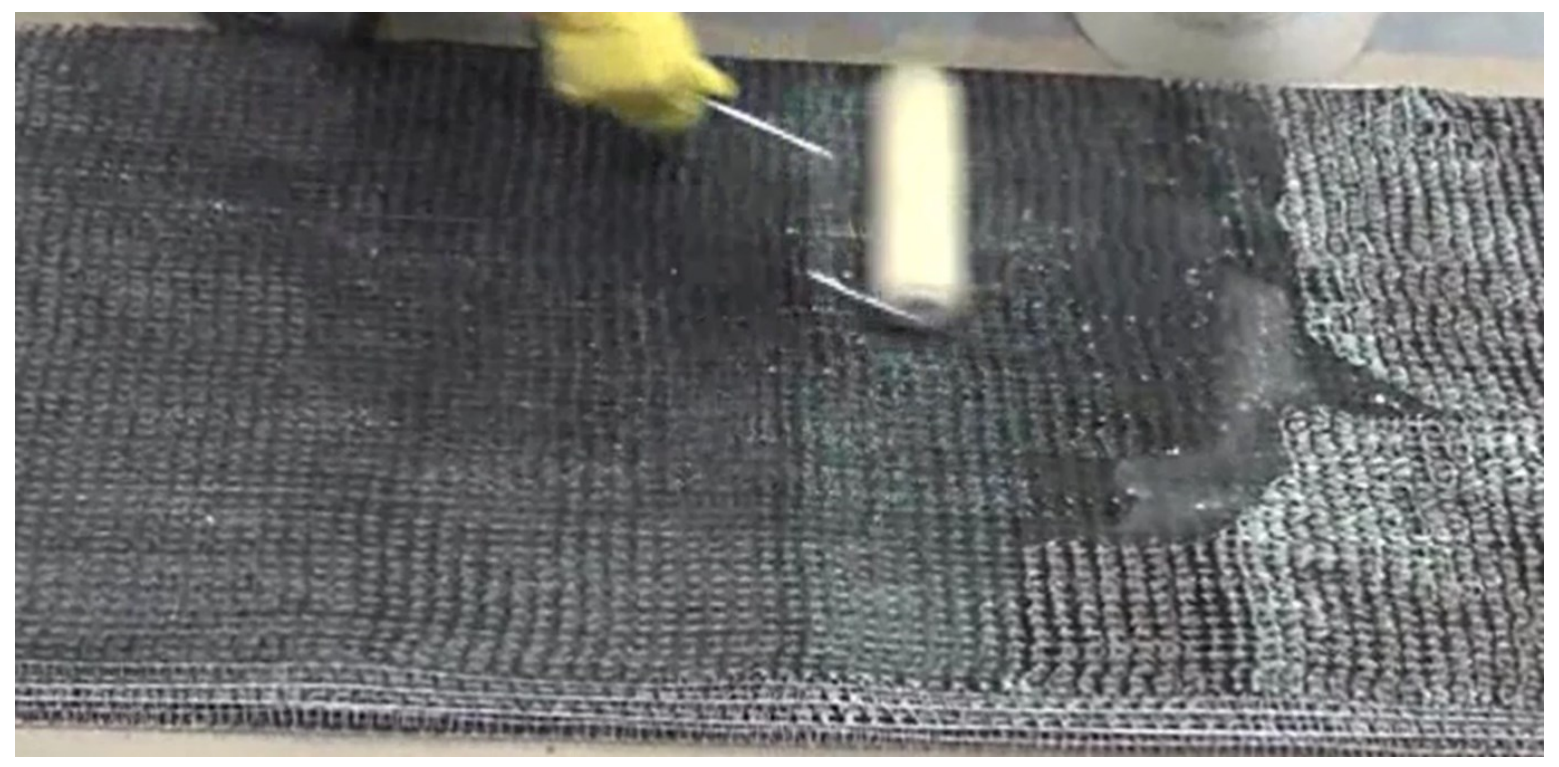

Saturated CFRP

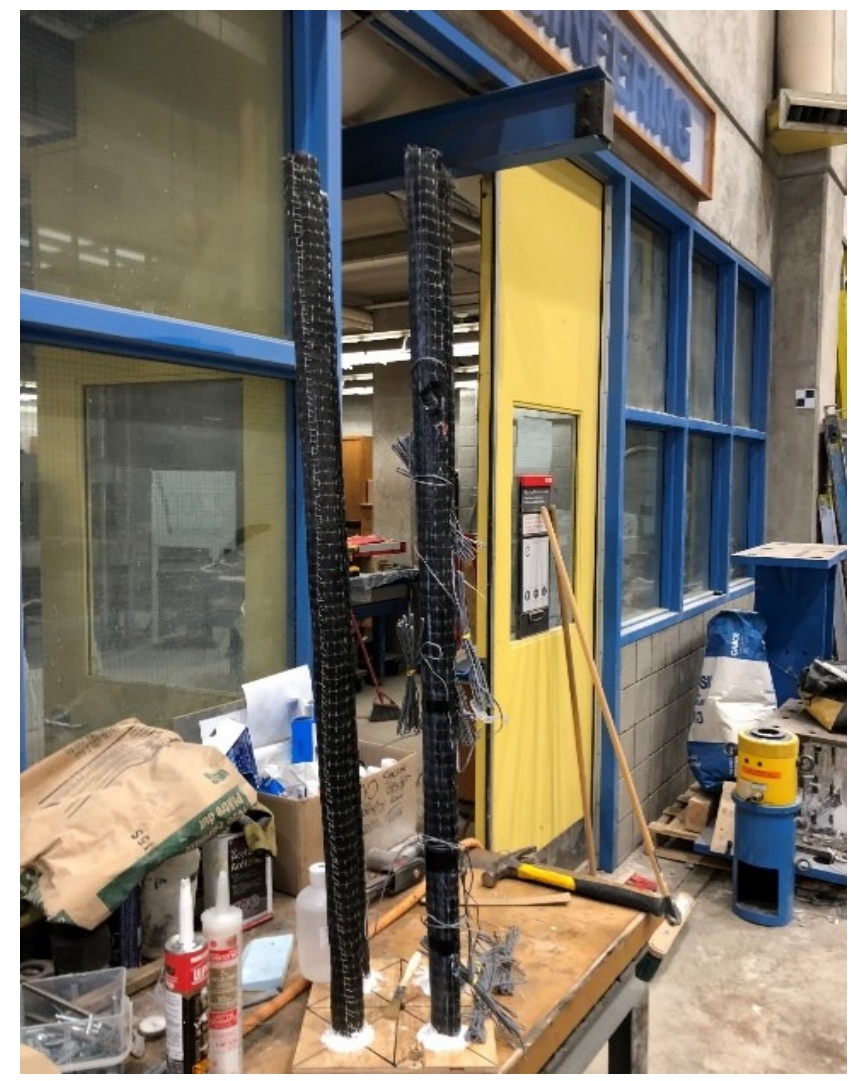

CFRP model pile-prepared for instrumentation 


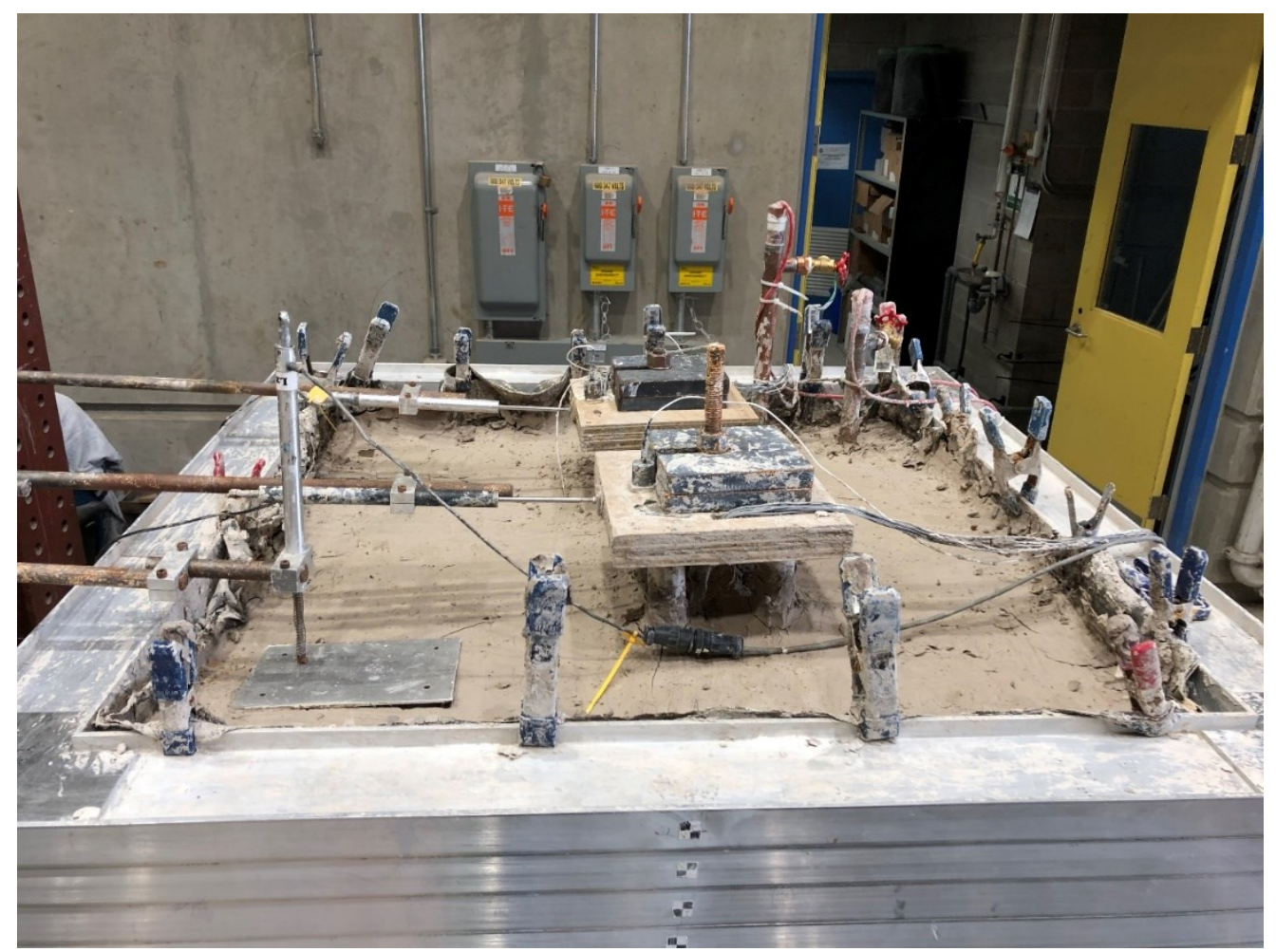

Prepared laminar container for model piles embedded in soft clay

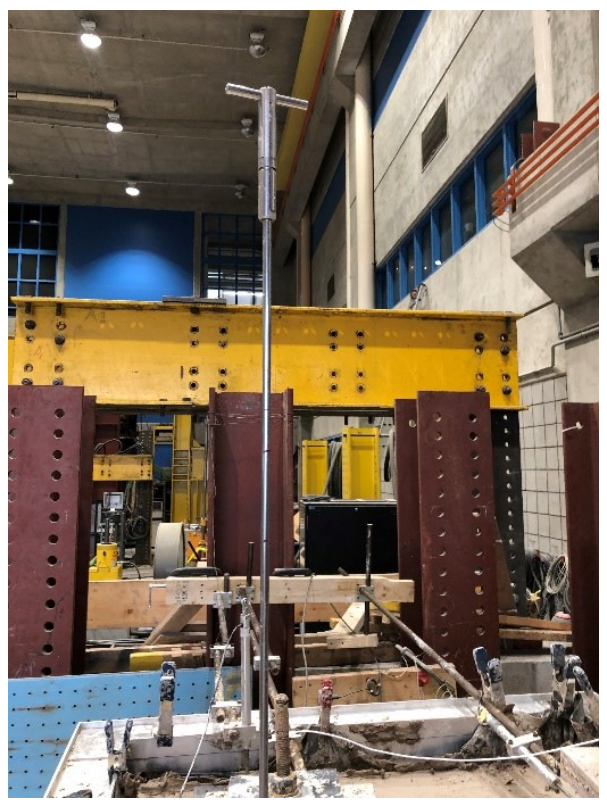

Vane Shear Test before and after each test (ASTM D2573) 




Occurance of full liquefaction upon completion of last shaking event (i.e., K20) 